\title{
PERFORMANCE ANALYSIS AND LIFE PREDICTION FOR SMALL WIND TURBINE BLADES: A WOOD LAMINATE CASE STUDY
}

\author{
A Thesis \\ presented to \\ the Faculty of the College of Engineering \\ California Polytechnic State University \\ In Partial Fulfillment \\ of the Requirements for the Degree \\ Master of Science in Mechanical Engineering
}

By

Christopher James Nosti

August 2009 


\section{COMMITTEE MEMBERSHIP}

TITLE:

Performance Analysis and Life Prediction for Small Wind Turbine Blades: A Wood Laminate Case Study

AUTHOR: $\quad$ Christopher James Nosti

DATE SUBMITTED: $\quad$ August 2009

COMMITTEE CHAIR: $\quad$ Patrick Lemieux, Ph.D.

COMmitTeE MEMBER: Joseph Mello, Ph.D.

COMMITTEE MEMBER: John Ridgely, Ph.D. 


\author{
ABSTRACT \\ Performance Analysis and Life Prediction for Small Wind Turbine Blades: A Wood \\ Laminate Case Study \\ Christopher James Nosti
}

A detailed study of the fatigue life of wooden wind turbine blades for a new 10 kilowatt system was undertaken. A numerical model of the blades was created using the technical software package MATLAB in order to estimate the maximum stress occurring within the blade in response to changes in wind velocities based on a wind profile approximating the location where these turbines are expected to operate. The material properties of the wooden laminate were measured using an Instron tensile test machine and were found to be in line with published values. In parallel with this effort, a three dimensional part scanner was utilized to compare the as-built blade to the theoretical profile and large differences between these two profiles were observed. These studies were then used to guide the creation of a full scale fatigue test which subjected two blades to accelerated fatigue loads in order to monitor the damage accumulation within each blade. It was ultimately determined that this new blade design has a fatigue life greater than 20 years.

Keywords: wind, turbine, blade, fatigue, composite, wood, laminate, performance, fullscale, testing. 


\section{ACKNOWLEDGMENTS}

This study was performed at California Polytechnic State University, San Luis Obispo under the primary guidance of Professor Patrick Lemieux, and with contributions from Professor Joseph Mello, Professor John Ridgely, Professor Hal Gascoigne and Jim Gerhardt. I would like to thank each of them greatly for their insight, guidance and support. I would also like to thank my fellow graduate students, David Ulrich, Estevan Negrete, Matt Scott, Stephane Roussel, George Katsanis, Devin Gosal and Brian Edwards for their support and camaraderie throughout the project. I would also like to thank my parents Gary and Robin and my sisters Ali and Jackie for their love and support throughout this project. And most importantly, I would like to thank my girlfriend Christy, without her unwavering love and support I would not have been able to complete this project. 


\section{TABLE OF CONTENTS}

List of Figures

Chapter 1. Introduction and Overview ............................................................................. 1

Chapter 2. Small Wind Turbine Systems, Current Practice and Testing Methods ...................... 6

2.1 SMALL WIND TURBINE SYSTEMS ………………………..................................................... 6

2.1.1 Typical Small Wind Turbine Components ............................................................................ 6

2.1.2 Types of Loading on the Blades of a Small Wind Turbine ..............................................

2.1.3 Sources of Loading on the Blades of a Small Wind Turbine .......................................... 9

2.1.3.1 Gravitational Loading ......................................................................................................

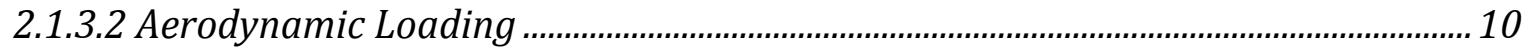

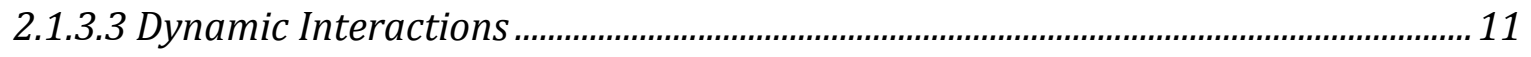

2.2 THE 10KW GORMAN CONTROLS WIND TURBINE ............................................................. 13

2.2.1 Basic Design of the System ........................................................................................... 13

2.2.2 Manufacturing the Blades for the Gorman Controls' Wind Turbine ......................... 15

2.3 SURVEY OF CURRENT SMALL WIND TURBINE BLADE TESTING METHODS ................. 17

2.3.1 In-Field Blade Root Strain Measurement..................................................................... 17

2.3.2 Blade Fatigue Life Testing............................................................................................ 19

Chapter 3. Analytical Methods for Determining Fatigue Life................................................ 22

3.1 FATIGUE OF WOODEN STRUCTURES.............................................................................. 22

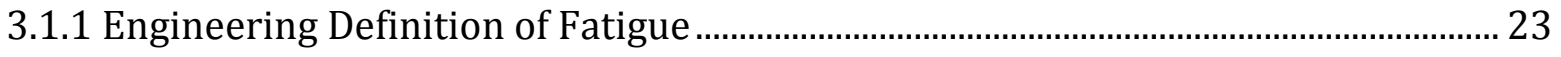

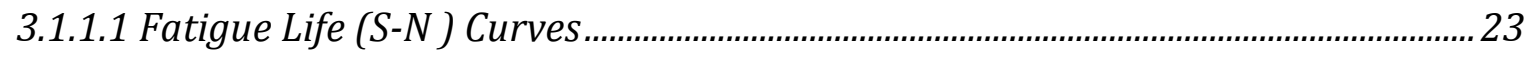

3.1.1.2 Alternating Stresses.................................................................................................24

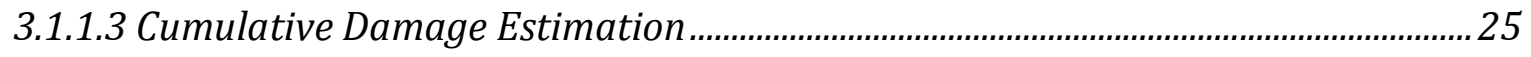


3.1.2 Fatigue Properties of Wood...................................................................................... 26

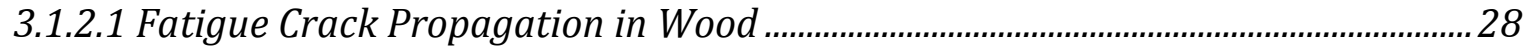

3.1.3 Derivation of S-N Master Curves for Wooden Laminate............................................... 29

3.2 CREATION OF ANALYTICAL WIND LOADING SPECTRUM.................................................. 31

3.2.1 Rainflow Cycle Counting Method ………………......................................................... 32

3.2.2 Rainflow Cycle Counting the Wind Spectrum ............................................................... 33

3.2.2.1 MATLAB Rainflow Cycle Counting Program................................................................ 33

3.2.2.2 Results of Rainflow Cycle Counting................................................................................. 34

3.2.3 The Weibull Distribution................................................................................................. 35

3.2.4 Weibull Parameter Estimation........................................................................................ 36

3.3 NUMERICAL BLADE RESPONSE MODEL.............................................................................. 38

3.3.1 Blade Element Momentum Theory............................................................................... 39

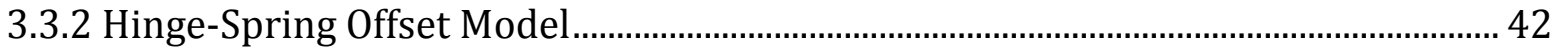

3.3.3 Implementation of MATLAB Blade Model ....................................................................... 43

3.3.4 Numerical Blade Response Model Results .................................................................... 44

3.4 EQUIVALENT DAMAGE FATIGUE LIFE ESTIMATION ………............................................. 46

3.4.1 Stress Cycle Estimation ................................................................................................... 46

3.4.2 Cumulative Fatigue Damage Totals ........................................................................... 50

Chapter 4. Experimental Equipment, Methods and Results .................................................. 51

4.1 IN-FIELD BLADE ROOT STRAIN MEASUREMENT ………………………………………... 51

4.1.1 Wireless Data Acquisition Device …………………………………………………... 52

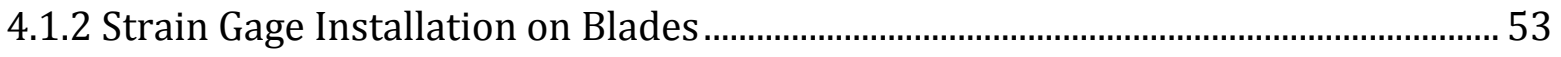

4.1.3 Attempted In-Field Blade Load Measurement............................................................. 54

4.2 MATERIAL PROPERTY TESTING ....................................................................................... 56 


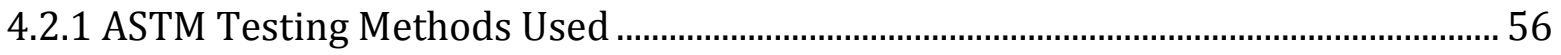

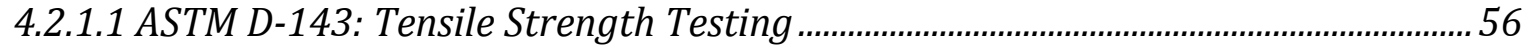

4.2.1.2 ASTM D-143: Compressive Strength Testing .............................................................. 57

4.2.2 Wood Coupon Sizing ……………………………………………………………….. 57

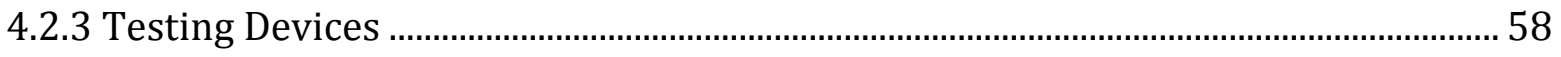

4.2.4 Material Property Estimation...................................................................................... 59

4.2.4.1 Stress-Strain Relationships for Transversely Isotropic Materials............................ 61

4.3 THREE DIMENSIONAL BLADE PROFILE VERIFICATION STUDY ........................................ 63

4.3.1 Blade Scanning Procedure ............................................................................................... 63

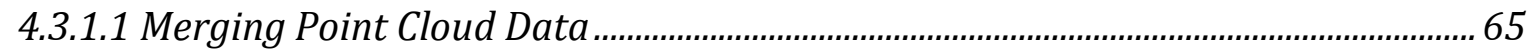

4.3.2 Blade Profile Comparisons and 3D Model Verification ................................................ 67

4.3.2.1 Comparison with Clark Y Airfoil ..................................................................................... 68

4.3.2.2 Section Profile Variation ….............................................................................................. 70

4.4 FULL SCALE BLADE TESTS............................................................................................... 71

4.4.1 Mechanical Test Fixture Components ............................................................................... 72

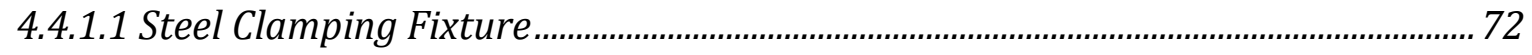

4.4.1.2 Support Fame

4.4.1.3 Driving Crank and Motor ...................................................................................... 75

4.4.1.4 Wooden Blade Protection Barrier ................................................................................... 76

4.4.2 Electronic Equipment and Circuits................................................................................. 78

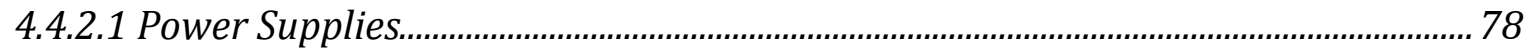

4.4.2.2 Motor Power Supply Circuit..........................................................................................

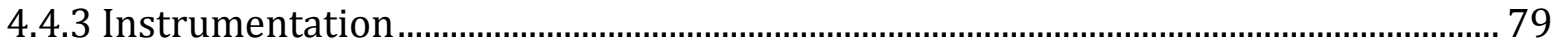

4.4.3.1 Blade Tip Deflection Measurement................................................................................. 79

4.4.3.2 Cumulative Cycle Count Monitoring............................................................................. 80 
4.4.3.2.1 Hall-Effect Cycle Counter............................................................................... 81

4.4.3.2.2 Bicycle Computer Cycle Counter ……………………………………………..... 81

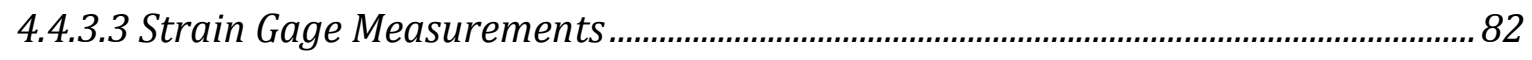

4.4.3.4 Strain Gage Placements......................................................................................... 83

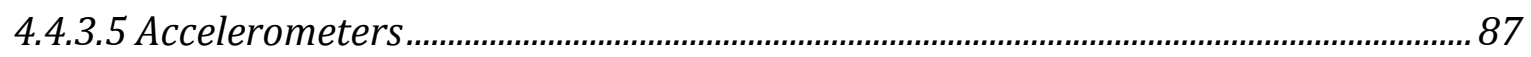

4.4.4 Testing Regime ......................................................................................................... 88

4.4.4.1 Static Loading Tests............................................................................................. 88

4.4.4.2 Frequency Response Tests ............................................................................................ 90

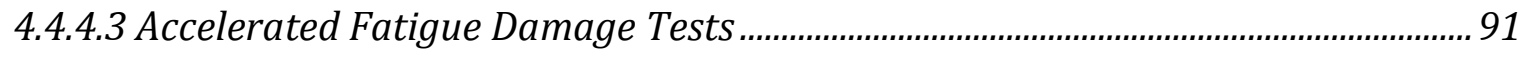

4.4.5 Data Acquisition Program and Methods............................................................................ 93

4.4.5.1 Data Storage Channels...................................................................................................94

4.4.6 Data Post Processing .............................................................................................. 95

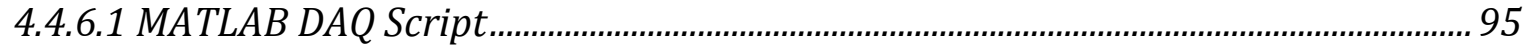

4.5 FULL SCALE FATIGUE LIFE TESTING RESULTS ………………………………………...... 97

4.5.1 Individual DAQ Channel Results.................................................................................... 97

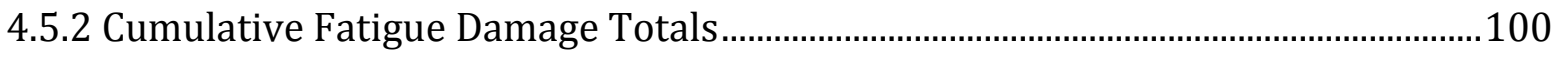

4.5.3 Blade Response Degradation ...................................................................................... 102

Chapter 5. Conclusions and Recommendations ................................................................ 106

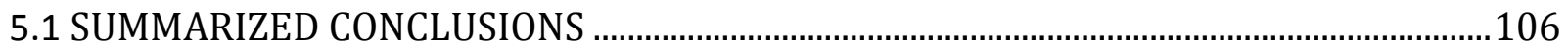

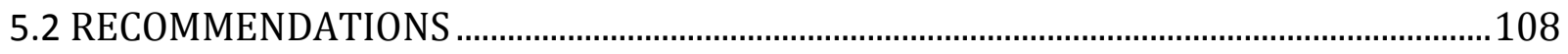

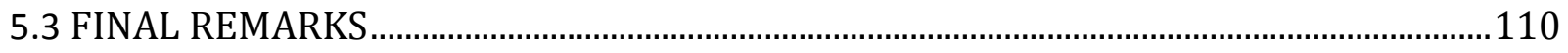

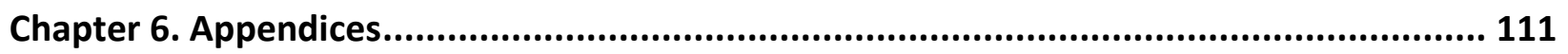

Appendix A. Derivation of Equations ......................................................................... 111 
DERIVATION OF AERODYNAMIC EQUATIONS ………………................................................111

BLADE ELEMENT MOMENTUM THEORY ………………….....................................................115

HINGE SPRING OFFSET MODEL EQUATION DERIVATIONS ..................................................118

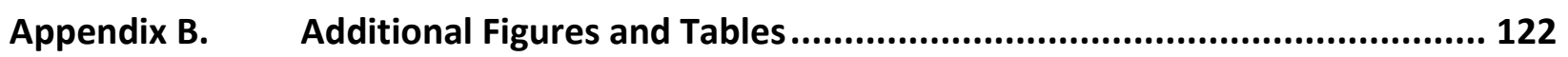

CHAPTER 3. FIGURES AND TABLES......................................................................................122

CHAPTER 4 FIGURES AND TABLES.........................................................................................129

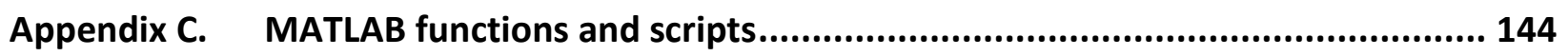

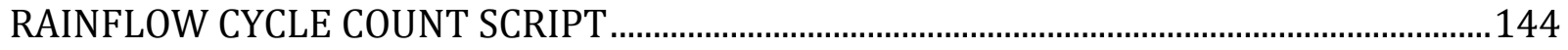

RAINFLOW CYCLE COUNT HISTOGRAM FUNCTION ............................................................145

RAINFLOW CYCLE COUNT MATRIX FUNCTION ......................................................................146

NUMERICAL BLADE RESPONSE MODEL .............................................................................149

MYKELSTAD METHOD/ROTATING BEAM NATURAL FREQUENCY CALCULATOR ..........162

DATA ACQUISITION MATLAB SCRIPT …………………........................................................166

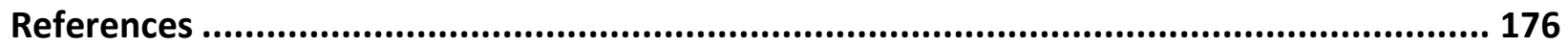




\section{LIST OF FIGURES}

\section{Chapter 2. Small Wind Turbine Systems, Current Practice and Testing Methods}

Figure 2-1. Typical components of a small wind turbine system: 1) Blades, 2) Hub, 3)

Gearbox/Drivetrain, 4) Generator, 5) Nacelle, 6) Tail Vane, 7) Yaw Bearing, 8) Tower........................................ 7

Figure 2-2. The loading on wind turbine blades due to gravitational and inertial forces...........................................10

Figure 2-3. Induced aerodynamic forces due to a relative wind velocity, $U_{\text {rel. }} . F_{L}$ is the airfoil lift force, $F_{D}$ is the airfoil drag force, $r$ is the radius of rotation measured from the hub center, $\omega$ is the angular velocity of the rotor, $\alpha$ is the angle of attack, $\Phi$ is the angle of the relative wind, $\theta_{p}$ is the section pitch angle, $a$ is the axial induction factor and $\mathrm{a}^{\prime}$ is the angular induction factor.

Figure 2-4. Side view of a wind turbine showing the fictitious centrifugal force and the derived components in the radial and normal directions (Hansen and Martin 2008).

Figure 2-5. The axial flux generator for the $10 \mathrm{~kW}$ Gorman Controls' wind turbine mounted on a testing rig during a stator coil test.

Figure 2-6. The Gorman Controls' 10 kilowatt wind turbine shown with the tail vane in the raised or furled position. . .15

Figure 2-7. A top view of the Gorman Controls' wind turbine demonstrating the offset between the tail vane, the axis of rotation of the blades and the tower axis. $U_{o}$ is the relative wind velocity, $M_{\text {rotor }}$ is the moment induced by the rotor and $\mathrm{M}_{\text {tail }}$ is the moment induced by the tail vane. . .15

Figure 2-8. A finished wooden laminate blade for the Gorman Controls' $10 \mathrm{~kW}$ wind turbine. .. .17

\section{Chapter 3: Analytical Methods for Determining Fatigue Life}

Figure 3-1. Illustration of non-zero mean alternating stress cycles

Figure 3-2. Illustration of the principal directions relative to the fiber direction in wood. 1) longitudinal direction, 2) radial direction, 3) tangential direction. . .27

Figure 3-3. Derived S-N master curve for eastern white pine laminate at stress ratio $\mathrm{R}=-1$................................31

Figure 3-4. Illustration of rainflow cycle counting method of measuring half cycles. .................................................33

Figure 3-5. Preliminary results of rainflow cycle counting the WEICAN 2004 year $30 \mathrm{~m}$ tower data. . .35

Figure 3-6. Linear form of the Weibull probability density function used to estimate the Weibull scale (a) and shape (b) factors for the WEICAN wind spectrum. . .37

Figure 3-7 Weibull probability function with relative frequency density overlay showing curve fit for WEICAN wind data. .38

Figure 3-8. Linear approximation of a wind turbine as an actuator disk and stream tube where $U$ is the mean air speed at the four locations shown. (Manwell, McGowan and Rogers 2002) 
Figure 3-9. Numerically predicted blade root flapwise bending stress as a function of tip deflection out of the plane of rotation.

Figure 3-10. Numerically predicted normalized blade root flapwise bending stress as a function of wind speed. 45

Figure 3-11. Weibull curve fit for stress cycles from binned data at $\mathrm{R}=0.1$. .49

\section{Chapter 4: Experimental Equipment, Methods and Results}

Figure 4-1. Wireless Data Acquisition Device mounted on PVC support frame and rotor center support..........52

Figure 4-2. Sample output during a bench natural frequency test using the wireless data acquisition device.

Figure 4-3. Dual strain gages attached to the root of the wind turbine blade with lead wires for Wheatstone bridge attachment.

Figure 4-4. Wireless DAQ attached to the rotor center with two Wheatstone strain gage sensing circuits attached to the blade root. .55

Figure 4-5. Gorman Controls' 10kW wind turbine immediately after falling from the 30 meter tower. .55

Figure 4-6. Illustration of location within blade root where tensile and compressive wood coupons were

cut. 1) is the wood grain direction and 2) is the perpendicular grain direction. . .58

Figure 4-7. a) The Instron 4400R tensile test machine used to determine the Young's Modulus of the wood. b) The location of strain gages on a sample tensile test coupon.

Figure 4-8. Sample test performed to estimate the modulus of elasticity in the longitudinal or fiber direction. Stress is shown as a function of strain. .60

Figure 4-9. The LDI PS 4000 laser part scanner shown during a scan of blade C. The associated computer workstation can be seen in the background.

Figure 4-10. Blade B shown during the scanning process with white beads glued to the surface as reference points.

Figure 4-11. Illustration of the surface and hole repair operations performed when merging the many point cloud data files for each blade. a) Holes before repair, b) repaired surface.

Figure 4-12. The completed 3D scanned model of blade A, as viewed from within the CAD software package SolidWorks.

Figure 4-13. Six 2D profiles generated by sectioning the 3D model of blade B.

Figure 4-14. Example of the profile matching process for profile of blade number $\mathrm{A}$ at a radial location of 90 inches with a chord length of 8.76 inches. Clark Y profile is shown in green and the as-built profile is shown in red.

Figure 4-15. The vibration test clamping fixture as seen within SolidWorks. The blade attaches between the flat green plates. 
Figure 4-16. Support frame and blade vibration fixture as designed within SolidWorks (a) and the as-

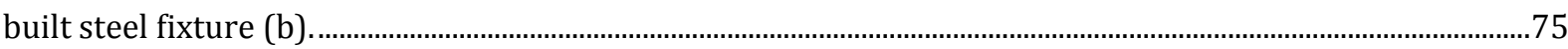

Figure 4-17. Crank disk and connecting rod used to drive the blade vibration fixture. .77

Figure 4-18 Full scale wooden blade fatigue life testing fixture showing wooden barrier constructed to protect the blade during testing. . .77

Figure 4-19. Motor power supply circuit showing on/off switch and fault switch. . .79

Figure 4-20. Picture of the blade fault switch attached to plywood barrier.. . .79

Figure 4-21. Blade tip deflection device showing vertical measurement graduations, wooden clamps and rubber band height indicators.

Figure 4-22. Orientation of the bicycle and Hall-Effect cycle count sensors. Magnets on the tip of the blade may be seen, a) is the Hall-Effect sensor and b) is bicycle computer sensor. . .82

Figure 4-23. Illustration of strain gage locations for both uniaxial, (a, c, e) and rectangular rosettes (b, d) on the root of the blade. . .85

Figure 4-24. Strain gage connections as used during the accelerated fatigue life testing of blade A................. . .86

Figure 4-25. Example of strain gage output during accelerated fatigue life test number four for blade A.........86 Figure 4-26. Accelerometers used during testing as mounted on the driving input arm, (a), and blade tip,

(b).

Figure 4-27. Comparison of the statically measured and predicted blade root bending stress as a function of tip displacement. . .90

Figure 4-28. Example of tap-test response and MATLAB data analysis to find system natural frequency after duration test six for blade B.

Figure 4-29. Validation of the assumption that the accelerated fatigue life tests subjected each blade to an alternating stress level equal to $\mathrm{R}=-1$. The strain level is seen to alternate from positive and negative 200 micro strain.

Figure 4-30. Plot of all strain gage letter A data for the entire testing life of blade A normalized by tip deflection distance. Note the loss of data after test six.

Figure 4-31. Strain gage signal offset observed between individual tests for strain gage pair A during the accelerated testing of blade $\mathrm{A}$.

Figure 4-32. Illustration of total amount of fatigue damage accumulation during each sequential test for blade $\mathrm{A}$ and blade $\mathrm{B}$. 101

Figure 4-33. Cumulative damage as a function of total number of testing cycles for blade A and blade B.......101

Figure 4-34. Observed decrease in effective stiffness and natural frequency during accelerated fatigue

life test of Blade $\mathrm{A}$. 103

Figure 4-35. Decrease in effective stiffness and natural frequency observed during accelerated fatigue life test of Blade B. 104 


\section{Chapter 6: Appendices}

Figure 6-1. Example of stream tube including rotating wake past wind turbine rotor.

Figure 6-2. Diagram of wind turbine blade sectioned into individual blade elements. The rotor angular velocity is $\Omega, r$ is the radius of the section, $d r$ is the differential section thickness, and $c$ is the section chord length. The lift, $\mathrm{F}_{\mathrm{L}}$ and drag, $\mathrm{F}_{\mathrm{D}}$ forces are found for every airfoil section.

Figure 6-3. Induced aerodynamic forces due to a relative wind velocity, $\mathrm{U}_{\text {rel. }} \mathrm{F}_{\mathrm{L}}$ is the airfoil lift force, $\mathrm{F}_{\mathrm{D}}$ is the airfoil drag force, $r$ is the radius of rotation measured from the hub center, $\Omega$ is the angular velocity of the rotor, $\alpha$ is the angle of attack, $\Phi$ is the angle of the relative wind, $\theta_{\mathrm{p}}$ is the section pitch angle, $a$ is the axial induction factor, $a^{\prime}$ is the angular induction factor, $\mathrm{dF}_{\mathrm{N}}$ is the differential normal or flapwise force and $\mathrm{dF}_{\mathrm{T}}$ is the differential tangential or edgewise force.

Figure 6-4. Illustration of the hinge-spring model of a wind turbine where $R$ is the blade radius, $U$ is the free stream wind speed, $e$ is the non-dimensional hinge offset, $K_{\beta}$ is the spring constant, $\beta$ is the flapping out of plane angle, $\Omega$ is the rotor angular velocity, $\Psi$ is the blade azimuth angle and the coordinate system $\mathrm{x}, \mathrm{y}, \mathrm{z}$ rotates with the blade.

Figure 6-5. Numerical blade model predicted edgewise and flapwise bending moments.

Figure 6-6. Example numerical blade response model predicted flapwise blade root bending moment as a function of blade azimuth angle at a rotor speed of $195 \mathrm{rpm}$.

Figure 6-7. Weibull curve fit for stress ratio bin $\mathrm{R}=0.0$

Figure 6-8. Weibull curve fit for stress ratio bin $\mathrm{R}=0.2$ 124

Figure 6-9. Weibull curve fit for stress ratio bin $\mathrm{R}=0.3$ 124

Figure 6-10. Weibull curve fit for stress ratio bin $\mathrm{R}=0.4$ 125

Figure 6-11. Weibull curve fit for stress ratio bin $\mathrm{R}=0.5$. 125

Figure 6-12. Weibull curve fit for stress ratio bin $\mathrm{R}=0.6$. 125

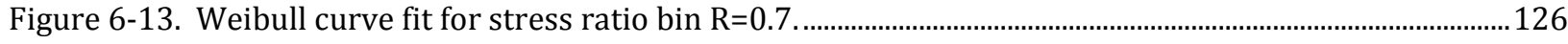

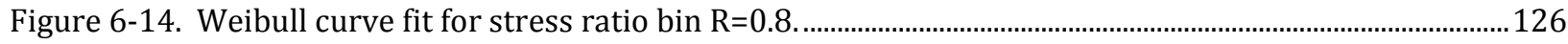

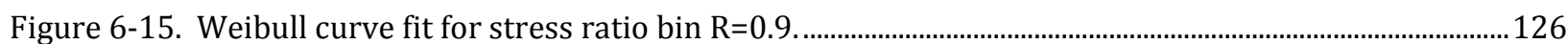

Figure 6-16. Blade profile design as provided by Gorman Controls, Ltd. 128

Figure 6-17. Comparison of chord length between all three blades at sections located 60 inches and 80 inches from the root 129

Figure 6-18. Measured cross sectional area variation between all three blades.

Figure 6-19. Chord length variation as compared to the ideal designed chord length for all three blades..... 130 Figure 6-20. Chord length variance as a function of radial position for all three blades compared to one another. 130

Figure 6-21. Chord length and cross sectional area variance compared to the ideal Clark Y profile for blade $\mathrm{C}$. 
Figure 6-22. Section thickness variation compared to the ideal Clark Y profile as a function of chord length in inches for blade C. Six profiles were measured in all.

Figure 6-23. Chord length and cross sectional area variance compared to the ideal Clark Y profile for blade B.

Figure 6-24. Section thickness variation compared to the ideal Clark Y profile as a function of chord length in inches for blade B. Six profiles along the blade were measured in all

Figure 6-25. Chord length and cross sectional area variance compared to the ideal Clark Y profile for blade A

Figure 6-26. Section thickness variation compared to the ideal Clark Y profile as a function of chord length in inches for blade A. Six profiles along the blade were measured in all.

Figure 6-27. Illustration of the six section profiles for blade A used in comparison to ideal Clark Y airfoil shape. Green is ideal Clark Y and red is the measured profile.

Figure 6-28. Illustration of the six section profiles for blade B used in comparison with the ideal Clark Y airfoil shape. Green is ideal Clark Y and red is the measured profile.

Figure 6-29. . Illustration of the six section profiles for blade $\mathrm{C}$ used in comparison with the ideal Clark $\mathrm{Y}$ airfoil shape. Green is ideal Clark Y and red is the measured profile. 136

Figure 6-30. All input accelerometer data for full scale fatigue test performed on blade A. Each test is shown with a red vertical line and a T\# indicator.

Figure 6-31. All tip accelerometer data for full scale fatigue test performed on blade A. Each test is shown with a red vertical line and a T\# indicator.

Figure 6-32. All leading edge strain gage data for full scale fatigue test performed on blade A. Each test is shown with a red vertical line and a T\# indicator

Figure 6-33. All middle strain gage data for full scale fatigue test performed on blade A. Each test is shown with a red vertical line and a $\mathrm{T} \#$ indicator 138

Figure 6-34. All trailing edge strain gage data for full scale fatigue test performed on blade A. Each test is shown with a red vertical line and a T\# indicator.

Figure 6-35. Strain gage output for the mid-span gages pair during test number three for blade B.

Figure 6-36. All leading edge strain gage data for full scale fatigue test performed on blade B. Each test is shown with a red vertical line and a $\mathrm{T} \#$ indicator

Figure 6-37. All trailing edge strain gage data for full scale fatigue test performed on blade B. Each test is shown with a red vertical line and a $\mathrm{T} \#$ indicator.

Figure 6-38. All trailing edge strain gage data for full scale fatigue test performed on blade B. Each test is shown with a red vertical line and a T\# indicator

Figure 6-39. All input accelerometer data for full scale fatigue test performed on blade B. Each test is shown with a red vertical line and a $\mathrm{T} \#$ indicator

Figure 6-40. All blade tip accelerometer data for full scale fatigue test performed on blade B. Each test is shown with a red vertical line and a $\mathrm{T} \#$ indicator. 
Figure 6-41. Signal offset between consecutive tests normalized by blade tip deflection for blade B...............142 


\section{Chapter 1. Introduction and Overview}

Wind energy has quickly become the renewable energy technology leader within the United States as the annual installed capacity continues to grow at a rate of $20 \%$ per year. In 2007 alone, over 3000 megawatts (MW) was installed, increasing the total capacity to over 25 gigawatts (GW) which is enough electricity to power the equivalent of 4 million homes and represents an offset in carbon dioxide production equivalent to 28 million tons (AWEA 2008). Other countries leading the wind industry include Spain, Germany and Denmark who all have experienced rapid growth in both installed capacity and production of large scale wind turbines. This rapid growth can be attributed to the economies of scale and aesthetic benefits granted to using larger and fewer wind turbines. Modern designs for large scale wind turbines include rated output powers of several megawatts and design lives of at least 20 years. On the other end of the power spectrum, small wind turbine systems have received a renewed interest for providing consumers with small scale power generation opportunities.

The designation of a small wind turbine is generally accepted within the U.S. to be a machine with a rated power output of 100 kilowatts or less (Manwell, McGowan and Rogers 2002). Turbines within this category are often very different in design when compared to their larger counterparts and the complexities of the operating environments for both large and small wind turbines, necessarily means that designs which work well on a large scale cannot simply be scaled down (Bechly 2000). Historically, due to government and public incentives related to the demand for decreased energy costs, large wind turbine designs have benefited from a vast amount of research and development in the last 25 years. Small wind turbine systems on the other hand, due mostly to their smaller market share in the past, generally have not received the same amount of careful scientific study. Currently, the demand for small wind turbine systems is increasing as a greater number of energy providers now support net-metering agreements which allow customers to sell the excess power generated by their wind turbines back to the grid. Furthermore, as demand for small wind turbine systems increases, further scientific research and development is needed to ensure these new turbines achieve a design life of at least 20 years. 
This thesis is aimed at filling the research and development vacuum existing between large and small scale wind turbines, specifically in the area of blade design. Some of the most important components of a robust small wind turbine design are the blades since all power capture and structural loading originates at these surfaces. Large scale wind turbines use fiber reinforced composite blades but the infrastructure cost associated with advanced composite construction methods for wind turbine rotors below $10 \mathrm{~kW}$ is prohibitive for units of this size. Wooden laminates are therefore often considered for blade construction as a less expensive alternative. The life expectancy and mechanical properties of such blades, however, are often unknown, depend on manufacturing processes as well as materials, and are often left to be tested in the field. This project aims at answering these questions on a new proposed wood blade design manufactured by Gorman Controls Ltd., of Prince Edward Island Canada.

A primary step in any thorough scientific investigation or experiment is the preliminary modeling of the system using known analytical techniques. This approach allows the experimenter to create a useful prediction of system performance to guide the creation of detailed experiments or data collection methods. To this end, the first objective of this project was the creation of a numerical code using the equations of blade element theory and the hinged spring offset model to define the blade flapping equation and predict loads and deflections at the hub, as well as define the rotating and non-rotating natural frequencies of each blade. These results were used as a guideline to design an experiment to collect strain values near the hub of each blade. This effort was completed using the technical communication software MATLAB which allowed rapid code iteration and visualization of system performance.

The second objective consisted of measuring the in-field loading parameters for the wind turbine rotor, as compared to the numerical predictions. The process of measuring the rotating blade root bending moment is inherently difficult because the transfer of data from strain gages located on the roots of the blades must occur in real time. The rotating nature of a wind turbine obviously complicates this process, and therefore, in order to avoid working with complicated slip rings and direct wire methods, a wireless data 
acquisition device was developed. The data was collected using strain gage bridges mounted directly on the blades and was transmitted using a wireless embedded transmitter, built in-house, and attached to the hub of a prototype turbine. The purpose of this system was to provide in-field data which was used to characterize the blade loading and frequency spectrum for a particular turbine and its site. Unfortunately, due to an unforeseen design flaw relating to the yaw bearing of the first prototype, the turbine fell from its tower before any useful in-field data could be gathered.

Not having the ability to collect the in-field loading parameters for the wind turbine provided a new challenge for this project, but this shortcoming was ultimately overcome through careful modeling and by reviewing the work of other researchers. This work was guided primarily by the work of Jayantha Epaarachchi and Philip Clausen who demonstrated that the stress cycles experienced by the blades of their research $5 \mathrm{~kW}$ wind turbine closely followed a Weibull distribution and furthermore these cycles could be statistically estimated with high accuracy without using a complete set of in-field loading parameters (Epaarachchi and Clausen 2006). A statistical estimate of the actual stress cycles experienced by the blades of the Gorman Controls wind turbine was therefore created based on a Weibull curve fit of local wind data and this data set was used to guide the creation of a fatigue loading spectrum to be used in an accelerated life test.

In parallel with determining an estimate for the fatigue life of the blades, a manufacturing verification study was conducted to ensure that each blade conformed to the designed Clark-Y airfoil profile selected for use. This was completed by utilizing a three dimensional laser part scanner which had the ability to capture the blade's profile and store this data as a rendered three dimensional object. This solid model was then compared to the designed airfoil shape at multiple cross sections and the deviation of these observed profiles from their theoretical counterparts was recorded. While the chord length at corresponding sections remained fairly constant, section thickness values were observed to deviate by as much as $20 \%$ when compared to an ideal Clark Y airfoil and this deviation can be attributed to the hand shaping process by which each wooden blade was made. Furthermore, this deviation was concluded to have a small effect on the structural integrity of each blade 
since all three blades measured were observed to be thicker and thus stiffer than the ideal Clark Y profile. While this deviation would affect the aerodynamic performance of the blade, this effect was outside the scope of this project and was not investigated.

When any type of fatigue analysis is performed on a structural component, the first task to be completed is the careful documentation of the material properties of that component. As noted, the blades for the Gorman Controls' wind turbine are fabricated using wooden laminates and in order to correctly model each blade's response to typical wind conditions, it was important to first quantify the material behavior of this composite laminate. Following the correct testing procedures outline by the American Society of Testing and Materials for wooden specimens, small coupons were cut from the wooden laminate blades (ASTM D-143 2002). These specimens were then subjected to several tensile experiments whereby the Young's Modulus and Poisson's Ratio were measured and used to describe the orthotropic behavior of this material. These values were then incorporated in the numerical performance model discussed previously to more accurately predict the typical response and stress state of the wooden blades at given wind velocities.

Using the material property data and stress response model previously mentioned, a full scale cycle test to determine the typical response of the laminated blades to a 20 year accelerated fatigue life test was performed. A mechanical driving fixture which attached to the blade root was built in order to induce an excitation at the blades first fundamental frequency and reproduce the accelerated stress state required for the test. In order to determine the number of appropriate fatigue causing cycles for the blades, a statistical study was completed using rainflow cycle counting on a year-long wind measurement spectrum obtain from Gorman Controls Ltd (ASTM E-1049 2005). This study counted the number of fatigue related cycles based on the wind spectrum and binned each cycle according to the respective range and mean amplitude. These wind cycles were then correlated to equivalent stress cycles at the root of the blade and the amount of cumulative damage experienced by the blades during a 20 year lifespan was predicted using methods described by Bond and Ansell to construct constant life curves and stress-cycle (S-N) graphs (Bond and Ansell 1998). Two blades provided by Gorman Controls Ltd. were tested 
with the fixture and each were driven at the first natural frequency with a prescribed amount of tip deflection in order to achieve the desired alternating stress state for a 20 year accelerated lifespan. Both blades experience a modest decrease in both frequency response and stiffness, however, both blades survived the accelerated life test thus demonstrating the effectiveness of the laminated wood at providing a structurally capable wind turbine blade for a 20 year period of use.

There are six chapters in this thesis including the introduction and appendices. Chapter 2 describes the current designs for small wind turbines and discusses the state of the art testing methods and practices for these systems. A discussion of the analytical methods for determining the fatigue life of a wooden composite and the numerical code created to model this response is provided in Chapter 3. The experimental equipment and procedures as well as a description of the measurement techniques and post-processing methods are outlined in Chapter 4. The summarized results, conclusions and recommendations are provided in Chapter 5. Chapter 6 contains the appendices. 


\section{Chapter 2. Small Wind Turbine Systems, Current Practice and Testing Methods}

Current small wind turbine systems are becoming increasingly popular and designs for these systems are often completed without the rigorous engineering analysis typical of their larger counterparts. The analytical theory that guides the design of a large wind turbine can, however, be used just as effectively for small wind turbines and this chapter outlines the current practical execution of this idea within the wind community. While there are numerous designs for small wind turbines, this paper will focus on the horizontal axis, three bladed designs since the Gorman Controls Ltd. wind turbine is of this class. To properly investigate the design and fatigue life of the blades for the Gorman Controls' small wind turbine, a study of the basic design and loading of a wind turbine must be completed. Study of the current state of the art testing and experimenting methods must also be studied. This chapter outlines the careful study of these topics.

\subsection{SMALL WIND TURBINE SYSTEMS}

\subsubsection{Typical Small Wind Turbine Components}

A typical small upwind horizontal axis wind turbine (HAWT) with three blades is shown in Figure 2-1. There are eight main components for a small wind turbine as identified in the figure. The blades are typically made from either a fiber reinforced plastic material such as fiberglass or, as is the case with the Gorman Controls' Wind Turbine, they can be constructed using wooden laminates. The blades are connected to the turbine at the hub via bolts installed through the blade material or they may be attached with studs embedded in the blade itself. This attachment is a critical load path since all aerodynamic loads pass through this joint. The blades and hub are collectively known as the rotor. The rotor then attaches to the gearbox or drive-train with a large bearing and shaft which can support both the thrust and bending loads imposed by the spinning rotor. The drive-train is then connected to the electrical generator with a hardened steel shaft. In some designs, the gearbox is removed from the turbine and the rotor is directly connected to the generator. This configuration is known as a direct drive system. The gearbox and 
generator are usually contained in a housing known as the nacelle which offers protection from the elements. The nacelle then attaches to the tower with another large suitably sized bearing which allows the turbine to yaw freely or rotate as the wind changes direction. This yaw control is performed by the tail vane located on the back of the wind turbine. As the wind changes direction, the tail vane forces the turbine to orient itself into the wind which ensures the wind turbine is producing power at an optimum level. This design allows the turbine rotor to be located upwind of the tower which decreases tower related losses in performance and gives the wind turbine the upwind designation.

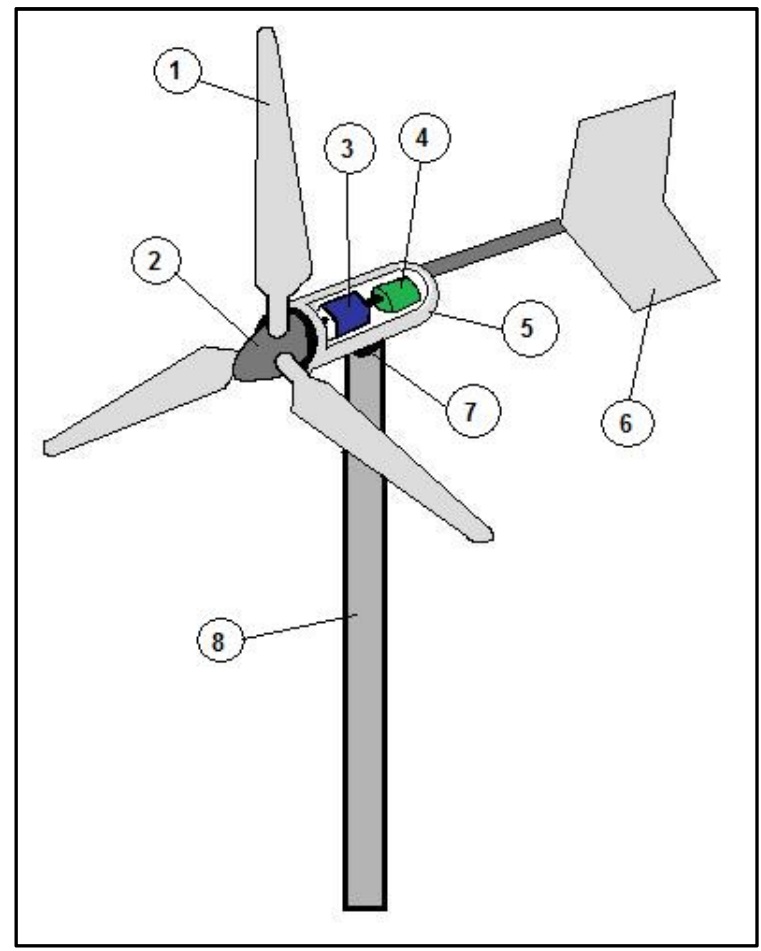

Figure 2-1. Typical components of a small wind turbine system: 1) Blades, 2) Hub, 3) Gearbox/Drivetrain, 4) Generator, 5) Nacelle, 6) Tail Vane, 7) Yaw Bearing, 8) Tower.

A component not described in Figure 2-1 which is still vital to proper operation is the overspeed control system. This system prevents the machine from operating at a speed higher than is acceptable according to the structural design of the wind turbine and thus prevents the rotor from spinning in high winds which could cause damage to the turbine. The over speed control is typically handled one of three ways; passive stall control, passive yaw control or incorporated tip brakes 
Passive stall control involves creating blades with a specific twist which controls the angle of attack of the airfoil. At high speeds, due to the fixed angle of attack, the blade will begin to shed wind and the rotor speed will not increase. Passive yaw control involves using an offset tail vane to manually turn the rotor out of the wind. This motion is known as furling and serves to decrease the aerodynamic efficiency of the wind turbine, thus slowing the spinning rotor. This design type is a passive mechanical system and operates based on predicted forces created by the wind reacting with the rotor and the tail vane. The last system involves adding flaps on the tip of each blade which orient themselves tangentially to the circle mapped by the blade tips during normal operation. In the event of higher than acceptable winds, the tip brakes can be deployed and they fold outwards, akin to the flaps on an airplane wing, in order to create high drag which then slows the rotor.

Small wind turbines can be used for both off-grid purposes such as battery charging and for heating homes or they may be used for feeding power back into the grid, a configuration known as net metering. Regardless of the end use for the system, the blades on a small wind turbine represent one of the most important components because all power extracted from the wind is based on their design. It is therefore important to first understand where the loading originates on the blades and then quantify this loading so the blade may be properly designed.

\subsubsection{Types of Loading on the Blades of a Small Wind Turbine}

Before estimating the magnitude of the loads on a small wind turbine blade, it is important to first understand the type of loading typical of these structures. As described by J. Manwell, a load is a general reference to any force or moment which may act upon the blades of a wind turbine (Manwell, McGowan and Rogers 2002). These loads may be static, which refer to a non-time varying load such as wind hitting a stationary blade. It is also possible for a wind turbine to experience steady non-time varying loads such as a rotor spinning in constant velocity wind. The most common type of load acting on a wind turbine is a cyclic time varying load. This type of load is akin to the rotating blades constantly moving in both the flap-wise and edge-wise directions in response to random changes in wind velocity. Because this type of load represents the majority of the loading 
spectrum, special designations are given to loads which occur in a periodic manner. A perrevolution designation of $1 \mathrm{P}, 2 \mathrm{P}$ etc. designates the number of times a particular load occurs during one revolution of the rotor. Other loading types include transient loads, referring to a load which is created in response to a short term event such as applying the tip brakes to stop the rotor from spinning. Impulsive loads are time varying loads of large amplitude which occur very quickly. A foreign object impacting the blade would be an impulsive load. Due to the inherent random nature of the wind, stochastic loads are very common for a wind turbine blade. Stochastic loads are time varying loads and arise when the wind is turbulent. A final type of loading is resonant induced loading which occurs when a wind turbine operates near one of its natural frequencies. This type of loading must be avoided during all operating conditions because the dynamic response due to excitation at a particular frequency may be very high and can quickly damage a structure. All seven types of loads presented above derive their origin from three main sources as listed in the following section.

\subsubsection{Sources of Loading on the Blades of a Small Wind Turbine}

Under normal and extreme loading conditions for a small wind turbine system there are three main categories from which all loading originates. Hansen describes these three categories as: aerodynamics, gravity, and dynamic interactions (Hansen and Martin 2008). Each category contributes to the overall loading spectrum and each must be properly considered for a robust and reliable wind turbine design.

\subsubsection{Gravitational Loading}

During operation, the blades on a wind turbine experience cyclic loading due to the Earth's gravitational field. During one rotation, the root of a blade, specifically the leading and trailing edges, will experience a tension-compression cycle due to the weight of the blade. This loading may be seen in Figure 2-2. While this cyclic loading is significant for larger machines and can influence the fatigue life of the blades, in general, the blades of a small wind turbine are significantly lighter than a megawatt class machine and thus experience relatively low amplitude loading due to gravity. Gravitational loading must still be 
considered for a small wind turbine but the fatigue life is often not affected by this source of loading (Epaarachchi and Clausen 2006).

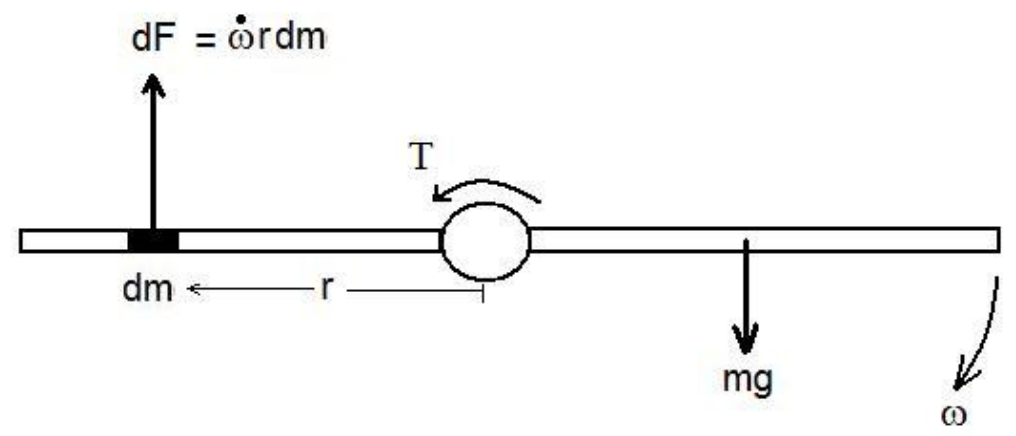

Figure 2-2. The loading on wind turbine blades due to gravitational and inertial forces.

\subsubsection{Aerodynamic Loading}

The most obvious source of loading on the blades of a wind turbine is the wind itself. These loads are directly related to the power production of the wind turbine and can create all seven types of loading as described in Section 2.1.2. When an airfoil is subjected to a relative wind velocity, a component of lift and drag are induced due to the aerodynamic properties of the profile. Figure 2-3 shows the standard nomenclature when describing the lift and drag forces created by an airfoil. When a wind turbine is operating in high wind conditions, the lift component force is of critical importance and when a wind turbine is stationary, the drag component is a primary consideration (Manwell, McGowan and Rogers 2002). The most critical loads derived from the aerodynamics of a blade are the loads which are created during very high wind conditions and the cyclic loads which may contribute to fatigue damage. 


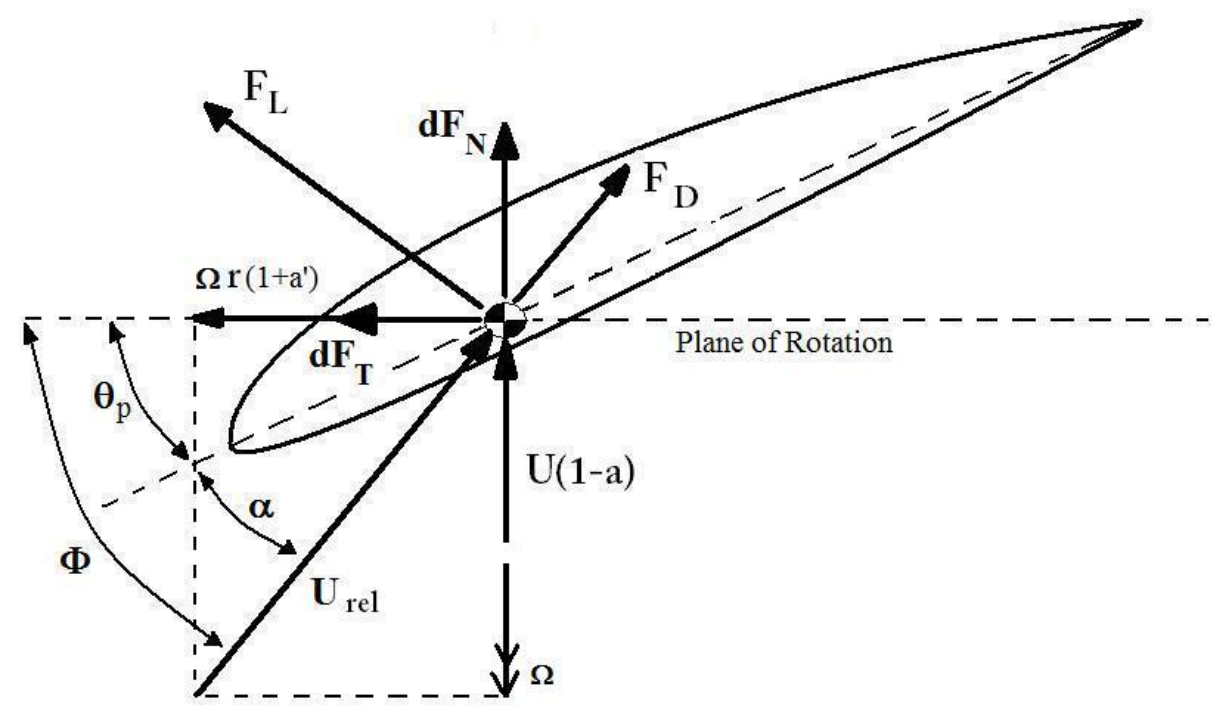

Figure 2-3. Induced aerodynamic forces due to a relative wind velocity, $\mathrm{U}_{\text {rel }} . \mathrm{F}_{\mathrm{L}}$ is the airfoil lift force, $\mathrm{F}_{\mathrm{D}}$ is the airfoil drag force, $\mathrm{r}$ is the radius of rotation measured from the hub center, $\omega$ is the angular velocity of the rotor, $\alpha$ is the angle of attack, $\Phi$ is the angle of the relative wind, $\theta_{\mathrm{p}}$ is the section pitch angle, $\mathrm{a}$ is the axial induction factor and a' is the angular induction factor.

\subsubsection{Dynamic Interactions}

Any type of motion created by gravitational or aerodynamic forces will ultimately induce loads on all other components of the wind turbine. These interactions must be carefully studied to avoid potentially dangerous dynamic interactions such as harmonic excitation of the blades close to their fundamental frequencies. The dynamic interaction between the mechanical control system and key wind turbine components like the blades are significant as well. As an example, if a spinning rotor is suddenly accelerated, as in the case of braking, the blades will experience a component of force due to the inertia of the rotating mass. This effect is shown in Figure 2-2 whereby a torque, $\mathrm{T}$, is applied to the rotor. The resultant force component in the plane of rotation is determined by the equation:

$$
d F=\dot{\omega} r d m
$$


where $\mathrm{dF}$ is the differential force in the plane or rotation, $\mathrm{r}$ is the radius of the rotation, $\mathrm{dm}$ is the differential mass of the blade at the particular radial location, and $\dot{\omega}$ is the angular acceleration of the section.

When describing certain accelerations of rotating bodies it is sometimes useful to refer to fictitious inertial forces. This intentional misnomer, which helps describe the effects of centripetal acceleration, is often referred to as the centrifugal force. When a blade rotates in a circle and is forced out of the plane of rotation by an increase in wind velocity, a component of the fictitious centrifugal force can be thought to force the blade back into the plane of rotation. This centrifugal force can be shown to be:

$$
F_{c}=\omega^{2} r d m
$$

where $F_{c}$ represents the fictitious centrifugal force, $\omega$ is the angular velocity of the rotor, $r$ is the radius of the rotating mass, and $\mathrm{dm}$ is the differential mass of the blade at the particular radial location. An illustration of this force may be seen in Figure 2-4 where the sine component of this centrifugal force pushes the blade towards the plane of rotation .

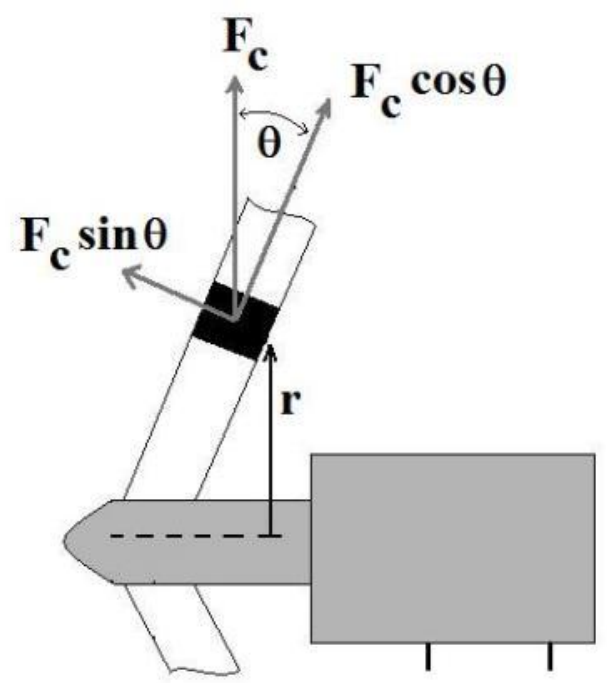

Figure 2-4. Side view of a wind turbine showing the fictitious centrifugal force and the derived components in the radial and normal directions (Hansen and Martin 2008). 
The effects of the centrifugal force, due to centripetal accelerations of the blade, are important to understand because this imaginary force effectively stiffens the blade during operation. This is achieved as the normal or sine component of the centrifugal force tends to push the blade back towards the plane of rotation which counteracts the tendency of a blade to flex away from the oncoming wind. By increasing the stiffness of the blade, this effect also increases the natural frequency of the blade which is beneficial because this increase often reduces the probability of exciting a blade at its first natural harmonic frequency.

\subsection{THE 10KW GORMAN CONTROLS WIND TURBINE}

\subsubsection{Basic Design of the System}

The design for the Gorman Controls' wind turbine differs from a traditional small upwind HAWT in several respects but the most apparent is that the generator is not a typical cylindrical induction generator but rather a pancake like axial flux permanent magnet generator. The other main components incorporated within this wind turbine are similar to the components defined in the previous section.

An axial flux generator resembles a thin spinning disk and derives its name from the orientation of the magnetic flux lines within the generator. Instead of being parallel to the radius of the blades, the flux lines are parallel to the rotation axis of the turbine rotor. The generator can be seen in Figure 2-5 during a stator coil test. The light green disks are the generator rotors and the permanent magnets can be seen on the insides of these plates. The stator mounts in between these plates and a single white stator coil being tested at the time can be seen in the figure. 


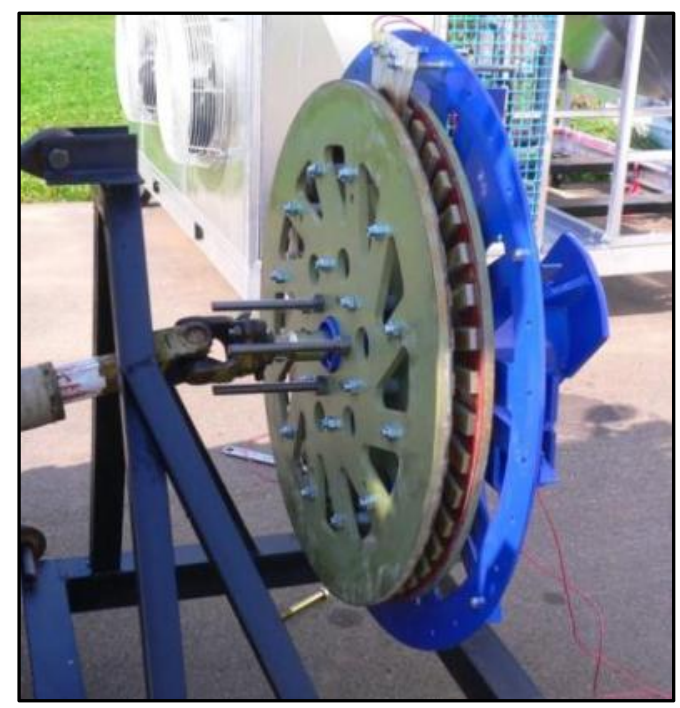

Figure 2-5. The axial flux generator for the $10 \mathrm{~kW}$ Gorman Controls' wind turbine mounted on a testing rig during a stator coil test.

In order to protect the wind turbine from spinning too fast, the Gorman Controls' wind turbine utilizes what is known as a tail furling mechanism to turn the rotor out of the wind. As can be seen in Figure 2-6, the tail is capable of rotating about a hinge towards the body of the wind turbine, a motion known as furling. When the wind velocity reaches a critical point, the tail begins to furl and slowly moves the rotor plane away from perpendicular to the wind and closer to parallel with the wind direction. If the wind velocity continues to increase, the tail will continue to furl until the plane of rotation is parallel to the wind direction.

The yaw and tail furl control is made possible by incorporating an offset between the blade's axis of rotation, the tower axis and the tail vane. Because the turbine is allowed to freely spin about the tower axis via the yaw bearing, a thrust force due to the wind reacting against the rotor is counterbalanced by the tail vane naturally trying to orient itself parallel to the direction of the wind. A demonstration of this offset may be seen in Figure 2-7. When a wind velocity is introduced at an angle other than 90 degrees to the rotor plane as shown in the figure, the resultant forces on both the rotor and tail vane create opposing moments about the tower axis. If the wind velocity reaches a critical point, the designed 
maximum wind speed for the wind turbine, the tail vane moment grows larger than the rotor moment and thus a rotation away from the wind will be observed by the rotor.

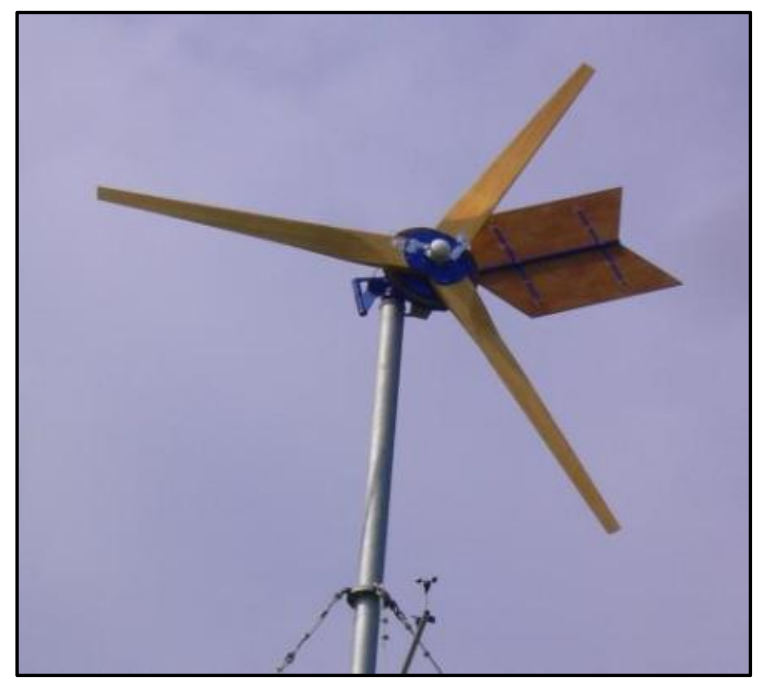

Figure 2-6. The Gorman Controls' 10 kilowatt wind turbine shown with the tail vane in the raised or furled position.

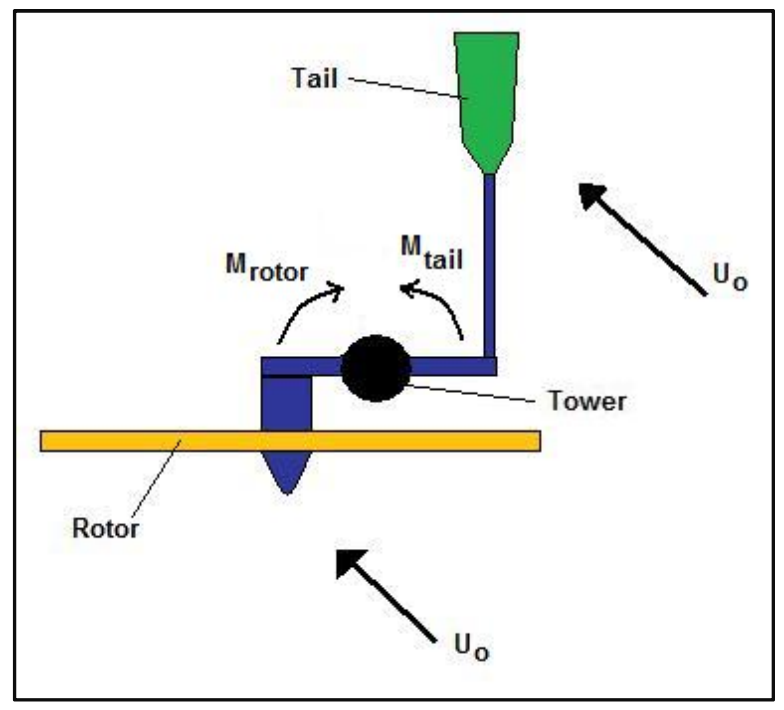

Figure 2-7. A top view of the Gorman Controls' wind turbine demonstrating the offset between the tail vane, the axis of rotation of the blades and the tower axis. $U_{o}$ is the relative wind velocity, $M_{\text {rotor }}$ is the moment induced by the rotor and $\mathrm{M}_{\text {tail }}$ is the moment induced by the tail vane. 


\subsubsection{Manufacturing the Blades for the Gorman Controls' Wind Turbine}

The blades for the Gorman Controls' $10 \mathrm{~kW}$ wind turbine are constructed using a simple wooden laminating technique. A local eastern white pine was selected for the blades since this wood is native to the east coast of Canada and was readily available for procurement. To begin, twelve foot lengths of one inch by four inch clear pine boards were cut to size and then run through a planar and then a jointer. This was completed to remove any bowing of the wood due to inconsistent drying and natural warping common in wooden boards. Epiglass HT900, a marine two part epoxy, was then used to laminate each board together and all boards were then stacked together with extra care taken to alternate the wood grain to avoid delaminating the glue lines. Clamps were applied to the laminated structure and the epoxy was allowed to dry for several days as per the manufacturer's instructions.

A Clark Y airfoil was selected for the power producing profile of the blade and a simple transition from the rectangular root to the airfoil profile was devised by Gorman Controls' employees. This simple transition was then drawn on the rectangular blade blank with section thickness indicator lines drawn every inch for the entire profile. These drawn profile lines were included on all four sides of the blank and then used as guides for carving the actual profile from the blank. All profile shaping was completed by hand using a hand router and hand planar. The finished blade profile was then sanded to a smooth surface and sealed using multiple coats of the same marine epoxy. Figure 2-8 shows a finished blade during the application of strain gages on the root which will be discussed in Chapter 4. 


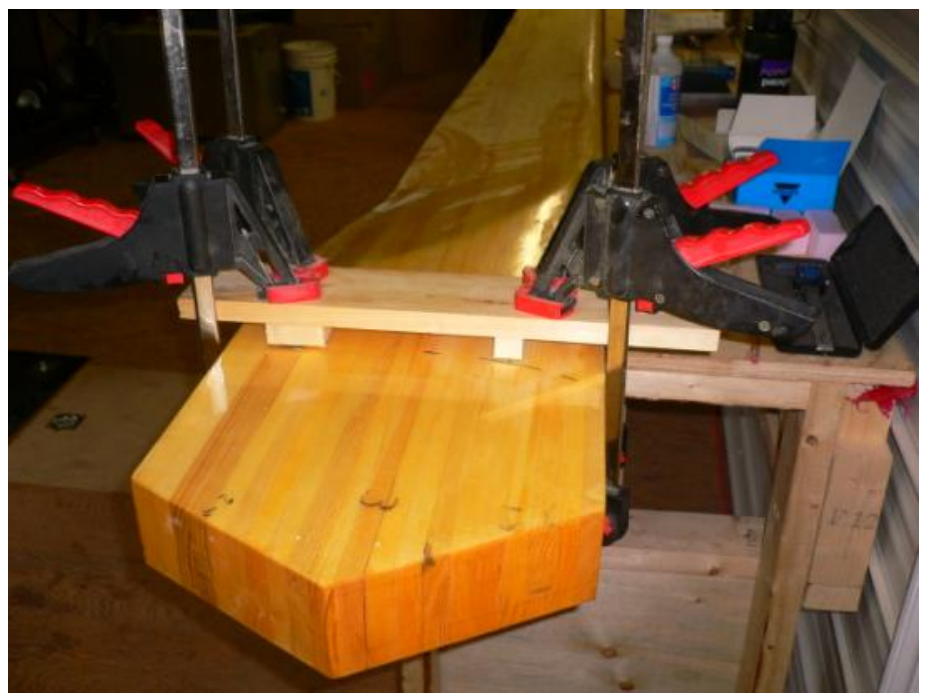

Figure 2-8. A finished wooden laminate blade for the Gorman Controls' 10kW wind turbine.

\subsection{SURVEY OF CURRENT SMALL WIND TURBINE BLADE TESTING METHODS}

There are currently many designs for small horizontal axis wind turbines on the market today. Typically, the blades for these machines are fabricated using either injection molded plastics or simple fiber reinforced plastics such as fiberglass, with E-glass being the most common type. While these fabrication methods do produce repeatable and reliable designs, the infrastructure cost associated with these manufacturing techniques is very high. In order to properly understand the benefits of using a wooden laminate instead of a traditional composite material, a survey of the existing blade design and testing methods has been completed.

\subsubsection{In-Field Blade Root Strain Measurement}

There is little published historical data on the response of small wind turbine blades to typical in-field loads before the year nineteen ninety. Following the increase in demand for small wind turbine systems during the mid nineteen nineties, research groups began to study the loading characteristics of the blades for these systems. Detailed studies to determine the principal loading conditions for small wind turbine blades has been 
competed by Kelley and McKenna (1996), Noakes, et al.(1993), Grant and Morgan (1996), van Hulle (1996) and Bechly and Clausen (2002).

Kelley studied the blades of an EXCEL-S $10 \mathrm{~kW}$ turbine which was used with a 3.5 meter tower. Three strain gages were attached to one blade of the wind turbine and were they used to evaluate a system for characterizing the turbulent loads experienced by the rotor. The stain gage signals were stored by an onboard data acquisition system which performed a rainflow cycle counting algorithm on the strain time history values. The binned cycle count values were then transmitted via a radio transmitter to a stationary PC based storage computer. Kelley concluded that variable speed small wind turbine systems accumulated fatigue damage at an increased rate when compared to a similar system with a constant rotor speed (Kelley and McKenna 1996).

Anderson, et al. focused on measuring the rotor shaft torque and blade root flapping moment from three blades on a 1.4 meter diameter rotor. These experiments were completed in a wind tunnel with the wind turbine operating in constant velocity wind. The strain gage data was captured via hard wired connections to a PC at high data rates made possible by advanced slip rings created by for the tests. Their work was focused on the effects of yawing a small wind turbine during operation. A conclusion from their work was that yaw misalignment contributes to blade flapping moments and thus increases the fatigue damage to the blades (Anderson, Noakes and Slater 1993).

Grant et al. worked on a small three bladed horizontal axis machine. Several strain gages were affixed to the blades of the wind turbine and the strain data was stored by an onboard data acquisition device. The device would store several hours of in-field operational data and then could be accessed via a data tether when the machine was taken down for maintenance. The difficulty of taking dynamic strain measurements from a small wind turbine blade was note in the study. Their main conclusion focused on the relationship between small variations in the blade's relative velocity and wind direction which can lead to dynamic stall effects (Grant and Morgan 1996). 
Van Hulle worked with a 5kW 3 bladed system developed in Sweden in order to validate the IEC 61400-2 safety code for small wind turbine systems. He took measurements from mid-span and root locations on several blades using a slip ring data acquisition system. Only two channels of data could be sampled in real time during each of his tests. Analysis of the data showed a strong correlation between the blade flap bending moments and wind speed events given by the IEC 61400- 2 code specifications (Van Hule 1996).

Bechly et al. studied the dynamic response of the blades on a $5 \mathrm{~kW}$ wind turbine. One of the $2.5 \mathrm{~m}$ long blades was instrumented with several strain gages along the entire length of the blade. Further instrumentation of the main shaft and other components gathered data pertaining to the torque, blade azimuth angle, wind speed, wind direction, turbine direction, turbine speed and ambient temperature. These measurements were sampled at a frequency of $200 \mathrm{~Hz}$ and the strain gage channels were recorded in the azimuth angle time domain. It was determined that the rectangular twist near the root of the blade lead to a coupling of the lead-lag and root flapwise bending moments and furthermore, these moments displayed a skewed once per revolution response to rotor accelerations (Bechley and Clausen 2002).

\subsubsection{Blade Fatigue Life Testing}

After finalizing the aerodynamic design of a wind turbine blade the structural design must be reviewed to ensure it will withstand all possible loading types during service. The stochastic nature of wind ensures that a blade will experience a significant number of fatigue causing cycles during its life span of 20 years and as a necessity a suitable fatigue

life analysis must be completed. Several studies have been completed in regards to small wind turbine blade fatigue life estimation. The following section presents the work of these authors.

P. Jamieson reviewed the design of a wooden epoxy wind turbine blade for a $15 \mathrm{~kW}$ wind turbine. Following a statistical review of the historical failure modes and fault characteristics of the machine he concluded that the main elements for proper design include appropriate wind and material data as well as tight control of blade geometry and 
material fatigue properties. Recommendations include better documentation of the turbulence intensities for a particular wind turbine site, more testing of the fatigue properties of wooden laminates and a tighter control of manufacturing processes (Jamieson 1990).

Current fatigue analysis of small wind turbine systems is hindered by the unavailability of a standard fatigue loading spectrum. Large wind turbines are analyzed using a standard spectrum known as WISPER/WISPERX but this model is inadequate for smaller designs because this spectrum was modeled based on operational data from large wind turbines with very different operating parameters than a small system (Have 1993). With this analysis void in mind, Sutherland has reviewed the available fatigue models for all wind turbines and emphasized the need to carefully study the wind distribution for site specific turbine placement, including statistical analysis of the rainflow counting methods with this analysis (Herbert 1994).

Noda et al. propose a simulation based method of determining the flapwise fatigue loading spectrum for a 27 meter rotor wind turbine. A cumulative fatigue damage model was created using a simulation model written by the authors and was based on operational data obtained from the wind turbine manufacturer. A synthetic wind speed profile created by the authors was used in combination with blade element theory to calculate the structural response of each blade. By assuming that a blade would instantaneously respond to given wind loading and by including turbulence intensity and wind shear, a conservative fatigue damage estimation was compiled and binned by stress level.

J.A. Epaarachchi et al. developed a fatigue loading spectrum for the blades of a small $5 \mathrm{~kW}$ wind turbine. Detailed measurements of the aeroelastic loads from a single blade were acquired simultaneously with wind speed measurements to show that the blade response does not instantaneously follow the wind profile. A methodology for determining the fatigue loading spectrum for a blade using the blade stress cycle counts from a rainflow analysis of the wind spectrum from a long term wind data set has been developed. The proposed method overcomes the necessity to obtain long term detailed operational data from a wind turbine before creating the fatigue loading spectrum. Both the short term 
detailed loading data and the long term wind profile values were found to be modeled well by a Weibull distribution. A joint probability relationship is proposed which creates a tabulated matrix of all fatigue cycles and stress levels binned by the associated stress ratio, R (Epaarachchi and Clausen 2006).

Bonfield and Ansell investigated the life prediction formula and rainflow counting algorithm in conjunction with Miner's rule to conservatively predict the fatigue life of wooden composites for wind turbine blades. By examining the hysteresis loops during a constant amplitude fatigue test of wooden samples taken from wind turbine blades, the dynamic property changes could be monitored. In tests at stress ratio values, $\mathrm{R}$, equal to negative one the modulus of elasticity was observed to reduce in a three stage process while the amount of energy dissipated per cycle increased as the cumulative damage builds up in the wood. It was observed that the rate of increase in hysteresis loop area accelerated throughout a test. By including the WISPERX fatigue loading spectrum in the analysis, accurate predictions for the fatigue life of wooden composites was completed (Bonfield and Ansell 1993). 


\section{Chapter 3. Analytical Methods for Determining Fatigue Life}

The blades of a typical small wind turbine system may experience many millions of cycles of loading and unloading throughout the lifespan of the machine due mostly to the stochastic or random nature of wind. These repetitive cycles often cause very small amounts of damage to occur within the material structures of the blades as each cycle stresses the root of each blade. Over time, a significant amount of cumulative damage can be accrued within the structure of the blade and this can lead to premature failure. This type of failure mode is known as fatigue damage and it plays a central role in the testing and certification of a wind turbine system. According to IEC standard 61400-2 for small wind turbine systems, a wind turbine must pass a duration test of at least 6 months of continuous operation without any type of failure in order to be certified (IEC1400-2 2006). While this 6 month time period may be sufficient to mitigate any short lived design flaws, customers now expect most small wind turbine systems to perform for at least 20 years, well beyond the certification lifetime. It is for this reason that a detailed study of the fatigue life, specifically one of at least 20 years, for the wooden blades of the Gorman Controls, Ltd. wind turbine has been carried out.

\subsection{FATIGUE OF WOODEN STRUCTURES}

It is well established within the engineering community that wood is a particularly good material at resisting progressive damage due to cyclic loading unlike more traditional engineering materials such as steel (Forest Products Laboratory 1999). This behavior is due in part to the natural evolutionary growth of trees as they needed to withstand large repetitious loading induced by the wind. While this natural ability to grow and flex with the wind has helped trees become some of the tallest organisms on the Earth, documenting this fatigue behavior from an engineering standpoint has seldom been preformed. This study has often been foregone due to the accepted notion that wooden structures do not fail due to fatigue and need only be designed to withstand the ultimate strengths of the wood. Furthermore, fatigue failures of wooden structures appear similar to ultimate 
strength failures and as a consequence these failure cases are not well documented. It is for these reasons that a survey of the current knowledge base was performed by the author. This information was then used to guide the creation of an accurate fatigue loading profile of the Gorman Controls' wind turbine blade.

\subsubsection{Engineering Definition of Fatigue}

Fatigue of mechanical components is considered to be the progressive inability of a structure to withstand loads which may be applied in a cyclic manner. An alternative definition known as progressive fracture is sometimes used to better describe the actual process occurring within a material (Juvinall and Marshek 2000). Regardless of the name, when a material undergoes cyclic loading small fractures begin to accumulate within the microscopic structure of the material. A crack front, the name given to the small fractures, usually originates at either a small imperfection in the material or a microscopic geometric stress riser and this front will continue to grow in certain directions based on the material's crystalline or microscopic structure. A crack will continue to grow unless it is arrested by a local change in material properties or geometry. Once a crack reaches a critical size determined by the ultimate strength of the material, a failure will occur.

\subsubsection{Fatigue Life (S-N ) Curves}

When classifying the ability of a material to resist progressive damage due to cyclic loading, a fatigue life or S-N curve is created. 'S' refers to the stress level at which failure occurs and ' $\mathrm{N}$ ' refers to the corresponding number of cycles associated with the failure. For example, if a stress level equal to the ultimate strength of the material is applied to a component, then the number of cycles predicted by the S-N curve will equal one. A material's S-N curve is created by testing the material in a standard fixture which submits the specimen to a constant amplitude load while the specimen is rotated. This provides a fully reversed loading on the specimen which means that each stress cycle has a zero mean and the material is subjected to equal positive and negative stresses. The load level is usually close to the ultimate strength of the material and the test is run until a failure occurs. The test is then repeated at decreasing load levels and the number of cycles to 
failure at each level is recorded. These data points may then be used to create a master curve for the material depicting the total number of cycles to failure at all load levels. Common ferrous materials such as steel often display an endurance limit which is defined as a stress level whereby more than $10^{7}$ cycles may be reached without a failure occurring. Typically, it is assumed that any load under the endurance limit may be repeated an infinite number of times without failure occurring. In reality, especially for non-metals such as composites and wood, a true endurance limit does not exist and is inappropriate for use (Forest Products Laboratory 1999).

\subsubsection{Alternating Stresses}

Under normal loading conditions a typical wind turbine may experience a number of loading and unloading cycles per one revolution of the blades. These stress cycles will not usually have a zero mean value and as such, it is necessary to related these values to an equivalent zero mean value so the $\mathrm{S}-\mathrm{N}$ curves created for the material may be used to predicted the fatigue life of the component. Figure 3-1 shows how these actual load cycles can be described by a mean stress, $\sigma_{\mathrm{m}}$, a maximum stress, $\sigma_{\max }$, and a minimum stress, $\sigma_{\min }$.

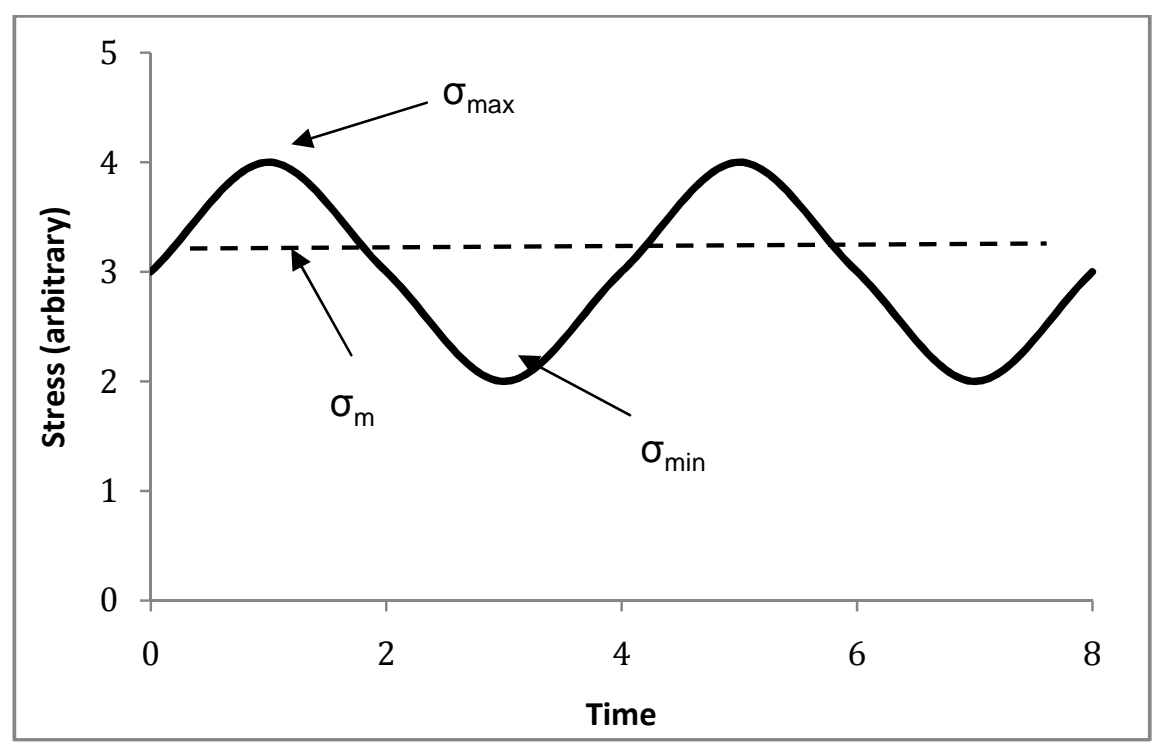

Figure 3-1. Illustration of non-zero mean alternating stress cycles. 
The amplitude of the alternating stress, $\sigma_{a}$, is defined to be:

$$
\sigma_{a}=\left(\sigma_{\max }-\sigma_{\min }\right) / 2
$$

And the stress ratio, $\mathrm{R}$, is defined as the ratio of maximum to minimum stress:

$$
R=\left(\frac{\sigma_{\min }}{\sigma_{\max }}\right)=\left(\frac{\sigma_{m}-\sigma_{a}}{\sigma_{m}+\sigma_{a}}\right)
$$

A useful equation known as Goodman's Rule can be used to equate these terms to an equivalent zero mean alternating stress, $\sigma_{e}$, which may be used in combination with the S-N curves and the materials ultimate strength, $\sigma_{\mathrm{u}}$ :

$$
\sigma_{e}=\frac{\sigma_{a}}{\left(1-\sigma_{m} / \sigma_{u}\right)}
$$

By using equation (3.3), the equivalent allowable stress level for a particular wind induced load may be calculated and related to the predicted number of cycles to failure given by the wooden material's S-N curve.

\subsubsection{Cumulative Damage Estimation}

When a component is subjected to a stress value less than the ultimate strength of the material, due to localized yielding at a microscopic level, a certain amount of damage is still accrued. While this behavior is quite complex, a linear cumulative damage equation known as the Palmgren-Miner Rule can be applied with relatively high accuracy to approximate the amount of damage sustained (Juvinall and Marshek 2000). The rule states that at a particular stress level relating to $\mathrm{N}$ number of cycles to failure, if a part is subjected to repeated loading at this level, every cycle will consume one part out of $\mathrm{N}$ total cycles of the life. This is approximated as:

$$
D=\sum \frac{n_{i}}{N_{i}} \leq 1
$$

where $n_{i}$ is the number of cycles at a particular stress amplitude and $N_{i}$ is the predicted total number of cycles to failure at the same stress amplitude. When the total damage as 
predicted by the S-N curve of the material and Equation (3.4) reaches a value greater than one, failure of the material is predicted.

\subsubsection{Fatigue Properties of Wood}

Very little information regarding the fatigue properties of wood is found in common engineering textbooks. Usually only the ultimate tensile and compressive strengths are listed with an approximate value of Young's Modulus in the fiber direction. Furthermore, these texts refer to the fatigue properties of wood as inconsequential when compared to the static loading strengths of the wood. In reality, wood can sustain structural damage due to repetitious loading and Lewis has shown that wood can ultimately fail due to fatigue (Lewis 1960). To understand why wood may fail to due repetitious loading it is necessary to understand the structural characteristics of wood from a microscopic level.

Wood is by definition is an orthotropic material because it behaves differently when separately loaded in the three principal directions; namely the longitudinal or length direction, the radial direction as defined by the lines perpendicular to the growth rings and within the growth ring's plane and the tangential direction as defined by lines tangent to the growth rings in the same plane. Figure 3-2 shows a sample wooden cube with the three principal directions labeled. 


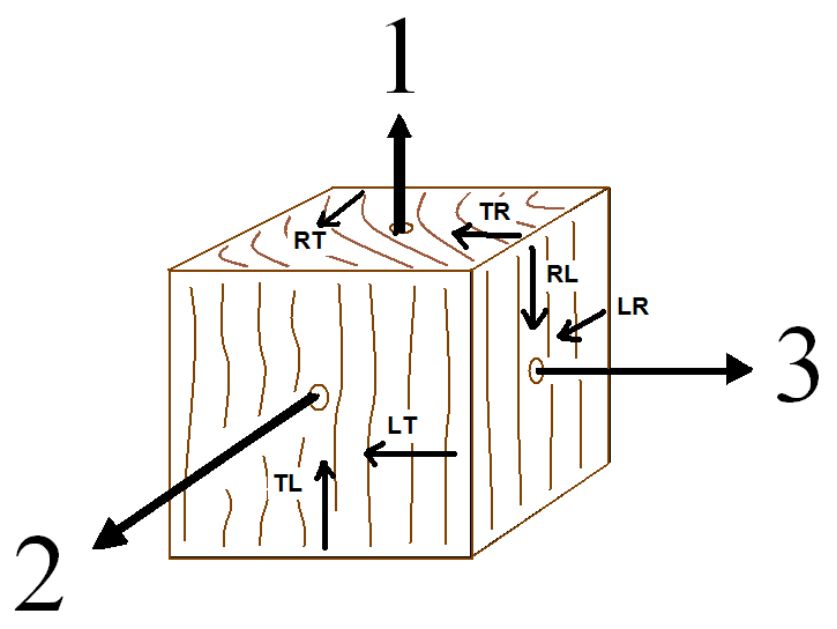

Figure 3-2. Illustration of the principal directions relative to the fiber direction in wood. 1) longitudinal direction, 2) radial direction, 3) tangential direction.

The longitudinal direction is considered the grain direction and is defined by rows of cells which are arranged in linear patterns. These patterns are often very complicated and contain many layers of cells. Each cell is then composed of many layers of microfibers made from the plant fiber cellulose. These microfibers are then bound in a matrix of lignin which is a complex polymeric adhesive (Kyanka 1994). This naturally gives wood a complex composite structure which exhibits both elastic and viscoelastic behavior. Elastic materials respond to applied stresses linearly and return to their original size and state if the applied stress is removed. Whereas a viscoelastic material's response to an applied stress is rate and time dependent and can be linear or non-linear depending on these two values. As a result, the behavior of wood in response to cyclic loading can be very complicated.

Furthermore, due to the orthotropic material properties of wood which include variable cell size and localized density gradients, the propagation of fatigue related cracks in wood is often unnoticed. This is due to the crack absorbing and deflecting mechanisms provided by the complicated cell structure of the wood. A crack front, as defined in fatigue analysis, created by repetitive loading is usually arrested after moving only a small distance in the 
structure. This is contrary to the crack propagation of crystalline metals where the front continues to grow as fatigue loading continues.

\subsubsection{Fatigue Crack Propagation in Wood}

There are six different fatigue crack propagation directions in wood and therefore the evaluation of each direction is necessary to properly understand the fatigue behavior of wood. Table 3-1 lists the six different directions of crack growth as defined by Kyanka. An illustration of these directions may be seen in Figure 3-2.

Table 3-1. Definition of six crack propagation directions for wood.

\begin{tabular}{|l|c|}
\hline \multicolumn{1}{|c|}{ Direction } & Abbreviation \\
\hline Longitudinal-Radial & LR \\
\hline Longitudinal-Tangential & $\mathrm{LT}$ \\
\hline Tangential-Radial & $\mathrm{TR}$ \\
\hline Radial-Tangential & $\mathrm{RT}$ \\
\hline Radial-Longitudinal & $\mathrm{RL}$ \\
\hline Tangential-Longitudinal & $\mathrm{TL}$ \\
\hline
\end{tabular}

The easiest cracks to arrest are ones which arise due to longitudinal loading. The LT and LR crack directions are oriented normal to the fiber direction of wood. Failures in this direction are not common due to the strong arresting capabilities offered by the fibers of the wood. Typically, a static fracture due to bending about the radial or tangential axes will resemble this fatigue failure mode and will not exhibit an observable crack propagation origin or complete separation of surfaces. Fatigue failure in the LT and LR directions are rare for these reasons.

Creation of cracks in the TR and RT directions move relatively un-impeded but rarely arise because wood is seldom loaded in these directions. Loading in these two directions would be akin to applying a torsional load about the longitudinal axis. Cracks can form when large torsional loads are applied but this type of loading is rare and fatigue failure due to this twisting seldom occurs. 
The most common directions for crack propagation are the RL and TL directions. The fibers of wood are parallel to these directions and the stacked cells allow cracks to propagate relatively easily. The material properties of wood perpendicular to the fiber direction are the weakest and as such, offer relatively few sites for cracks to stop. Structural designs using wood avoid placing loads in these directions but some loading is inevitable due to the Poisson effect. This effect is observed when a positive strain is applied to a material in a primary direction and a local contraction is observed in the transverse directions. Loading of a cantilevered beam like a wind turbine blade will produce these effects and therefore, these crack propagation directions are the most common for a wooden wind turbine blade.

A final note in regards to the fatigue of wooden structures relates to the gradual reduction in specific gravity for all commonly used wood species. Due to fast growth timber production, heavy fertilization and loss of old growth forests, the average density of wooden products is decreasing. Bodig et al. has shown that all wood strength properties are directly related to the density of the wood by a variable power law (Bodig and Jayne 1982). Therefore, as the material properties of wooden structures continue to decrease, the fatigue properties become more critical and the threshold of where fatigue failure will occur will be lowered.

\subsubsection{Derivation of S-N Master Curves for Wooden Laminate}

When estimating the fatigue life for any type of mechanical structure, proper derivation of material S-N curves is crucial. The blades of the Gorman Controls' wind turbine are made from eastern white pine which has relatively little information published in reference to the wood's fatigue life. Lacking the proper test facilities at Cal Poly to undertake a rotating fatigue life study of the laminated pine blade material, a derived S-N curve was created based upon careful study of existing literature in the field of wood fatigue.

Review of the work of Tsai and Ansell as well as the work of Bonfield and Ansell has shown that while there may be a large uncertainty in the actual fatigue strengths of various wood species, a general consensus seems to be that solid and laminated wood do not vary greatly 
in fatigue behavior (Bond and Ansell 1998) (Ansell and Tsai 1990). Furthermore, Ansell has shown that proper statistical analysis of ultimate tensile (UTS) and ultimate compressive strength (UCS) data allows the rapid generation of simplified constant life lines which offer a conservative estimation of the fatigue strength for wooden laminates. Careful testing of many samples of wooden laminates was performed to generate accurate S-N curves for beech, pine and poplar laminates. Bell curve shaped constant life lines were created based upon these tests which depict the full range of loading from $R=+1$ to $R=-1$ in both compression and tension. When the ultimate compressive strengths for the beech, pine and poplar samples were normalized, their respective fatigue behaviors were shown to correlate well. This allowed Bond and Ansell to create a generalized triangular curve with corners at the UTS and UCS to approximate a constant life diagram at stress ratio, R, equal to negative one. This proposed triangular approximation is shown to fit well inside the experimentally measured constant life diagrams. Using a $95^{\text {th }}$ percentile lower confidence limit to describe the ultimate strengths of the particular wooden laminate further adds to the conservative nature of the derived curve. The proposed master curve created by Bond and Ansell is given as:

$$
\sigma=\sigma_{c}[0.97-0.103 \log N]
$$

where $\sigma$ is the minimum allowable stress at $\mathrm{N}$ number of cycles, $\sigma_{\mathrm{c}}$ is the ultimate compressive strength of the wood, and $\mathrm{N}$ is the number of cycles to failure. This derived relationship was shown to correlate well with the experimentally measured curves. While certification of a particular wooden laminate for a guaranteed fatigue life would require proper measurement of the exact stress-cycle curve, this derived curve provides an accurate estimation using only the material properties of the wooden laminate.

Lacking the published S-N curves for the white pine laminate used within the Gorman Controls' wind turbine blade, this derived curve was chosen to approximate the desired S-N curve. Proper measurement of the ultimate strengths for the white pine laminate, specifically the ultimate strength in compression, was performed and this experimentally determined value was used to complete the $\mathrm{S}-\mathrm{N}$ curve to the pine laminate. This experimental testing methods and results used to obtain this value are described in 
Chapter 4. Using these ultimate strength values and Equation (3.5), the S-N curve for the eastern white pine laminate was created and is shown in Figure 3-3.

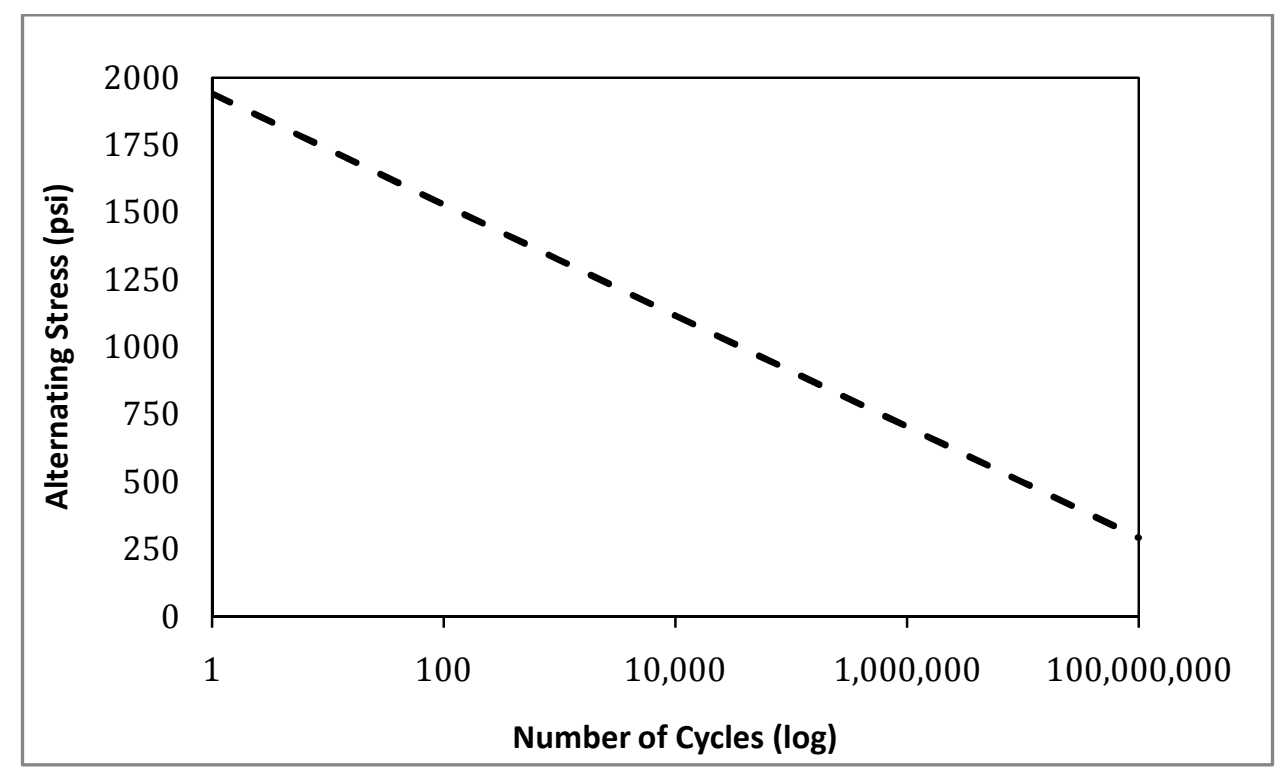

Figure 3-3. Derived S-N master curve for eastern white pine laminate at stress ratio $\mathrm{R}=-1$.

\subsection{CREATION OF ANALYTICAL WIND LOADING SPECTRUM}

In order to accurately predict the amount of fatigue damage a typical wind turbine blade may experience during its lifetime, the time loading history for the blade must be understood. As mentioned previously, fatigue causing cycles experienced by a rotating wind turbine blade are almost exclusively the result of fluctuating wind velocities. All loads experienced by the rotor originate from the stochastic nature of the wind influencing the structure. Thus, a proper study of the particular wind regime for a typical Gorman Controls' wind turbine must be completed. A year long time history documenting the 10 minute averages for wind speed and direction in 2004 was obtained from the Wind Energy Institute of Canada (WEICAN) for this purpose. The data was measured at a height of 30 meters, the tower height for the Gorman Controls' turbine, at WEICAN's facility on the northernmost tip of Prince Edward Island, Canada. This location is an exposed, flat region and is known historically for high winds. As such, this data set will represent a 
conservative measure of the average wind profile for Prince Edward Island, the primary installation region for the Gorman Controls' wind turbine.

\subsubsection{Rainflow Cycle Counting Method}

The need to monitor the life-cycle response of a structure based on a randomly applied loading pattern is a difficult problem. For steady loading, only the frequency and amplitude of the loading profile are needed to estimate the total damage accumulated by the structure under investigation. For randomly applied loads however, no pattern exists within either the time or amplitude signals. Many structures other than wind turbines are subjected to random loading throughout their lifetimes and it is for this reason that a method to analyze random signal time histories was created within the fatigue engineering community. This technique is known as rainflow cycle counting and was developed specifically to identify the amplitude and mean values from a time series of random signals (Downing and Socie 1982).

The details of the rainflow cycle counting method are fairly complex but the overall algorithm can be explained as follows. Local high and low signal values are identified as either a peak or valley by systematically polling the signal data and determining if the current value is between pre- determined thresholds. These thresholds determine if the cycle is to be considered a positively or negatively sloped half cycle. Figure 3-4 illustrates this method of measuring local high and low values and storing these for determining half cycle counts. The data points between A and B are stored as a positive half cycle while the data between $\mathrm{B}$ and $\mathrm{C}$ is stored as a negative half cycle. The algorithm computes all half cycles and then systematically pairs each positive and negative half cycle based on their respective amplitudes. Each cycle is then stored in a predetermined bin which describes both the range and mean of the stored cycle value. A complete rainflow cycle count thus creates a tabulated list of every cycle based on the mean and alternating amplitude for every matched cycle. By rainflow cycle counting a random signal time history such as wind data, the non-zero alternating stress values based on the wind profile can be related to a zero mean alternating stress and can therefore be used in conjunction with derived S-N curves. 


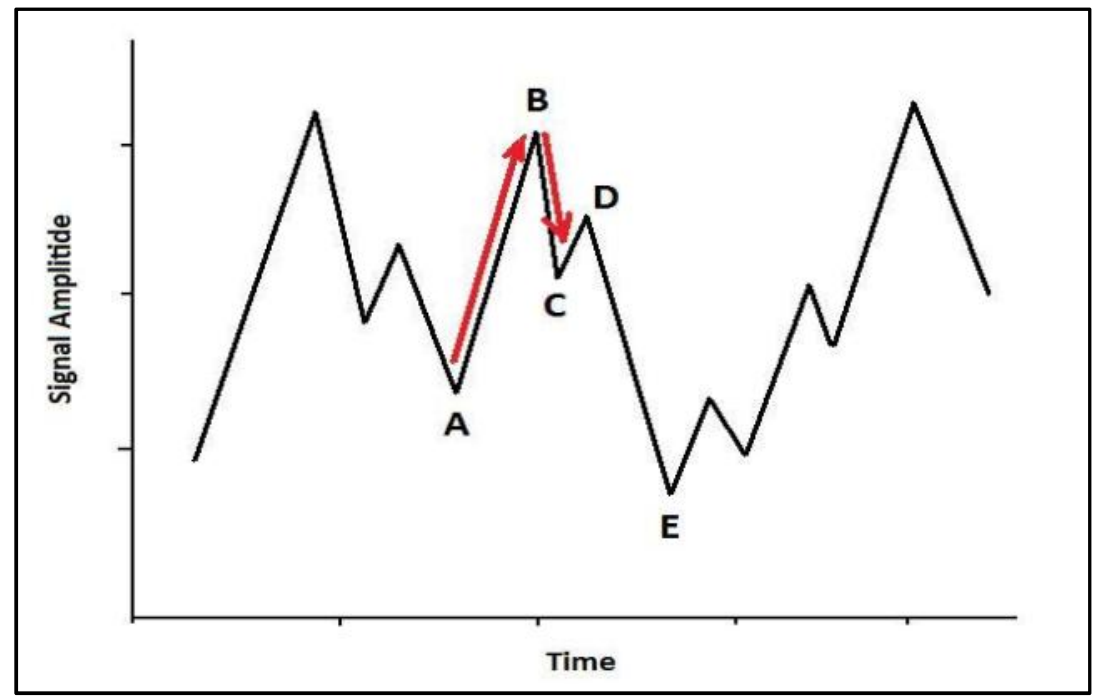

Figure 3-4. Illustration of rainflow cycle counting method of measuring half cycles.

\subsubsection{Rainflow Cycle Counting the Wind Spectrum}

A yearlong wind data set was obtained from the Wind Energy Institute of Canada (WEICAN) to begin the process of creating a fatigue loading spectrum for the blades of the Gorman Controls' wind turbine. This data set contained ten minute sample averages for windspeed, direction and standard deviation from a 30 meter high tower starting on January $1^{\text {st }}, 2004$ at 12:00 AM and ending on December 31st, 2004 at 11:50 PM. The tower is located on an exposed peninsula on the northernmost tip of Prince Edward Island, Canada and this location is known historically for having a high average windspeed.

\subsubsection{MATLAB Rainflow Cycle Counting Program}

To begin the process of counting the number of fatigue causing stress cycles for the Gorman Controls' wind turbine a MATLAB program was written to perform a rainflow counting algorithm. The purpose of this program was to take the raw spreadsheet data given by WEICAN and systematically count the number of fatigue causing cycles from this spectrum. To complete the task, three separate programs were written; two MATLAB functions and one script which used both functions to create a finalized rainflow matrix. Using methods 
outlined by Downing and Socie and the American Society of Testing and Materials a rainflow counting algorithm was created (Downing and Socie 1982) (ASTM E-1049 2005). The MATLAB programs may be reviewed in Appendix C.

The MATLAB programs accepted raw spreadsheet data files as input with the windspeed, standard deviation of speed and time stamp values stored in vertical columns. The program then completed a rainflow cycle count of the entire year long wind spectrum and generated a table report listing the total cycle counts binned by their respective mean windspeed value and alternating signal amplitude. The tabulated cycle count totals may be seen in Chapter 6.

\subsubsection{Results of Rainflow Cycle Counting}

Direct interpretation of the binned wind speed cycle counts is somewhat difficult to visualize so these tabulated values are often used to create a histogram which better illustrates the trends in the cycle totals. If the tabulated cycle counts are arranged into cumulative totals based on their respective wind speed bin value, a descriptive histogram may be created. Figure 3-5 shows the wind speed cycle totals per one meter per second wind speed bins as determined by the rainflow cycle count. This figure is a direct interpretation of the rainflow cycle count totals and shows an obvious skew in the data. This type of skew is common to wind speed regimes around the world and is approximated well by the statistical probability function known as the Weibull distribution (Epaarachchi and Clausen 2006). 


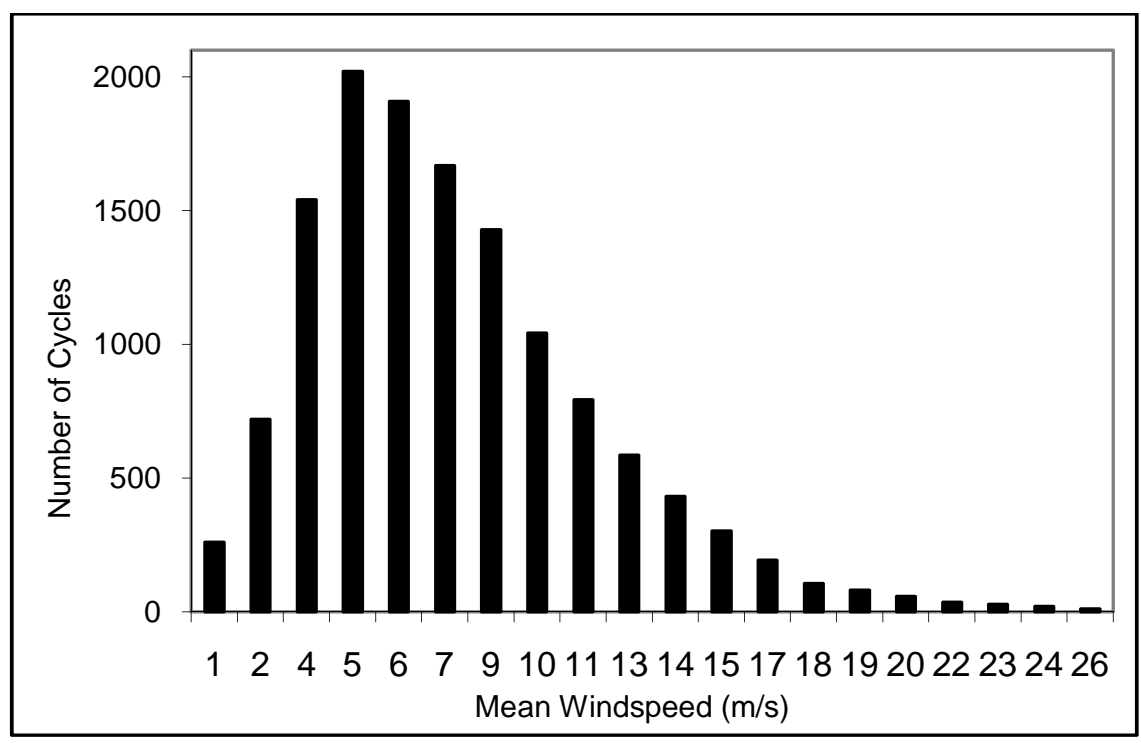

Figure 3-5. Preliminary results of rainflow cycle counting the WEICAN 2004 year $30 \mathrm{~m}$ tower data.

Simply counting the total number of wind speed cycles based on the obtained WEICAN field data is not sufficient for a detailed analysis of the complete wind regime because it is known the data represented only ten minute averages of the complete year long history. Further statistical analysis of the wind regime, specifically focusing on creating an explicit model of the wind distribution which could be used to predict the missing ten minute cycle history, was necessary if the desired stress related cycles were to be predicted. An explicit model of the wind regime using the Weibull statistical distribution was therefore created and the methods for doing this are described in the following section.

\subsubsection{The Weibull Distribution}

The Weibull distribution is a special case of the more generalized Pierson class III distribution common in statistical analysis (Devore and Farnum 2005). This distribution can be described by two functions; the probability density function (PDF) and the cumulative distribution function (CDF). The Weibull PDF indicates the fraction of time, i.e. the probability, for which the wind is at a particular velocity. This is given by:

$$
P D F=\frac{a}{b^{a}} V^{a-1} e^{-(V / b)^{a}}
$$


where $\mathrm{a}$ is the Weibull shape factor, $\mathrm{b}$ is the scale factor and $\mathrm{V}$ is the wind velocity. Therefore, the probability that the wind is between $V_{1}$ and $V_{2}$ is given by the function:

$$
P\left(v_{1}<v<v_{2}\right)=\int_{V_{1}}^{V_{2}} P D F d V
$$

The cumulative distribution function indicates the probability that the wind is equal or less than a particular wind velocity. The cumulative distribution function is simply the integral of the PDF over the interval from zero to $V_{i}$ and is given by:

$$
C D F=\int_{0}^{V_{i}} P D F d V=1-e^{-\left(V_{i} / b\right)^{a}}
$$

Describing a particular wind regime using the Weibull distribution is very useful because this allows statistical analysis of the wind data to be performed with good accuracy.

\subsubsection{Weibull Parameter Estimation}

In order to use the Weibull distribution as a means of approximating a wind regime, the scale, a, and shape, b, factors must be estimated. There exist several methods of performing this curve fit and a simple graphical method is presented here. Using the cumulative distribution function defined by Equation (3.8), and taking the natural logarithm of this equation twice, it can be shown that:

$$
\ln (V)=\frac{1}{a} \ln \left(\left(-\ln \left(1-(n-0.4) /\left(\sum n+0.2\right)\right)\right)+\ln (b)\right.
$$

where $\mathrm{n}$ is the cumulative number of wind cycles less than wind velocity V. Plotting the above equation with $\ln (\mathrm{V})$ on the $y$-axis and the right side of the equation on the $\mathrm{x}$-axis produces a nearly straight line. Figure 3-6 shows this relationship plotted for the WEICAN wind data. Performing a linear regression curve fit of the data allows the "a" and "b" factors to be calculated using the slope and intercept values produced. 


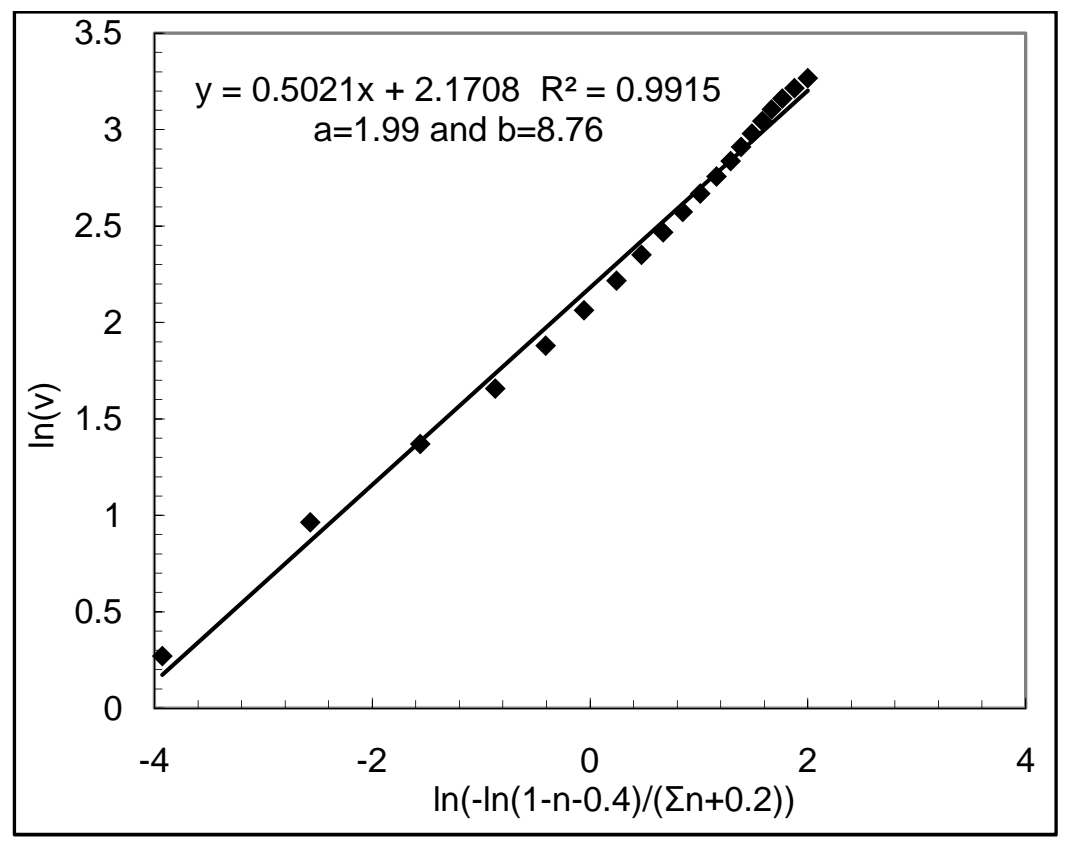

Figure 3-6. Linear form of the Weibull probability density function used to estimate the Weibull scale (a) and shape (b) factors for the WEICAN wind spectrum.

Using the equation for the Weibull probability density function and the derived shape and scale factors produced for the WEICAN wind data, an accurate curve fit was created. Figure 3-7 shows the curve fit closely approximating the relative frequency histogram of the WEICAN wind data. Having proved that an accurate representation of the wind profile can be achieved with the Weibull distribution, the deterministic stress cycles on the blade can now be calculated. 


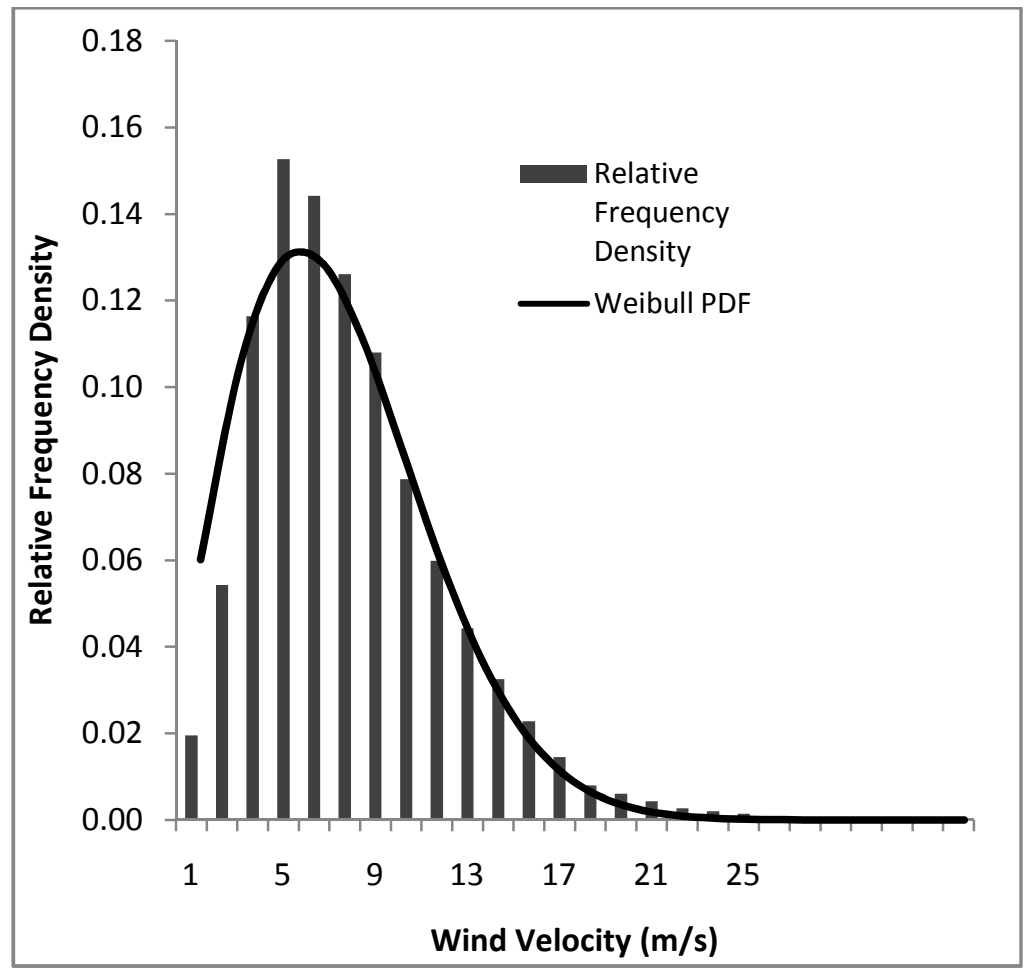

Figure 3-7 Weibull probability function with relative frequency density overlay showing curve fit for WEICAN wind data.

\subsection{NUMERICAL BLADE RESPONSE MODEL}

Before relating the predicted wind cycles experienced by the blades of the Gorman Controls' wind turbine to equivalent stress cycles, a relationship between the wind velocity and the critical stress on the blades must be determined. Ideally, the in-field loading data for the blades would be measured in parallel with the wind speed using strain gages and a data acquisition system similar to the experiments of Kelly and McKena (Kelley and McKenna 1996). This data would then relate the critical blade stress to each possible wind velocity. While an attempt to perform this measurement was completed by the author, there was an unfortunate failure of the main yaw bearing during a preliminary test and the wind turbine fell from the tower. This failure prevented any useful measurement of the infield loading to take place and as such, a new plan for determining the loads was devised. It was decided that a computer model of the operating wind turbine was to be completed and the predicted loading of the blades would be determined numerically. 


\subsubsection{Blade Element Momentum Theory}

When analyzing the energy which may be extracted from the wind by the blades of a wind turbine, the first step involves approximating the amount of thrust force imparted to the blades by the wind. A simple linear theory which approximates the wind turbine as an actuator disk which does not impart rotation to the fluid air flow is usually attributed to the German theorist Albert Betz (Betz 1926).

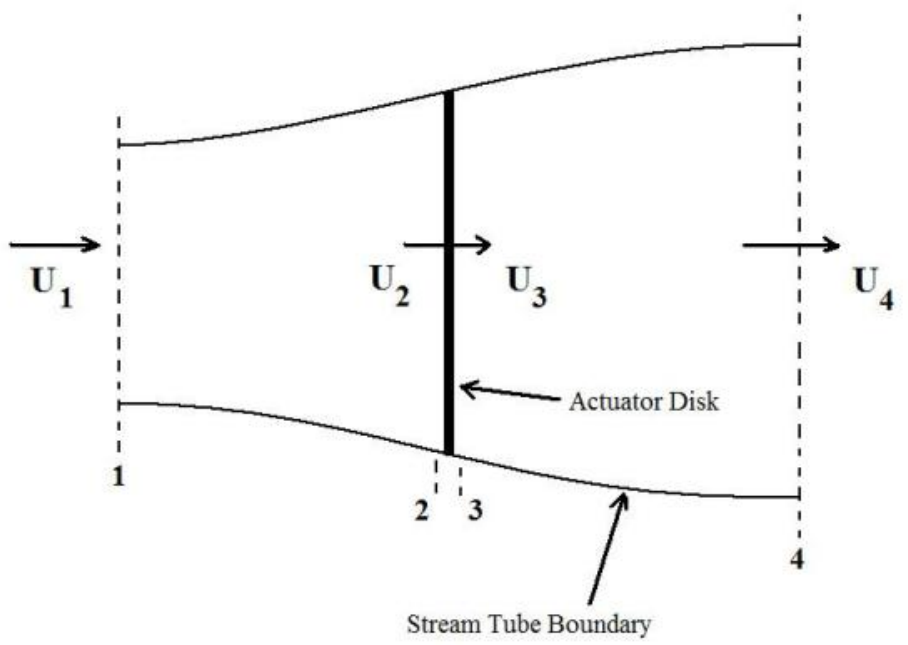

Figure 3-8. Linear approximation of a wind turbine as an actuator disk and stream tube where $U$ is the mean air speed at the four locations shown. (Manwell, McGowan and Rogers 2002)

If the conservation of linear momentum for a one dimensional, incompressible, timeinvariant fluid flow is applied to the actuator disk, the thrust on the actuator disk may be calculated as:

$$
T=U_{1}(\rho A U)_{1}-(\rho A U)_{4}
$$

where $\rho$ is the density of air, A is the cross sectional area and $U$ is the wind speed at each location indicated in Figure 3-8. A complete derivation of the thrust on the actuator disk is provided in Appendix $\mathrm{A}$ and the reader is referred to this derivation for the equations provided in the following discussion. The thrust on the actuator disk may be referred to using only the free stream wind speed, $\mathrm{U}_{1}$, by the equation: 


$$
T=\frac{1}{2} \rho A U_{1}^{2}[4 a(1-a)]
$$

where $\rho$ is the density of air, A is the area of the actuator disk, $U$ is the free stream wind velocity and the axial induction factor, $a$, is defined as the fractional decrease in wind velocity between the free stream, $\mathrm{U}_{1}$, and the rotor plane, $\mathrm{U}_{2}$ :

$$
a=\frac{U_{1}-U_{2}}{U_{1}}
$$

As derived in Appendix A, the performance of a wind turbine rotor may be expressed using a non-dimensional power coefficient, $C_{p}$, which is defined as the ratio of the rotor power to the total power available in the wind. This may be expressed using the axial induction factor as:

$$
C_{p}=4 a(1-a)^{2}
$$

To find the maximum value possible for the power coefficient, the derivative of equation (3.13)is taken with respect to $a$ and set equal to zero which yields $a=1 / 3$. Substituting this value for $a$ then gives the maximum rotor power coefficient as:

$$
C_{p, \max }=16 / 27=0.5926
$$

This derivation shows that the maximum power capture of an ideal wind turbine is limited to about $2 / 3$ of the total energy available in the wind. This linear model is only applicable for axial induction factors less than 0.5 because complicated turbulent flow occurs at values of $a$ greater the 0.5 which are not represented by this model. This ideal power capture is not possible in real life applications for three main reasons: rotation of the fluid flow occurs behind the actuator disk, a wind turbine has a finite number of blades and the drag on the airfoil is a non-zero quantity.

This simple linear theory can be improved upon by accounting for the rotating fluid flow created by the reaction of the fluid with the rotating blades. This analysis takes its roots from the generalized momentum theory first developed by Glauert to study the rotating propellers on early aircraft (Glauert 1935). The air flowing around the blades of a wind 
turbine can be approximated with a stream tube of radius $r$ and thickness $\mathrm{dr}$ which can have an angular velocity, $\omega$. A differential thrust force acting on an annular ring of area within the stream tube can be shown to be:

$$
d T=\rho U^{2} 4 a(1-a) \pi r d r
$$

Similarly, by applying conservation of angular momentum to the annular disk the differential torque imparted to the rotor can be shown to be:

$$
d Q=4 a^{\prime}(1-a) \rho U \pi r^{3} \Omega d r
$$

where $\Omega$ is the angular velocity of the rotor and the angular induction factor a' is defined as the ratio of the flow angular velocity, $\omega$, and the rotor angular velocity, $\Omega$ :

$$
a^{\prime}=\frac{\omega}{2 \Omega}
$$

A theory known as blade element or strip theory allows further investigation of the forces on a wind turbine blade by accounting for the aerodynamics of the blades. This analysis accounts only for the aerodynamic lift and drag forces at various angles of attack determined by the aerodynamic properties of the airfoils. The blades are divided into $\mathrm{N}$ sections and the lift and drag forces associated with each section are calculated depending on the angle of attack of the airfoil with respect to the relative wind velocity. It can be shown that the differential lift and drag forces are:

$$
\begin{gathered}
d F_{L}=C_{l} \frac{1}{2} \rho U_{r e l}^{2} c d r \\
d F_{D}=C_{d} \frac{1}{2} \rho{U_{r e l}}^{2} c d r
\end{gathered}
$$

where $C_{l}$ and $C_{d}$ are the lift and drag coefficients for the airfoil, $U_{\text {rel }}$ is the relative wind velocity and $\mathrm{c}$ is the airfoil chord length of the differential section. Realizing that these lift and drag forces will both contribute to the differential thrust and torque on the rotor, components of these forces can be calculated based on the angle of relative wind. The equations of blade element theory and momentum theory for the differential thrust and 
torque on the rotor may be equated to one other to provide a complete description of the forces acting on the blades. If the lift and drag coefficients are known for the specific airfoil, then the forces on each differential section of blade may be calculated and ultimately the blade flapwise and edgewise bending moments may be totaled based on the thrust and torque predicted. It is useful to note that for a reasonable approximation of the loads on the blades of a wind turbine, the above mentioned values and formulas are the only required parameters. A more detailed model which accounts for the additional degrees of freedom of the blades, is described the following section.

\subsubsection{Hinge-Spring Offset Model}

While the theory of blade element momentum theory accounts for the main aerodynamic forces acting on the blades of a wind turbine, two other sources of loading are neglected, namely the effects of gravity and rotor inertia. The hinge-spring offset model first developed by Eggleston and Stoddard, accounts for all forces which may act upon the rotor of the wind turbine by modeling the blade as a beam with hinges and springs allowing motion in the edgewise, flapwise and torsional directions (Eggleston and Stoddard 1987). The flexible blade is then approximated by a rigid uniform cross section beam, attached via springs to the hub with a hinged offset. The spring constants and offset distances are determined based on the material stiffness values for the blades. The derivation of the equations of motion for this system is time consuming and the reader is referred to the work of J.F. Manwell for the complete explanation (Manwell, McGowan and Rogers 2002). A review of this work is also given in Appendix $A$ and the reader is referred to this summary for the complete derivation of the equation of motion given below. In summary, the complete equation of motion for the rotating wind turbine blade can be show to be:

$$
\beta^{\prime \prime}+\left[1+\varepsilon+\frac{G}{\Omega^{2}} \cos (\Psi)+\frac{K_{\beta}}{\mathrm{I}_{\mathrm{b}} \Omega^{2}}\right] \beta=\frac{M_{\beta}}{\Omega^{2} I_{b}}-2 q \cos (\Psi)
$$

where $\beta^{\prime \prime}$ is the second azimuthal derivative of the blade flapping angle $\beta, \varepsilon$ is the hinge offset from the rotor center, $\Psi$ is the blade azimuth angle measured counter-clockwise from bottom dead center, $\mathrm{K}_{\beta}$ is the blade flapping spring constant, $\mathrm{q}$ is the yaw rate, and $\mathrm{G}$ is 
the gravity term $G=g m_{B} r / I_{b}$ where $m_{B}$ is the blade mass and $I_{b}$ is the mass moment of inertia of the blade.

Further manipulation of Equation (3.20) yields terms for the effects of gravity, wind shear, yaw rate and the hinge spring constants. While the complete solution of this equation was considered, a simplified model was used by the author whereby only gravity, hinge spring constants and flapping frequency calculations were performed. The yaw rate and wind shear terms were set to zero as per derivations provided by J. Manwell in Wind Energy Explained. The modified equations of motion were implemented in the numerical blade response model and this implementation is described in the next section.

\subsubsection{Implementation of MATLAB Blade Model}

Using the equations of blade element momentum theory and the hinged-spring offset model as described by Manwell, McGowan and Rogers, a numerical model of the wooden laminate blades response to typical wind conditions was developed using the technical computation software MATLAB. To begin, the geometric profile for the blade was input into the model with corresponding material properties of the wood. The nominal airfoil cross section design consists of a Clark Y profile and as such, appropriate lift and drag data was obtained from the National Advisory Committee for Aeronautics (NACA) (Silverstein 1934). Then, using the equations of blade element theory and the hinge-spring offset model, the predicted blade response was calculated. The differential normal and tangential forces observed were used to model each blade's root bending moments due to aerodynamic loading, gravity and inertia stiffening terms. Bending stresses were then calculated which corresponded to both the edgewise and flapping directions. The rotating and non-rotating natural frequencies for the blades were calculated using the theory developed by Mykelstad for general beam sections which creates a lumped mass model of the blade and iteratively solves for the system's Eigen values. A description of this method is presented in the book Theory of Vibrations (Thomson 1981). The hinged-spring offset model accounts for the tip deflection of the rotating blade as a function of the flapping angle, $\beta$, and the stress at the root of the blade was then calculated as a function of this 
deflection. Results of the model may be seen in the section below. The complete MATLAB script files and functions may be seen in Appendix C.

\subsubsection{Numerical Blade Response Model Results}

The MATLAB blade response model iteratively calculated the complete loading parameters for the blades on the Gorman Controls' $10 \mathrm{~kW}$ wind turbine using the complete equations of blade element momentum theory and the hinge-spring offset model. The numerical model predicted a maximum bending moment at the blade root in the flapwise direction of 650 foot-pounds, which corresponds to a predicted flap-wise bending stress of 122 psi. This value for the bending stress lies well under the ultimate stress of 4800 psi as given for typical white pine laminates in the US Department of Agriculture Wood Handbook (Forest Products Laboratory 1999). The predicted blade response bending moment curves may be seen in Chapter 6. Figure 3-9 shows the predicted tip deflection from horizontal of 3.4 inches at this stress level. A second order polynomial regression curve fit was created for this predicted response and can be seen in the figure. This regression curve was then validated during the full scale blade testing experiments which are described in Chapter 4.

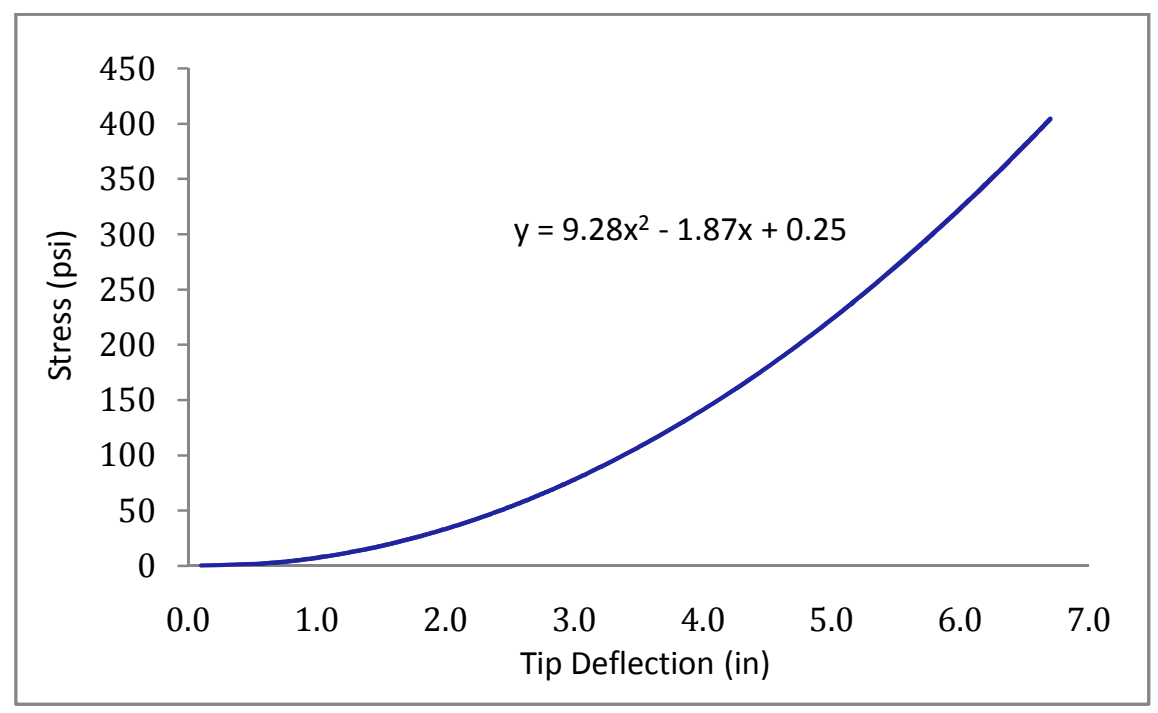

Figure 3-9. Numerically predicted blade root flapwise bending stress as a function of tip deflection out of the plane of rotation. 
The most important result of the numerical blade response model is shown in Figure 3-10. The normalized stress as a function of wind speed will be used to predict the critical stress at each windspeed bin as counted by the rainflow cycle counting exercise previously described. This result is the basis for the prediction of fatigue causing cycles for the blades.

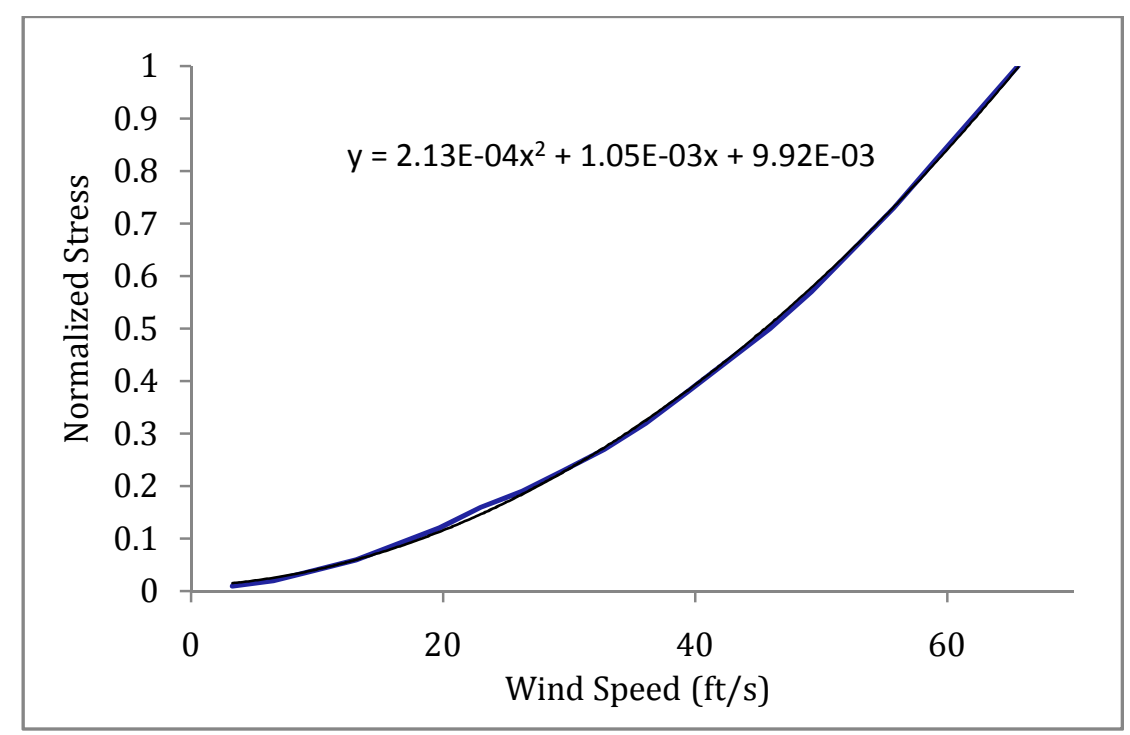

Figure 3-10. Numerically predicted normalized blade root flapwise bending stress as a function of wind speed.

The summarized results from the MATLAB numerical blade response model may be seen in Table 3-2. The first natural frequency of the blade during rotation is predicted to increase by $68 \%$ compared to the non-rotating natural frequency as would be expected due to inertial dampening. 
Table 3-2. Results of MATLAB numerical blade response model.

\begin{tabular}{|l|c|c|}
\hline & Value & Units \\
\hline Wind Speed Range & 0 to 45 & $\mathrm{mph}$ \\
\hline Maximum Rotor Speed & 275 & $\mathrm{rpm}$ \\
\hline $\begin{array}{l}\text { Maximum Flap-wise Bending } \\
\text { Moment }\end{array}$ & 650 & $\mathrm{ft}-\mathrm{lb}$ \\
\hline $\begin{array}{l}\text { Maximum Edge-wise Bending } \\
\text { Moment }\end{array}$ & 105 & $\mathrm{ft}-\mathrm{lb}$ \\
\hline Maximum Blade Tip Deflection & 3.4 & inches \\
\hline Non-rotating 1st Natural Frequency & 7.5 & $\mathrm{~Hz}$ \\
\hline Rotating 1st Natural Frequency & 12.4 & $\mathrm{~Hz}$ \\
\hline
\end{tabular}

\subsection{EQUIVALENT DAMAGE FATIGUE LIFE ESTIMATION}

\subsubsection{Stress Cycle Estimation}

A cyclic load is defined as a time dependent event whereby two loads of differing magnitudes are applied to a wind turbine blade at a specific rate. This is akin to the cyclic loading profile shown in Figure 3-1 where the two load magnitudes would correspond to the $\sigma_{\max }$ and $\sigma_{\min }$ values shown in the figure. Because a stress cycle experienced by a blade is directly linked to an equivalent wind speed cycle, a cycle from a higher wind speed to a lower wind speed or vice versa, as demonstrated by Fitzwater and Winterstein, the total number of stress cycles must also follow a Weibull distribution (Fitzwater and Winterstein 2001). Therefore, if an equation is known which describes the transformation of a particular wind velocity into a matching critical stress on the blade then the predicted number of wind velocity cycles can be related to equivalent stress cycles. Furthermore, the assumption that the blade root bending stress instantaneously follows a change in wind speed was used to provide a conservative estimate of the total number of cycles. This assumption follows the work of the above mentioned authors and the work of J. Epaarachchi et. al. who all use this idea to create a conservative estimate of the total number of fatigue causing cycles. Depending on how fast the wind changes velocity, an excitation of the blades first natural frequency is possible. However, because the WEICAN data set contained only 10 minute sample averages and no in-field loading data was 
obtained due to the prototype failure, no estimation of the actual frequency of this change can be made. To account for the loss of data, the statistical analysis of the wind data was intentionally made to overestimate the number of fatigue causing cycles which follows the work of the authors previously mentioned. This analysis is described below.

Using the derived relationship between windspeed and blade root flapwise bending stress predicted by the numerical blade response model as shown in Figure 3-10, the cumulative number of stress cycles was calculated at each wind speed bin. Knowing that the data set used to rainflow count the wind velocity spectrum contained only 10 minute sample averages; it must be assumed that a significant number of wind speed cycles were excluded during the counting process. Following the work of J. Epaarachchi et. al., a statistical analysis of the stress cycle counts was performed to account for these missing cycles.

The total cycle counts at each wind velocity bin were tabulated and used to create new Weibull probability density functions for predicted cycle totals at each stress ratio bin. Each stress ratio bin was calculated using Equation (3.2) and the alternating stress values predicted by the corresponding wind velocity cycle. By assuming that each stress ratio bin was well defined by a Weibull PDF, the corresponding shape and scale factor could be estimated using the graphical method defined previously. The Weibull shape and scale factors for each stress ratio bin were then calculated in this manner and are listed in Table 3-3.

Table 3-3. Weibull statistical parameters for stress ratio bins.

\begin{tabular}{|c|c|c|c|}
\hline $\begin{array}{c}\text { Stress Ratio, } \\
\mathrm{R}\end{array}$ & Shape, $\mathrm{a}$ & Scale, $\mathrm{b}$ & Cycle Totals \\
\hline 0 & 13.56 & 122.5 & 278 \\
\hline 0.1 & 2.44 & 54.4 & 2355 \\
\hline 0.2 & 2.18 & 45.7 & 14776 \\
\hline 0.3 & 1.78 & 52.8 & 45787 \\
\hline 0.4 & 1.23 & 42.7 & 125578 \\
\hline 0.5 & 1.09 & 33.8 & 210365 \\
\hline 0.6 & 0.91 & 25.7 & 541121 \\
\hline 0.7 & 0.88 & 21.1 & 402054 \\
\hline 0.8 & 1.65 & 54.8 & 334472 \\
\hline 0.9 & 2.21 & 70.8 & 98744 \\
\hline
\end{tabular}


As defined by J. Epaarachchi, Equation (3.21) predicts the number of stress cycles which may exist within a particular stress range:

$$
\text { Cycles }=\int_{\sigma_{1}}^{\sigma_{2}} f(\sigma) d \sigma N_{\text {total at } R}
$$

where $f(\sigma)$ is the stress function relating windspeed to critical stress and $N$ is the total number of cycles at a stress ratio, R. Using this equation and assuming each stress ratio bin also follows a Weibull distribution, the number of cycles at each stress level and stress ratio could be calculated. A more generalized form of Equation (3.21) can be created to express the cycle counts at any stress level and stress ratio, $\mathrm{R}$, using the cumulative distribution functions for wind velocity and stress ratio R previously determined. It can be shown:

$$
\text { Cycles }=\int_{\sigma_{1}}^{\sigma_{2}} f(\sigma) d \sigma N_{\text {total at } R} \int_{\mathrm{V} \text { range }}\left(1-\mathrm{e}^{-\left(\frac{\mathrm{V}}{\mathrm{b}}\right)^{\mathrm{a}}}\right) \mathrm{dV} \times \int_{\mathrm{R}_{\text {range }}}\left(1-\mathrm{e}^{-\left(\frac{\mathrm{R}}{\mathrm{b}_{1}}\right)^{\mathrm{a}_{1}}}\right) \mathrm{dR}
$$

where $\mathrm{V}$ is the wind velocity, $\mathrm{a}$ and $\mathrm{b}$ are the Weibull shape and scale factors for the wind velocity probability density function, $R$ is the stress ratio and $a_{1}$ and $b_{1}$ are the Weibull shape and scale parameters for the stress ratio Weibull probability density functions. Using this equation, the total fatigue damage causing cycles for the Gorman Controls' wind turbine blade were estimated. Figure 3-11 shows the Weibull curve fit for stress cycle counts for the stress ratio bin $\mathrm{R}=0.1$. Similar curves can be seen in Chapter 6 for all other stress ratio values

The final stress cycle counts for each wind velocity and corresponding stress level, binned by stress ratio, R, were calculated using Equation (3.22) and can be seen in Table 3-4. This table represents the complete fatigue loading spectrum for the entire yearlong wind regime from the WEICAN test site. 


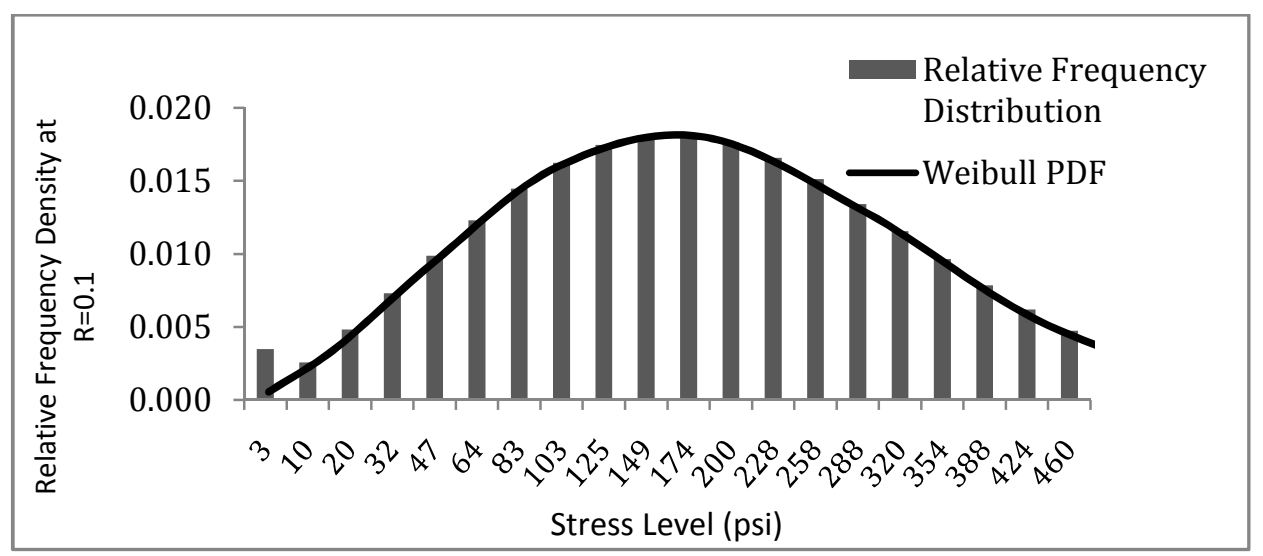

Figure 3-11. Weibull curve fit for stress cycles from binned data at $\mathrm{R}=0.1$.

Table 3-4. Final Rainflow counted fatigue damage stress cycles binned by normalized stress level and stress ratio, $\mathrm{R}$.

\begin{tabular}{|c|c|c|c|c|c|c|c|c|c|c|c|}
\hline \multirow{2}{*}{$\begin{array}{c}\text { Windspeed } \\
\text { Range } \\
(\mathrm{ft} / \mathrm{s})\end{array}$} & \multirow[b]{2}{*}{$\begin{array}{c}\text { Normalized } \\
\text { Stress }\end{array}$} & \multicolumn{10}{|c|}{ Stress Ratio, $\mathrm{R}=$ Min/Max stress } \\
\hline & & 0 & 0.1 & 0.2 & 0.3 & 0.4 & 0.5 & 0.6 & 0.7 & 0.8 & 0.9 \\
\hline 3 & 0.01 & 0 & 2 & 31 & 175 & 1920 & 4569 & 19791 & 17278 & 1596 & 75 \\
\hline 8 & 0.02 & 0 & 6 & 82 & 327 & 2156 & 4070 & 15443 & 12687 & 2665 & 203 \\
\hline 12 & 0.04 & 0 & 11 & 135 & 446 & 2191 & 3603 & 12588 & 9899 & 3421 & 344 \\
\hline 16 & 0.07 & 0 & 17 & 184 & 540 & 2141 & 3176 & 10447 & 7920 & 3967 & 491 \\
\hline 20 & 0.10 & 2 & 23 & 227 & 609 & 2044 & 2791 & 8760 & 6430 & 4344 & 636 \\
\hline 24 & 0.14 & 4 & 29 & 261 & 657 & 1922 & 2446 & 7397 & 5273 & 4578 & 775 \\
\hline 29 & 0.18 & 11 & 34 & 283 & 684 & 1787 & 2140 & 6277 & 4355 & 4689 & 902 \\
\hline 33 & 0.22 & 15 & 38 & 293 & 694 & 1647 & 1869 & 5349 & 3618 & 4697 & 1016 \\
\hline 37 & 0.27 & 18 & 41 & 292 & 687 & 1507 & 1629 & 4572 & 3019 & 4619 & 1111 \\
\hline 41 & 0.32 & 35 & 43 & 280 & 667 & 1371 & 1418 & 3919 & 2529 & 4471 & 1187 \\
\hline 46 & 0.38 & 40 & 43 & 259 & 637 & 1240 & 1233 & 3366 & 2126 & 4267 & 1241 \\
\hline 50 & 0.44 & 35 & 41 & 233 & 598 & 1117 & 1071 & 2898 & 1792 & 4022 & 1272 \\
\hline 54 & 0.50 & 36 & 39 & 203 & 553 & 1001 & 929 & 2498 & 1514 & 3749 & 1282 \\
\hline 58 & 0.56 & 22 & 36 & 171 & 505 & 894 & 805 & 2158 & 1282 & 3458 & 1270 \\
\hline 63 & 0.63 & 18 & 32 & 140 & 455 & 796 & 697 & 1866 & 1088 & 3158 & 1240 \\
\hline 67 & 0.70 & 12 & 27 & 112 & 405 & 707 & 603 & 1616 & 925 & 2859 & 1192 \\
\hline 71 & 0.77 & 11 & 23 & 86 & 356 & 625 & 521 & 1400 & 787 & 2565 & 1129 \\
\hline 75 & 0.84 & 8 & 18 & 65 & 310 & 552 & 450 & 1215 & 672 & 2283 & 1055 \\
\hline 80 & 0.92 & 5 & 15 & 48 & 267 & 486 & 389 & 1056 & 573 & 2017 & 973 \\
\hline 84 & 1.00 & 2 & 11 & 34 & 227 & 427 & 335 & 918 & 490 & 1768 & 885 \\
\hline
\end{tabular}




\subsubsection{Cumulative Fatigue Damage Totals}

Having produced an estimated fatigue loading spectrum for an entire year long wind regime, the final step in providing an estimate for the 20 year fatigue life of the Gorman Controls' wind turbine blades is the tabulation of the cumulative damage caused by each stress cycles. At each stress level predicted in the fatigue loading spectrum, the alternating stress, $\sigma_{\mathrm{a}}$, can be calculated using Equation (3.1) since the stress ratio is a known value and each stress level corresponds to a mean stress, $\sigma_{\mathrm{m}}$. Equation (3.3) can then be used to equate this non-zero mean alternating stress to the appropriate zero-mean alternating stress. Having completed this task, the S-N curve derived for the wooden laminate blade can then be used in accordance with Miner's Rule to predict the total progressive damage. Assuming the WEICAN wind profile is predictive of a 20 year average wind regime, the single year cumulative damage totals may simply be multiplied by 20 to achieve the desired total. After completing these calculations, the total fatigue life consumed by the predicted fatigue loading spectrum was determined to be 0.21 or $21 \%$ of the total fatigue life of the blade. The tabulated fatigue damage totals me be seen in Chapter 6, Table 6-2. 


\section{Chapter 4. Experimental Equipment, Methods and Results}

Proper validation of analytical models is a necessary step in any scientific experiment. A prediction for the fatigue life of the Gorman Controls' wind turbine blades was approximated using analytical models and the theory associated with fatigue damage. The next step was to validate this prediction by performing a full scale fatigue life test on the blades by applying an accelerated loading test on two sample blades. In combination with this test, the material properties of the wooden laminate were investigated as well as the stress concentrations due to the wood and glue line interfaces. A validation of the as-build three dimensional blade profile was also completed as a means of certifying the manufacturing process by which the blade was created. A complete description of all experimental equipment and procedures may be found in the following sections.

\subsection{IN-FIELD BLADE ROOT STRAIN MEASUREMENT}

As a first step in the investigation of the fatigue life of the Gorman Controls' wind turbine blades a study of the in-field loading conditions on the blades was attempted. This effort was made in parallel with Professor John Ridgely, Professor Patrick Lemieux and fellow students at Cal Poly State University. A measurement campaign whereby two full Wheatstone bridge strain gage stations attached to the root of two blades was decided upon by the team. This would provide information about the maximum and minimum bending moments on root of each blade during flight. After surveying the literature in regards to wind turbine blade testing, it was decided that any direct wire and slip ring methods of collecting data were going to be too cumbersome and complicated to perform the desired measurements. A simplified wireless data acquisition device (DAQ) was thus manufactured and attached to the rotor center. The device took as input the two full bridge strain gage stations sampled each at $500 \mathrm{~Hz}$ for three seconds and then transmitted these signals via radio to a ground based PC workstation. Unfortunately, during a preliminary test of the complete wind turbine system, a failure occurred which saw the turbine fall from 
the top of the tower. No useful measurement of the in-field loading parameters was obtained as a result.

\subsubsection{Wireless Data Acquisition Device}

A custom made data acquisition controller was fabricated by Professor John Ridgely using a programmable logic controller, bus terminals for the strain gage inputs, a wireless radio card and a custom build circuit board. Programming for the device was performed by students at Cal Poly. The device may be seen in Figure 4-1 as mounted within a PVC support structure and then attached to the wind turbine front rotor support plate.

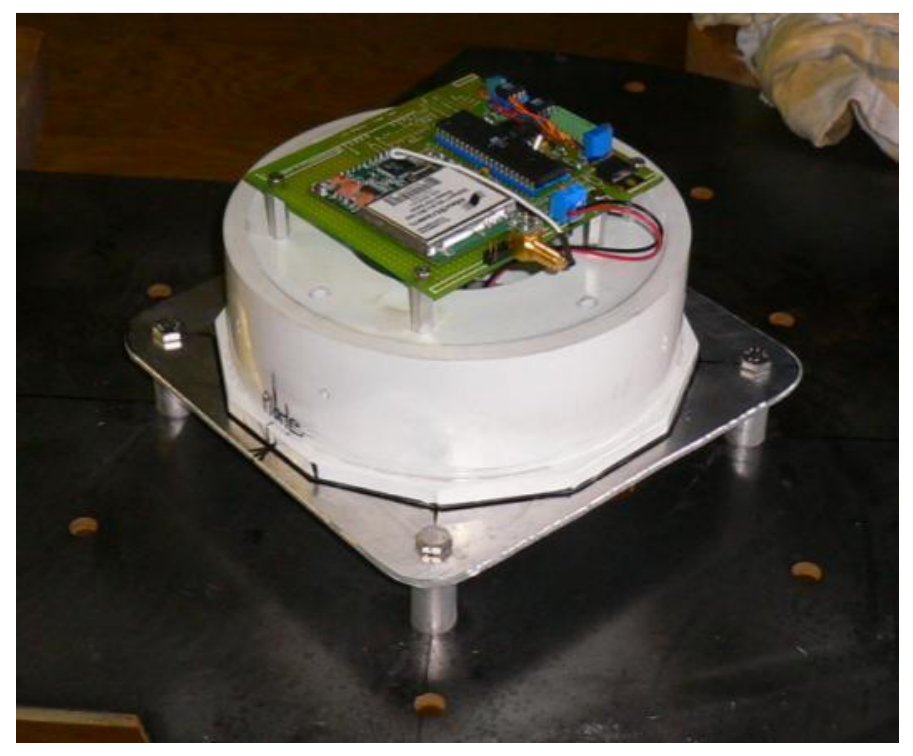

Figure 4-1. Wireless Data Acquisition Device mounted on PVC support frame and rotor center support.

The device was capable of sampling three channels of data at a custom sample rate. It was powered by an on-board 9 volt battery which was stored in the middle of the PVC support frame. A user interface to the module was available using the Hyper-Terminal program within the Windows PC operating environment and a USB cable connected to the device. A user could select the desired sample frequency up to a maximum of $1000 \mathrm{~Hz}$ and could select the number of data points up to a maximum of 2000 points. These setting effectively controlled how much data would be sampled per strain gage channel. The device could also be set to sample at predetermined intervals, switching to a low power mode in 
between these intervals to conserve battery power. Figure 4-2 shows the results of a bench test performed to investigate the first natural frequency of the blades. The sample rate and time span were determined to be too low for this test as one cycle of blade response was gathered by one a few points of data. To avoid signal aliasing, for subsequent tests, the target sample frequency was set to $500 \mathrm{~Hz}$ and the number of data point was set to 2000 .

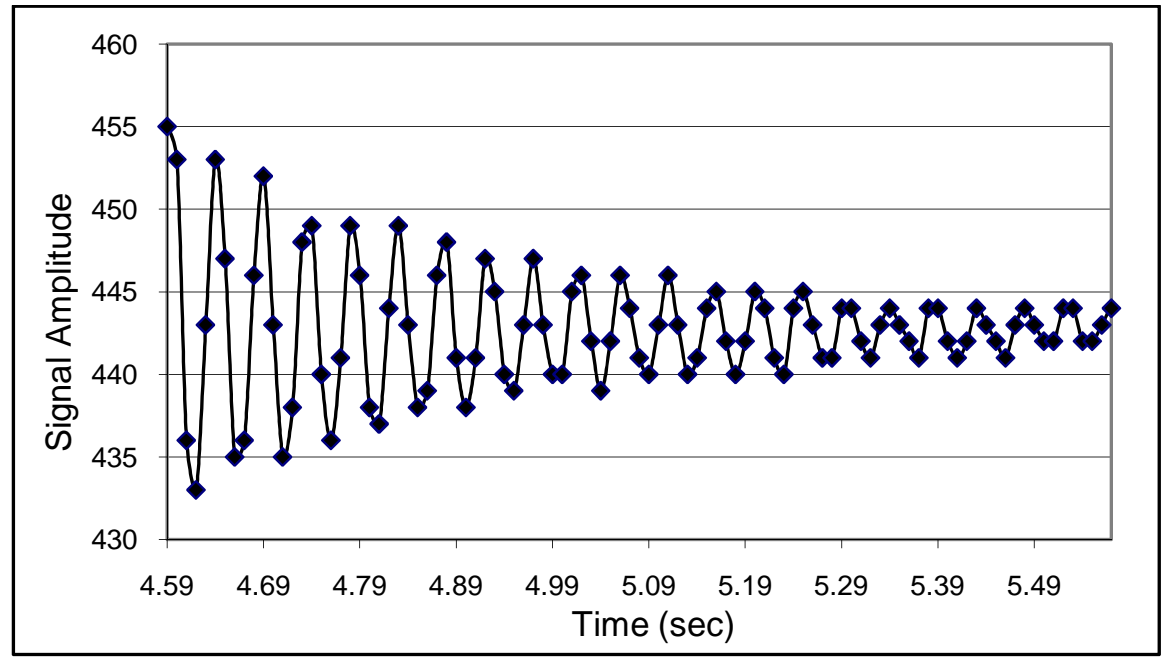

Figure 4-2. Sample output during a bench natural frequency test using the wireless data acquisition device.

\subsubsection{Strain Gage Installation on Blades}

It was decided that only the maximum and minimum bending stresses were to be gathered from the blades of the operating wind turbine. To accomplish this, two full Wheatstone bridge strain gage configurations were mounted to the blade roots of two blades. A Wheatstone bridge is a measurement device created by S. Christie and developed further by $\mathrm{C}$. Wheatstone which is capable of detecting small changes in voltage measured across four balanced legs of a resistive circuit (Dally and Riley 2005). Strain gages were obtained from Vishay, Inc. and a four inch gage length device was selected, model N2A-06-20CBW350. Using methods outlined by Vishay, four gages were bonded to the blades in symmetric locations as would be appropriate for a full bridge sensing circuit; two on the pressure side and two on the suction side of the blade. Figure 4-3 shows two strain gages bonded to the root of a blade with the lead wires attached for configuration within a Wheatstone bridge. An identical pair of gages was bonded to the opposite side of the blade to complete the 
circuit. Reflective metallic tape was used to seal the gages on the blade and protect the sensing wires from the elements.

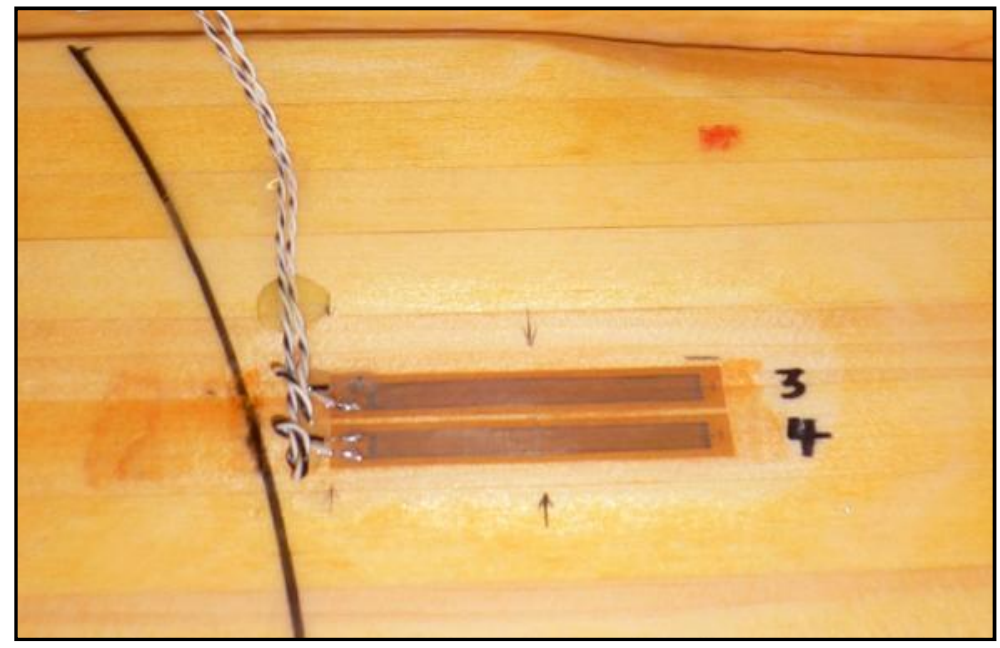

Figure 4-3. Dual strain gages attached to the root of the wind turbine blade with lead wires for Wheatstone bridge attachment.

\subsubsection{Attempted In-Field Blade Load Measurement}

Using the wireless DAQ and the attached strain gages on the blades, the in-field measurement of blade root flapwise bending stresses was attempted. Figure 4-4 shows the complete DAQ system including the sensing gages protected by silver tape as mounted on the wind turbine rotor. Unfortunately, shortly after installation of this device was complete, the wind turbine suffered a yaw bearing failure and fell from the 30 meter tower. The DAQ managed to survive the fall but the entire wind turbine suffered a catastrophic failure. Figure 4-5 shows the wind turbine directly after the fall and as can be seen in the image, the blades were completely destroyed. This failure prompted the author to pursue alternative methods of obtaining the critical blade loading parameters and the numerical model described in Chapter 3 was a direct result of this setback. 


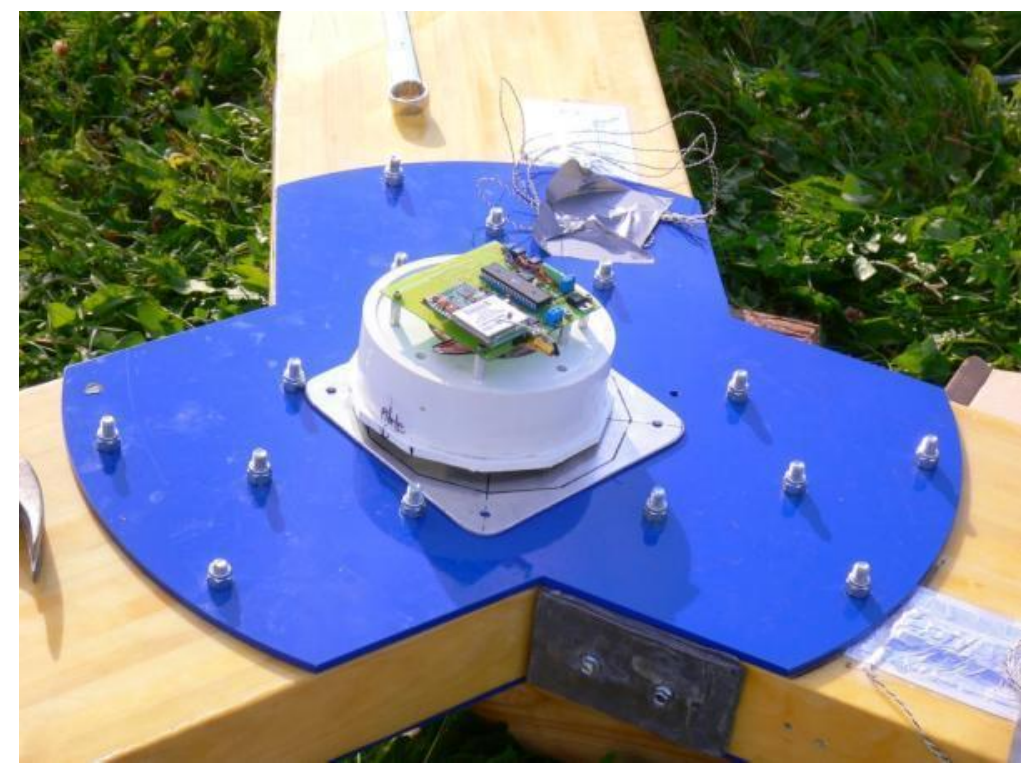

Figure 4-4. Wireless DAQ attached to the rotor center with two Wheatstone strain gage sensing circuits attached to the blade root.

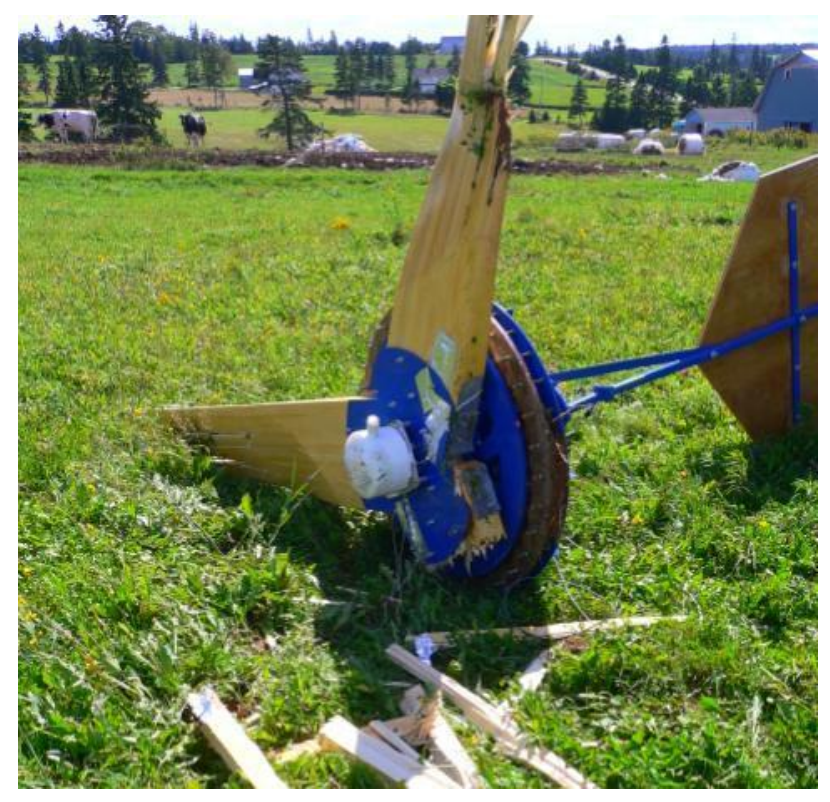

Figure 4-5. Gorman Controls' $10 \mathrm{~kW}$ wind turbine immediately after falling from the 30 meter tower. 


\subsection{MATERIAL PROPERTY TESTING}

In order to correctly predict the response of the wooden laminate blades to typical wind loading, the material properties of the wood are needed. Careful study of appropriate methods for determining these experimental values were considered and the methods outlined by the American Society of Testing and Materials (ASTM) in the D-143 standard were used (ASTM D-143 2002). The roots of all three wooden composite blades from Gorman Controls Ltd. were cut into several small coupons in order to determine Young's Modulus along the grain for the white pine from which the blades were constructed. Several similar specimens were cut in different orientations in order to determine the ultimate strengths in off grain directions as well as the major and minor Poisson's ratios for the material. The material property values were measured using bi-axial strain gages located at the mid section of each tensile coupon. Corresponding stain data was collected using a Micro Measurements PC-1 strain indicator and appropriate material constitutive theory was used to calculate each material property. These values compare well with those published for white pine as provided in the Wood Handbook (Forest Products Laboratory 1999). A simple volume cube of material cut from the blade was also used to measure an approximate density of the composite blade material. The reader is referred to Figure 3-2 for a description of the principal material directions defined for wooden samples by the American Society of Testing and Materials.

\subsubsection{ASTM Testing Methods Used}

Two separate tests were selected to determine the material properties of the wooden laminate used to construct the blades. The following sections briefly describe each method.

\subsubsection{ASTM D-143: Tensile Strength Testing}

This standard outlines a tensile test using a small thin coupon of material in order to measure the Young's Modulus in the fiber direction as well as the Poisson ratio in the 1-2 and 1-3 directions. Small rectangular wooden coupons are to be made which exhibit a length to depth ratio of at least 12:1 with the wood grain being parallel to the length. Biaxial strain gages are to be applied to the middle of the specimens on two orthogonal sides 
with the principal longitudinal axis oriented parallel to the wood fibers. Grip tabs are to be created for mounting the coupons within the vise grips of a loading machine so the coupons are not damaged in compression. The coupons are to be loaded at a rate which will produce failure in less than 2 minutes but should not be reached in less than 10 seconds in order to avoid the effects of adiabatic heating. The strain values measured should be recorded with a time stamp and load level stamp which documents the loading rate. The cross sectional area should be recorded prior to testing so stress values can be calculated using basic $\mathrm{P} / \mathrm{A}$ stress analysis where $\mathrm{P}$ is load and $\mathrm{A}$ is the cross section area. Maximum load at failure should be recorded as well.

\subsubsection{ASTM D-143: Compressive Strength Testing}

This standard outlines a test for determining the ultimate compressive strength of wood coupons. Small rectangular coupons are to be cut which have a length equal to at least 2.5 times the depth dimension. The wood grain is to be parallel to the length of the specimen. Similar grip tabs and loading rates to the tensile test previously mentioned applies to this test as well. Only the cross-head displacement and load at failure of the specimen are to be recorded.

\subsubsection{Wood Coupon Sizing}

Four tensile coupons were cut parallel to the grain direction from each blade root, as well as four compressive coupons in the grain direction and three off-grain compressive coupons were cut as well. Figure 4-6 shows the location within the root of the blade where each sample coupon was cut. Coupons were cut from this section of the blade because the clamping device used for the full scale fatigue life test attached to the blade root at a location 22 inches away from the triangular blade end and it was determined that this region was not need for direct blade testing but could instead be used for material property testing. 


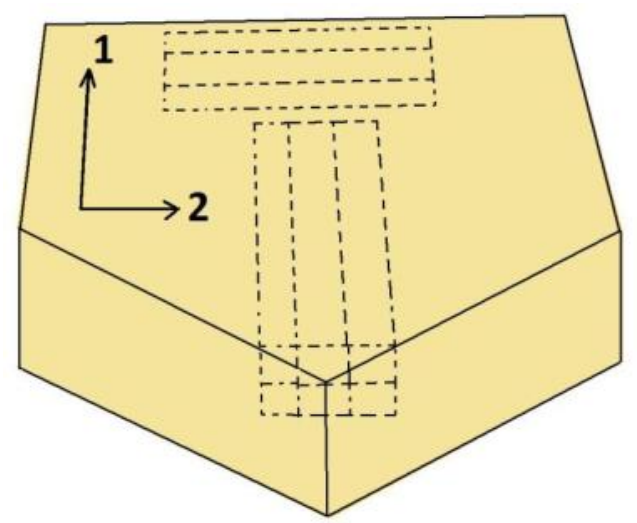

Figure 4-6. Illustration of location within blade root where tensile and compressive wood coupons were cut. 1) is the wood grain direction and 2) is the perpendicular grain direction.

The dimensions for each coupon may be seen in Table 4-1 along with a note regarding the orientation of the grain direction.

Table 4-1. Material property testing coupon dimensions.

\begin{tabular}{|l|l|c|c|c|c|}
\hline \multicolumn{1}{|c|}{ Specimen } & \multicolumn{1}{|c|}{$\begin{array}{c}\text { Grain } \\
\text { Orientation }\end{array}$} & $\begin{array}{c}\text { Length } \\
\text { (in) }\end{array}$ & $\begin{array}{c}\text { Width } \\
\text { (in) }\end{array}$ & $\begin{array}{c}\text { Cross Section } \\
\text { Area }\left(\text { in }^{2}\right)\end{array}$ & $\begin{array}{c}\text { Grip Tab } \\
\text { Size (in) }\end{array}$ \\
\hline Tensile & Parallel & 7 & 0.5 & 0.25 & 0.25 \\
\hline Compression & Parallel & 5 & 0.5 & 0.25 & 0.25 \\
\hline Compression & Perpendicular & 5 & 0.5 & 0.25 & 0.25 \\
\hline
\end{tabular}

\subsubsection{Testing Devices}

Two testing devices were utilized to measure the material properties of the wooden laminate, both manufactured by Instron. An Instron 4400R tensile test machine capable of applying a 1,000 pound vertical tensile load was used to test the tensile strength and Young's Modulus of the wooden laminate. This machine has a fixed bottom grip and moveable top cross head with grip which is mechanically controlled by motors. Figure 4-7a shows a wooden tensile coupon being tested in the machine. The orientation of the strain gages on the sample may be seen in Figure 4-7b. An Instron 8500 tensile and compression test machine was used to test the wooden samples in compression. This machine has a fixed upper head and a hydraulically activated lower cross head capable of 
applying both compression and tensile loads over 20,000 pounds. Both machines have a fixed grip size of $1 / 4$ inch by 1 inch and each wood coupon was cut the fit within these grips.

Strain values during the testing process were measured using a National Instruments LabVIEW virtual instrument (VI) which was created by the faculty and students within the Mechanical Engineering department at Cal Poly. This program was able to store the load, time and strain values from 2 strain gages simultaneously during each test. This data was then used to construct stress-strain diagrams for the coupons and estimate the material properties for the wooden laminate.

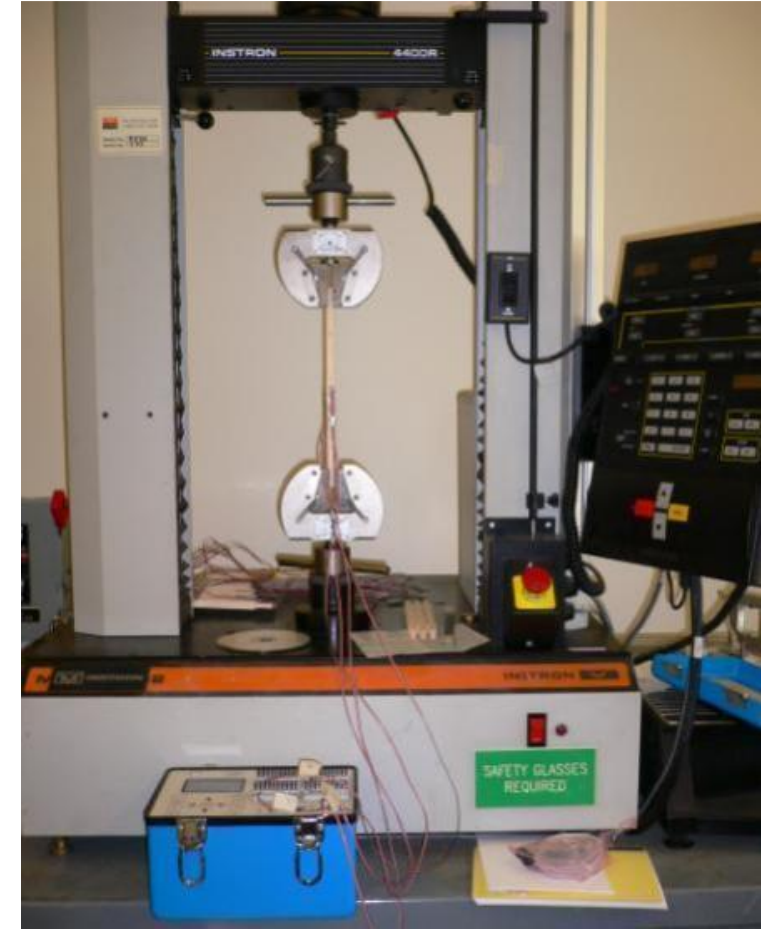

(a)

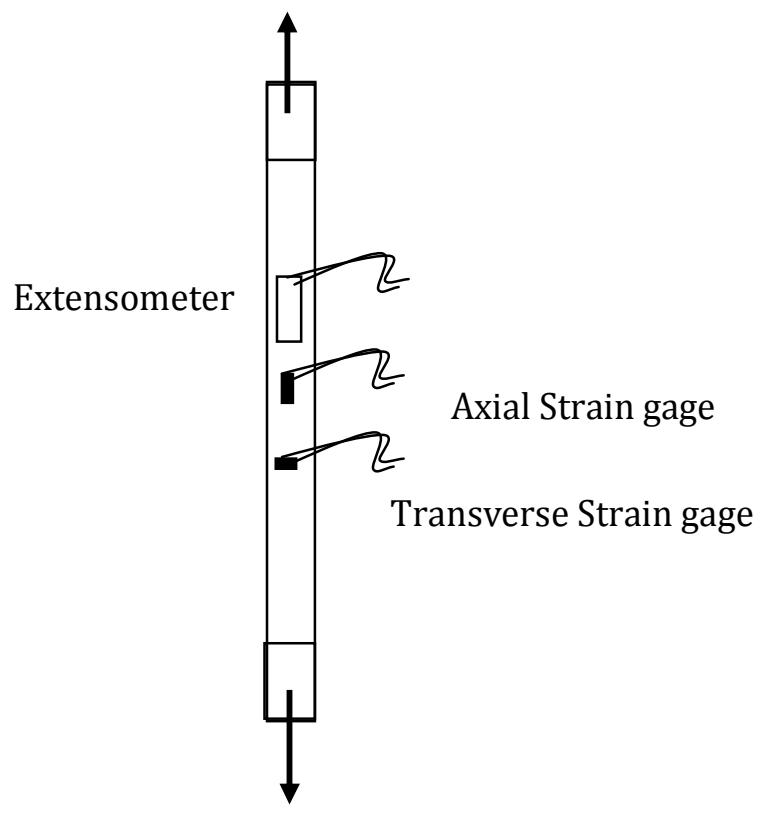

(b)

Figure 4-7. a) The Instron 4400R tensile test machine used to determine the Young's Modulus of the wood. b) The location of strain gages on a sample tensile test coupon.

\subsubsection{Material Property Estimation}

Following the testing procedures outlined in the ATSM D-143 testing specification each wooden coupon was tested to failure. A total of 12 parallel grain tensile tests, 12 parallel grain compression tests and 9 off-grain compression tests were performed. The material 
properties derived from these test were calculated using basic equations from the theory of elasticity and the constitutive equations relating strain and stress. A brief summary of material constitutive theory is presented in the following section. Figure 4-8 shows one of the 12 tensile tests performed to estimate the modulus of elasticity of the wood in the longitudinal direction. Using the equations from the theory of elasticity, the $\mathrm{E}_{1}$ value is simply the slope of the curve generated in a stress versus strain plot.

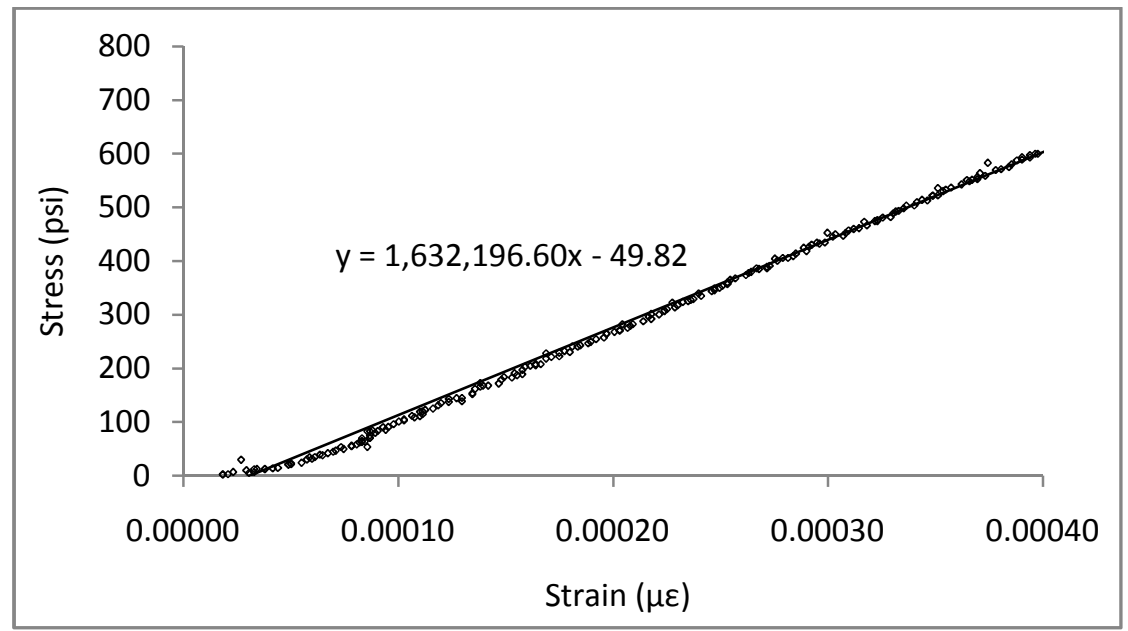

Figure 4-8. Sample test performed to estimate the modulus of elasticity in the longitudinal or fiber direction. Stress is shown as a function of strain.

The Young's Modulus in the 1 or longitudinal direction was found to be 1.62E6 psi and the Poisson's ratio in the 1-2 direction was found to be 0.41 which is higher than typical engineering materials such as steel which is typically 0.3 . The ultimate strength the fiber direction was found to be 5059 psi in tension and 2036 psi in compression. The ultimate strength in compression perpendicular to the fiber direction was found to be $444 \mathrm{psi}$. Table 4-2 shows the results from all the material property tests. A calculation for the lower $95^{\text {th }}$ percentile confidence interval value for each material property is included in the table. As mentioned in Chapter 3, the S-N master curves created by Bond and Ansell were based on this lower confidence interval because it allowed the master curve to reflect a confidence of $95 \%$ because the material properties used to construct the curve also reflected this confidence level. The published values for typical white pine clear grain specimens as defined in the Wood Handbook are listed in Table 4-2 and these values are 
shown to agree well with the experimentally measured ultimate strength values. The experimentally measured modulus of elasticity is shown to be greater than the published value by $12 \%$ and this shows that the laminated wooden structure used by Gorman Controls is slightly stiffer than a clear wood specimen. These results confirm the assertion by Bond and Ansell that the material properties of wooden laminates do not differ greatly when compared with their clear wood counterparts (Bond and Ansell 1998).

Table 4-2. Material property values measured for the wood laminate blade material.

\begin{tabular}{|c|c|c|c|c|c|c|c|}
\hline Property & $\begin{array}{c}\text { Number } \\
\text { of Tests }\end{array}$ & $\begin{array}{c}\text { Mean } \\
\text { Value }\end{array}$ & Units & $\begin{array}{c}\text { Standard } \\
\text { Deviation }\end{array}$ & $\begin{array}{c}\text { Lower } \\
95 \% \mathrm{Cl} \\
\text { Value }\end{array}$ & $\begin{array}{c}\text { Published } \\
\text { Value }\end{array}$ & \% Difference \\
\hline $\mathrm{E}_{1}$ & 12 & $1.63 \mathrm{E}+06$ & $\mathrm{psi}$ & 25645 & $1.62 \mathrm{E}+06$ & $1.46 \mathrm{E}+06$ & $11.97 \%$ \\
\hline $\mathrm{v}_{12}$ & 12 & 0.41 & - & 0.041 & 0.39 & - & - \\
\hline $\mathrm{Su}_{\mathrm{T}, 1}$ & 12 & 5059 & $\mathrm{psi}$ & 54.86 & 5028 & 5040 & $0.38 \%$ \\
\hline $\mathrm{Su}_{\mathrm{C}, 1}$ & 9 & 2036 & $\mathrm{psi}$ & 77.05 & 1992 & 2100 & $-3.07 \%$ \\
\hline $\mathrm{Su}_{\mathrm{C}, 2}$ & 9 & 444 & $\mathrm{psi}$ & 40.63 & 418 & 470 & $-5.44 \%$ \\
\hline
\end{tabular}

\subsubsection{Stress-Strain Relationships for Transversely Isotropic Materials}

A wooden structure such as the blades of the Gorman Controls' wind turbine may be considered a transversely isotropic material since all of the fibers of the wood are oriented in the longitudinal direction. The effective elastic constants are therefore isotropic on the plane with its outer normal in the 1 or longitudinal direction. Readers are referred to Figure 3-2 for a definition of the fiber and principal axis orientations. This isotropy results in a reduction in the number of independent constants which must be measured to properly identify the stiffness matrix for the material. Readers are referred to the book Experimental Stress Analysis for a complete explanation (Dally and Riley 2005). If a plane stress is introduced to the specimen such as pure bending, it can be shown that only four independent elastic constants need be measured to fully write the stress-strain relations. These constants are: $\mathrm{E}_{1}$, the Young's Modulus in the 1 direction, $\mathrm{E}_{2}$, the Young's Modulus in the 2 direction, $\mathrm{G}_{12}$, the shear modulus in the 1-2 plane, and $v_{12}$, the Poisson ratio in the 1-2 
plane. It can be shown that for a transversely isotropic composite under a plane stress where $\sigma_{3}=\tau_{23}=\tau_{32}=0$ and $E_{2}=E_{3}$ the stress-strain relationships reduce to the following:

$$
\begin{gathered}
\sigma_{1}=\frac{E_{1}}{1-v_{12} v_{21}}\left[\varepsilon_{1}+v_{21} \varepsilon_{2}\right] \\
\sigma_{2}=\frac{E_{2}}{1-v_{12} v_{21}}\left[v_{12} \varepsilon_{1}+\varepsilon_{2}\right] \\
\tau_{12}=G_{12} \gamma_{12}
\end{gathered}
$$

Furthermore, if $v_{21}$ is shown to approach zero which occurs because the material is far stiffer in the fiber direction than the transverse matrix direction, then Equations (4.1)and (4.2) reduce to the following equations:

$$
\begin{gathered}
\sigma_{1}=E_{1} \varepsilon_{1} \\
\sigma_{2}=E_{2}\left[v_{12} \varepsilon_{1}+\varepsilon_{2}\right] \\
\tau_{12}=G_{12} \gamma_{12}
\end{gathered}
$$

It has been shown by the Forest Products Laboratory of the US that the Poisson's ratio in the 2-1 plane, the one associated with a load applied in the transverse fiber direction, $v_{21}$, is close to zero for most wood species. The publication provided by the laboratory, The Wood Handbook, provides a summary of the Poisson ratios for various wood species and the Poisson ratio associated with this type of loading for eastern white pine is listed as 0.008 which is less than $2 \%$ of the Poisson ratio in the 1-2 direction. Furthermore, the handbook offers the suggestion of quantifying the material behavior of wooden members as transversely isotropic and using only the fiber direction modulus, $\mathrm{E}_{1}$, when all of the five independent material constants are not readily available. Following this recommendation, in order to calculate the Young's Modulus in the fiber direction, only the stress, $\sigma_{1}$, and strain, $\varepsilon_{1}$, in that direction need to be measured. Similarly, to find the Poisson ratio $v_{12}$, only the strain in the one and two directions need be measured along with the modulus in the two direction, $\mathrm{E}_{2}$. It was assumed that the wooden laminate used for the blades of the Master's Thesis 
Gorman Controls' wind turbine was a transversely isotropic material and would be well defined by the equations presented above. This assumption was validated by testing the wooden laminate in pure bending and observing that the stress in the transverse direction was less than $5 \%$ of the stress in the fiber direction. This experimentally tested value lends credibility to the assumption of assuming that $\sigma_{1}$ is the only non-zero stress term and the Poisson ratio $v_{12}$ may be found using the following equation which is found by assuming $\sigma_{2}$ $=0$ and reducing Equation (4.5) to:

$$
\varepsilon_{2}=-v_{12} \varepsilon_{1}
$$

\subsection{THREE DIMENSIONAL BLADE PROFILE VERIFICATION STUDY}

The typical mechanical response of each wooden blade to certain wind velocities was calculated using a numerical code based on an ideal profile model of the blade. While this model was based on the construction geometry, variability in the manufacturing processes may have unknown effects on the mechanical properties of the blades. Furthermore, variability in the profile of each blade is unknown and may significantly affect the aerodynamic performance of each individual blade. To address this issue, a study was conducted to quantify the error associated with manufacturing by creating a three dimensional profile of three blades, as-built from the manufacturer. A laser part scanner was used to capture small point cloud data areas along a blade and then consecutive scans were made in order to capture the entire blade profile. The scans were stitched together using a program called GeoMagic and eventually a 3D model of each blade was completed which could be analyzed using SolidWorks, a commercial modeling program. The profiles were then compared to the theoretical design profiles and percent deviations between the two models were calculated. Figure 4-9 shows the laser part scanner during one of the many scans with the PC workstation used to store the scanned data and perform the post processing necessary to complete a model.

\subsubsection{Blade Scanning Procedure}


To begin the process of scanning all three blades provided by Gorman Controls, a LDI PS 4000 laser part scanner was obtained. This device was borrowed from the Cal Poly Industrial Engineering Department. The LDI laser part scanner worked by sweeping a precise linear laser beam across a part and then sampled the reflectivity of the laser off of the object. The individual photons emitted by the laser would reflect off the part and then pass through a detector on the device. By calculating the time lapse between emission and detection of the photons, a distance from the emitter to the part could be calculated. Each photon would be assigned a specific distance from the emitter which then represented a point on the surface of the scanned part. If a scanned part contained uneven surfaces, the path of the photon would be altered and this alteration was directly linked to a time lapse and therefore a distance. In this way, a complicated surface could be accurately measured and stored as a collection of points with associated distances from the emitter. A collection of these measured data points, referred to as a point cloud, was generated every time a scanning sweep was completed. The effective measurement window provided by the scanner was only 18 inches by 18 inches so multiple scans were completed to capture the entire 122 inch blade surface, front and back. Ultimately, over 80 scans were performed to completely define the entire surface of one blade. This time consuming process was completed three times to model each blade sent to Cal Poly by Gorman Controls. Figure 4-10 shows blade B during the scanning process. The blade is painted white because the reflectivity of the glossy wood surface introduced significant errors in the scanning process and a white surface was determined to be the best for scanning. White beads were also glued to the surface and acted as reference points during the point cloud merging process. 


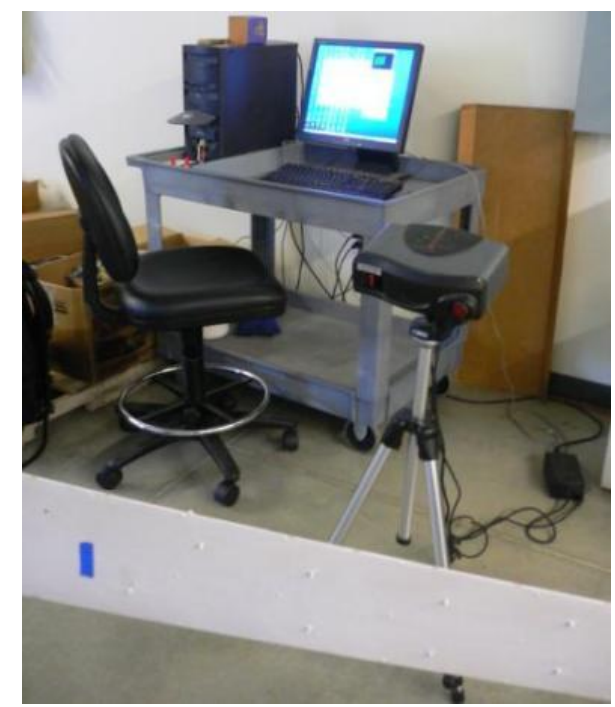

Figure 4-9. The LDI PS 4000 laser part scanner shown during a scan of blade C. The associated computer workstation can be seen in the background.

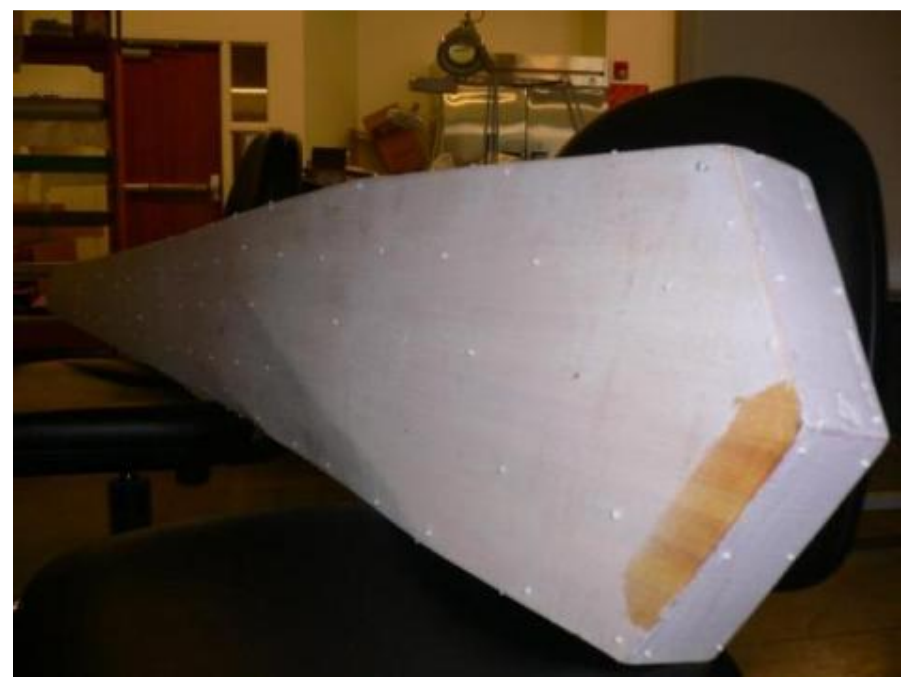

Figure 4-10. Blade B shown during the scanning process with white beads glued to the surface as reference points.

\subsubsection{Merging Point Cloud Data}

Once the entire surface of a blade had been scanned, the point cloud data needed to be merged into one single model. This process proved to be equally time consuming since each point cloud of data was merged together by manually selecting common points 
between two point clouds and then visually inspecting the merged result to ensure accuracy of the model. Further analysis was needed once this task was completed due to computer errors which added very small arbitrary holes in the merged surfaces. The computer software, GeoMagic, used to create the merged point cloud model had good editing tools which allowed these small holes to be repaired without damaging the overall surface of the blade. Figure 4-11 shows a series of holes which were repaired using these tools. Once every point cloud was merged together and all holes were removed, the complete blade was converted into a surface and export of this surface into a solid modeling program was allowed via conversion of the model into an IGES file. This allowed easy import of the competed model into a solid modeling program such as SolidWorks.

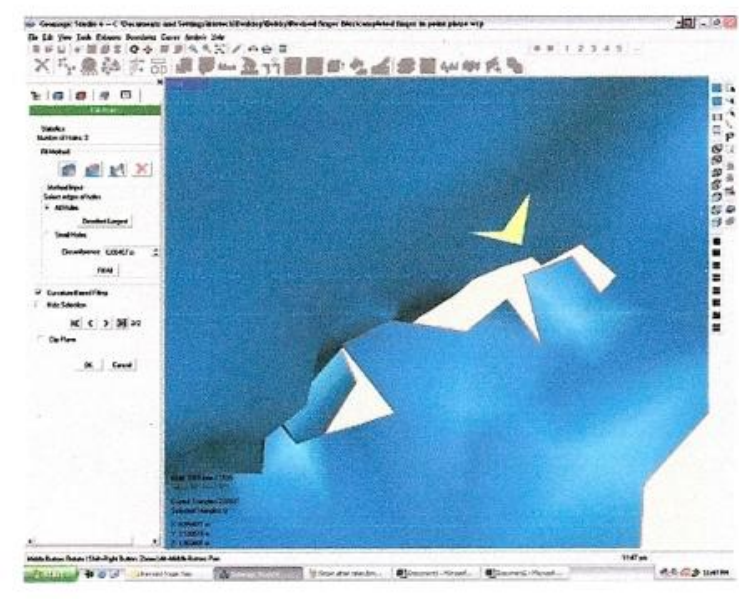

(a)

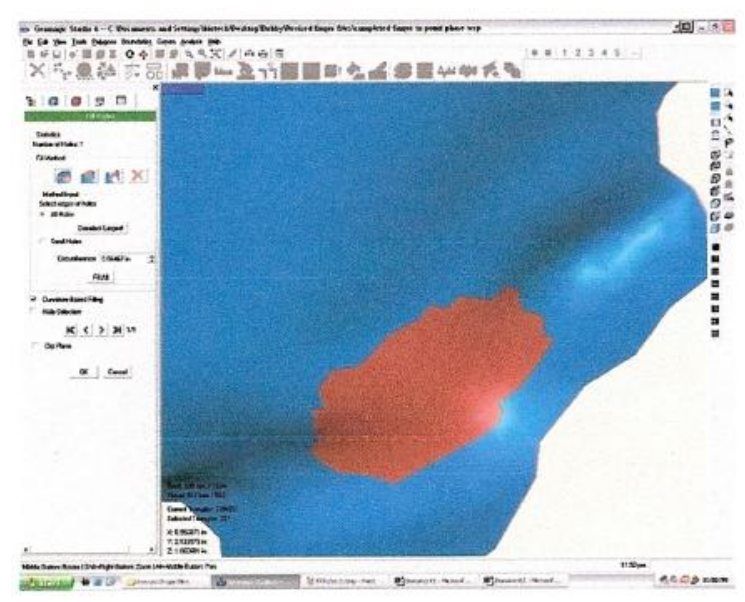

(b)

Figure 4-11. Illustration of the surface and hole repair operations performed when merging the many point cloud data files for each blade. a) Holes before repair, b) repaired surface.

The verification study comparing the as-built blades to the designed profile was completed using SolidWorks. Figure 4-12 shows the completed 3D model of blade A as viewed within SolidWorks. 


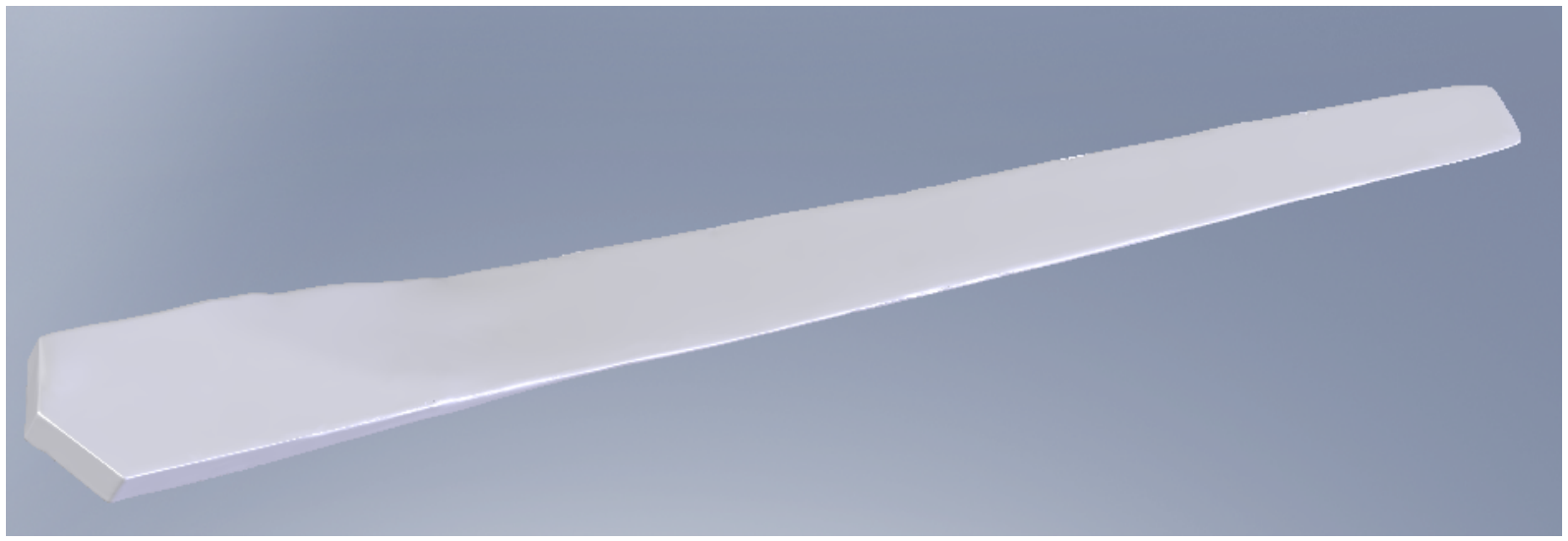

Figure 4-12. The completed 3D scanned model of blade A, as viewed from within the CAD software package SolidWorks.

\subsubsection{Blade Profile Comparisons and 3D Model Verification}

The process variability and model validation was studied by comparing 2D profiles generated a set intervals along each blade. A 2D profile for an ideal Clark Y airfoil profile was created based on dimensions given by the manufacturer and the 3D models of each blade were sectioned and corresponding 2D profiles were created. Ultimately, the blade profile validation study was completed in two parts: the 2D scanned profiles were first compared against an ideal Clark Y airfoil to estimate the error between the real and ideal profiles. And second, each profile was compared to each other to assess the variability in the manufacturing process between each blade. It was decided that the entire length of the blade would not be studied since the important sections of the blade with respect to process variability were the airfoil sections from mid-span to the tip of the blade. Since almost all significant aerodynamic loading originates at these surfaces it was decided that profiles created at stations staring at 60 inches from the blade root outward at 10 inch intervals were to be studied. The rectangular root section of the blade was considered unimportant from a profile validation standpoint since variability in the region of the blade would not have a significant effect on aerodynamic performance. 


\subsubsection{Comparison with Clark Y Airfoil}

All three blades were sectioned into six matching profiles corresponding to radial profile positions of 60 inches through 110 inches at 10 inch intervals as measured from the root of the blade. Figure 4-13 shows the six profiles which were created by sectioning the 3D model of blade B. Similar profiles were created for blades A and C and may be seen in Appendix B. An ideal Clark Y profile was then generated using a data set provided by the manufacturer. This ideal Clark Y profile was then scaled to have a chord length of one. This allowed this theoretical Clark Y profile to be matched to the as-built profiles generated by sectioning the 3D blades by simply scaling the chord length by an appropriate number. The ideal chord length for each profile station was determined based on the theoretical design of the blades. This information was provided by the manufacturer in the form of drawings showing the dimensions of the ideal blade shape. These drawings may be seen in Appendix B. This process of matching the ideal to the as-built profiles was completed for each of the 18 profiles generated, one for each of the six profiles generated per blade.

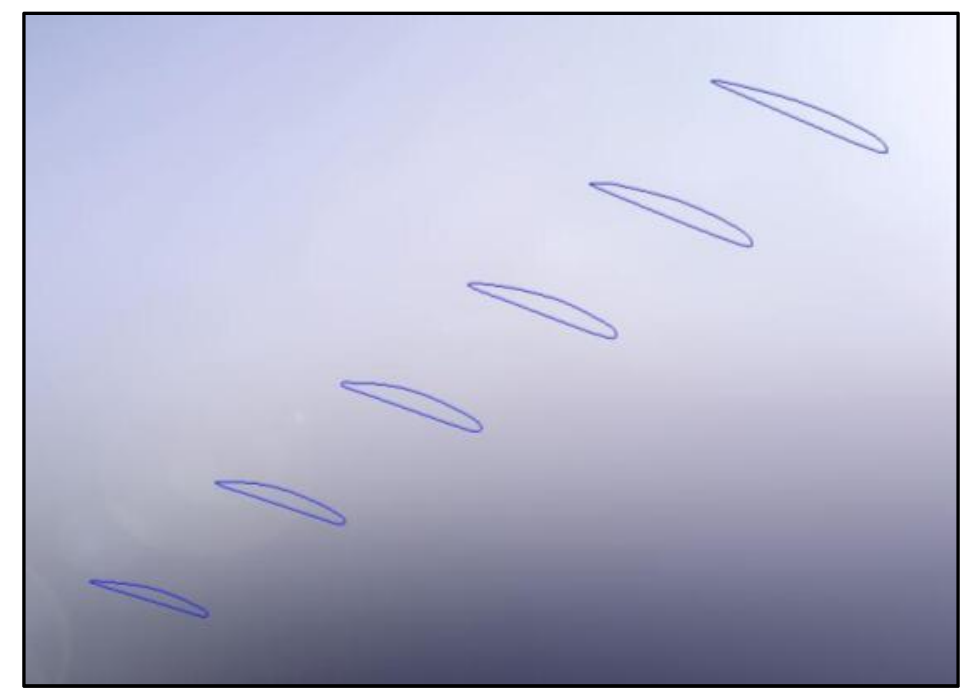

Figure 4-13. Six 2D profiles generated by sectioning the 3D model of blade B.

Measurement of the chord length and ten section thickness values for every profile were performed using the analysis tools within SolidWorks. Figure 4-14 shows the scaled Clark Y profile and the as-built profile for the $2 \mathrm{D}$ profile generate for blade $\mathrm{C}$ at a radial location 
of 90 inches. The overlap between the two profiles can be seen as well as the dashed lines showing the chord and section thickness measurement locations. This analysis was performed for every profile and the complete tabulation of all section thickness and chord lengths may be seen in Appendix B.

After completing this analysis, it was determined that that chord length variation between the as-built profiles and the ideal Clark Y profiles were in close agreement with one another. Comparison of the cross sectional areas of the as-built profiles did not match well, however, with the designed Clark Y areas. The percent difference calculations between these values may be seen in Table 4-3. Figures displaying all of these relationships may be seen in Appendix B.

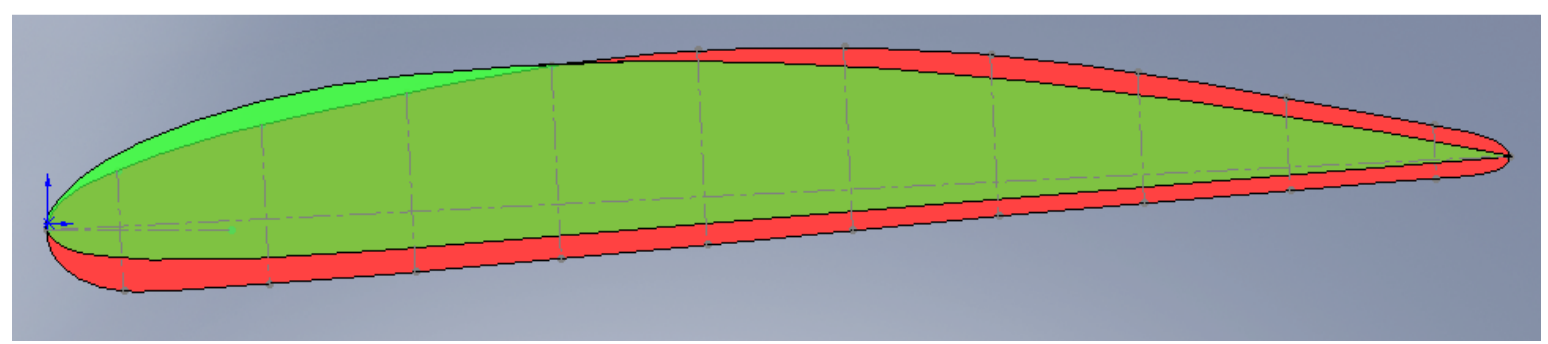

Figure 4-14. Example of the profile matching process for profile of blade number $A$ at a radial location of 90 inches with a chord length of 8.76 inches. Clark Y profile is shown in green and the as-built profile is shown in red. 
Table 4-3. Tabulated values for comparison between the as-built and ideal Clark Y sections.

\begin{tabular}{|c|c|c|c|c|c|c|}
\hline \multicolumn{7}{|c|}{ Blade A } \\
\hline $\begin{array}{c}\text { Clark Y } \\
\text { Chord } \\
\text { (in) }\end{array}$ & $\begin{array}{c}\text { Section } \\
\text { Location (in) }\end{array}$ & $\begin{array}{c}\text { Measured } \\
\text { Chord (in) }\end{array}$ & $\begin{array}{c}\text { Percent } \\
\text { Difference }\end{array}$ & $\begin{array}{c}\text { Clark Y } \\
\text { Area (in }{ }^{2} \text { ) }\end{array}$ & $\begin{array}{c}\text { Measured } \\
\left.\text { Area (in }{ }^{2}\right)\end{array}$ & $\begin{array}{c}\text { Percent } \\
\text { Difference }\end{array}$ \\
\hline 10.67 & 60 & 10.87 & 1.83 & 9.57 & 8.74 & -9.50 \\
\hline 9.95 & 70 & 10.03 & 0.77 & 8.14 & 9.00 & 9.56 \\
\hline 9.24 & 80 & 9.19 & -0.54 & 6.83 & 8.07 & 15.37 \\
\hline 8.52 & 90 & 8.74 & 2.52 & 6.18 & 7.43 & 16.82 \\
\hline 7.80 & 100 & 7.99 & 2.43 & 5.17 & 5.72 & 9.62 \\
\hline 7.08 & 110 & 7.38 & 4.12 & 4.41 & 3.90 & -13.08 \\
\hline
\end{tabular}

\begin{tabular}{|c|c|c|c|c|c|c|}
\hline \multicolumn{7}{|c|}{ Blade B } \\
\hline $\begin{array}{c}\text { Clark Y } \\
\text { Chord } \\
\text { (in) }\end{array}$ & $\begin{array}{c}\text { Section } \\
\text { Location (in) }\end{array}$ & $\begin{array}{c}\text { Measured } \\
\text { Chord (in) }\end{array}$ & $\begin{array}{c}\text { Percent } \\
\text { Difference }\end{array}$ & $\begin{array}{c}\text { Clark Y } \\
\text { Area (in }{ }^{2} \text { ) }\end{array}$ & $\begin{array}{c}\text { Measured } \\
\left.\text { Area (in }{ }^{2}\right)\end{array}$ & $\begin{array}{c}\text { Percent } \\
\text { Difference }\end{array}$ \\
\hline 10.60 & 60 & 10.71 & 0.97 & 9.28 & 8.56 & -8.46 \\
\hline 9.88 & 70 & 9.91 & 0.26 & 7.89 & 8.84 & 10.74 \\
\hline 9.16 & 80 & 9.18 & 0.12 & 6.60 & 8.12 & 18.74 \\
\hline 8.44 & 90 & 8.56 & 1.37 & 5.94 & 7.08 & 15.99 \\
\hline 7.73 & 100 & 7.72 & -0.09 & 4.95 & 5.33 & 7.10 \\
\hline 7.01 & 110 & 7.20 & 2.73 & 4.20 & 3.75 & -12.19 \\
\hline
\end{tabular}

\begin{tabular}{|c|c|c|c|c|c|c|}
\hline \multicolumn{7}{|c|}{ Blade C } \\
\hline $\begin{array}{l}\text { Clark Y } \\
\text { Chord } \\
\text { (in) }\end{array}$ & $\begin{array}{c}\text { Section } \\
\text { Location (in) }\end{array}$ & $\begin{array}{l}\text { Measured } \\
\text { Chord (in) }\end{array}$ & $\begin{array}{c}\text { Percent } \\
\text { Difference }\end{array}$ & $\begin{array}{c}\text { Clark Y } \\
\text { Area }\left(\mathrm{in}^{2}\right)\end{array}$ & $\begin{array}{l}\text { Measured } \\
\text { Area }\left(\mathrm{in}^{2}\right)\end{array}$ & $\begin{array}{c}\text { Percent } \\
\text { Difference }\end{array}$ \\
\hline 10.60 & 60 & 10.46 & -1.39 & 9.72 & 8.84 & -9.96 \\
\hline 9.88 & 70 & 9.88 & -0.03 & 8.24 & 8.94 & 7.77 \\
\hline 9.16 & 80 & 9.25 & 0.91 & 6.93 & 8.09 & 14.32 \\
\hline 8.44 & 90 & 8.43 & -0.23 & 6.22 & 7.57 & 17.84 \\
\hline 7.73 & 100 & 7.63 & -1.20 & 5.34 & 5.77 & 7.58 \\
\hline 7.01 & 110 & 7.21 & 2.81 & 4.43 & 3.95 & -12.20 \\
\hline
\end{tabular}

\subsubsection{Section Profile Variation}

In parallel with the comparison between the Clark Y profile and the as-built profiles, each section profile at a particular radial station was compared to one another. This was performed in order to measure the amount of process variation that is created during the manufacturing of the blades. Three values were compared for every section: the section thicknesses, the chord length and the cross sectional areas. It was shown that the section thicknesses between each blade varied only slightly and the percent difference was measured to be less than $10 \%$ for all but two thickness values. Furthermore, both the 
chord length and cross sectional areas were shown to have less than $10 \%$ variation as well. The complete list of tabulated values may be seen in Table 4-4.

Table 4-4. Results of comparing blade section profiles for blades A,B and C.

\begin{tabular}{|c|c|c|c|c|c|c|}
\hline \multicolumn{7}{|c|}{ Section thickness at 60 inches } \\
\hline \% Chord & Blade A & Blade B & Blade C & A \& B & A \& C & B \& C \\
\hline 5 & 0.75 & 0.73 & 0.76 & $1.7 \%$ & $-2.4 \%$ & $-4.2 \%$ \\
\hline 15 & 1.00 & 0.98 & 1.01 & $1.4 \%$ & $-1.1 \%$ & $-2.5 \%$ \\
\hline 25 & 1.09 & 1.08 & 1.10 & $1.0 \%$ & $-1.1 \%$ & $-2.1 \%$ \\
\hline 35 & 1.10 & 1.10 & 1.12 & $0.5 \%$ & $-1.3 \%$ & $-1.8 \%$ \\
\hline 45 & 1.06 & 1.06 & 1.07 & $0.2 \%$ & $-1.3 \%$ & $-1.5 \%$ \\
\hline 55 & 0.96 & 0.96 & 0.98 & $-0.2 \%$ & $-1.9 \%$ & $-1.7 \%$ \\
\hline 65 & 0.82 & 0.82 & 0.83 & $-0.7 \%$ & $-1.5 \%$ & $-0.7 \%$ \\
\hline 75 & 0.66 & 0.67 & 0.67 & $-1.5 \%$ & $-1.4 \%$ & $0.2 \%$ \\
\hline 85 & 0.47 & 0.47 & 0.47 & $0.4 \%$ & $-0.2 \%$ & $-0.6 \%$ \\
\hline 95 & 0.22 & 0.17 & 0.25 & $25.4 \%$ & $-9.8 \%$ & $-47.3 \%$ \\
\hline
\end{tabular}

\begin{tabular}{|c|c|c|c|c|c|c|}
\hline \multicolumn{7}{|c|}{ Cross Sectional Area $\left(\mathrm{in}^{2}\right)$} \\
\hline Position & Blade A & Blade B & Blade C & A \& B & A \& C & B \& C \\
\hline 60 & 8.74 & 8.56 & 8.84 & $2.1 \%$ & $-1.1 \%$ & $-3.2 \%$ \\
\hline 70 & 9.00 & 8.84 & 8.94 & $1.8 \%$ & $0.7 \%$ & $-1.1 \%$ \\
\hline 80 & 8.07 & 8.12 & 8.09 & $-0.7 \%$ & $-0.2 \%$ & $0.5 \%$ \\
\hline 90 & 7.43 & 7.08 & 7.57 & $4.8 \%$ & $-1.9 \%$ & $-7.0 \%$ \\
\hline 100 & 5.72 & 5.33 & 5.77 & $6.8 \%$ & $-0.9 \%$ & $-8.3 \%$ \\
\hline 110 & 3.90 & 3.75 & 3.95 & $3.9 \%$ & $-1.3 \%$ & $-5.4 \%$ \\
\hline
\end{tabular}

\begin{tabular}{|r|r|r|r|r|r|r|}
\hline \multicolumn{7}{|c|}{ Chord Length (in) } \\
\hline Position & Blade A & Blade B & Blade C & A \& B & A \& C & B \& C \\
\hline 60 & 10.87 & 10.71 & 10.46 & $1.54 \%$ & $3.83 \%$ & $2.33 \%$ \\
\hline 70 & 10.03 & 9.91 & 9.88 & $1.24 \%$ & $1.53 \%$ & $0.29 \%$ \\
\hline 80 & 9.19 & 9.18 & 9.25 & $0.12 \%$ & $-0.67 \%$ & $-0.80 \%$ \\
\hline 90 & 8.74 & 8.56 & 8.43 & $2.00 \%$ & $3.57 \%$ & $1.60 \%$ \\
\hline 100 & 7.99 & 7.72 & 7.63 & $3.42 \%$ & $4.48 \%$ & $1.10 \%$ \\
\hline 110 & 7.38 & 7.20 & 7.21 & $2.42 \%$ & $2.34 \%$ & $-0.08 \%$ \\
\hline
\end{tabular}

\subsection{FULL SCALE BLADE TESTS}

The main objective of this project was the complete documentation of the mechanical fatigue properties, specifically an accurate estimate of the fatigue life in number of cycles, for the laminated wooden wind turbine blades. In order to accomplish this objective, a full scale test was created which subjected each blade to an oscillating bending force in the 
flap-wise, or out of plane direction. The test fixture applied a forced vibration to the blade via a clamped pivot located at the root of the blade. The blade was then allowed to vibrate freely as a cantilevered beam. The frequency of the oscillation was controlled by varying the speed of an induction motor attached to the pivot clamp with a crank disk and connecting rod. The amplitude of the vibration was controlled by adjusting the offset on the crank disk. Each blade was driven at or near the first fundamental frequency for the system, thus taking advantage of the harmonic response of the blade which served to further increased the amplitude of the oscillation. The corresponding amount of tip deflection from horizontal due to this induced vibration was recorded. Strain gages were attached to the root of the blade in half bridge configurations to monitor the amplitude of the induced alternating strains and thus the alternating stresses at this location. A Halleffect sensor and cycle counter mounted near the tip of the blade recorded the cumulative number of cycles during each test by sensing a small magnet attached to the tip. By comparing the alternating stress levels and number of cycles to the fatigue limit curves previously derived, a quantitative estimate of the progressive damage was made. Then, by comparing the progressive damage totals from each test to the derived damage totals found using the rainflow cycle counting method described in Chapter 3 , an accurate estimate of the total fatigue life was made. This wood blade design was found to have a predicted fatigue life greater than 20 years, so far as mechanical fatigue alone is considered. Actual in-field conditions may cause accelerated degradation beyond what is tested and qualified here.

\subsubsection{Mechanical Test Fixture Components}

This section will describe the mechanical attributes of the test fixture used to oscillate the blade during the accelerated fatigue life test. Unless otherwise stated, all components were fabricated by the author using the facilities at Cal Poly State University, San Luis Obispo.

\subsubsection{Steel Clamping Fixture}

As stated in the introduction, the main objective for the vibration fixture was to oscillate the blade at a user defined frequency. The fixture needed to accomplish this task without 
significant deflection since this would change the harmonic response for the entire system. It was therefore decided that a rigid steel clamping device would be designed which would attach to the blade at the root and pivot about a fixed axis at this location. The attachment point to the blade root was designed to simulate the same attachment found on the Gorman Controls' wind turbine so the reactions developed at this location would be similar to those during normal operation of the wind turbine. The axis of rotation provided by this fixture was designed to be very rigid and a 0.75 inch steel rod in combination with rigid steel supports on both the clamp and base plate were used. The driving arm of the fixture was selected to be a quarter inch wall thickness square tube to provide a very stiff attachment to the crank are and motor. The entire pivot clamp was constructed using quarter inch steel plates, custom fabricated and welded by the author. Additional welding was performed by Jim Gerhardt after a fatigue crack had developed at the back of this clamp and additional plates were added to correct this failure. Figure 4-15 shows the designed clamping fixture within the CAD software SolidWorks. The green plates show the original design as built by the author and the gray plates we added to the fixture after the fatigue crack had developed. Shop drawings providing dimensions and material callouts for this fixture may be seen in Chapter 6. 


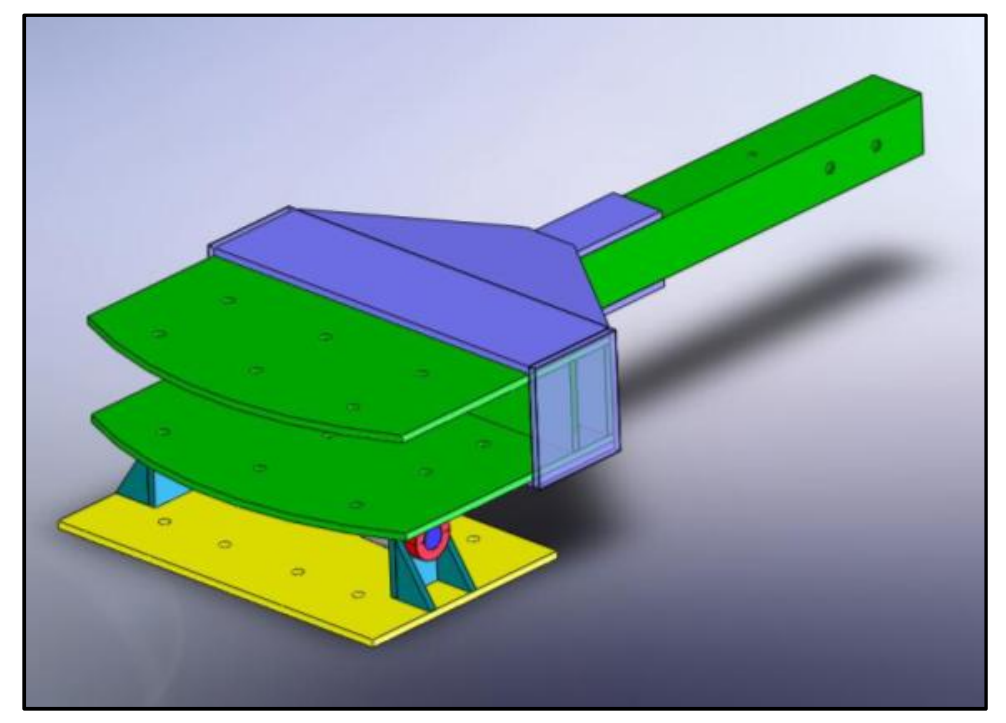

Figure 4-15. The vibration test clamping fixture as seen within SolidWorks. The blade attaches between the flat green plates.

\subsubsection{Support Fame}

The support structure for the vibration test fixture was designed to be a very rigid structure which would support the vibrating blade without significant deflection. To accomplish this task, preliminary hand calculations were performed using a basic design for the support frame and it was shown that using quarter inch steel plates, supported by additional webs and gussets, would create a very rigid structure which would not deflect more than 0.050 inches during loading. The overall design of the structure resembles a small chair with diagonal supports on the back. The blade clamping fixture attaches to the seat of the structure via 3/8 inch bolts and the driving arm passes through the vertical plate on the back of the fixture. Figure 4-16 shows the complete vibration fixture including the blade clamp, motor and crank arm. The support frame is shown in gray. 


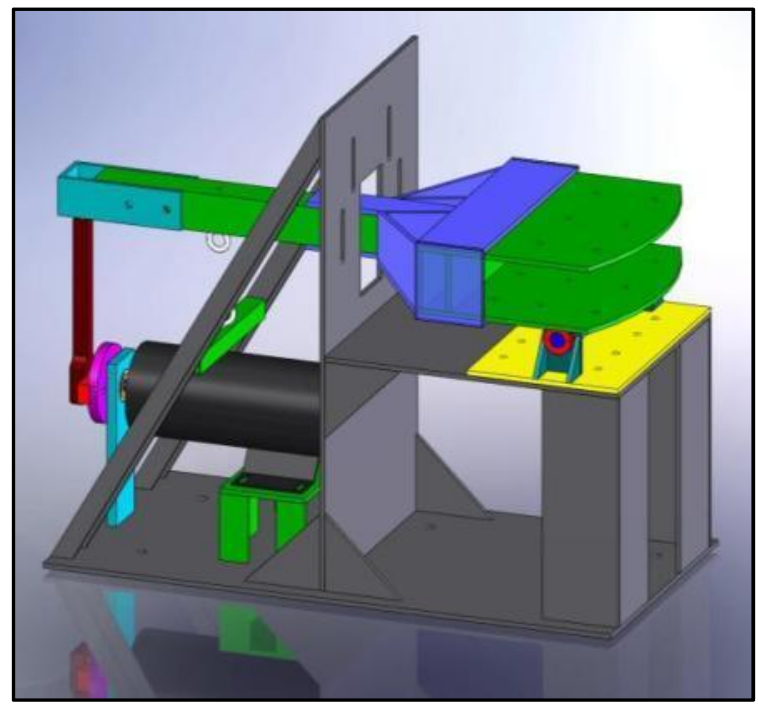

(a)

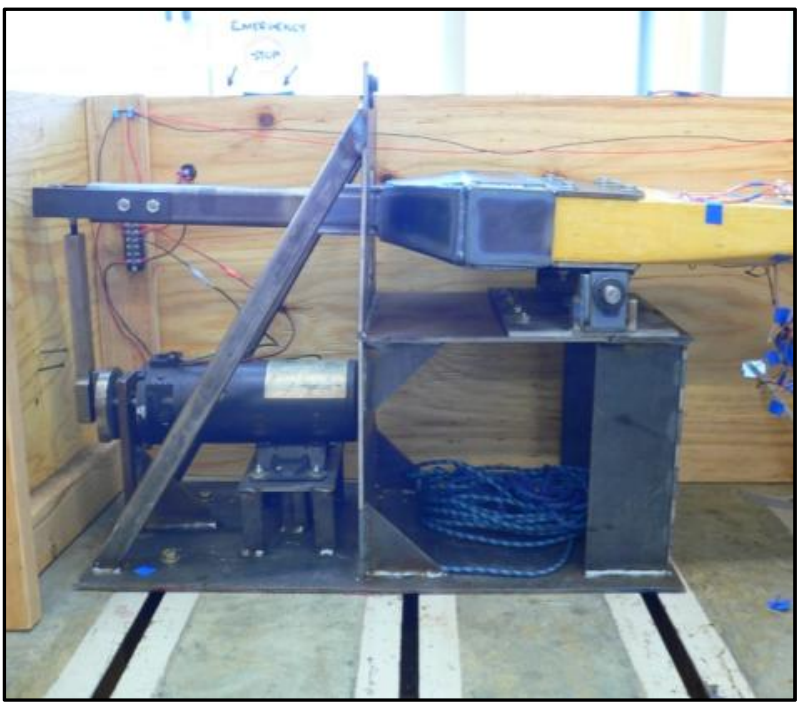

(b)

Figure 4-16. Support frame and blade vibration fixture as designed within SolidWorks (a) and the as-built steel fixture (b).

\subsubsection{Driving Crank and Motor}

The method of driving the blade clamping fixture and providing a vibration input to the system was performed by an electric motor and a variable offset crack disk and connecting rod. The design for these components was the most critical in the design process since all driving loads would originate at the motor and need to be effectively translated into a vertical displacement of the blade clamping fixture. To begin, calculations were performed to size the electric motor needed for this application. By assuming a uniform cross section for the blade and a material density close to the wooden laminate, a simple cantilevered beam was approximated by using the lumped mass method. Basic equations from dynamics were used to approximate the inertia of the blade if the tip of the blade was displaced 20 inches from horizontal in 0.1 seconds and the reaction loads were determined. Using these loads and the geometry of the fixture, the input torque needed by the driving motor was calculated assuming a maximum crank disk offset of 0.5 inches. Then, by assuming a driving frequency close to the first natural frequency of the blade the total power needed by the blade was calculated. It was determined that an input power of 0.2 horsepower was needed for this simple model. Knowing that the calculations were 
only simple approximations of the actual loading, an oversized motor rated at 1.5 horsepower was selected. This was a DC brushed motor and had current rating of 10 Amps.

The crank disk and connecting rod were then designed based on the loads from hand calculations and a factor of safety of 2.5 was used on all loads. The crank disk was designed to include a variable offset from zero to 0.5 inches which would allow the amplitude of the driving force to be user controlled. The connecting rod was designed to accommodate a bearing and pin on the crank disk connection side and a ball joint on the blade clamp driving arm side. A bearing support was also added between the motor and crank disk to support the bending loads and allow only the torsional loads from the system to pass through. Figure 4-17 shows the crank disk, connecting rod and motor from the rear. The connecting rod attached to the driving arm of the blade clamp via a ball joint steel rod seen from the rear in this figure.

\subsubsection{Wooden Blade Protection Barrier}

A simple plywood barrier was constructed to protect the blade during testing. It was designed to hinge in the middle to allow access to the blade in between tests and allow the operator to perform benchmarks testing. The barrier also provided a structure for attaching various instrument. A cycle counter was attached to the plywood and the emergency stop wire switch was also attached to the plywood. These instruments will be discussed in the following sections. Figure 4-18 shows the protective plywood barrier and the complete vibration test fixture may be seen as well. 


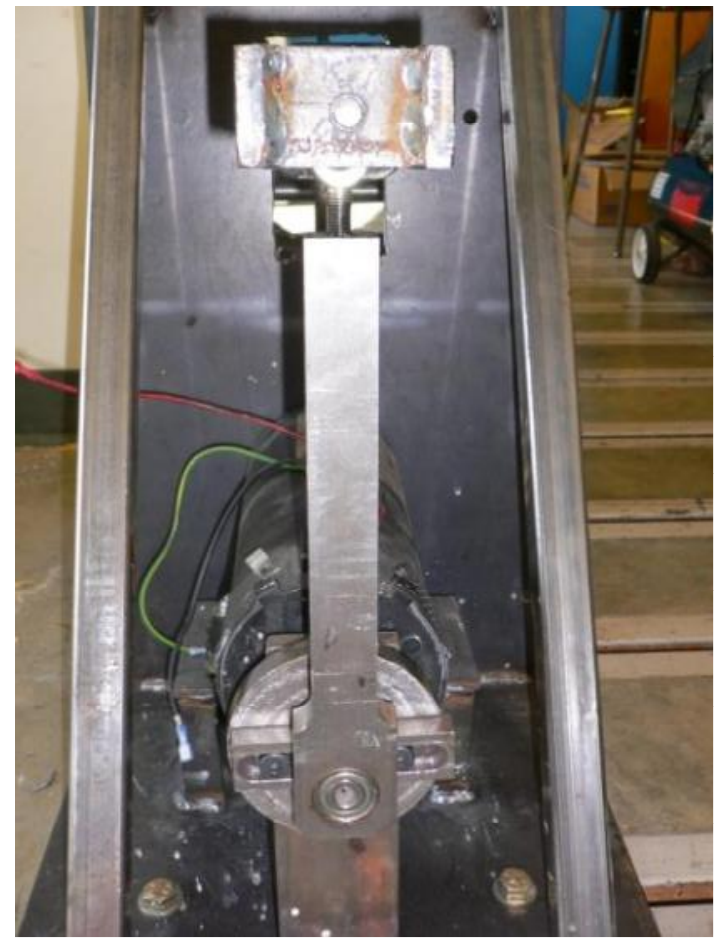

Figure 4-17. Crank disk and connecting rod used to drive the blade vibration fixture.

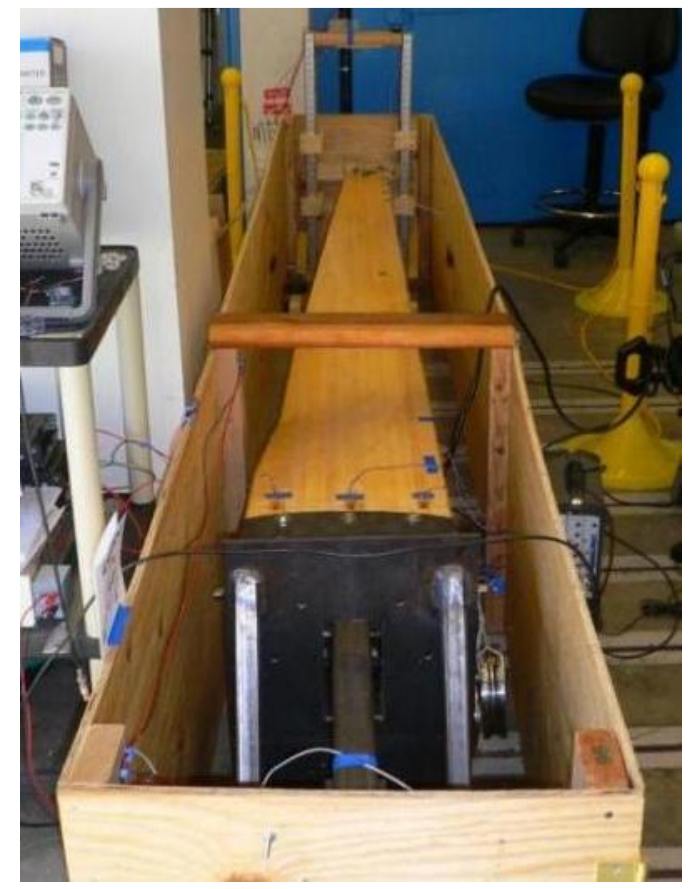

Figure 4-18 Full scale wooden blade fatigue life testing fixture showing wooden barrier constructed to protect the blade during testing. 


\subsubsection{Electronic Equipment and Circuits}

\subsubsection{Power Supplies}

The power for the motor was provided by a DC power supply and the motor speed was controlled by varying the input voltage manually. A Hewlett Packard 6543A DC power supply capable of supplying a 0-35 Volt and 0-6 Amp steady signal was obtained for this experiment. Control knobs on the front plate offered user control of these two values. Terminals on the front plate allowed positive and negative wires to be connected for the motor power supply.

One additional power supply unit was obtained from the Mechatronics lab at Cal Poly. This unit was capable of supplying a $5 \mathrm{~V}$ and $24 \mathrm{~V} \mathrm{DC}$ signal simultaneously. This unit was used to supply power to the electronic counter and Hall-Effect switch used to count the number of cycles completed during each test. These devices will be discussed in following sections.

\subsubsection{Motor Power Supply Circuit}

A simple on/off switch was incorporated into the motor supply circuit as well as an emergency stop fault switch. The fault switch was a simple terminal bus connected to the plywood barrier with wires hanging underneath the cantilevered blade. These wires were measured to a set length which allowed the blade to oscillate within a prescribed envelope of deflection without touching the wires. In the event of a blade or fixture failure, the blade would move past this pre-set distance and the terminals on the wires would be pulled out, thus breaking the circuit and cutting the supply voltage to the motor. Figure 4-19 shows the motor power supply circuit and with the various components labeled appropriately. All connections were made using 18 AWG wires and appropriately sized terminals for the current rating of the motor. Figure 4-20 shows the emergency stop fault switch bus terminals as attached to the plywood barrier. If a failure occurred, the wire terminals would pull out and voltage to the motor would be cut. 


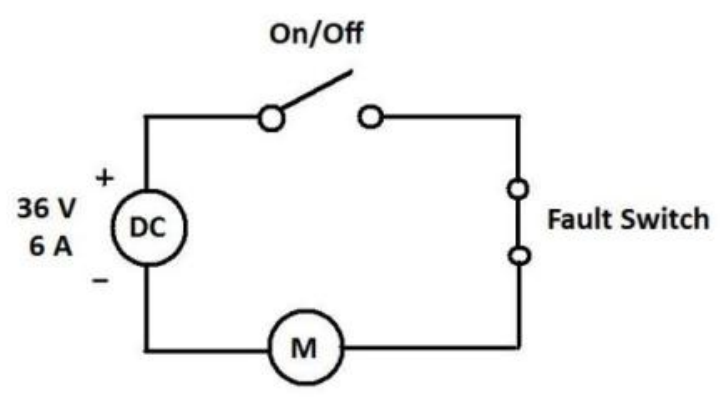

Figure 4-19. Motor power supply circuit showing on/off switch and fault switch.

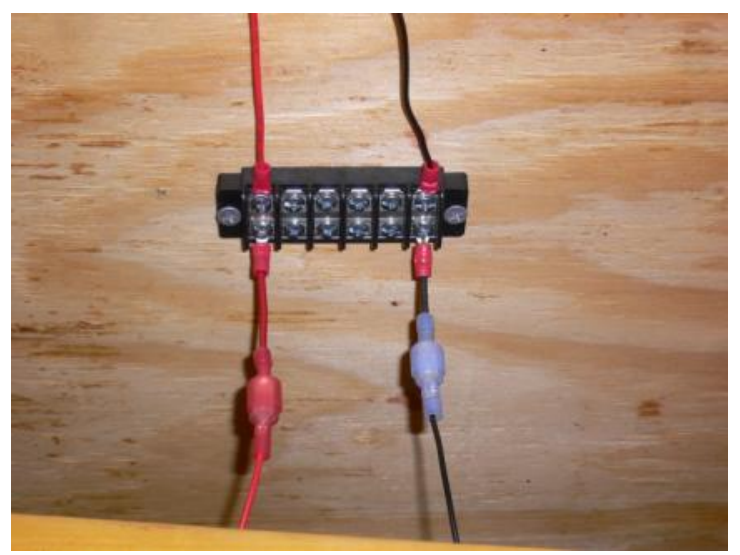

Figure 4-20. Picture of the blade fault switch attached to plywood barrier.

\subsubsection{Instrumentation}

The following sections will describe the various instruments used to collect data during each fatigue cycle test. Measurements of the blade tip deflection, blade root bending strains, input and output accelerations and blade oscillation cycle counts were collected.

\subsubsection{Blade Tip Deflection Measurement}

One of the primary validations for the numerical blade response code was the measurement of the actual blade tip deflection during fatigue loading tests. This measurement was completed using a simple visual measurement device created by the author. A wooden frame was built which supported two vertical identical metal rulers which were graduated in inches with an accuracy of $\pm 1 / 16$ of an inch. Four wooden clamps 
on the side of the frame could be slid up and down rails and the value at the current location could be visually inspected. Rubber bands were then stretched between two identical clamps and offered a means by which the current location of the blade could be measured directly. During operation, the clamps could be moved away from the blade and then slowly lowered until the rubber band began to vibrate due to small impacts from the blade. This allowed the amplitude of oscillation of the blade tip to be measured during every test to within a quarter of one inch accuracy. While this accuracy is somewhat coarse, the typical displacement of the blade tip was near 18 inches and this accuracy is offers less than $4 \%$ total error.

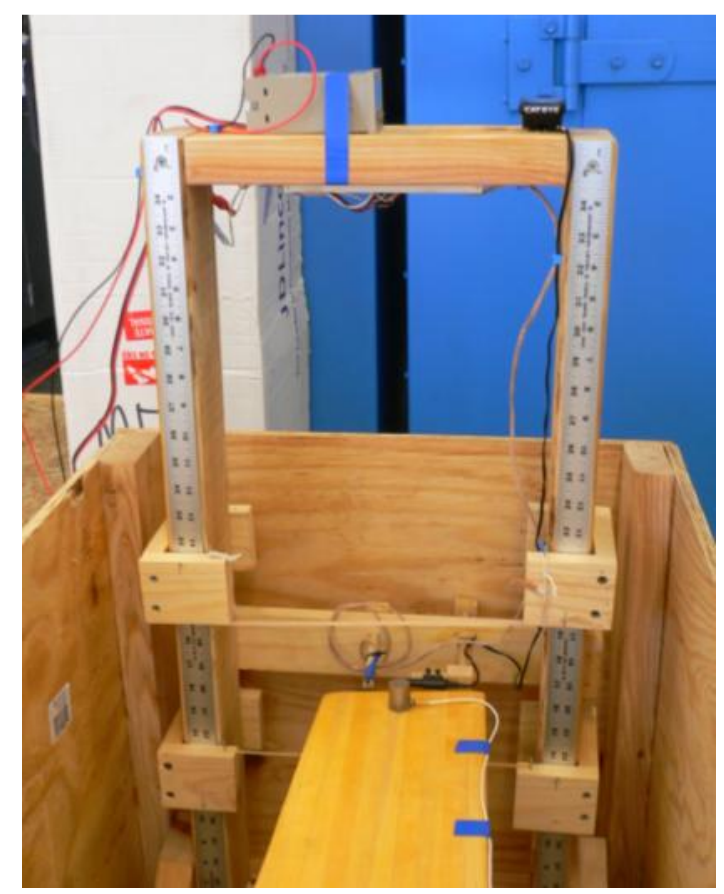

Figure 4-21. Blade tip deflection device showing vertical measurement graduations, wooden clamps and rubber band height indicators

\subsubsection{Cumulative Cycle Count Monitoring}

If any estimation of the cumulative fatigue damage accrued during testing was to be performed, an accurate measurement of the total cycle counts needed to be measured. Several methods were discussed and ultimately two cycle counting devices were chosen. Dual methods were employed since this measurement was crucial to the final prediction 
and certification of the fatigue life for the blades. A Hall-Effect sensor and electronic totalizer was selected and one method and simple bicycle computer was selected as the secondary method.

\subsection{Hall-Effect Cycle Counter}

The Hall-Effect sensor is a device based on the work of Edwin Hall who discovered that when a conductor with a known voltage applied, is moved perpendicularly through a magnetic field, a voltage difference is created on the top and bottom edges of the sensing conductor. This voltage difference can be measured and subsequently amplified to provide an accurate count of every time a magnetic field crosses the conductor's path. The HallEffect switch used for this experiment was actually an integrated circuit which combined a Hall-Effect sensor with a high gain integrated circuit to provide amplification of the sensing voltage for the digital counter box. An OMRON H7CR digital counter was obtained and the sensing inputs were connected to the Hall-Effect Switch. This counter needed a power supply of 24V DC and was supplied by the DC power supply mentioned in Section 4.4.2.1. The Hall-Effect switch also needed a power supply and the same 5V DC power supply was used. A small magnet was affixed to the blade tip and a fixture was built which held the Hall-Effect switch within 0.0625 inches of this magnet during testing. Every time the blade completed one cycle, the counter would sense four pulses from the Hall-Effect switch which were converted to four digital counts. This was because the sensor was able to detect the upper and lower magnetic flux lines emitted by the magnet. Accounting for this meant the total cycles counted by the box would need to be divided by four in order to determine an accurate cycle count. During actual blade testing at $6.5 \mathrm{~Hz}$, the digital counter box proved to be too slow to detect all four pulses and pulses were lost every cycle. As such, the bicycle computer proved to be a more reliable cycle counting device.

\subsection{Bicycle Computer Cycle Counter}

An inexpensive CatEye brand bicycle computer was purchased as a secondary cycle counter. This device works in a similar manner to the Hall-Effect sensor except the sensing component is a mechanical sensing device known as a Reed switch. A Reed switch is a small foil switch made from a ferro-magnetic material which is activated in the presence of 
a magnetic field. A second small magnet was attached to the blade tip and this sensor, mounted next to the Hall-Effect switch, counted every time the blade passed by. A bicycle computer normally calculates distance and speed by counting the number of times the sensor passes a magnet mounted on a bicycle wheel. To calculate cycles with this device, the wheel diameter of the theoretical bicycle was set to 1 meter so any distance calculated by the device would simply be equal to the number of cycles. The bicycle computer measured distance in kilometers so the values obtained were multiplied by 1000 to produce the real number of cycles. Similar to the Hall-Effect cycle counter, the bicycle computer counted two signals for every one complete cycle of the blade. As such, the total cycles were divided by two in order to account for this over estimation. Figure 4-22 shows the two sensors as they were mounted for testing and the small magnets attached to the tip of the blade.

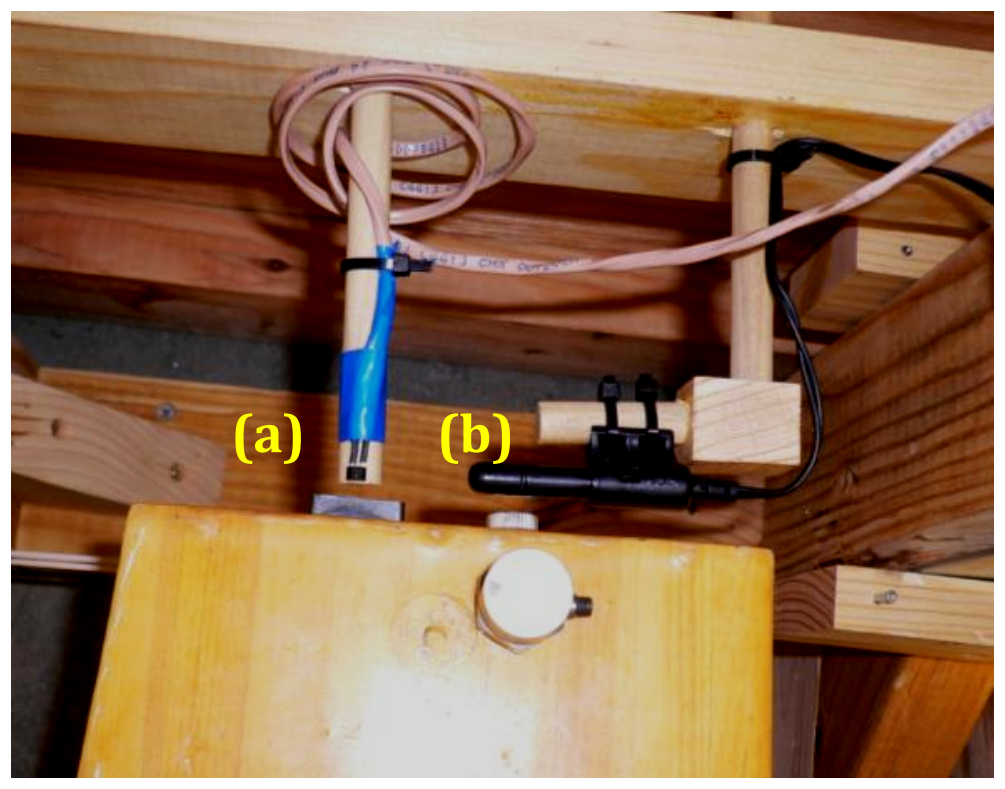

Figure 4-22. Orientation of the bicycle and Hall-Effect cycle count sensors. Magnets on the tip of the blade may be seen, a) is the Hall-Effect sensor and b) is bicycle computer sensor.

\subsubsection{Strain Gage Measurements}

In order to properly asses the fatigue damage during every cycle of the blade during testing, a measurement of the stress occurring at the critical section of the blade needed to be obtained. Attaching strain gages to the blade surfaces in question offers a Master's Thesis 
straightforward and accurate method for performing these measurements. If strain values are known then the stress values may be calculated using theory mentioned previously. A strain gage is a sensing device made from very thin foil laminates which is capable of detecting very small amounts of strain, which is defined as the ratio of the change in length of an object to the total length. When a voltage is applied to the gage, a change in resistance occurs if the gage is stretched along the main sensing axis and this resistance change can be accurately related to a change in length or strain. It was assumed that the critical section of the wooden blade was the root attachment point since the flapwise bending moment is greatest here. Hand calculations using a simplified cross-section confirmed this assumption and it was decided that several gages were to be placed at the root to monitor the strain during testing.

\subsubsection{Strain Gage Placements}

Two types of strain gages were utilized during the accelerated fatigue life testing of blade A and blade B: uniaxial strain gages and three axis strain rosettes. The uniaxial gages were used to measure the bending strains in the longitudinal axes and the strain rosettes were used to measure the principal bending strains at the root of the blade. Due to the data acquisition system limits, only four channels of strain data could be gathered at once so only the bending strains were measured during the accelerated fatigue life tests. Furthermore, the DAQ system could only measure half and full Wheatstone bridge configurations and the rosette gages required quarter bridge measurement capabilities. The uniaxial gages were bonded to the blade root in matching pairs which allowed these instruments to measure the maximum and minimum bending strains at the blade root. $\mathrm{A}$ half bridge strain gage circuit employs two active legs in the Wheatstone bridge and these legs allow only the change in length along the main gage sensing axis to be detected. Strain in the transverse direction on the gage is cancelled out by the paired characteristics of the circuit. These three strain gage pairs were used to document the alternating strain and thus stress occurring at the blade root. These values were then used to document the amount of fatigue damage which was induced by the accelerated fatigue life test. 
The rosette gages were used during the static deflections tests between the accelerated life tests to measure the principal strain values using a Vishay P3 strain indicator box. The rosettes used three gages which were mounted on the blade at $0^{\circ}, 45^{\circ}$ and $90^{\circ}$ angles with respect to the longitudinal axis. By measuring the strain in three directions at the same location, the principal strains for the material was calculated.

Blade A was the first blade to be tested and only the bending strains were recorded. Blade B was tested second and the bending strains as well as the principal strains were measured. The location of the strain gages on the root of the blade may be seen in Figure 4-23 as referenced from the leading edge of the blade. Table 4-5 shows the distances referenced in the figure and includes the gage type and Vishay product number.

Careful wiring of each gage was performed as directed by the Vishay strain gage installation guides. Each gage was attached to a connector block which allowed each gage to be individually connected to the Vishay P3 strain indicator box during the static loading tests. Figure 4-24 shows the gage locations, connecting wires and connector blocks used during the accelerated life testing of blade A. All connections were taped to the blade to avoid any vibration induced failures during the tests. 


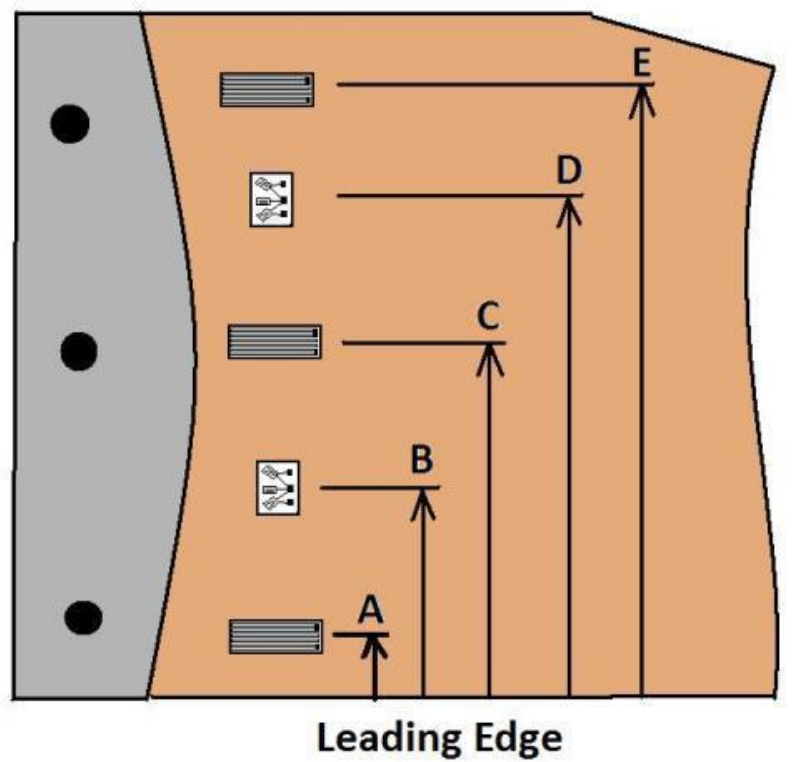

Figure 4-23. Illustration of strain gage locations for both uniaxial, (a, c, e) and rectangular rosettes (b, d) on the root of the blade.

Table 4-5. Strain gage designation and location as measured from the leading edge of the blade.

\begin{tabular}{|c|c|c|c|c|}
\hline \multirow{2}{*}{$\begin{array}{c}\text { Gage } \\
\text { Name }\end{array}$} & Blade A & Blade B & \multirow{2}{*}{ Gage Type } & \multirow{2}{*}{ Vishay Product \# } \\
\cline { 2 - 4 } & Location (in) & Location (in) & & \\
\hline A & 1.68 & 1.68 & Uniaxial & EA-10-250LZ-120 \\
\hline B & 3.05 & - & Rosette & CEA-10-060RZ-120 \\
\hline C & 6.13 & 6.13 & Uniaxial & EA-10-250LZ-120 \\
\hline D & 8.50 & - & Rosette & CEA-10-060RZ-120 \\
\hline E & 11.00 & 11.00 & Uniaxial & EA-10-250LZ-120 \\
\hline
\end{tabular}

Each strain gage pair was then wired together and connected to the data acquisition device using LEMO connectors. Each connector had six pins which were wired to the strain gages following the guides provided by manufacturer of the DAQ. A sample plot of the strain gage output during an accelerated fatigue life test may be seen in Figure 4-25. The strain gage values tended to fluctuate about a mean value with an error of plus or minus 4 micro strain. This error was small enough to not induce any noticeable trends in the data. Use of the DAQ will be discussed in the following sections. 


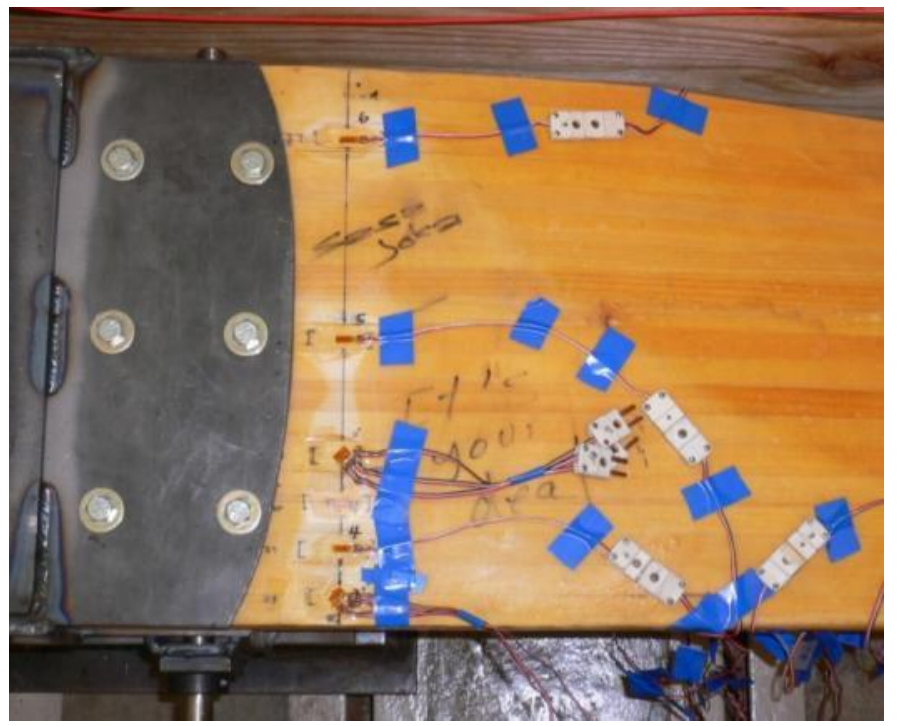

Figure 4-24. Strain gage connections as used during the accelerated fatigue life testing of blade A.

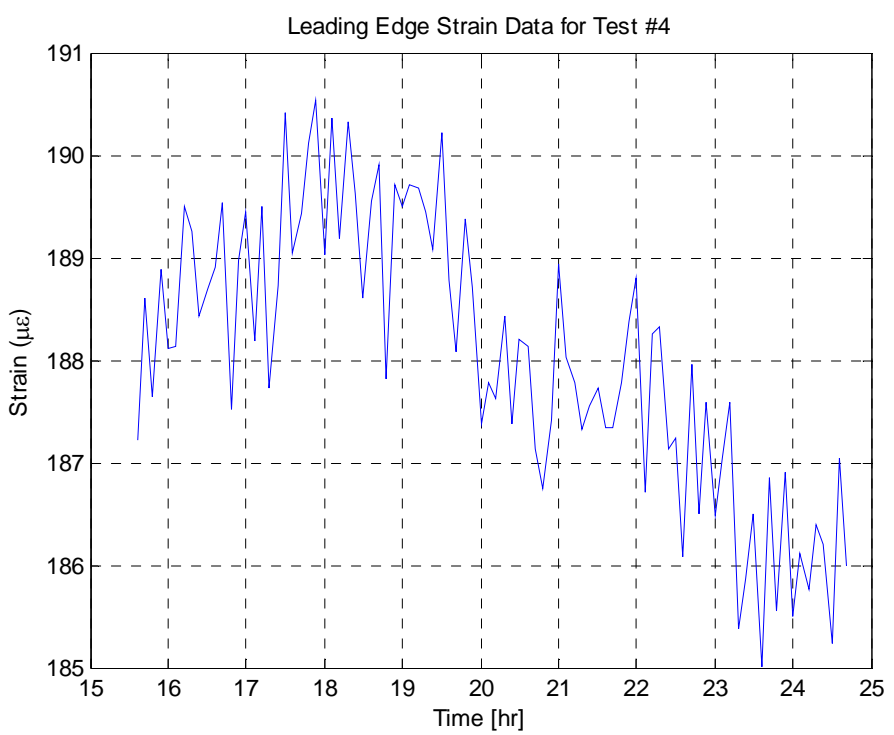

Figure 4-25. Example of strain gage output during accelerated fatigue life test number four for blade A. 


\subsubsection{Accelerometers}

A measure of the progressive damage occurring in the blade was performed by tracking the frequency response of the blade during the accelerated life testing. As shown by Bonfield et. al., the first natural frequency of a wind turbine blade decreases as progressive damage accrues and the wood fibers begin to break (Bonfield and Ansell 1993). By monitoring the input and output accelerations experienced by the blade, this frequency response can be studied. To this end, accelerometers were attached to the driving input crank arm on the steel fixture and the blade tip in order to monitor this frequency response. An accelerometer is a sensing device which measures an applied acceleration along a predetermined axis as referenced to freefall. There are many types of accelerometers and the devices used during this experiment utilized a small piezo-electric crystal which creates a voltage difference if applied accelerations impart a stress on the unit. These voltages are amplified by a charge amplifier and the voltage change is related to an acceleration based on the calibration of the device. Figure 4-26 shows the locations of the accelerometers used during testing.

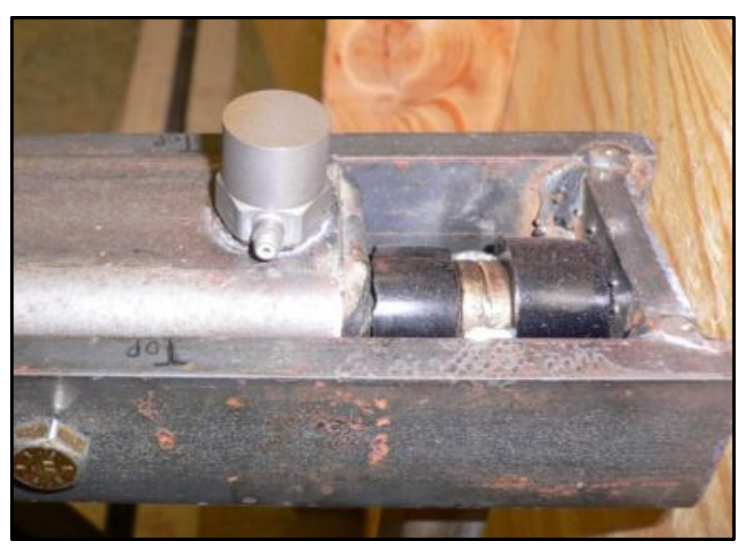

(a)

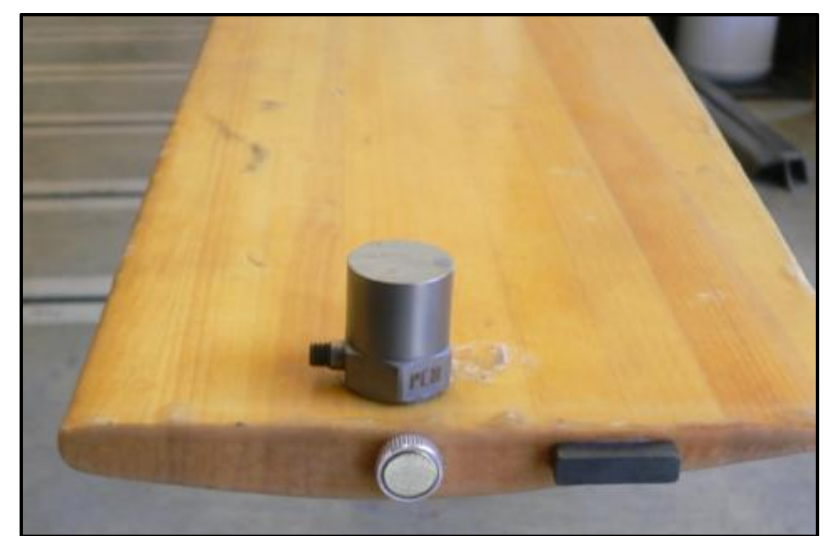

(b)

Figure 4-26. Accelerometers used during testing as mounted on the driving input arm, (a), and blade tip, (b).

If a measured acceleration is known at the input and output of a vibrating system, such as the blade input arm and blade tip, by calculating the ratio of these accelerations, the 
frequency response of the system can be monitored. Using a Fourier transform on the accelerometer signals and converting the time series data into the frequency domain, this ratio can be calculated and then shown to have peak amplitude at the vibrating frequency of the system. By monitoring the decreasing trend of this system's vibrating frequency, a measure of the fatigue damage was recorded. Furthermore, the amplitude of the input accelerometer signal as compared to the tip accelerometer gives a comparison of the tip deflection of the blade during the test. A known displacement was given by the crank disk of the fixture and this value was compared to the input accelerometer values. The blade tip acceleration was then compared to this value and by taking the second derivative of the tip accelerations, the displacement as a function of time was calculated. This measurement was used as a redundant measurement of the blade tip deflection to ensure this measurement was not lost during testing. Both accelerometers were connected to the DAQ system using a BNC connector from the charge amplifier.

\subsubsection{Testing Regime}

In accordance with the main objective of this project, an accelerated fatigue life test was performed on two wooden blades for the Gorman Controls' wind turbine. The testing process was divided into two parts: one being the actual accelerated life cycle testing and the second being static load tests which documented the progressive damage accumulated by each blade. A total of fifteen accelerated life tests were performed on blade B for a total of 144 hours and ten accelerated life tests were performed on blade A for a total of 98 hours. The goal of these tests was to reproduce the amount of wooden blade fatigue damage predicted using the numerical blade response model and the rainflow counted wind-stress cycles. The testing regimes for both blades are described in the following sections.

\subsubsection{Static Loading Tests}

At the beginning of both accelerated life tests and in between tests, static loading tests were performed. These tests involved locking the steel vibration fixture in a known position which allowed weights to be placed on the tip of the blade at a prescribed location. 
Weights were placed on the tip of the blade starting at zero pounds and increasing to fifty pounds in ten pound increments. The blade was allowed to reach an equilibrium position before measurements were recorded. Two measurement campaigns were completed during every static test: the bending strains at the root of each blade were recorded and the tip deflection from horizontal was recorded as well. Using basic equations from the theory of elasticity and the Euler beam bending equation, an effective stiffness was calculated based on the total deflection and strains measured during the test. These values were then recorded in between every duration test to track the progressive damage accruing in the blade. As the blade became more damaged the blade tip began to deflect more with the same amount of weight applied and the strain values increased in parallel as well. These tests served to demonstrate that the estimated fatigue damage totals calculated using Miner's Rule were correct by showing that the structure of the blade was damaged during the accelerated duration tests.

The static loading tests also served to validate the predicted tip deflection and stress levels which were calculated using the numerical blade response model. Figure 4-27 shows that the measured and predicted blade root flapwise bending stress as a function of prescribed tip deflection correlate well to one another. This correlation affirms that the numerical model correctly predicts the response of the blade to given wind conditions and gives justification for using the rainflow cycle counts and the derived S-N curve to determine the cumulative fatigue damage accrued during each duration test. 


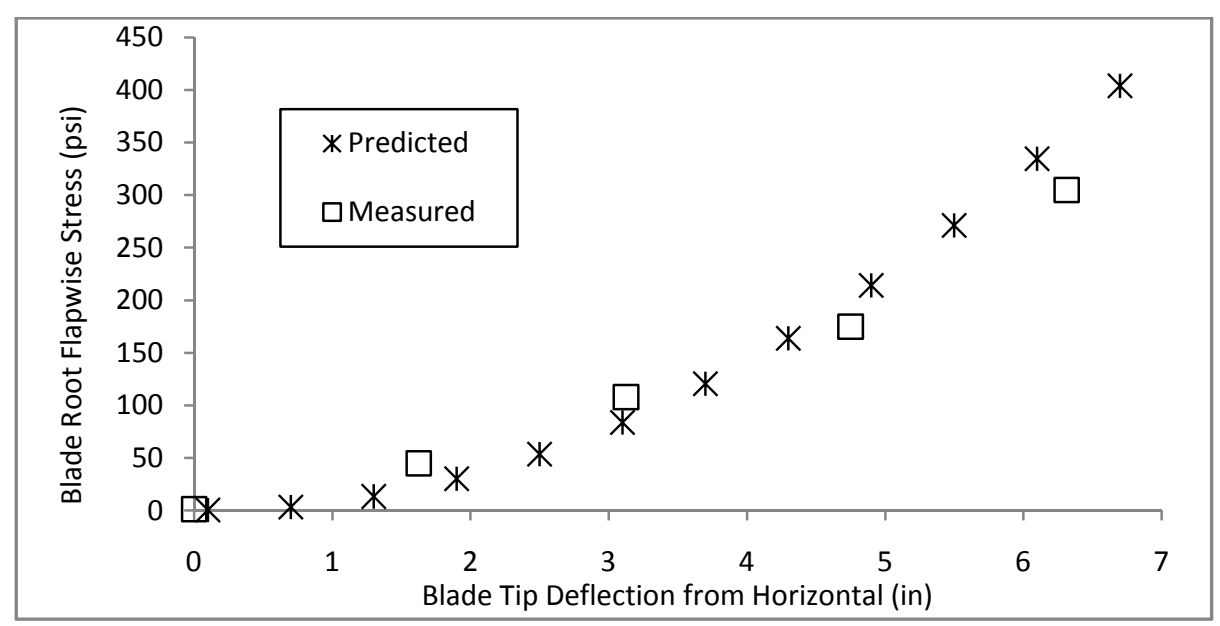

Figure 4-27. Comparison of the statically measured and predicted blade root bending stress as a function of tip displacement.

As shown in Figure 4-27, the numerical model under predicts the bending stress at low deflections and over predicts the bending stress at high deflections. This error may be attributed to differences between the as-built cross section of the blade and the profile used within the numerical model. While the profile used by the numerical model was based on actual dimensions measured from the as-built blades, the numerical profile was generalized out of necessity due to computer calculation constraints. Small imperfections within the wooden blade root may induce higher than predicted strains at small tip deflections and the generalizations used to simplify the calculation of the blade bending strains may under estimate the stiffness of the actual blade, thus giving higher than actual strain values at high tip deflections.

\subsubsection{Frequency Response Tests}

In parallel with the static loading tests and in between every accelerated duration test, a simple frequency response measurement was performed. Based on elementary vibration testing, a random vibration or "tap-test" was performed. This test involved tapping the tip of the blade and monitoring the damped response of the blade to this random excitation. An accurate measurement of the first natural frequency for the blade was obtained using a Fourier transform of the input and output accelerometer signals during this test. The nondimensional response amplitude based on these signals was calculated using a MATLAB Master's Thesis 
script and the natural frequency of the vibrating blade was calculated using this program. These tests served to document the decreasing trend in the first natural frequency for each blade which demonstrated that significant fatigue damage was accumulating during the accelerated fatigue life tests. Figure 4-28 shows an example plot obtained by using a MATLAB script written to calculate the damped oscillating frequency of the tapped blade. Results from all tests may be seen in section 4.5
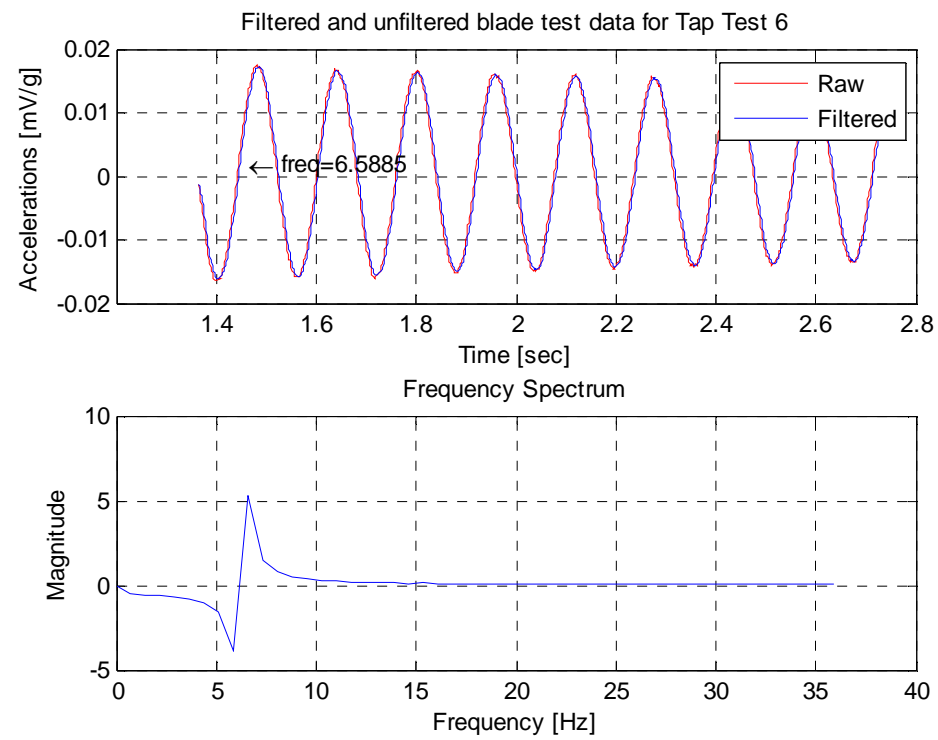

Figure 4-28. Example of tap-test response and MATLAB data analysis to find system natural frequency after duration test six for blade $B$.

The results from all of the sequential tap tests may be seen in the results section of this chapter. Figure 4-34 and Figure 4-35 show the decrease in natural frequency observed for blade $\mathrm{A}$ and blade $\mathrm{B}$ by performing the tap tests described above.

\subsubsection{Accelerated Fatigue Damage Tests}

In order to demonstrate that the wooden laminate blades for the Gorman Controls' wind turbine would survive fatigue loading during a 20 year service life, an accelerated fatigue life test was performed on two blades provided by the manufacturer. These blades were arbitrarily labeled A, B and C so all analysis and testing could be recorded with specific reference to each blade. Blade number B was randomly selected to be the first blade tested 
on the accelerated fatigue life fixture. The blade was subjected to a total of 3 million cycles of varying amplitude throughout a 144 hour duration test. This complete fatigue life cycle test was achieved with fifteen individual duration tests and a total of $24 \%$ cumulative damage was accrued by the blade during the test. Because the response of the fixture was unknown, the beginning few tests were run at lower amplitudes to prevent failure of the steel fixture from occurring. After several tests were completed and the fixture demonstrated that it could sustain repeated loading, the amplitude of the driving signal as well as the frequency was increased so the amount of fatigue damage would increase as well. Figure 4-28 shows the complete testing regime for blade number two and the cumulative damage total is highlighted at the bottom of the table.

Table 4-6. Duration testing regime for the accelerated fatigue life cycle test for blade number two.

\begin{tabular}{|c|c|c|c|c|c|c|c|}
\hline $\begin{array}{c}\text { Duration } \\
\text { Test }\end{array}$ & $\begin{array}{c}\text { Tip } \\
\text { Displacement } \\
\text { from Horiz. } \\
\text { (in) }\end{array}$ & $\begin{array}{c}\text { Per Test } \\
\text { Cycle } \\
\text { Count }\end{array}$ & $\begin{array}{c}\text { Stress } \\
\text { Level } \\
\text { (psi) }\end{array}$ & $\begin{array}{c}\text { S-N Curve } \\
\text { Predicted } \\
\text { Cycles }\end{array}$ & $\begin{array}{c}\text { Miner's } \\
\text { Rule } \\
\text { Cycle } \\
\text { Damage }\end{array}$ & $\begin{array}{c}\text { Progressive } \\
\text { Damage }\end{array}$ & $\begin{array}{c}\text { Cumulative } \\
\text { Cycles }\end{array}$ \\
\hline 1 & 2.50 & 64735 & 156 & 82909809 & 0.00078 & 0.00078079 & 64735 \\
\hline 2 & 1.25 & 313285 & 86 & 425314279 & 0.00074 & 0.00151738 & 378020 \\
\hline 3 & 2.50 & 473975 & 156 & 82909809 & 0.00572 & 0.00723414 & 851995 \\
\hline 4 & 2.63 & 166510 & 163 & 70403704 & 0.00237 & 0.00959921 & 1018505 \\
\hline 5 & 2.75 & 80115 & 170 & 59784019 & 0.00134 & 0.01093929 & 1098620 \\
\hline 6 & 2.75 & 353530 & 170 & 59784019 & 0.00591 & 0.01685274 & 1452150 \\
\hline 7 & 2.75 & 90890 & 170 & 59784019 & 0.00152 & 0.01837305 & 1543040 \\
\hline 8 & 4.00 & 60485 & 240 & 11654162 & 0.00519 & 0.02356304 & 1603525 \\
\hline 9 & 4.25 & 112560 & 254 & 8403501 & 0.01339 & 0.03695746 & 1716085 \\
\hline 10 & 4.25 & 297105 & 254 & 8403501 & 0.03535 & 0.07231236 & 2013190 \\
\hline 11 & 4.25 & 387960 & 254 & 8403501 & 0.04617 & 0.11847884 & 2401150 \\
\hline 12 & 4.50 & 203075 & 268 & 6059537 & 0.03351 & 0.15199213 & 2604225 \\
\hline 13 & 4.50 & 307165 & 268 & 6059537 & 0.05069 & 0.20268329 & 2911390 \\
\hline 14 & 4.50 & 9105 & 268 & 6059537 & 0.00150 & 0.20418588 & 2920495 \\
\hline 15 & 5.00 & 135885 & 296 & 3150633 & 0.04313 & 0.24731531 & 3056380 \\
\hline
\end{tabular}

Blade number one was tested second and a total of 1.9 million cycles of varying amplitude were applied by the steel fixture for a total of 98 hours. Ten separate duration tests were completed to achieve this cycle count and a total of $34 \%$ cumulative damage was accrued 
by the blade. Having already finished one complete accelerated fatigue life test, the testing of blade number one was accelerated more than that of blade number. This was done because a shorter complete test time period was desired. A much higher stress level was maintained throughout each duration test for blade number one as compared to similar tests for blade number two. The complete duration testing regime for blade number one may be seen in Table 4-7. The cumulative amount of fatigue damage is highlighted at the bottom of the table.

Table 4-7. Duration testing regime for the accelerated fatigue life cycle test for blade number one.

\begin{tabular}{|c|c|c|c|c|c|c|c|}
\hline $\begin{array}{c}\text { Test } \\
\text { Number }\end{array}$ & $\begin{array}{c}\text { Tip } \\
\text { Displacement } \\
\text { from Horiz. } \\
\text { (in) }\end{array}$ & $\begin{array}{c}\text { Each Test } \\
\text { Cycle } \\
\text { Counts }\end{array}$ & $\begin{array}{c}\text { Stress } \\
\text { Level } \\
\text { (psi) }\end{array}$ & $\begin{array}{c}\text { S-N Curve } \\
\text { Predicted } \\
\text { Cycles }\end{array}$ & $\begin{array}{c}\text { Miner's } \\
\text { Rule } \\
\text { Cum. } \\
\text { Damage }\end{array}$ & $\begin{array}{c}\text { Progressive } \\
\text { Damage }\end{array}$ & $\begin{array}{c}\text { Cumulative } \\
\text { Cycles }\end{array}$ \\
\hline 1 & 10.13 & 126305 & 560.9 & 4942533 & 0.0256 & 0.0256 & 126305 \\
\hline 2 & 10.13 & 226020 & 560.9 & 4942533 & 0.0457 & 0.0713 & 352325 \\
\hline 3 & 10.13 & 276230 & 560.9 & 4942533 & 0.0559 & 0.1272 & 628555 \\
\hline 4 & 10.50 & 205640 & 581.1 & 3944497 & 0.0521 & 0.1793 & 834195 \\
\hline 5 & 10.06 & 121755 & 557.6 & 5131873 & 0.0237 & 0.2030 & 955950 \\
\hline 6 & 9.88 & 73460 & 547.5 & 5744534 & 0.0128 & 0.2158 & 1029410 \\
\hline 7 & 9.75 & 235680 & 540.8 & 6193091 & 0.0381 & 0.2539 & 1265090 \\
\hline 8 & 10.00 & 263555 & 554.2 & 5328466 & 0.0495 & 0.3033 & 1528645 \\
\hline 9 & 9.50 & 266840 & 527.3 & 7198016 & 0.0371 & 0.3404 & 1795485 \\
\hline 10 & 9.75 & 107725 & 540.8 & 6193091 & 0.0174 & 0.3578 & 1903210 \\
\hline
\end{tabular}

\subsubsection{Data Acquisition Program and Methods}

As described in the above sections, measurements for the blade root bending strains and the input and output accelerations were desired measurements. Unlike the blade tip deflections or the cycle count totals, these measurements required a complicated PC based data acquisition system in order to capture the real-time data signals. An LDS Dactron Focus II data acquisition device was obtained from Dr. Charles Birdsong from the vibrations laboratory. The Focus II was a sophisticated DAQ system which allowed up to four channels of strain gage input and eight channels of analog voltage input to be monitored simultaneously. Sample rates above $5000 \mathrm{~Hz}$ were possible with the device but a sampling rate of $500 \mathrm{~Hz}$ was deemed acceptable for this experiment since the loading 
frequencies determined by the vibrating fixture was at least one order of magnitude lower than this. Either full or half-bridge strain gage configurations could be used by the device so three half-bridge strain gage channels from the blade root were collected during each test. The analog voltage signals sent by the two accelerometers were also collected during each duration test. A software interface to the Focus II called RT Pro Signal Analysis was provided by a PC laptop connection. This program allowed the user to specify the data channels to be recorded, the sample rates for each channel, the frequency of individual samples and the storage methods for these signals. After careful review of the user guides, a data extraction program was created which collected the data from each sensor and stored the files on the PC laptop.

\subsubsection{Data Storage Channels}

A total of five signals were recorded during every accelerated fatigue life test; the input accelerations, the tip accelerations, and three half-bridge strain gage signals. It was decided that a complete time history of the accelerated duration tests would be too data intensive and require more computer memory than was available. Instead, the five data channels were sampled every six minutes for a three second time period at the prescribed sample rate of $500 \mathrm{~Hz}$. This allowed an accurate measurement of the signals without storing massive amounts of data. A total of 1500 data points would be recorded during each three second sample and this data was then stored with a time stamp corresponding to every data point. Each channel of data was effectively sampled ten times every hour during each accelerated fatigue test and all five channels would be assigned an individual name based on the channel number and the time when the sample was taken. Data was collected by the PC laptop and each three second data sample was stored as a tab delimited text file for easy post processing. Table 4-8 shows the channel mapping used by the data acquisition system during each accelerated fatigue life test. 
Table 4-8. Data acquisition system channel mapping.

\begin{tabular}{|l|c|}
\hline \multicolumn{1}{|c|}{ Sensor } & $\begin{array}{c}\text { DAQ } \\
\text { Channel }\end{array}$ \\
\hline Input Accelerometer & 1 \\
\hline Blade Tip Accelerometer & 2 \\
\hline Strain Gage Half-Bridge A & 9 \\
\hline Strain Gage Half-Bridge C & 10 \\
\hline Strain Gage Half-Bridge E & 11 \\
\hline
\end{tabular}

The strain gages channels helped document the alternating stress level during every accelerated fatigue life test which was necessary in order to correlate these cycles to a particular amount of fatigue damage. As mentioned previously, the strain channels were used to verify the amplitude of the alternating stress so an accurate prediction of the total fatigue damage cause by the alternating cycles could be made. The accelerometers were used to monitor the amount of induced tip deflection and provide a measure of the frequency of oscillation of the blade. Each channel of data was extensively post processed using a MATLAB script written by the author which is described in the following section.

\subsubsection{Data Post Processing}

Once all fifteen duration tests for blade $\mathrm{B}$ and all ten duration tests for blade A were completed, the numerous text files stored by the DAQ on the PC laptop needed to be analyzed. This process was completed using a MATLAB script written by the author. The program used each channel's numerous text files as input and performed individual analyses as prescribed in the following section.

\subsubsection{MATLAB DAQ Script}

There were two main tasks for the MATLAB script written to analyze the text files generated by the fatigue cycle tests. The first task was to gather each three second sample text file into a collective time history for each channel of data. The second task was to perform individual analyses based on the sensor type and desired output. Several versions 
of the script were created for analyzing the complete set of testing data and for analyzing only the tap-test data which was gathered between consecutive duration tests. The program script read each data file based on the test number, channel and blade being tested and then created a concatenated array which stored the entire library of test data. Each channel was run through a Fourier transform and filter which calculated the frequency of the alternating signal and removed noise in the data samples. A Hilbert transform was also used to calculate the mean value of each three second data file so each sample file could be related to a mean and alternating value.

Figure 4-29 shows a sample strain gage data trace during the accelerated fatigue life tests. The alternating value of the strain is seen to have equal positive and negative amplitudes and is observed to alternate about a mean value of zero. A similar loading pattern was observed for all strain gage channels during every test which demonstrates that all three strain gage channels had a zero mean value and alternating values which corresponded to a stress ratio of $\mathrm{R}=-1$. This was critical in the calculation of the cumulative fatigue damage for the blades which was based on the S-N curve generated for fully reversed loading.
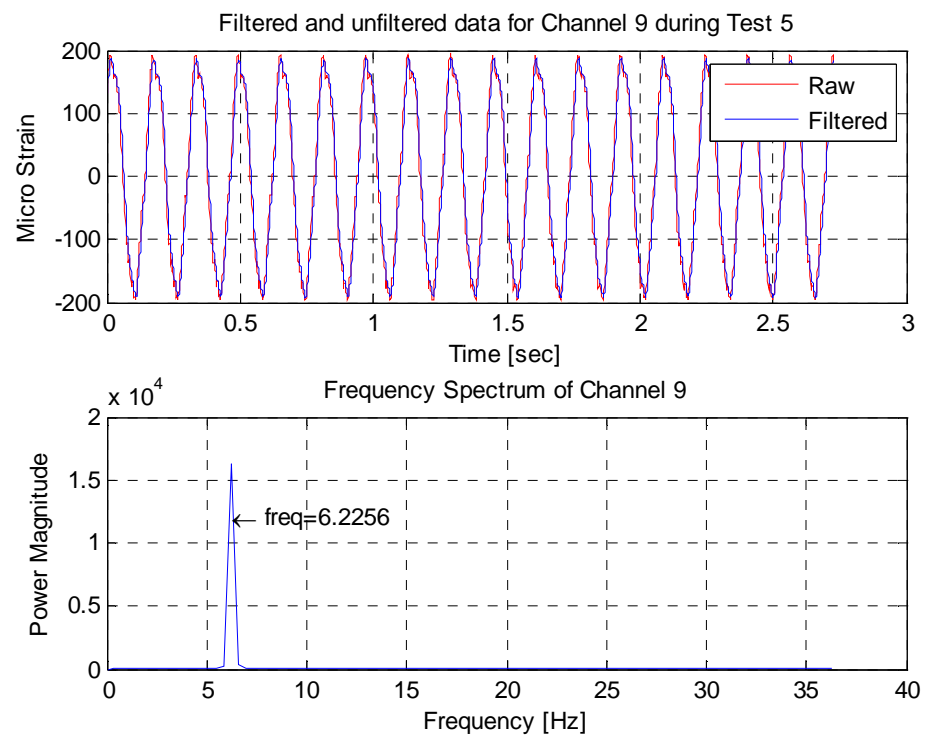

Figure 4-29. Validation of the assumption that the accelerated fatigue life tests subjected each blade to an alternating stress level equal to $\mathrm{R}=-1$. The strain level is seen to alternate from positive and negative 200 micro strain. 
As discussed previously, the amplitude of the tip deflection increased throughout the test because the driving frequency was increased. To correct for this signal offset, the strain gage channels 9,10 , and 11 were then normalized by the amplitude of the current tip deflection for each test. This was performed to show that the stress level was not increasing drastically during every test, rather only a small increase in stress was occurring. Furthermore, the amplitude of the accelerometers signal was corrected for this offset as well it was shown that the induced response of the blade remained about the same throughout the entire duration tests. A complete discussion of the results may be seen in section 4.5. The MATLAB script may be seen in Appendix A and sample plots created by the script may also be seen here.

\subsection{FULL SCALE FATIGUE LIFE TESTING RESULTS}

After completing the full life cycle test for each blade, an analysis of the recorded data was completed in order to quantify whether or not significant damage had accumulated within the blade itself. This analysis was performed using all of the data acquired through the strain gages, the between run benchmarks tests and the before and after static load tests. It will be shown in the following sections that a significant amount of progressive damage did occur within the blades during the complete testing process. The effective stiffness of each blade decreased as damage accumulated and the first fundamental frequency decreased as well. The decrease in these two properties indicates that the internal fibers of the blade were damaged due to the repeated loading regime imposed on the blade. The following sections will provide the details for each part of the analysis used to reach the aforementioned results.

\subsubsection{Individual DAQ Channel Results}

As mentioned in Section 4.4.5, each channel of data was concatenated into a complete array of data for the entire length of testing. This allowed the trends in strain gage data and accelerometer data to be analyzed. Figure 4-30 shows the entire data capture for the strain gage pair located at the leading edge, or position $\mathrm{A}$ on the blade root for blade $\mathrm{A}$. The figure shows each test number as a vertical red line with the test number preceded with the letter 
T. The strain gage signals were normalized based upon the tip deflection during each test to eliminate the bias due to an increase in testing driving amplitude. As shown in the figure, an increasing trend in stain gage alternating amplitude exists as the length of time the blade was tested increases. This shows that due to fatigue damage accumulating in the outer fibers of the blade, the strain levels increased. While this increase was not large, it offers an observable measure of the damage induced by the accelerated fatigue life tests and serves to verify the fatigue damage totals calculated. Plots for all three strain gage channels for both blade A and B may be seen in Appendix B.

Figure 4-31 shows the strain gage signal offset normalized by tip deflection observed between the individual tests performed during the accelerated life test of blade A. This offset demonstrates the increase in strain gage signal level observed to exist throughout the accelerated test. While the offset for strain gage pair A only increases by ten micro strain when not normalized, it nonetheless demonstrates that the strain levels increased due to fatigue damage accumulation. Similar results were observed for all three strain gage channels and the plots for each of these channels may be seen in Appendix B.

It is noted that several strain gage channels lost their signal during the accelerated testing process for blade B. The cause of this error was identified to be wiring malfunctions within the LEMO connectors but inspection of the connectors did not reveal a noticeable defect. Effort to correct the malfunction was made and as a result, the strain gage data capture during the second accelerated fatigue life test was much more reliable. 


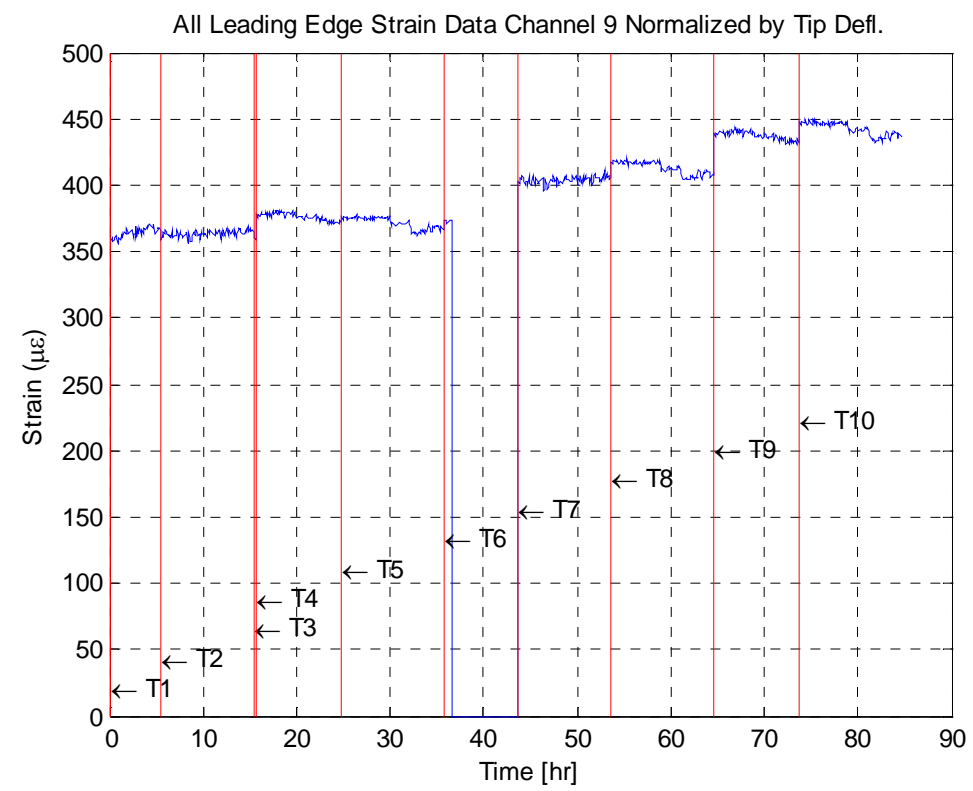

Figure 4-30. Plot of all strain gage letter A data for the entire testing life of blade A normalized by tip deflection distance. Note the loss of data after test six.

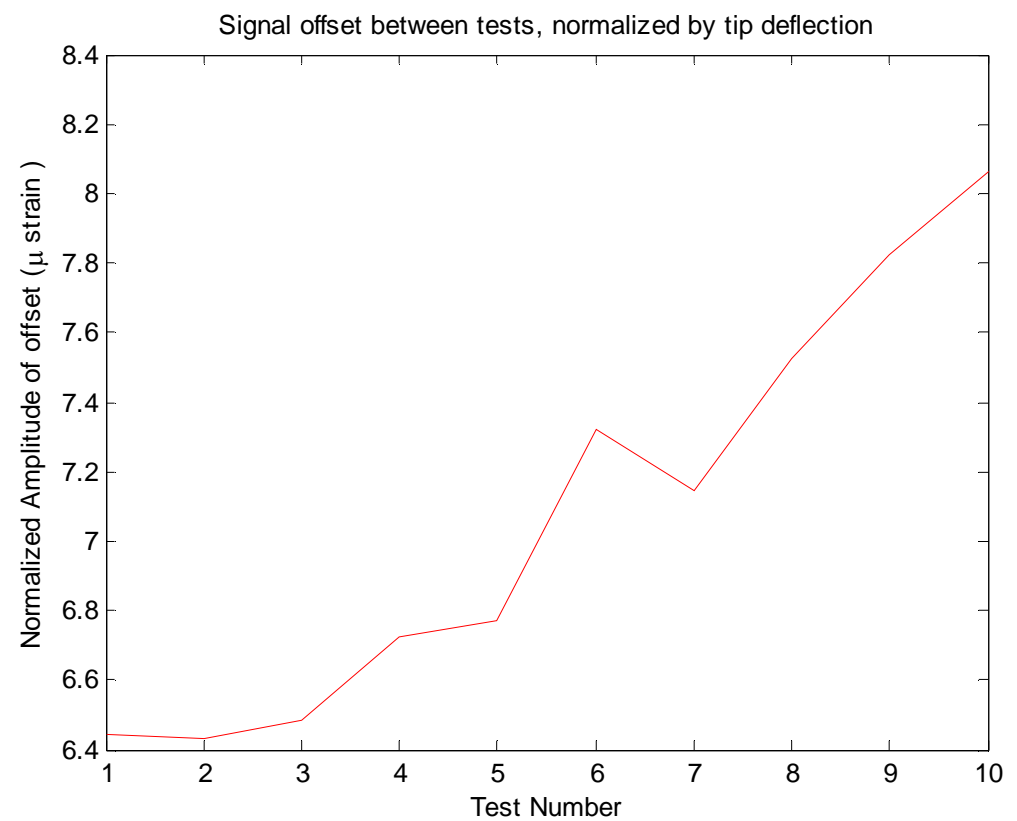

Figure 4-31. Strain gage signal offset observed between individual tests for strain gage pair A during the accelerated testing of blade A. 


\subsubsection{Cumulative Fatigue Damage Totals}

During every sequential accelerated fatigue life test the critical bending strains and cycle totals were documented. In accordance with the Palmgren-Miner Rule, if the alternating stress level is known and the cycle total is known, the amount of progressive damage can be assessed using the material's S-N curve. Having previously derived the S-N curve for the laminated pine blade in Chapter 3 and having previously defined the material properties of the wooden laminate, the critical strain was converted into a critical alternating stress using the theory of elasticity and the assumption that the wooden blade behaved as a transversely isotropic material. This work then enabled the amount of cumulative damage to be calculated for every consecutive accelerated fatigue life test. Figure 4-32 shows the amount of cumulative damage which was created within the blade during every consecutive accelerated fatigue life test. Blade B was the first blade to be tested and as such, the testing regime was less rigorous because the testing fixture had yet proven strong enough. The figure shows that a slow ramp up of fatigue damage was observed for blade B and a total of $24 \%$ cumulative damage occurred at the end of the test. Blade A was tested second and since the test fixture had demonstrated it could withstand the cyclic loading a much more aggressive testing regime was used. Figure 4-32 shows that a greater amount of fatigue damage was induced within blade A with fewer tests. The total fatigue damage for blade A was 34\% as shown in the figure. 


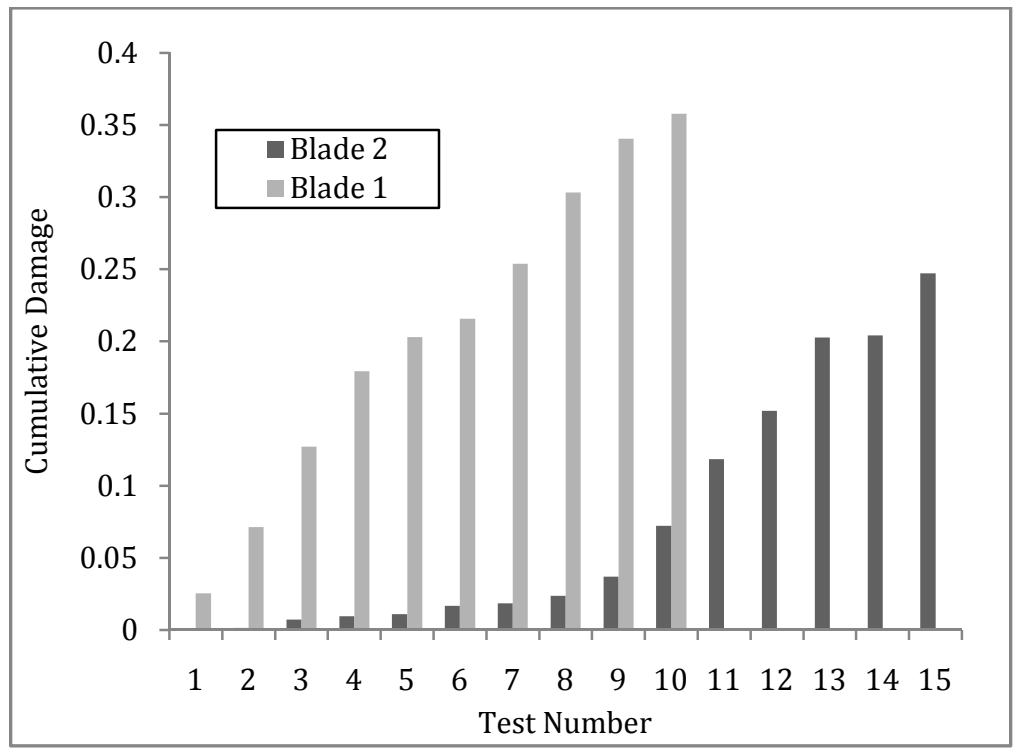

Figure 4-32. Illustration of total amount of fatigue damage accumulation during each sequential test for blade A and blade B.

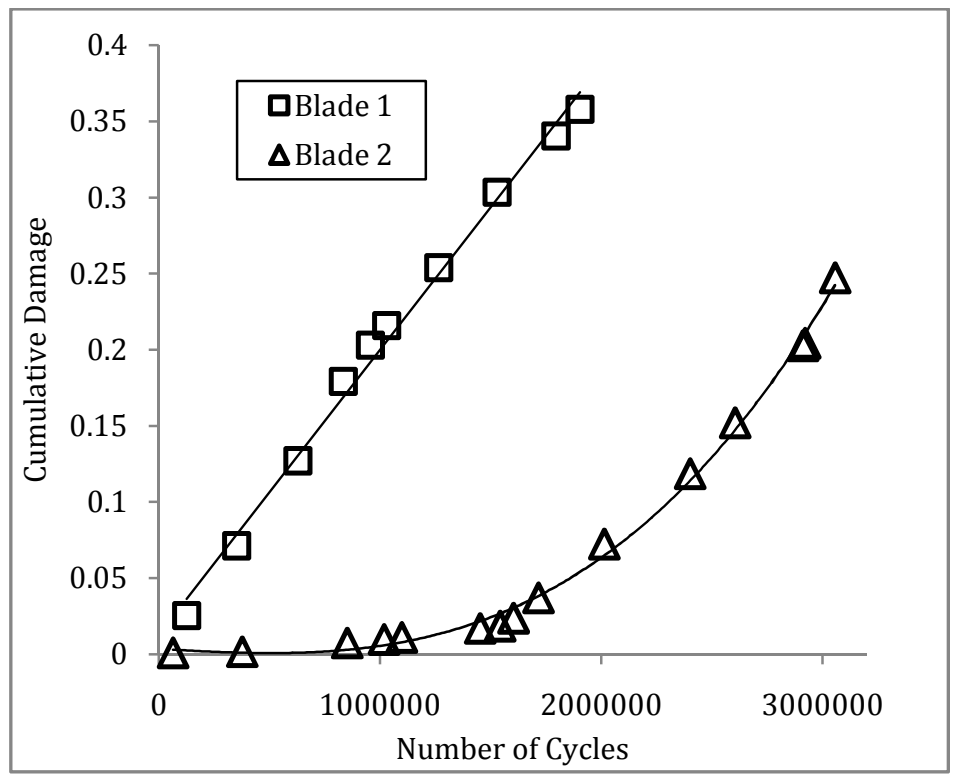

Figure 4-33. Cumulative damage as a function of total number of testing cycles for blade $\mathrm{A}$ and blade B.

Figure 4-33 demonstrates the rate at which fatigue damage was accumulated within blades one and two. Due to the more aggressive and higher stress amplitude during blade A's tests, the rate at which fatigue damage occurred was greater than blade B. This allowed a 
greater amount of fatigue damage to be induced with fewer cycles as shown in the figure. The regression curve fit lines are shown to illustrate that blade $\mathrm{A}$ was tested in a more rigorous and linear fashion than blade B. Blade B accumulated fatigue damage much slower than blade $\mathrm{A}$ and achieved a lower total amount of fatigue damage accumulation as well. While no suggestion of rate dependent fatigue cycle effects are noted, this may pose an area for further research. These effects may include adiabatic heating of the wooden fibers within the blade which have been shown to decrease the strength of wood during lab tests (Bond and Ansell 1998).

\subsubsection{Blade Response Degradation}

In between every consecutive accelerated fatigue life test, a series of static tests were performed. The equivalent stiffness of the blade was measured by placing weights on the tip of the blade and measuring the strains at the root. The natural frequency of the blade was also measured using a tap test method between each test as described in section 4.4.4.2. Both tests are described in Section 4.4. These tests served to document the effects of increasing the amount of cumulative damage within the wooden fibers of each wind turbine blade. Figure 4-34 and Figure 4-35 shows the decreasing trends in both the equivalent stiffness and natural frequency for blade number one and blade number two respectively. The natural frequency is observed to have a two stage decrease whereby an initial decrease in natural frequency is followed by a somewhat lower rate of decrease and which then increases towards the end of the test. The equivalent stiffness of the blade also exhibits a similar two stage decreasing trend as the natural frequency. Bonfield and Ansell showed that wooden composites used to manufacture wind turbine blades experience reductions in material properties, specifically the primary modulus of elasticity value $E_{1}$, at varying rates (Bonfield and Ansell 1993). Furthermore, when quantifying the rate at which the dynamic modulus of wooden coupons was observed to decrease they showed that an initial sharp drop in dynamic modulus was followed by a period of lesser decrease. Both the equivalent section stiffness and natural frequency of the wooden wind turbine blades tested in the accelerated fatigue life test fixture demonstrated these decreasing trends. This demonstrates that each wooden wind turbine blade manufactured by Gorman Controls experienced a measurable amount of fatigue damage during the cycle tests. Both 
blades demonstrated similar behavior during the accelerated cycle tests and both did not fail after an equivalent 20 year fatigue loading spectrum. This serves to prove that the wooden blade designed by Gorman Controls Ltd. will experience a fatigue life greater than 20 years.

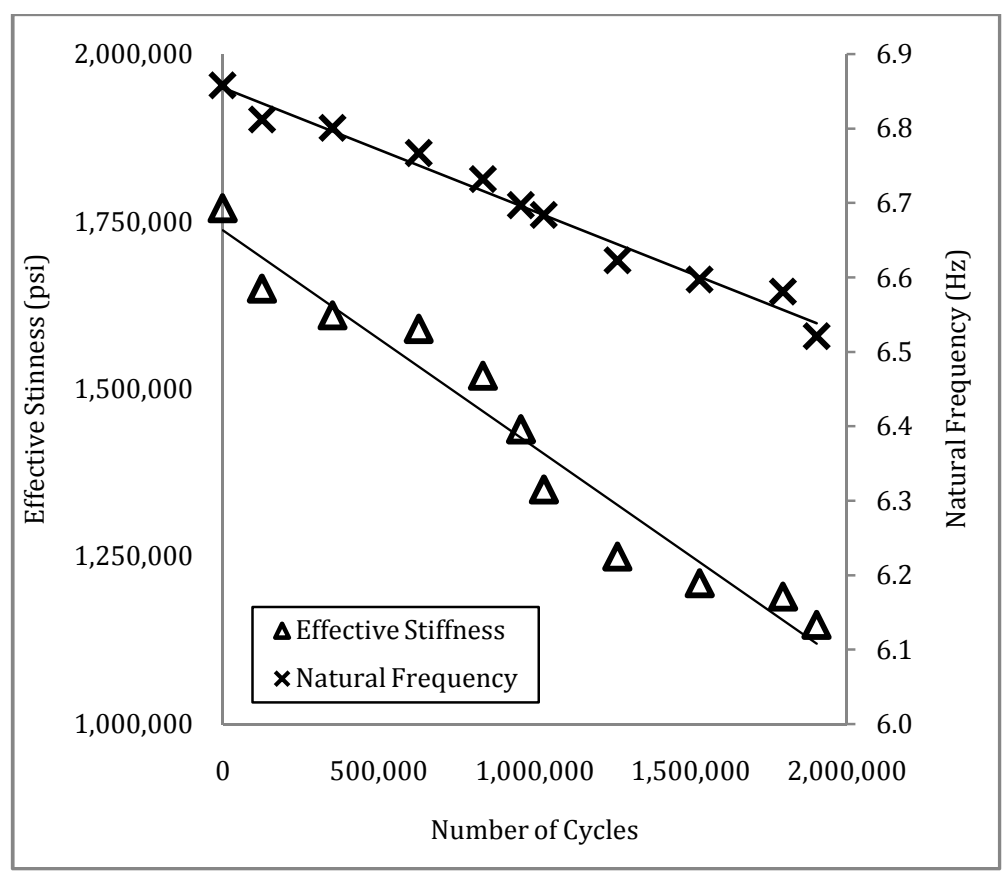

Figure 4-34. Observed decrease in effective stiffness and natural frequency during accelerated fatigue life test of Blade $A$. 


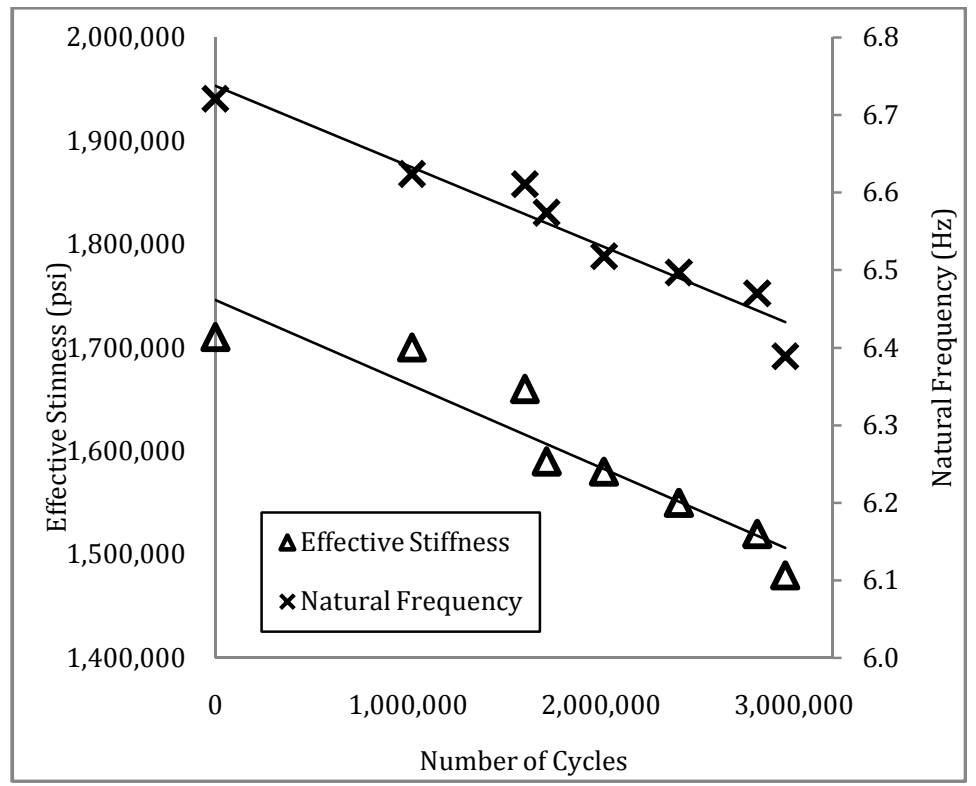

Figure 4-35. Decrease in effective stiffness and natural frequency observed during accelerated fatigue life test of Blade $B$.

Table 4-9 provides a summary of the beginning and ending parameters used to qualify the amount of fatigue damage which occurred in each wooden wind turbine blade during the accelerated fatigue life tests.

Table 4-9. Results from accelerated fatigue life tests performed on blade A and blade B.

\begin{tabular}{|c|c|c|c|c|c|c|}
\hline Blade & $\begin{array}{c}\text { Total } \\
\text { Cycles }\end{array}$ & $\begin{array}{c}\text { Cumulative } \\
\text { Damage }\end{array}$ & $\begin{array}{c}\text { Start } \\
\text { Equivalent } \\
\text { Stiffness (psi) }\end{array}$ & $\begin{array}{c}\text { Ending } \\
\text { Equivalent } \\
\text { Stiffness (psi) }\end{array}$ & $\begin{array}{c}\text { Starting } \\
\text { Natural } \\
\text { Frequency } \\
(\mathrm{Hz})\end{array}$ & $\begin{array}{c}\text { Final } \\
\text { Natural. } \\
\text { Frequenc } \\
\mathrm{y}(\mathrm{Hz})\end{array}$ \\
\hline 1 & $\begin{array}{c}1,903,21 \\
0\end{array}$ & 0.341 & $1.77 \mathrm{E}+06$ & $1.15 \mathrm{E}+06$ & 6.85 & 6.52 \\
\hline 2 & $\begin{array}{c}2,952,29 \\
0\end{array}$ & 0.247 & $1.71 \mathrm{E}+06$ & $1.48 \mathrm{E}+06$ & 6.72 & 6.39 \\
\hline
\end{tabular}

The amount of cumulative damage calculated to have been accumulated by each blade during the tests was based on the alternating stress values obtained from the strain gage pairs mounted on the root of each wooden blade. The material's S-N curve was validated using published data and experimentally measured values for ultimate strength. A 95th 
percentile lower bound was placed on the ultimate compressive strength of the material which served to make the fatigue damage estimate more conservative. The total number of cycles used to create this amount of fatigue damage was based on the rainflow cycle counted wind spectrum obtained from the Wind Energy Institute of Canada. It was shown in Chapter 3 that a total of 6.3 million stress cycles of varying amplitude occurred within a yearlong period. These cycles also shown to equal a total fatigue damage accumulation on $21 \%$ when multiplied by a desired 20 year total lifespan. Both blade A and blade B were tested to fatigue damage total greater than the predicted amount, therefore, the wooden wind turbine blades on the Gorman Controls' $10 \mathrm{~kW}$ wind turbine will have a fatigue life greater than 20 years. 


\section{Chapter 5. Conclusions and Recommendations}

\subsection{SUMMARIZED CONCLUSIONS}

To begin the process of estimating the fatigue life of a wooden wind turbine blade, the author successfully created a numerical model of the blades for the Gorman Controls, Inc.'s $10 \mathrm{~kW}$ wind turbine system. This model investigated the effects of gravity, centripetal accelerations and the effects of steady state aerodynamics on the blades due to set wind conditions. The maximum flapwise bending stresses at the root of the blade were predicted and this location was identified as the critical section with respect to fatigue loading. The blade response as a function of rotor azimuth angle was completed and shown to be effective at predicting when the blade would experience the largest flapwise bending moment during one rotor revolution. The rotating and non-rotating natural frequencies of the blade were predicted and were shown to be $7.5 \mathrm{~Hz}$ and $12.4 \mathrm{~Hz}$ respectively. This stiffening effect for the rotating blade serves decrease the chance that a blade's first natural frequency is excited during operation since the maximum rotor speed is set at $275 \mathrm{rpm}$ which is more than two and half times below this frequency. An estimation of the blade tip displacement out of the plane of rotation was provided by the model and used as a method of validating the numerical code when compared to the tested deflection of the real blade during static tests. Good agreement was found with respect to the calculated stresses from the program and the measured stresses derived from strain gage measurements at root of the blade. This served to further validate the model predictions and allowed the creation of a master curve relating wind velocities to stresses in the flapwise direction at the root of the blade. This curve was used to predict the number of fatigue causing cycles based rainflow counting.

A prediction of the maximum number of fatigue causing cycles during one year of use was successfully created based on a data file from the 2004 calendar year which was obtained from the Wind Energy Institute of Canada. Ten minute sample averages of wind velocity from a 30 meter tower were rainflow cycle counted to assess the total number of wind cycles occurring during the one year time period. It was shown that these cycle counts 
could be fitted to a statistical Weibull distribution with good accuracy and allowed further analysis of the total number of stress cycles which may be experienced by the blades on a wind turbine. It was shown by J. Eaaparachchi that a statistical estimation for the total number of fatigue causing stress cycles on the blade could be estimated using the original Weibull shape and scale factors determined from cycle counting the wind profile. Following this method, a matrix displaying the total number of fatigue damage causing cycles at all wind levels and alternating stress aptitudes was developed. The total number of fatigue causing cycles was determined to be 7,211,150 for a 20 year period of use. This number represents only the cycles which would induce a significant amount of fatigue damage and do not include the low amplitude cycles which may be induced due to the harmonic response of the blade. Using this matrix of total fatigue cycles and the master curve predicting blade root flapwise bending stress for all wind conditions, an estimation of the total progressive fatigue damage accumulated by a blade during 20 years of operation was determined to be just $21 \%$ of the total fatigue life of the blade. This estimation was then validated by performing a full scale accelerated fatigue life test on the blade.

In parallel with this work, a study of the manufacturing variability was completed using a 3D laser part scanner and the theoretical ideal blade profile provided by the manufacturer. A complete three dimensional model for all three blades sent to Cal Poly by the manufacturer were produced using the laser part scanner. Comparison of the ideal Clark Y profile used to design the blade with the as-built profiles showed good correlation with respect to chord length as the largest deviation was under 5\%. The cross sectional areas and section thickness values were shown to have much more error and some thickness values were shown to have larger than $30 \%$ difference in comparison to the designed profiles. This error can be attributed to the extremely thin trailing edge of the theoretical Clark Y profile near the tip of the blade and the inability of the manufacturer to achieve trailing edge thicknesses less than $1 / 16$ of an inch.

A study of the material properties for the wooden laminates was also completed and the results showed good agreement to published values for eastern white pine specimens. 
Validation of the material properties was a significant accomplishment since all stiffness and mechanical behavior of the blade predicted by the numerical code was directly linked to these values. Calculation of the maximum bending stresses assumed that the wooden laminate was well defined as a transversely isotropic material which allows the plane stress in the fiber direction to be expressed using only the modulus of elasticity and the measured strain in the fiber direction. Validation of this assumption was achieved by directly measuring the principal strains at the root of the blade and verifying that transverse shear at this location was very close to zero.

A full scale fatigue life experiment was successfully completed for two blades from the Gorman Controls' small wind turbine system. Detailed measurements during dynamic testing of the flapwise bending strains, tip displacement, total cycles completed, and tip accelerations were documented throughout the tests. Static load tests and harmonic response tests were also completed in between testing runs as a means of documenting the progressive damage accumulation. Each blade was driven at a specific amplitude corresponding to a higher than normal stress level and tip deflection near the first fundamental frequency of the blade. In this manner, an accelerated amount of fatigue damage was accrued in the blade and the rate at which this increased was monitored. Using the material S-N curve derived based on material property testing and research, the complete amount of fatigue damage was calculated to be $34 \%$ for blade A and $24 \%$ for blade $\mathrm{B}$ as compared to the total amount of fatigue damage each blade was capable of sustaining. These results certify each blade with a fatigue life greater than 20 years as was the main objective of this work.

\subsection{RECOMMENDATIONS}

The work presented here provided many results which were directly linked to several simplifying assumptions and linear theories which may lead to an under prediction of the total fatigue damage possible during a 20 year operating life for the Gorman Controls' wind turbine blades. Several recommendations are summarized below for future investigations. 
The coating used to seal the wooden blades was a common two part epoxy resin common in the marine and sailing industry. While the short term properties of this coating include Ultra Violet light protection and water repellency, the coating's response to impact from foreign objects is unknown. If the coating were to be removed by constant impact from hail, snow or other particles, the wood fibers would be exposed to the elements. It is well established by the Forest Products Laboratory of the U.S. that the material properties, specifically the ultimate strengths of the wood, are greatly reduced if the water content within the wood fibers increases larger than $15 \%$. Further investigation into the life of the coating should be investigated in order to certify the 20 year design life.

The loads which were predicted on the blades of the turbine were calculated using a linear theory to approximate the typical response of the blade to wind loading conditions. Further study using a more refined model, specifically one of the validated commercial codes such as BLADED, would help to remove uncertainty in these predictions. Furthermore, no account of yaw motion or furling induced loads were accounted for since only loads at steady state operating conditions were considered. Response to wind shear should also be accounted for in these models. Future study of these loading parameters may show a greater amount of fatigue damage is possible.

An investigation of the coupling between edgewise and flapwise bending moments was not accounted for in this study and may also give insight into higher predicted amounts of fatigue damage. This motion is predicted to be the source of significant loading on similar size wind turbines and the tapered transition from rectangular blade root into an airfoil cross section is characteristic of geometries observed by other wind turbine blades to have this coupling effect.

A final recommendation for future work and possibly the most important, is the assessment of a second in-field blade loading test regime. Lacking the actual blade loads during normal operation meant that no matter the level of certainty in the predicted numerical loads, complete validation of the fatigue life estimation was not possible. Since this validation was the primary objective for this study, future work should focus on 
determining the in-field loading parameters before investigating other secondary sources of loads.

\subsection{FINAL REMARKS}

The work completed as part of this thesis has shown that the fatigue life estimation for the wooden blades on a small wind turbine is complicated but nevertheless possible using modern computer models and the theories associated with wind turbine blades. Furthermore, a method for experimentally testing the predicted fatigue life of these blades has been shown to correlate well with linear theories and this work is offered as a new method for assessing the progressive damage of small wind turbine blades. The methods of extracting power from the wind will always represent a complicated science and the work presented here serves to increase this body of knowledge. 


\section{Chapter 6. Appendices}

\section{Appendix A. Derivation of Equations}

\section{Derivation of Aerodynamic Equations}

A simple linear model generally attributed to Betz (1926) is often used to described to total power capture from an ideal wind turbine. This theory is based on a linear momentum theory first developed for analyzing the performance of early ship propellers. This linear analysis assumes a stream tube control volume similar to Figure 3-8 where the flow enters on the left and exits on the right, never crossing the stream tube boundary in between. The wind turbine is modeled by an actuator disk which creates a pressure discontinuity between section 2 and 3. The following analysis is based on the work found in Wind Energy Explained by J. F. Manwell, J. G. McGowan and A. L. Rogers. The following assumptions are used in conjunction with the model:

1. No frictional drag.

2. Homogeneous, incompressible, steady state flow.

3. Uniform thrust over the rotor disk area.

4. Turbine has an infinite number of blades.

5. The flow wake does not rotate.

6. The static pressure far up and downstream are equal.

If the conservation of linear momentum is applied to the control volume the resulting thrust, $\mathrm{T}$, on the wind turbine can be found. This is found by the following equation:

$$
T=U_{1}(\rho A U)_{1}-(\rho A U)_{4}
$$

where $\rho$ is the density of air, $A$ is the cross sectional area and $U$ is the wind speed at each location indicated in Figure 3-8. Using the steady flow assumption necessarily means that the mass flow rates at locations one and four are equal, $(\rho A U)_{1}=(\rho A U)_{4}=\dot{m}$, where $\dot{m}$ is the mass flow rate. This leads to a simplified thrust equation given by: 


$$
T=\dot{m}\left(U_{1} \cdot-U_{4}\right)
$$

Because the thrust is positive and oriented parallel to the outward normal to the exit plane, the velocity at the exit plane must be less than the free stream velocity. Also, no work is done on either side of the wind turbine rotor so the Bernoulli function may be applied to the two control volumes. This is given by the following two equations:

$$
\begin{aligned}
& p_{1}+\frac{1}{2} \rho U_{1}{ }^{2}=p_{2}+\frac{1}{2} \rho U_{2}{ }^{2} \\
& p_{3}+\frac{1}{2} \rho U_{3}{ }^{2}=p_{4}+\frac{1}{2} \rho U_{4}{ }^{2}
\end{aligned}
$$

The thrust on the actuator disk may also be derived from the net forces acting at the disk surface assuming the velocity across the disk is the same and the free stream and downstream pressures are the same, namely:

$$
T=A_{2}\left(p_{2}-p_{3}\right)
$$

Using Equations (6.3), (6.4) and (6.5) and solving for ( $\left.\mathrm{p}_{2}-\mathrm{p}_{3}\right)$ the thrust on the actuator disk is then described by:

$$
T=\frac{1}{2} \rho A_{2}\left(U_{1}^{2}-U_{4}^{2}\right)
$$

If Equation (6.6) is equated to Equation (6.2) the wind velocity at the rotor plane is seen to be the average of the free stream and downstream velocities:

$$
U_{2}=\frac{U_{1}+U_{4}}{2}
$$

If an axial induction factor, $a$, is defined as the fractional decrease in wind velocity between the free stream, $U_{1}$, and the rotor plane, $U_{2}$ :

$$
a=\frac{U_{1}-U_{2}}{U_{1}}
$$


The the thrust on the actuator disk may be referred to using only the free stream wind speed, $\mathrm{U}_{1}$, by the equation:

$$
T=\frac{1}{2} \rho A U_{1}^{2}[4 a(1-a)]
$$

where $\rho$ is the density of air, A is the area of the actuator disk, $U$ is the free stream wind velocity. Knowing that the power output of the actuator disk is simply the thrust times the wind velocity at that surface, the power can be calculated using Equation (6.9) to be:

$$
P=\frac{1}{2} \rho A U^{3} 4 a(1-a)^{2}
$$

This rotor power can then be used to create a non-dimensional power coefficient, $\mathrm{C}_{\mathrm{p}}$, which is defined as the ratio of the rotor power to the total power available in the wind:

$$
C_{p}=\frac{P}{\frac{1}{2} \rho U^{3} A}=\frac{\text { Rotor Power }}{\text { Power in the Wind }}
$$

This may also be expressed using the axial induction factor as:

$$
C_{p}=4 a(1-a)^{2}
$$

To find the maximum value possible for the power coefficient, the derivative of equation (3.13)is taken with respect to $a$ and set equal to zero which yields $a=1 / 3$. Substituting this value for $a$ then gives the maximum rotor power coefficient as:

$$
C_{p, \max }=16 / 27=0.5926
$$

This derivation shows that the maximum power capture of an ideal wind turbine is limited to about $2 / 3$ of the total energy available in the wind and is known as the Betz limit. This linear model is only applicable for axial induction factors less than 0.5 because complicated turbulent flow occurs at values of $a$ greater the 0.5 which are not represented by this model. This ideal power capture is not possible in real life applications for three main reasons: rotation of the fluid flow occurs behind the actuator disk, a wind turbine has a finite number of blades and the drag on the airfoil is a non-zero quantity. 
The thrust on this linear ideal wind turbine can also be expressed by a similar nondimensional coefficient found by the following equation:

$$
C_{T}=\frac{T}{\frac{1}{2} \rho U^{2} A}=\frac{\text { Rotor } \text { Thrust }}{\text { Dynamic wind force }}
$$

When the axial induction factor is has a maximum value of $1 / 3$ at maximum power for the model, the thrust coefficient has a value of $8 / 9$.

The simple linear theory created by Betz can be improved upon by accounting for the rotating fluid flow created by the reaction of the fluid with the rotating blades. This analysis takes its roots from the generalized momentum theory first developed by Glauert to study the rotating propellers on early aircraft (Glauert 1935). Figure 6-1 shows the rotation of the fluid flow past the rotor plane which is induced when the rotor reacts with the incoming wind and the torque produced by the rotor is reacted in the opposite direction of rotation by the fluid.

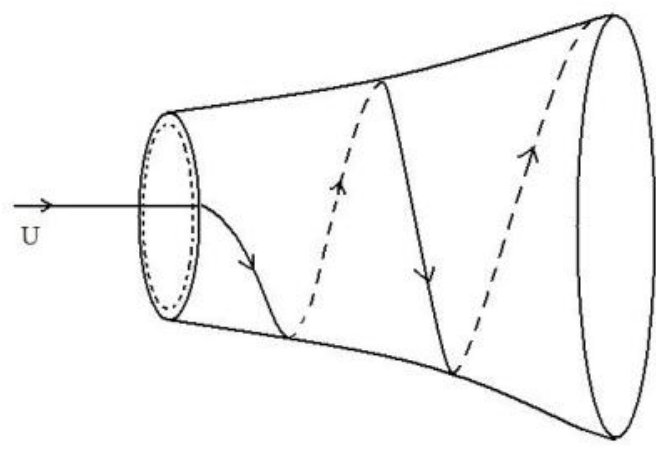

Figure 6-1. Example of stream tube including rotating wake past wind turbine rotor.

This new model the approximates the air flowing around the blades of a wind turbine with a stream tube of radius $r$ and thickness $\mathrm{dr}$ which can have an angular velocity, $\omega$. A differential thrust force acting on this annular ring of area within the stream tube is found to be: 


$$
d T=\rho U^{2} 4 a(1-a) \pi r d r
$$

Similarly, by applying conservation of angular momentum to the annular disk the differential torque imparted to the rotor is found by:

$$
d Q=4 a^{\prime}(1-a) \rho U \pi r^{3} \Omega d r
$$

where $\Omega$ is the angular velocity of the rotor and the angular induction factor a' is defined as the ratio of the flow angular velocity, $\omega$, and the rotor angular velocity, $\Omega$ :

$$
a^{\prime}=\frac{\omega}{2 \Omega}
$$

A final useful equation which is used to determine the maximum efficiency of a particular blade profile defines the ratio of the tangential velocity of the rotating blade tip, $\Omega \mathrm{r}$, with the free stream wind speed, $U$. This ratio is referred to as the tip speed ratio and is found by the equation:

$$
\lambda=\frac{\Omega r}{\mathrm{U}}
$$

where $\Omega$ is the angular velocity of the rotor.

\section{Blade Element Momentum Theory}

A theory known as blade element or strip theory allows further investigation of the forces on a wind turbine blade by accounting for the aerodynamics of the blades. This analysis accounts only for the aerodynamic lift and drag forces at various angles of attack determined by the aerodynamic properties of the airfoils. Figure 6-2 shows a typical blade as it would be sectioned into differential slices with the associated geometrical dimensions given for each section profile. 


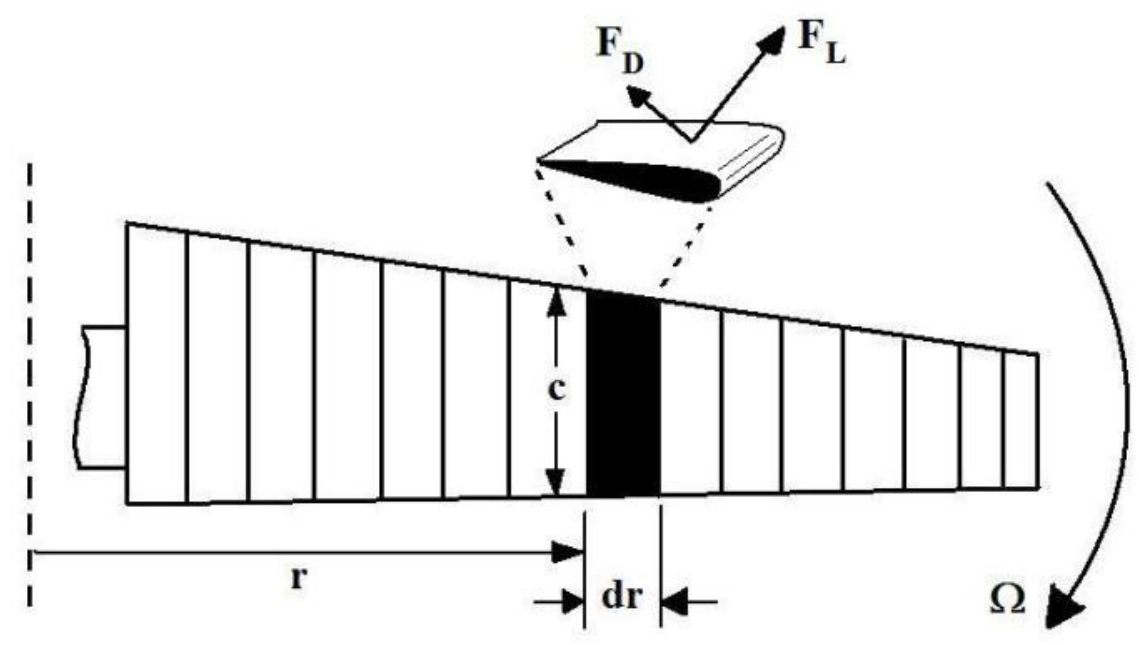

Figure 6-2. Diagram of wind turbine blade sectioned into individual blade elements. The rotor angular velocity is $\Omega, r$ is the radius of the section, $d r$ is the differential section thickness, and $c$ is the section chord length. The lift, $F_{L}$ and drag, $F_{D}$ forces are found for every airfoil section.

The blades are divided into $\mathrm{N}$ sections and the lift and drag forces associated with each section are calculated depending on the angle of attack of the airfoil with respect to the relative wind velocity. This analysis assumes that no interaction between the elements exists and that the forces on the blades only arise from aerodynamics. The differential lift and drag forces are which arise at each differential section are calculated based upon the aerodynamic loads generated by the profile:

$$
\begin{aligned}
d F_{L} & =C_{l} \frac{1}{2} \rho U_{r e l}{ }^{2} c d r \\
d F_{D} & =C_{d} \frac{1}{2} \rho U_{r e l}{ }^{2} c d r
\end{aligned}
$$

where $C_{l}$ and $C_{d}$ are the lift and drag coefficients for the airfoil, $U_{\text {rel }}$ is the relative wind velocity and $\mathrm{c}$ is the airfoil chord length of the differential section. Realizing that these lift and drag forces will both contribute to the differential thrust and torque on the rotor, components of these forces can be calculated based on the angle of relative wind. Referring to Figure 6-3, the differential forces in the edgewise and flapwise directions receive 
contributions from both the lift and drag on the airfoil based on the angle of the relative wind, $\Phi$.

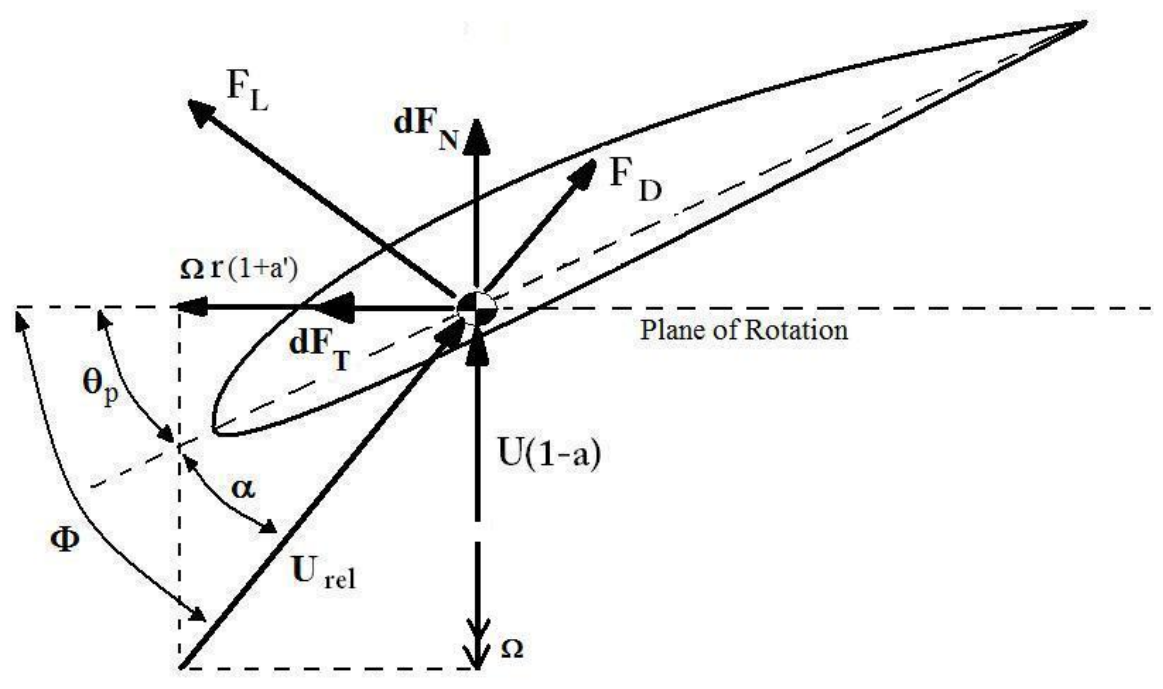

Figure 6-3. Induced aerodynamic forces due to a relative wind velocity, $U_{\text {rel. }} F_{L}$ is the airfoil lift force, $F_{D}$ is the airfoil drag force, $r$ is the radius of rotation measured from the hub center, $\Omega$ is the angular velocity of the rotor, $\alpha$ is the angle of attack, $\Phi$ is the angle of the relative wind, $\theta_{\mathrm{p}}$ is the section pitch angle, $a$ is the axial induction factor, $a^{\prime}$ is the angular induction factor, $\mathrm{dF}_{\mathrm{N}}$ is the differential normal or flapwise force and $\mathrm{dF}_{\mathrm{T}}$ is the differential tangential or edgewise force.

These differential forces may then be calculated based on the sine and cosine contributions as follows:

$$
\begin{aligned}
& d F_{N}=d F_{L} \cos \phi+d F_{D} \sin \phi \\
& d F_{T}=d F_{L} \sin \phi-d F_{D} \cos \phi
\end{aligned}
$$

The differential contributions in both the normal and tangential directions are then summed to find the complete thrust and torque experienced by the rotor due to the aerodynamics of the blades. The equations of blade element theory and momentum theory for the differential thrust and torque on the rotor may then be equated to one other to provide a complete description of the forces acting on the blades. If the lift and drag coefficients are known for the specific airfoil, then the forces on each differential section of blade may be calculated and ultimately the blade flapwise and edgewise bending moments 
may be totaled based on the thrust and torque predicted. It is useful to note that for a reasonable approximation of the loads on the blades of a wind turbine, the above mentioned values and formulas are the only required parameters. A more detailed model which accounts for additional degrees of freedom of the blades is described the following section.

\section{Hinge Spring Offset Model Equation Derivations}

While the theory of blade element momentum theory accounts for the main aerodynamic forces acting on the blades of a wind turbine, two other sources of loading are neglected, namely the effects of gravity and rotor inertia. The hinge-spring offset model first developed by Eggleston and Stoddard, accounts for all forces which may act upon the rotor of the wind turbine by modeling the blade as a beam with hinges and springs allowing motion in the edgewise, flapwise and torsional directions (Eggleston and Stoddard 1987). The flexible blade is then approximated by a rigid uniform cross section beam, attached via springs to the hub with a hinged offset. Figure 6-4 shows the standard nomenclature for the hinge-spring model including the coordinate system and azimuth angle, $\Psi$. The model assumes that the blade has a uniform cross section and is rigid and that the hinge is offset from the axis of rotation. The assumption that the blades spin at a constant speed is also made. 


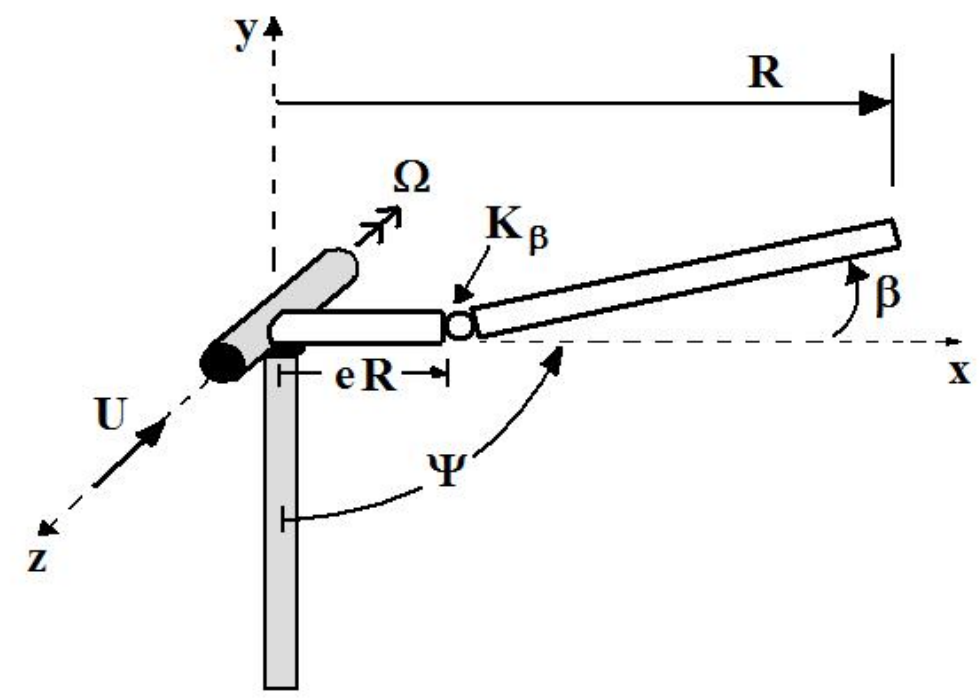

Figure 6-4. Illustration of the hinge-spring model of a wind turbine where $R$ is the blade radius, $U$ is the free stream wind speed, $e$ is the non-dimensional hinge offset, $K_{\beta}$ is the spring constant, $\beta$ is the flapping out of plane angle, $\Omega$ is the rotor angular velocity, $\Psi$ is the blade azimuth angle and the coordinate system $\mathrm{x}, \mathrm{y}, \mathrm{z}$ rotates with the blade.

To begin the analysis, the hinge offset and spring stiffness are calculated so the stiffness and rotating natural frequencies are the same as the real blade. The rotating, $\omega_{\mathrm{R}}$ and nonrotating, $\omega_{\mathrm{NR}}$ natural frequencies are first calculated for the real blade using a generalized beam method such as the Mykelstad method presented in section 3.3.3. Eggleston and Stoddard have shown that the non-dimensional hinge offset may be calculated using the following equation:

$$
e=2(Z-1) /[3+2(Z-1)]
$$

where $\mathrm{Z}$ is found by:

$$
Z=\left(\omega_{R}^{2}-\omega_{N R}^{2}\right) / \Omega^{2}
$$

Assuming that the blade has a uniform cross section, the area moment of inertia of the blade including the hinged offset is calculated as:

$$
I_{b}=m_{B}\left(R^{2} / 3\right)(1-e)^{3}
$$


where $m_{\mathrm{B}}$ is the mass of the blade. In general, the equation of motion for this system may be described as the sum of the blade restoring moments and forcing moments as a function of the blade flapping angle, $\beta$ :

$$
\ddot{\beta}+[\text { (restoring moments })] \beta=g(\text { forcing moments })
$$

where $\ddot{\beta}$ is the second derivative of the flapping angle with respect to time. If the system is first considered to be operating in a steady state condition the forcing moments reduce to zero and the equation of motion reduces to the following:

$$
\ddot{\beta}+\left[\Omega^{2}(1+\varepsilon)+\mathrm{G} \cos (\Psi)+\mathrm{K}_{\beta} / \mathrm{I}_{\mathrm{b}}\right] \beta=0
$$

where $\varepsilon$ is the offset term given by:

$$
\varepsilon=3 e /[2(1-e)]
$$

and $\mathrm{G}$ is the gravity induced moment given by:

$$
G=g m_{B} r / I_{b}
$$

where $m_{B}$ is the blade mass and $\mathrm{I}_{b}$ is the mass moment of inertia of the blade. As seen in the equation, there are three moments which act upon the blade and they derive their nature from the blade inertia, gravity and the spring constant introduced into the hinge respectively.

If the forcing moments induced by the wind are then included in the model, a function, $\mathrm{M}_{\beta}$ can be used to describe the forces previously derived using blade element momentum theory. The effects of yawing the turbine may also be accounted for using a yaw rate term, q. If the second partial derivative of Equation (6.25) is taken with respect to the azimuth angle, $\Psi$, and each term is divided by $\Omega^{2}$, a more general equation of motion is created. In summary, the complete equation of motion for the rotating wind turbine blade can be show to be: 


$$
\beta^{\prime \prime}+\left[1+\varepsilon+\frac{G}{\Omega^{2}} \cos (\Psi)+\frac{K_{\beta}}{\mathrm{I}_{\mathrm{b}} \Omega^{2}}\right] \beta=\frac{M_{\beta}}{\Omega^{2} I_{b}}-2 q \cos (\Psi)
$$

where $\beta^{\prime \prime}=\ddot{\beta} / \Omega^{2}$ is the azimuthal second derivative of the blade flapping angle $\beta, \varepsilon$ is the hinge offset term defined above, $\Psi$ is the blade azimuth angle measured counter-clockwise from bottom dead center, $K_{\beta}$ is the blade flapping spring constant, $q$ is the yaw rate, and $G$ is the gravity term.

Further manipulation of Equation (6.28) yields terms for the effects of gravity, wind shear, yaw rate and the hinge spring constants. While the complete solution of this equation was considered, a simplified model was used by the author whereby only gravity, hinge spring constants and flapping frequency calculations were performed. The yaw rate and wind shear terms were set to zero as per derivations provided by J. Manwell in Wind Energy Explained. The modified equation of motion were implemented in the numerical blade response model. 


\section{Appendix B. Additional Figures and Tables}

\section{Chapter 3. Figures and Tables}

Table 6-1. Rainflow cycle count totals of the WEICAN 2004 year long wind speed record.

\begin{tabular}{|c|c|c|c|c|c|c|c|c|c|c|}
\hline $\begin{array}{c}\text { Windspeed } \\
\text { Range (ft/s) }\end{array}$ & \multicolumn{9}{|c|}{ Windspeed Cycle Alternating Amplitude (ft/s) } \\
\hline & 2.15 & 3.46 & 4.77 & 6.09 & 7.40 & 8.71 & 10.02 & 11.33 & 12.64 & 13.95 \\
\hline 3.270 & 259 & 0 & 0 & 0 & 0 & 0 & 0 & 0 & 0 & 0 \\
\hline 7.509 & 701 & 18 & 0 & 0 & 0 & 0 & 0 & 0 & 0 & 0 \\
\hline 11.749 & 1472 & 53 & 15 & 0 & 0 & 0 & 0 & 0 & 0 & 0 \\
\hline 15.988 & 1924 & 67 & 21 & 8 & 0 & 0 & 0 & 0 & 0 & 0 \\
\hline 20.228 & 1786 & 80 & 21 & 10 & 11 & 0 & 0 & 0 & 0 & 0 \\
\hline 24.467 & 1534 & 72 & 21 & 16 & 9 & 16 & 0 & 0 & 0 & 0 \\
\hline 28.707 & 1311 & 76 & 16 & 7 & 3 & 9 & 5 & 0 & 0 & 0 \\
\hline 32.946 & 977 & 43 & 6 & 3 & 4 & 2 & 3 & 4 & 0 & 0 \\
\hline 37.186 & 755 & 25 & 6 & 1 & 0 & 1 & 0 & 2 & 2 & 0 \\
\hline 41.425 & 562 & 16 & 2 & 1 & 0 & 0 & 2 & 0 & 0 & 2 \\
\hline 45.665 & 422 & 8 & 0 & 0 & 0 & 0 & 0 & 0 & 0 & 1 \\
\hline 49.905 & 284 & 12 & 4 & 1 & 0 & 1 & 0 & 0 & 0 & 0 \\
\hline 54.144 & 180 & 10 & 2 & 0 & 0 & 0 & 0 & 0 & 0 & 0 \\
\hline 58.384 & 103 & 2 & 1 & 0 & 0 & 0 & 0 & 0 & 0 & 0 \\
\hline 62.623 & 74 & 6 & 0 & 0 & 0 & 0 & 0 & 0 & 0 & 0 \\
\hline 66.863 & 55 & 1 & 1 & 0 & 0 & 0 & 0 & 0 & 0 & 0 \\
\hline 71.102 & 34 & 2 & 0 & 0 & 0 & 0 & 0 & 0 & 0 & 0 \\
\hline 75.342 & 25 & 2 & 0 & 0 & 0 & 0 & 0 & 0 & 0 & 0 \\
\hline 79.581 & 19 & 0 & 0 & 0 & 0 & 0 & 0 & 0 & 0 & 0 \\
\hline 83.821 & 11 & 0 & 0 & 0 & 0 & 0 & 0 & 0 & 0 & 0 \\
\hline
\end{tabular}




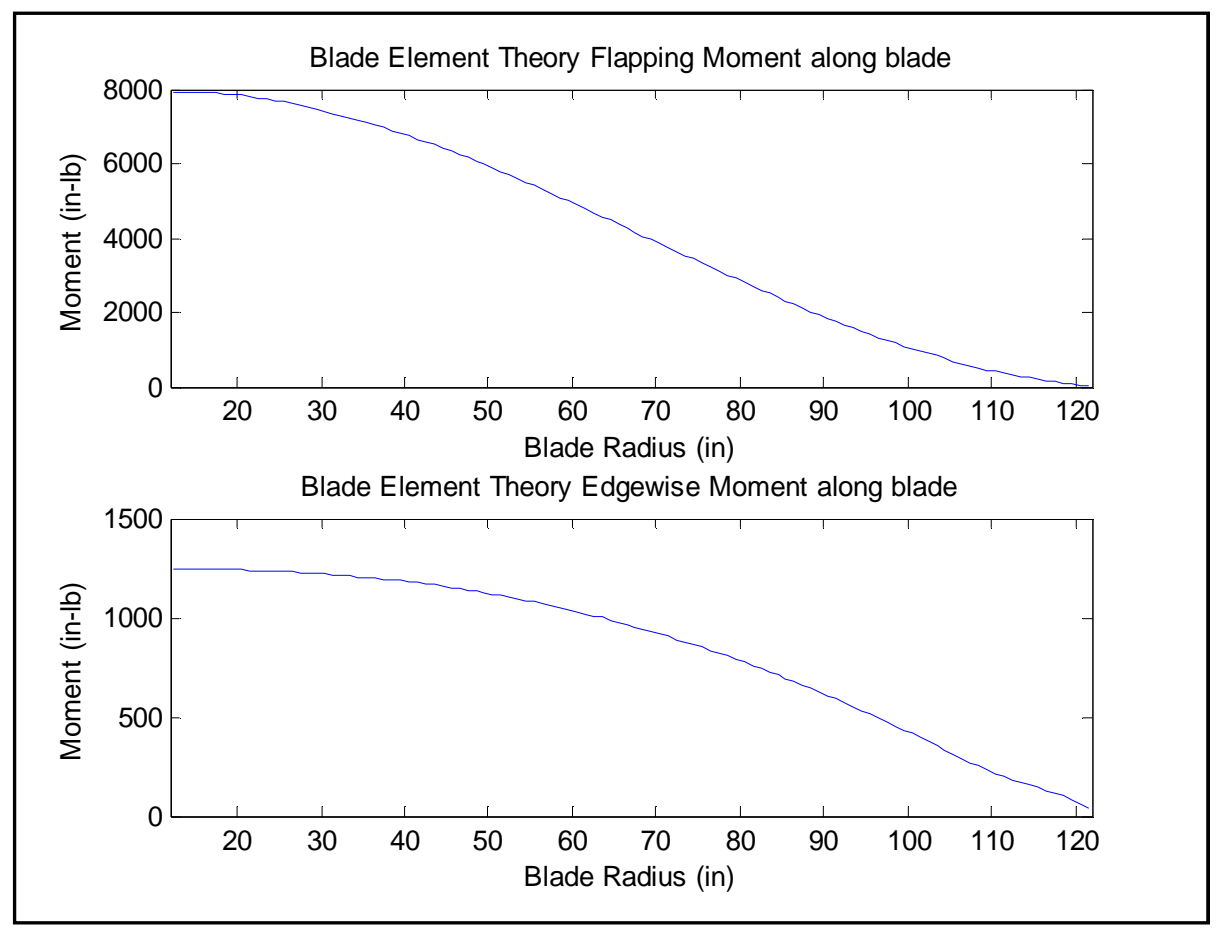

Figure 6-5. Numerical blade model predicted edgewise and flapwise bending moments.

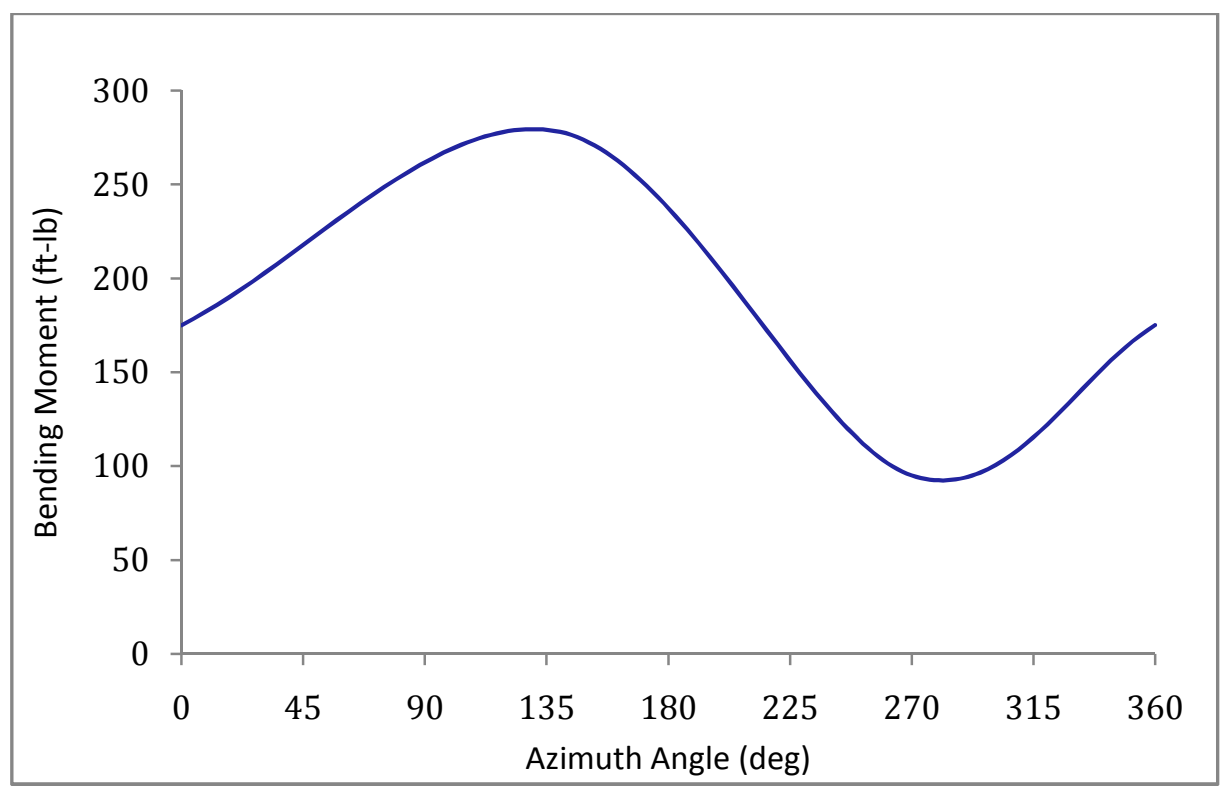

Figure 6-6. Example numerical blade response model predicted flapwise blade root bending moment as a function of blade azimuth angle at a rotor speed of $195 \mathrm{rpm}$. 


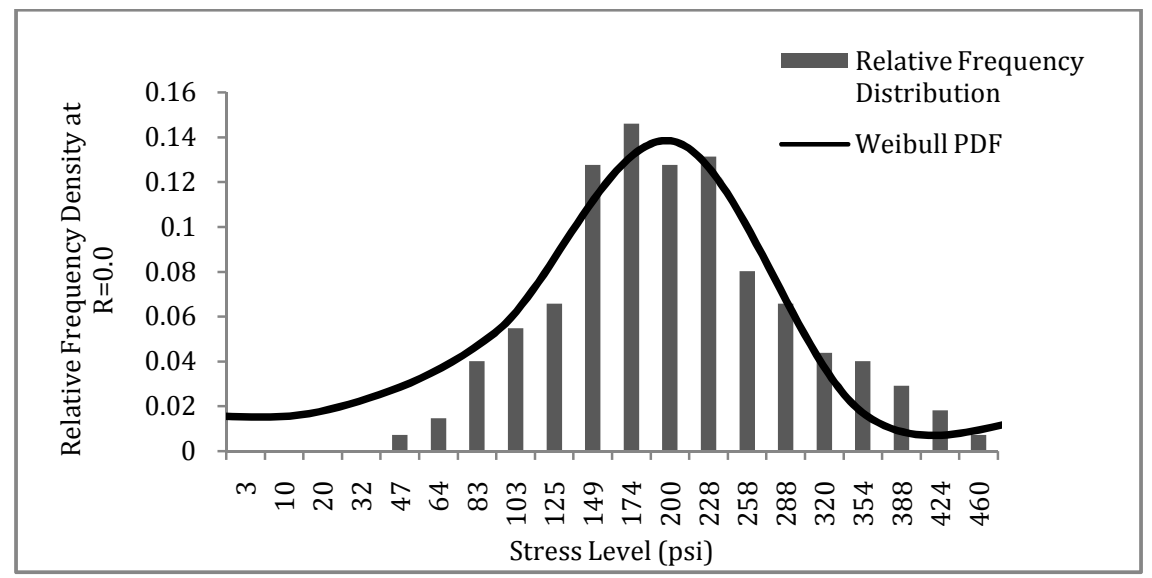

Figure 6-7. Weibull curve fit for stress ratio bin $\mathrm{R}=0.0$.

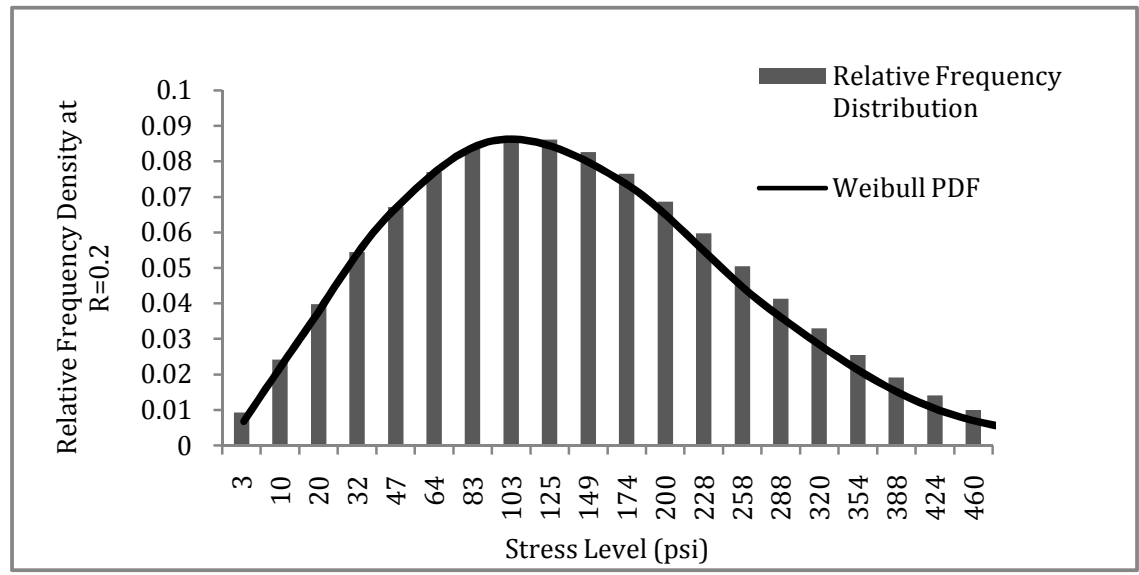

Figure 6-8. Weibull curve fit for stress ratio bin $\mathrm{R}=0.2$.

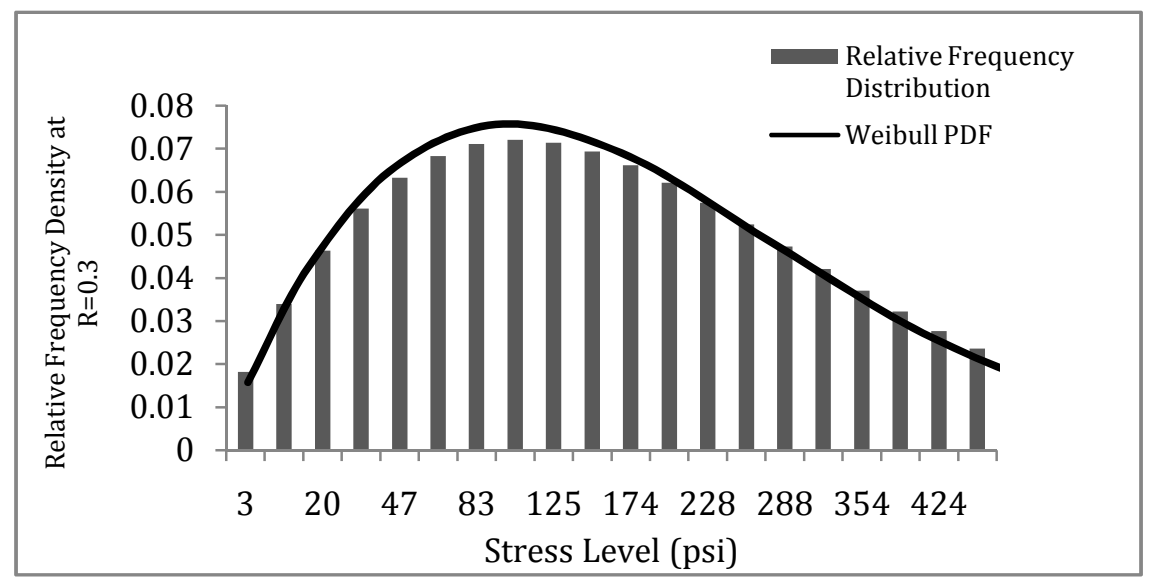

Figure 6-9. Weibull curve fit for stress ratio bin $\mathrm{R}=0.3$. 


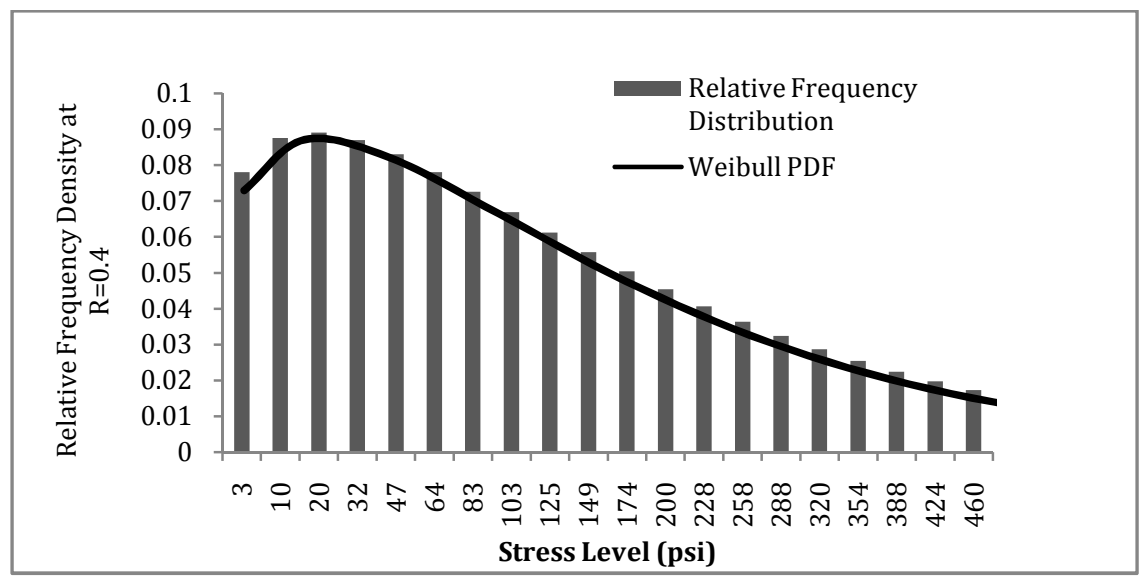

Figure 6-10. Weibull curve fit for stress ratio bin $\mathrm{R}=0.4$.

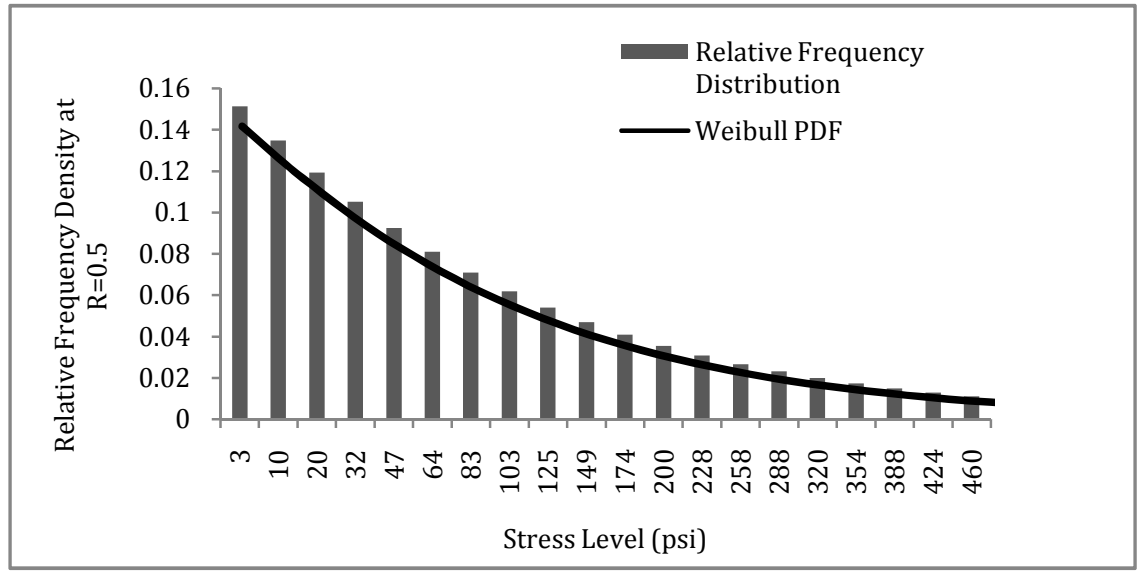

Figure 6-11. Weibull curve fit for stress ratio bin $\mathrm{R}=0.5$.

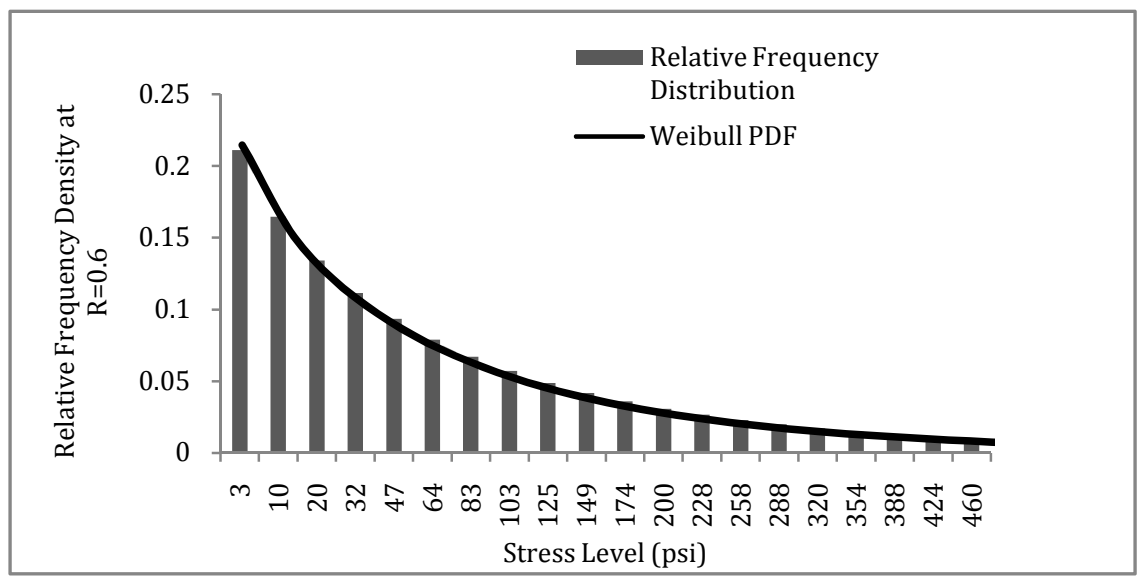

Figure 6-12. Weibull curve fit for stress ratio bin $\mathrm{R}=0.6$. 


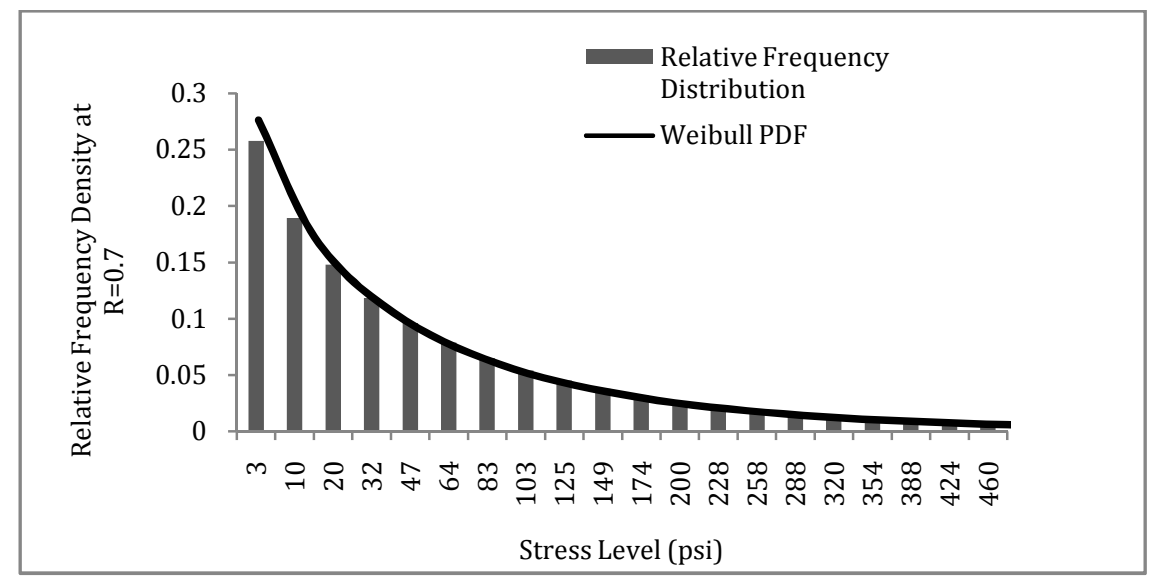

Figure 6-13. Weibull curve fit for stress ratio bin $\mathrm{R}=0.7$.

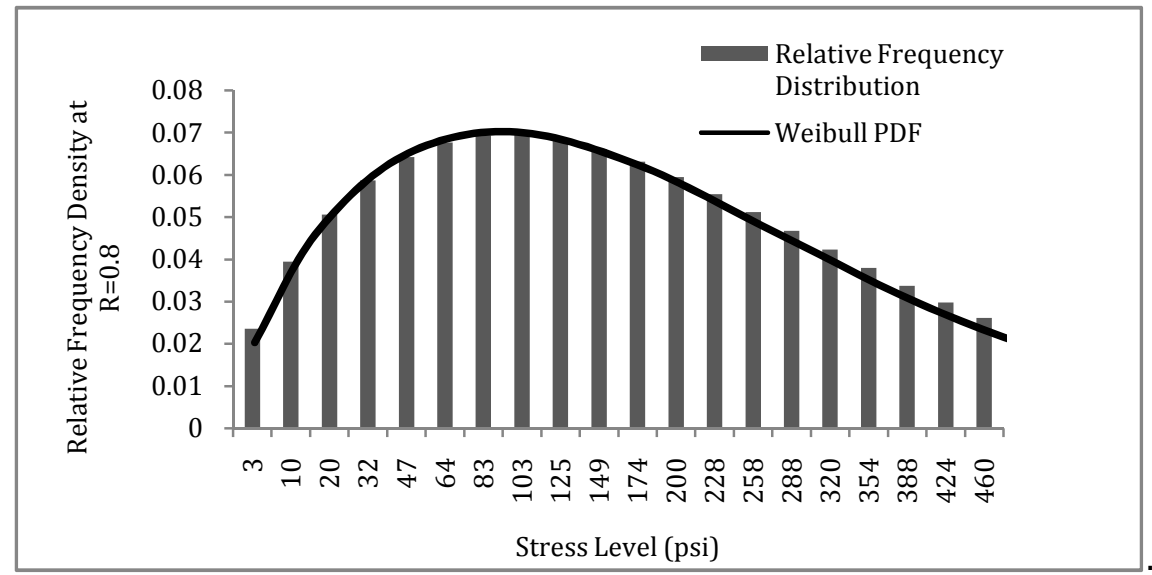

Figure 6-14. Weibull curve fit for stress ratio bin $\mathrm{R}=0.8$.

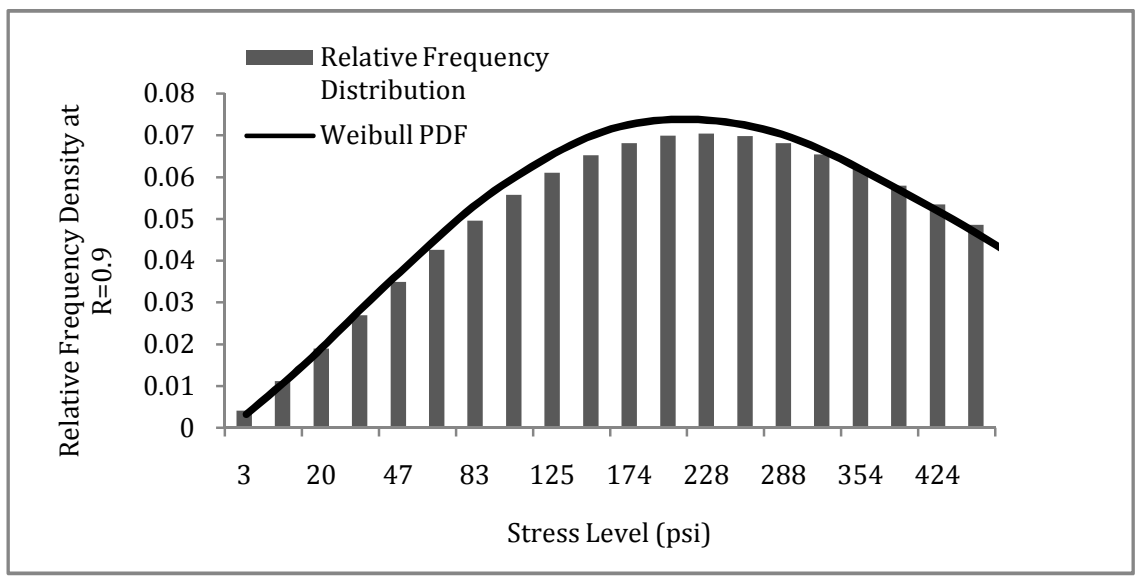

Figure 6-15. Weibull curve fit for stress ratio bin $\mathrm{R}=0.9$. 
Table 6-2. Cumulative damage totals based on fatigue loading spectrum for a 20 service life.

\begin{tabular}{|c|c|c|c|c|c|c|c|c|c|c|c|}
\hline \multirow{2}{*}{$\begin{array}{c}\text { Normalized } \\
\text { Stress }\end{array}$} & \multicolumn{10}{|c|}{ Stress Ratio Bin Center $\mathrm{R}=$ Min/Max stress } & \multirow{2}{*}{$\begin{array}{c}\text { Row } \\
\text { Totals }\end{array}$} \\
\hline & 0 & 0.1 & 0.2 & 0.3 & 0.4 & 0.5 & 0.6 & 0.7 & 0.8 & 0.9 & \\
\hline 0.01 & $6.4 \mathrm{E}-21$ & $2.1 \mathrm{E}-06$ & $2.9 \mathrm{E}-05$ & $1.2 \mathrm{E}-04$ & 7.7E-04 & $1.4 \mathrm{E}-03$ & $5.5 \mathrm{E}-03$ & 4.5E-03 & $9.5 \mathrm{E}-04$ & 7.2E-05 & $1.3 \mathrm{E}-02$ \\
\hline 0.02 & $1.9 \mathrm{E}-18$ & $4.2 \mathrm{E}-06$ & 5.0E-05 & 1.7E-04 & $8.1 \mathrm{E}-04$ & $1.3 \mathrm{E}-03$ & 4.7E-03 & $3.7 \mathrm{E}-03$ & $1.3 \mathrm{E}-03$ & $1.3 \mathrm{E}-04$ & $1.2 \mathrm{E}-02$ \\
\hline 0.04 & $9.4 \mathrm{E}-17$ & $6.8 \mathrm{E}-06$ & 7.2E-05 & $2.1 \mathrm{E}-04$ & $8.4 \mathrm{E}-04$ & $1.2 \mathrm{E}-03$ & 4.1E-03 & $3.1 \mathrm{E}-03$ & $1.6 \mathrm{E}-03$ & $1.9 \mathrm{E}-04$ & 1.1E-02 \\
\hline 0.06 & $1.9 \mathrm{E}-15$ & 9.7E-06 & $9.5 \mathrm{E}-05$ & $2.6 \mathrm{E}-04$ & $8.6 \mathrm{E}-04$ & $1.2 \mathrm{E}-03$ & 3.7E-03 & 2.7E-03 & $1.8 \mathrm{E}-03$ & 2.7E-04 & $1.1 \mathrm{E}-02$ \\
\hline 0.09 & $2.3 \mathrm{E}-14$ & $1.3 \mathrm{E}-05$ & $1.2 \mathrm{E}-04$ & 3.0E-04 & 8.7E-04 & $1.1 \mathrm{E}-03$ & $3.3 \mathrm{E}-03$ & $2.4 \mathrm{E}-03$ & $2.1 \mathrm{E}-03$ & 3.5E-04 & 1.1E-02 \\
\hline 0.12 & $1.8 \mathrm{E}-13$ & 1.7E-05 & $1.4 \mathrm{E}-04$ & $3.4 \mathrm{E}-04$ & $8.8 \mathrm{E}-04$ & $1.1 \mathrm{E}-03$ & $3.1 \mathrm{E}-03$ & $2.1 \mathrm{E}-03$ & $2.3 \mathrm{E}-03$ & 4.4E-04 & 1.0E-02 \\
\hline 0.16 & $1.1 \mathrm{E}-12$ & $2.1 \mathrm{E}-05$ & $1.6 \mathrm{E}-04$ & 3.7E-04 & $8.9 \mathrm{E}-04$ & $1.0 \mathrm{E}-03$ & $2.9 \mathrm{E}-03$ & $1.9 \mathrm{E}-03$ & $2.5 \mathrm{E}-03$ & $5.5 \mathrm{E}-04$ & $1.0 \mathrm{E}-02$ \\
\hline 0.25 & $2.5 \mathrm{E}-11$ & $2.8 \mathrm{E}-05$ & $1.8 \mathrm{E}-04$ & 4.4E-04 & $9.0 \mathrm{E}-04$ & 9.3E-04 & $2.6 \mathrm{E}-03$ & 1.7E-03 & 2.9E-03 & $7.8 \mathrm{E}-04$ & $1.0 \mathrm{E}-02$ \\
\hline 0.30 & $9.4 \mathrm{E}-11$ & 3.1E-05 & $1.9 \mathrm{E}-04$ & 4.7E-04 & $9.1 \mathrm{E}-04$ & 9.1E-04 & $2.5 \mathrm{E}-03$ & $1.6 \mathrm{E}-03$ & 3.1E-03 & $9.1 \mathrm{E}-04$ & $1.1 \mathrm{E}-02$ \\
\hline 0.36 & $3.2 \mathrm{E}-10$ & $3.4 \mathrm{E}-05$ & $1.9 \mathrm{E}-04$ & 5.0E-04 & $9.2 \mathrm{E}-04$ & 8.9E-04 & $2.4 \mathrm{E}-03$ & $1.5 \mathrm{E}-03$ & $3.3 \mathrm{E}-03$ & $1.1 \mathrm{E}-03$ & $1.1 \mathrm{E}-02$ \\
\hline 0.42 & 1.0E-09 & 3.7E-05 & $1.9 \mathrm{E}-04$ & 5.2E-04 & $9.4 \mathrm{E}-04$ & 8.7E-04 & $2.3 \mathrm{E}-03$ & $1.4 \mathrm{E}-03$ & $3.5 \mathrm{E}-03$ & $1.2 \mathrm{E}-03$ & $1.1 \mathrm{E}-02$ \\
\hline 0.49 & 3.0E-09 & $3.8 \mathrm{E}-05$ & $1.8 \mathrm{E}-04$ & 5.4E-04 & $9.6 \mathrm{E}-04$ & 8.6E-04 & $2.3 \mathrm{E}-03$ & $1.4 \mathrm{E}-03$ & 3.7E-03 & $1.4 \mathrm{E}-03$ & $1.1 \mathrm{E}-02$ \\
\hline 0.56 & 8.2E-09 & $3.9 E-05$ & 1.7E-04 & $5.6 \mathrm{E}-04$ & $9.7 \mathrm{E}-04$ & 8.5E-04 & $2.3 \mathrm{E}-03$ & 1.3E-03 & $3.9 \mathrm{E}-03$ & $1.5 \mathrm{E}-03$ & $1.2 \mathrm{E}-02$ \\
\hline 0.64 & $2.2 \mathrm{E}-08$ & $3.8 \mathrm{E}-05$ & $1.6 \mathrm{E}-04$ & 5.7E-04 & 1.0E-03 & 8.5E-04 & $2.3 \mathrm{E}-03$ & $1.3 \mathrm{E}-03$ & 4.0E-03 & 1.7E-03 & $1.2 \mathrm{E}-02$ \\
\hline 0.72 & $5.4 \mathrm{E}-08$ & 3.7E-05 & $1.4 \mathrm{E}-04$ & $5.8 \mathrm{E}-04$ & $1.0 \mathrm{E}-03$ & 8.5E-04 & $2.3 \mathrm{E}-03$ & $1.3 \mathrm{E}-03$ & 4.2E-03 & $1.8 \mathrm{E}-03$ & $1.2 \mathrm{E}-02$ \\
\hline 0.81 & $1.3 \mathrm{E}-07$ & 3.5E-05 & $1.2 \mathrm{E}-04$ & 5.9E-04 & 1.1E-03 & 8.6E-04 & $2.3 \mathrm{E}-03$ & 1.3E-03 & 4.3E-03 & 2.0E-03 & 1.3E-02 \\
\hline 1.00 & $6.8 \mathrm{E}-07$ & 2.9E-05 & 8.9E-05 & 6.0E-04 & $1.1 \mathrm{E}-03$ & $8.8 \mathrm{E}-04$ & $2.4 \mathrm{E}-03$ & $1.3 \mathrm{E}-03$ & 4.6E-03 & $2.3 \mathrm{E}-03$ & 1.3E-02 \\
\hline
\end{tabular}



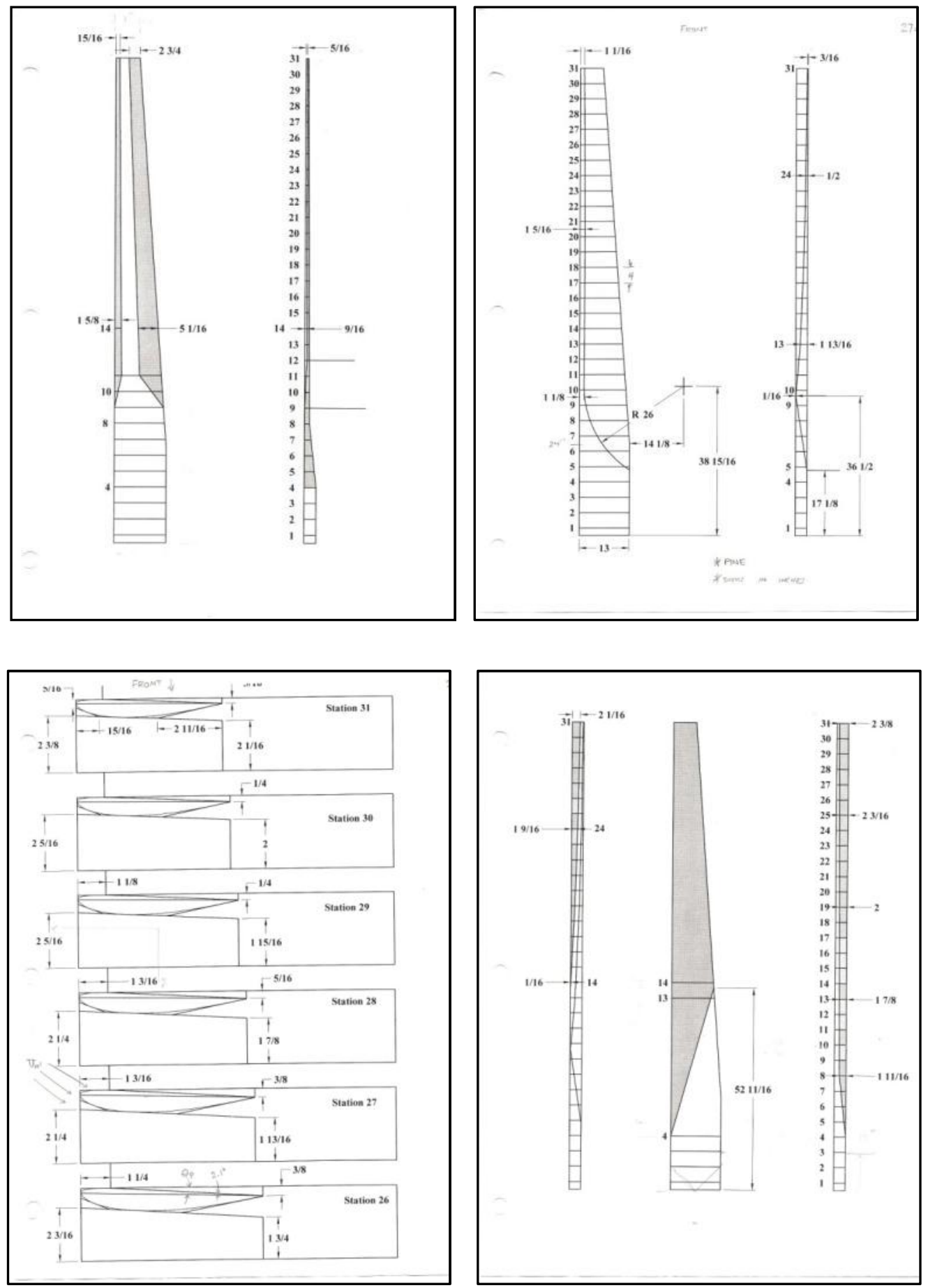

Figure 6-16. Blade profile design as provided by Gorman Controls, Ltd. 


\section{Chapter 4 Figures and Tables}

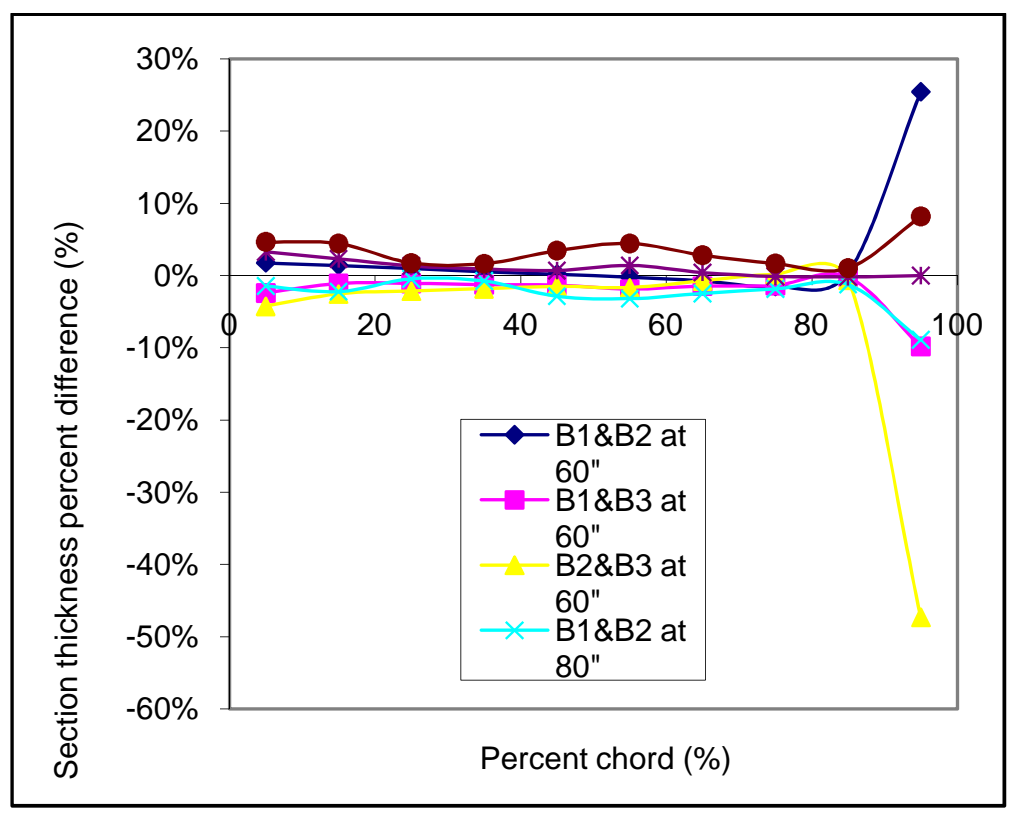

Figure 6-17. Comparison of chord length between all three blades at sections located 60 inches and 80 inches from the root

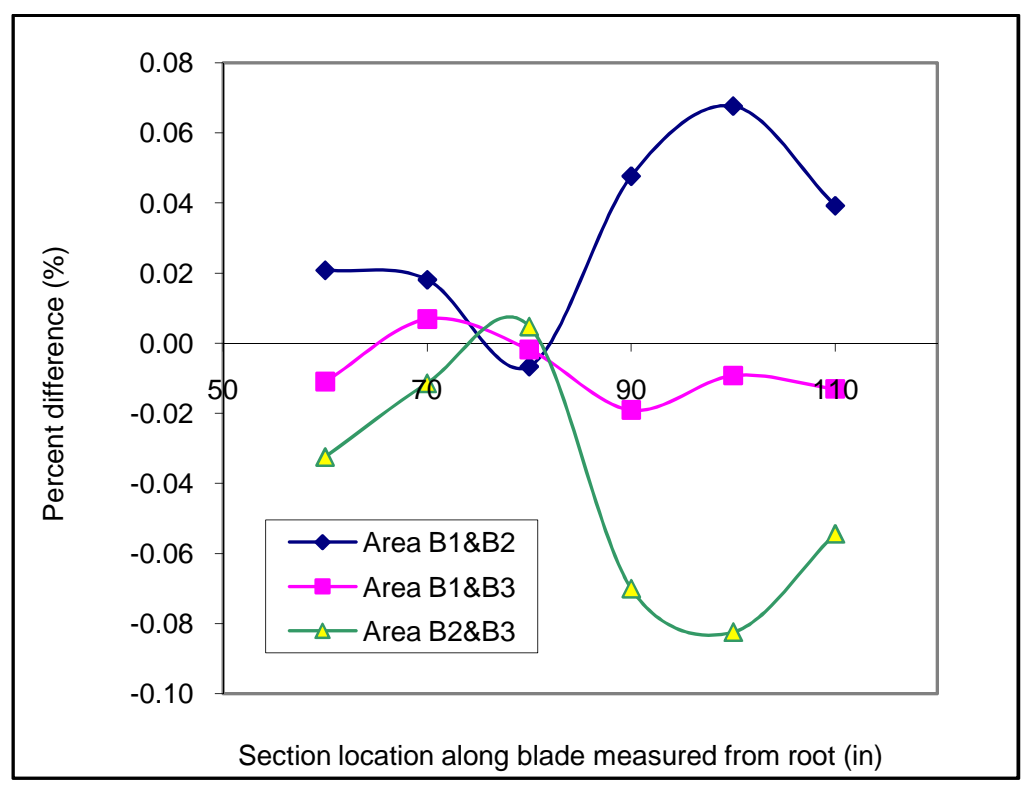

Figure 6-18. Measured cross sectional area variation between all three blades. 


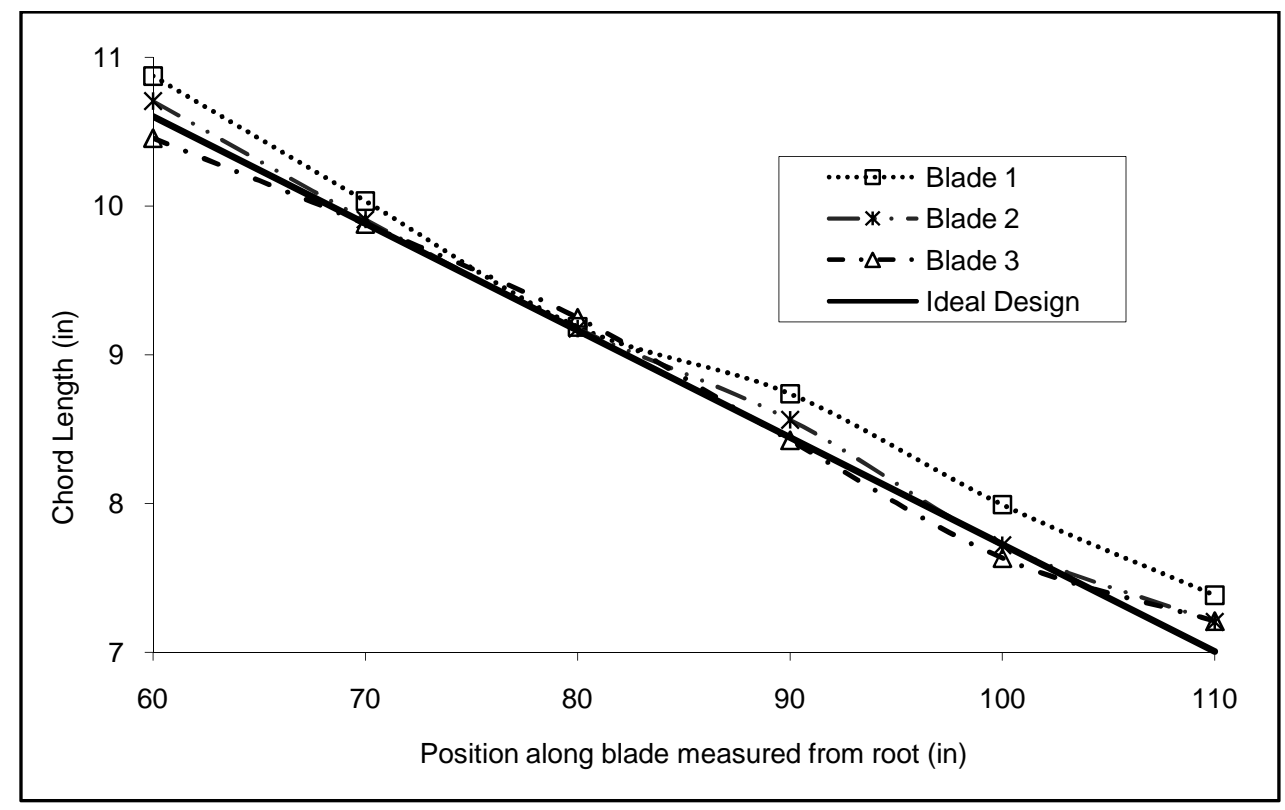

Figure 6-19. Chord length variation as compared to the ideal designed chord length for all three blades.

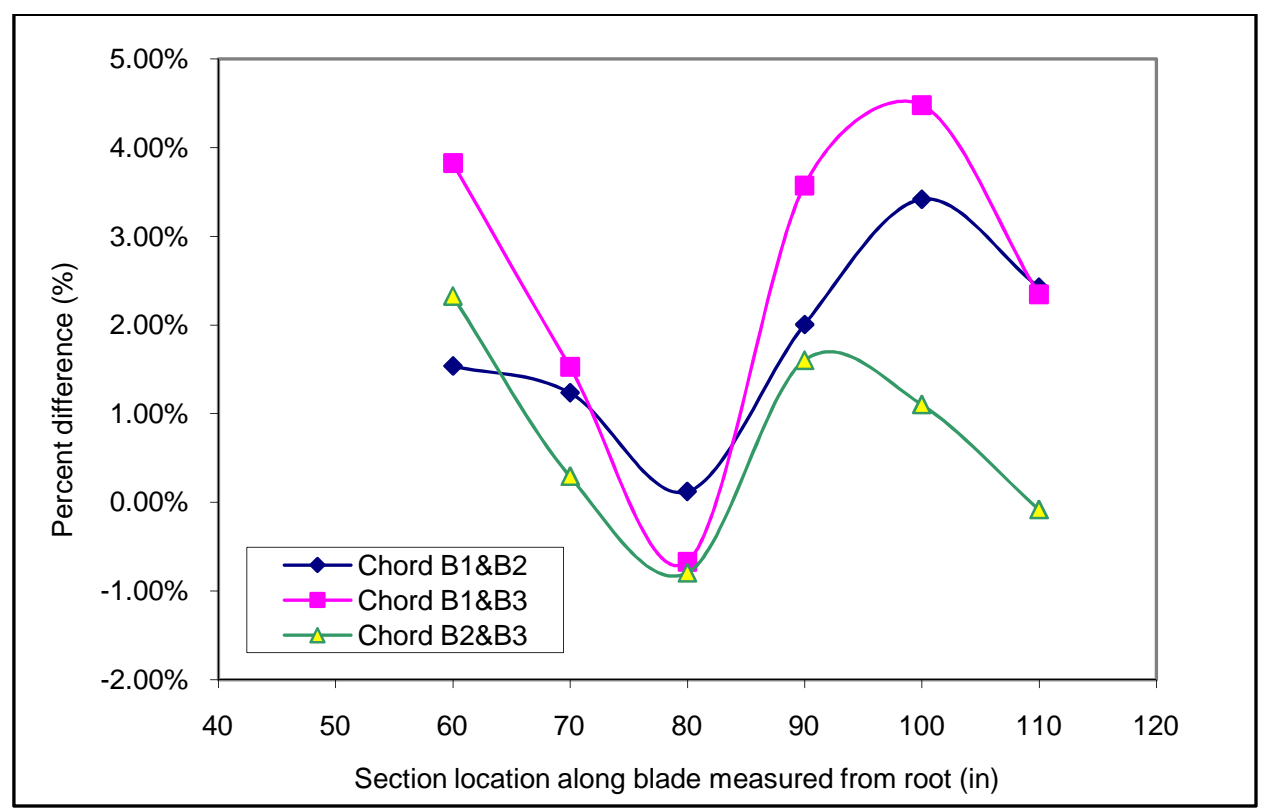

Figure 6-20. Chord length variance as a function of radial position for all three blades compared to one another. 


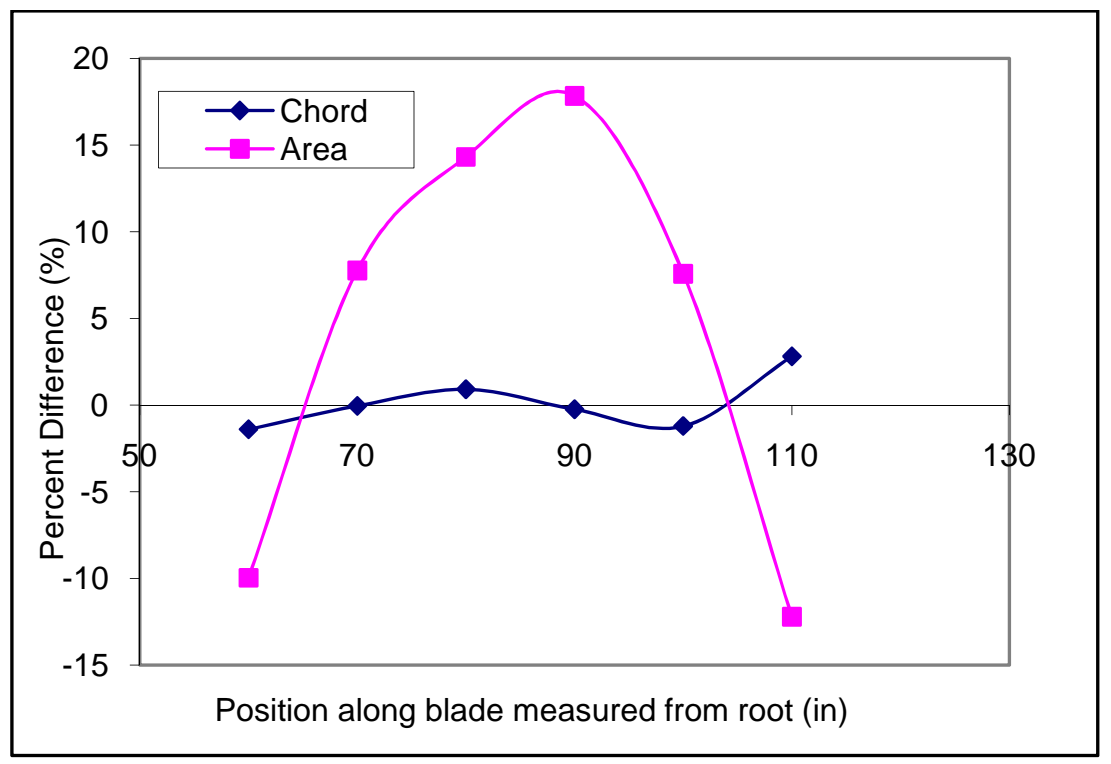

Figure 6-21. Chord length and cross sectional area variance compared to the ideal Clark Y profile for blade $\mathrm{C}$.

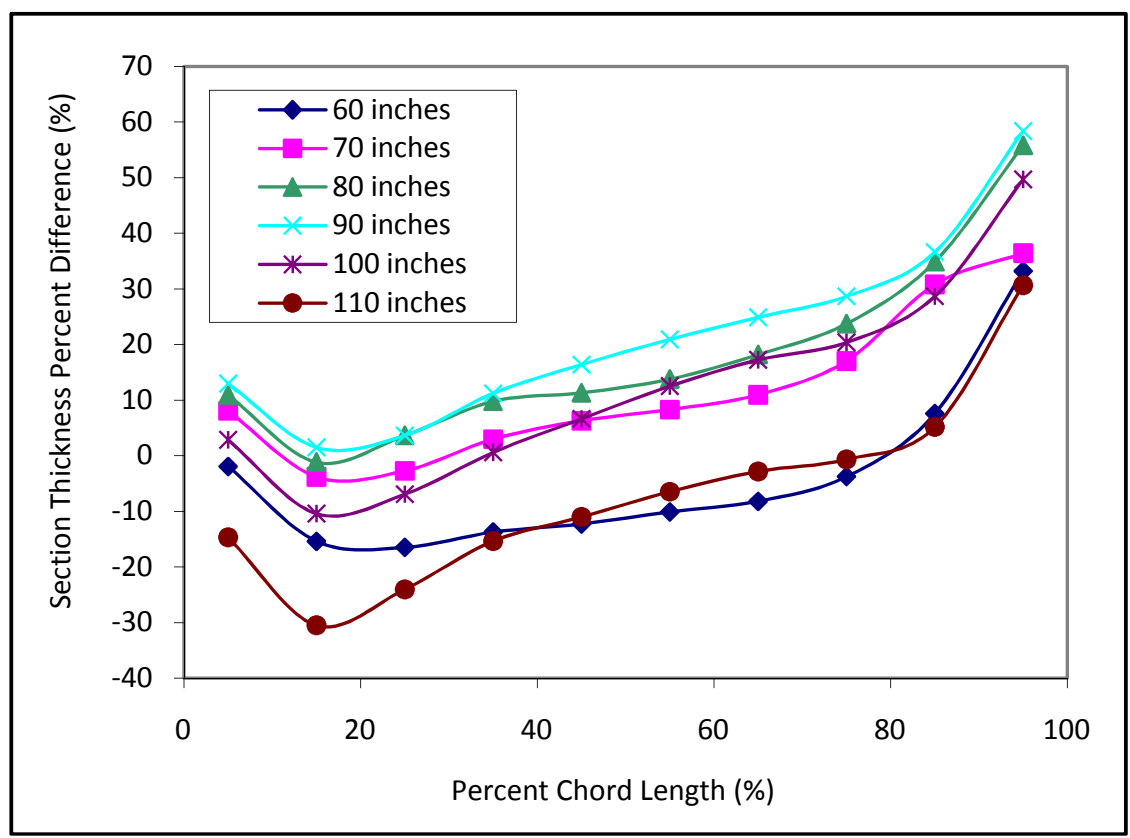

Figure 6-22. Section thickness variation compared to the ideal Clark Y profile as a function of chord length in inches for blade C. Six profiles were measured in all. 


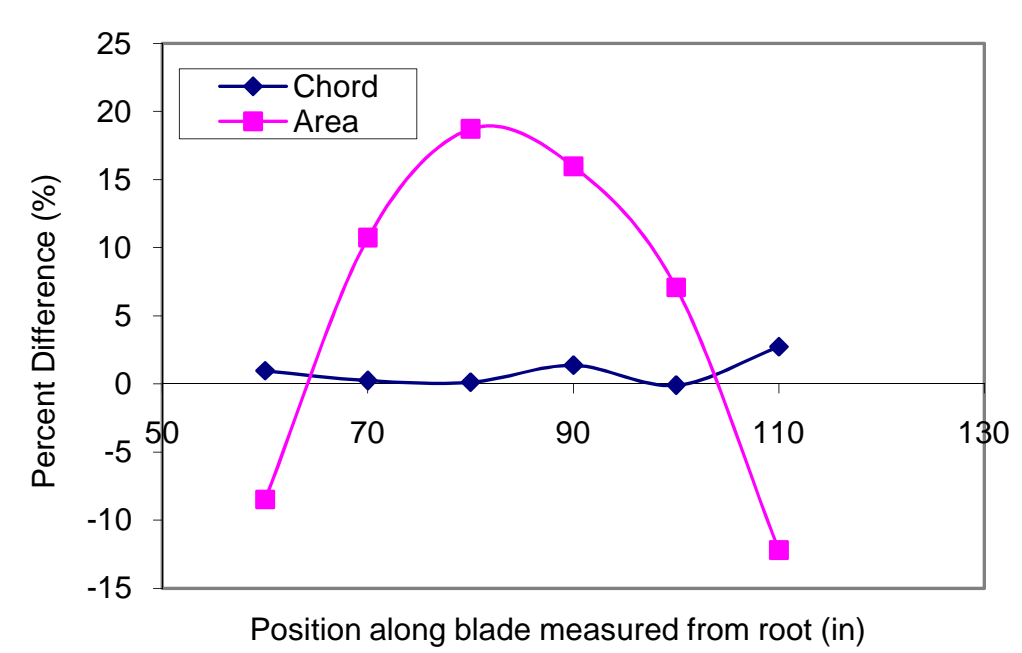

Figure 6-23. Chord length and cross sectional area variance compared to the ideal Clark Y profile for blade B

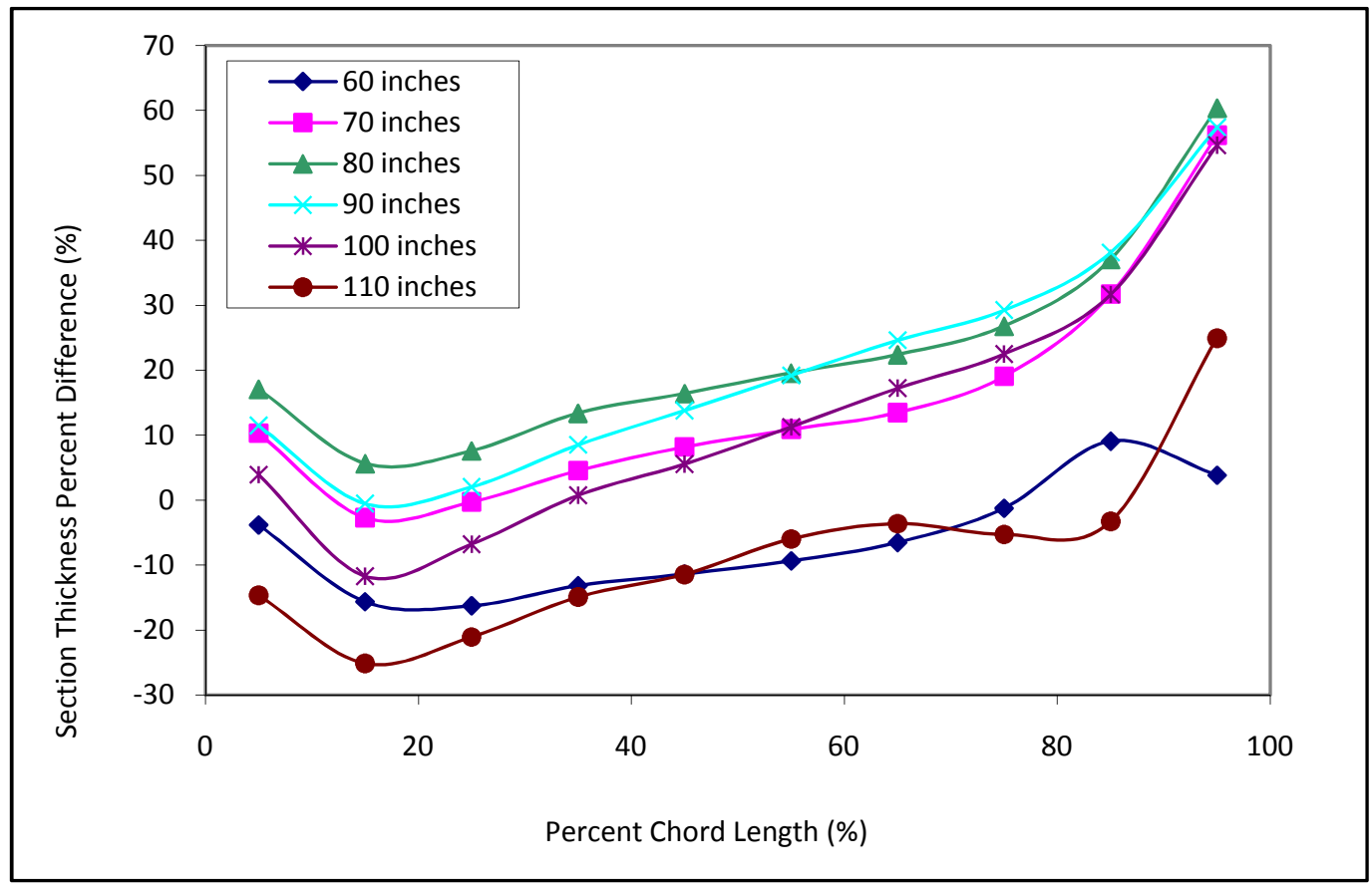

Figure 6-24. Section thickness variation compared to the ideal Clark Y profile as a function of chord length in inches for blade B. Six profiles along the blade were measured in all. 


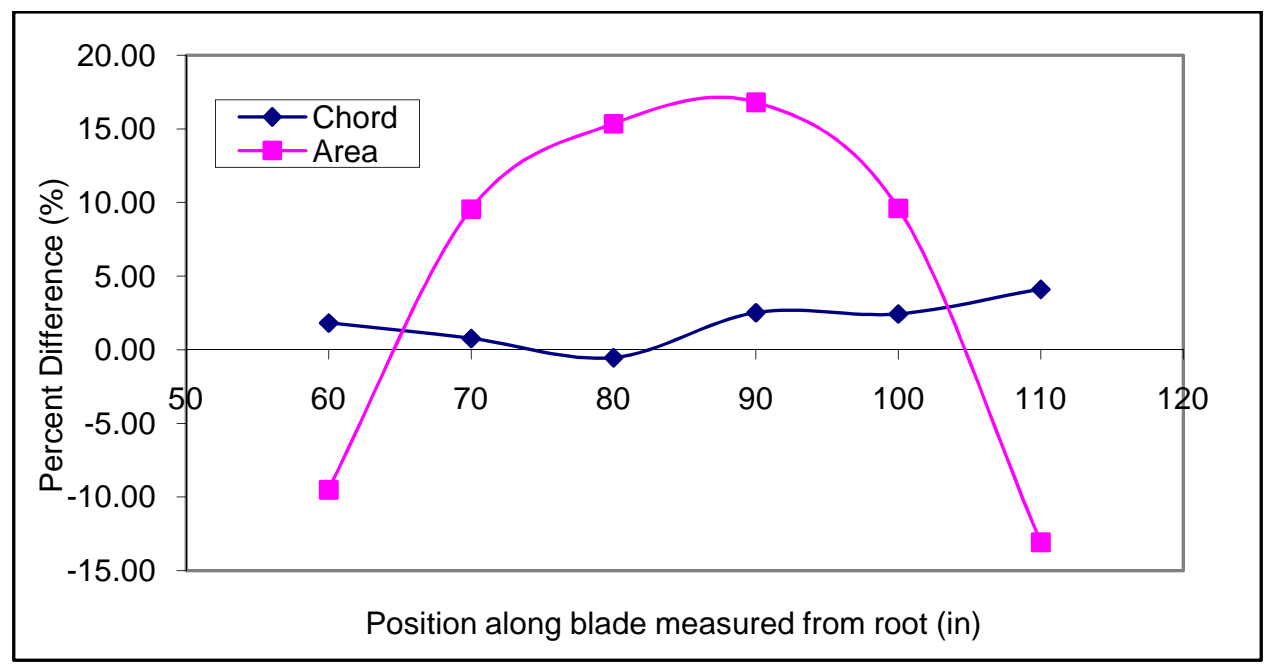

Figure 6-25. Chord length and cross sectional area variance compared to the ideal Clark Y profile for blade A

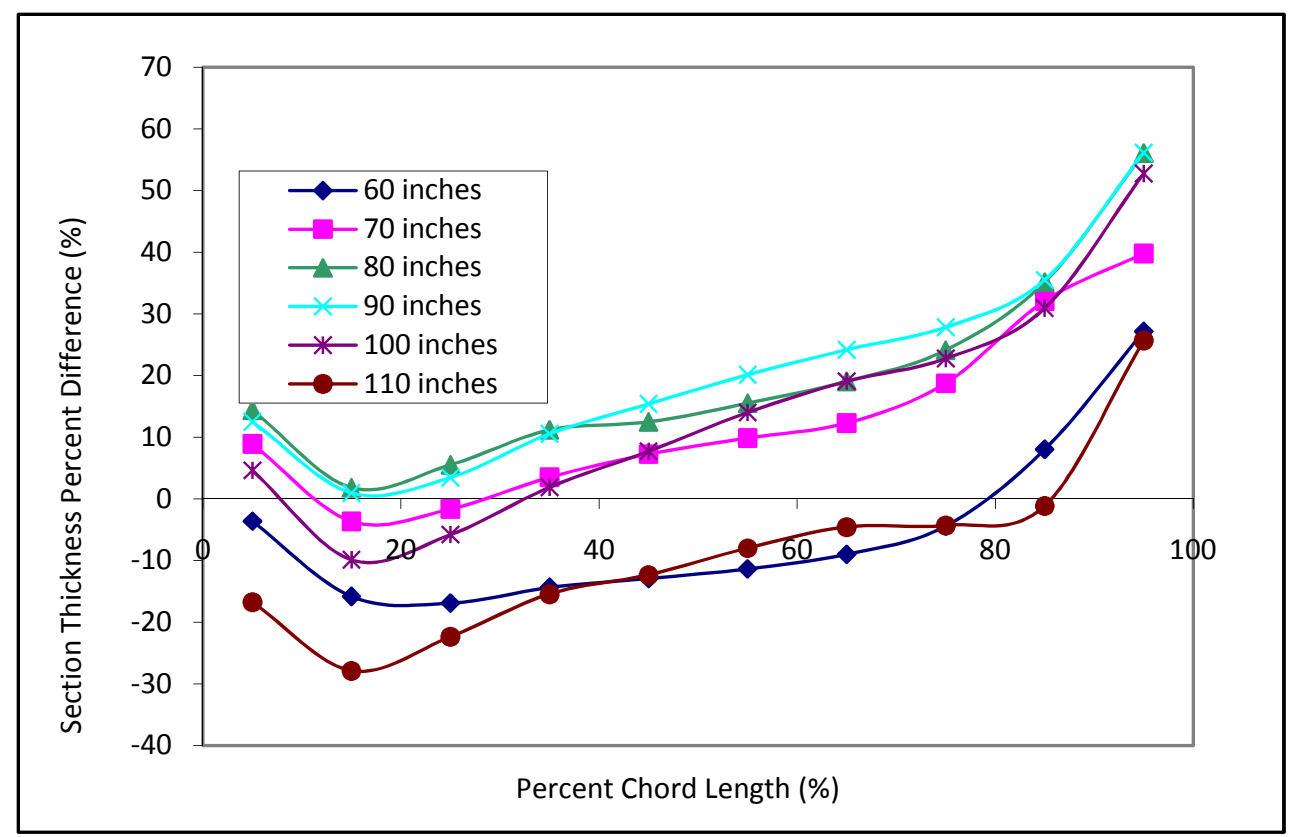

Figure 6-26. Section thickness variation compared to the ideal Clark Y profile as a function of chord length in inches for blade A. Six profiles along the blade were measured in all. 


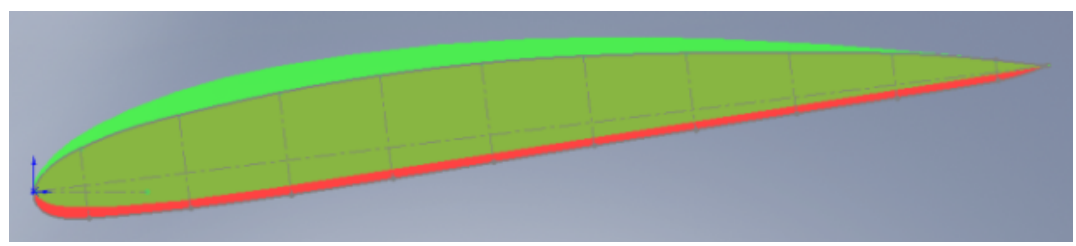

Blade A - Section at 60", Chord=10.872"

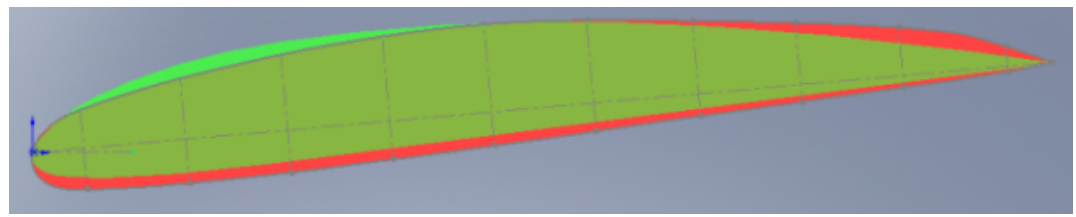

Blade A - Section at 70", Chord=10.032

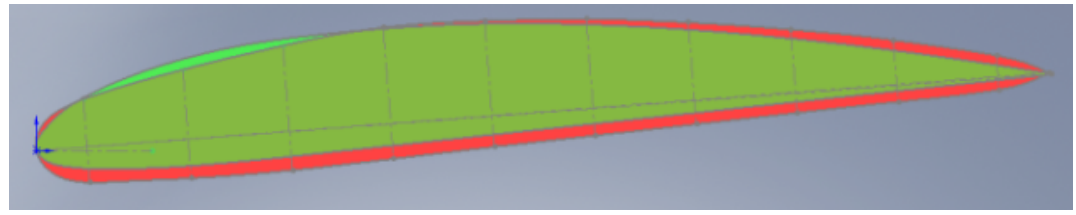

Blade A - Section at 80", Chord=9.186"

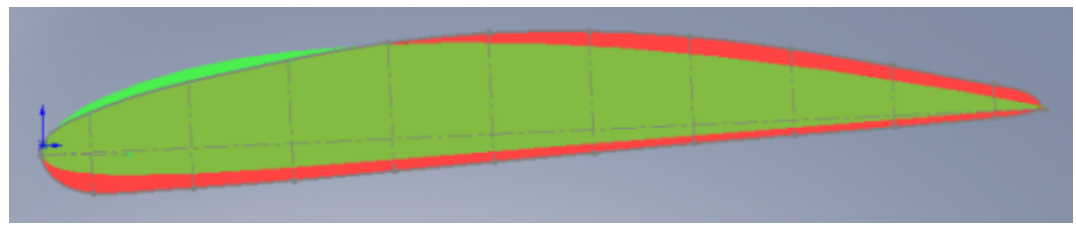

Blade A - Section at 90", Chord=8.737"

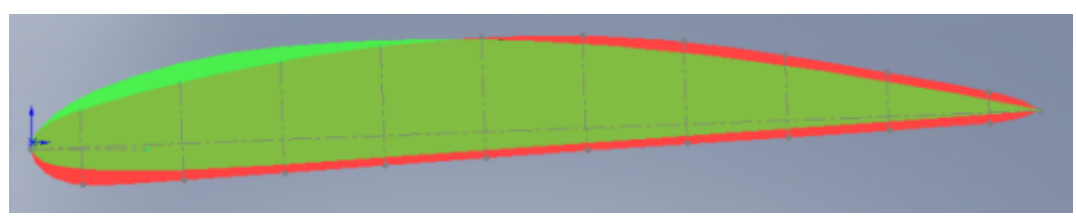

Blade A - Section at 100", Chord=7.992"

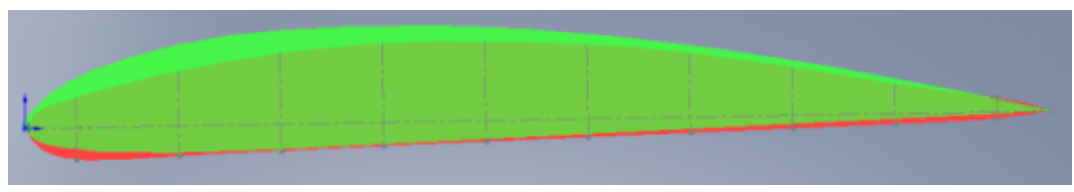

Blade A - Section at 110", Chord=7.383"

Figure 6-27. Illustration of the six section profiles for blade A used in comparison to ideal Clark Y airfoil shape. Green is ideal Clark Y and red is the measured profile. 


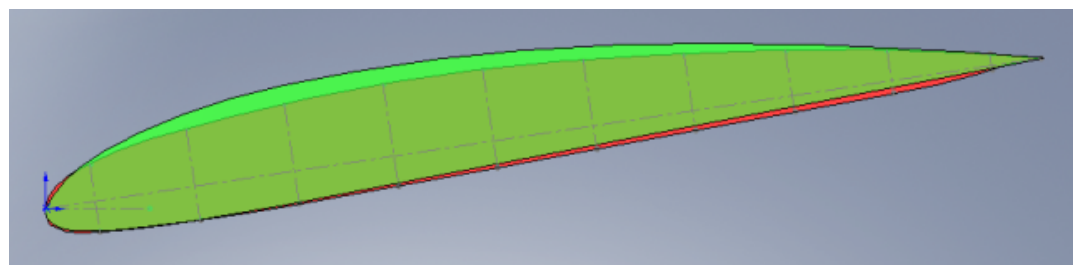

Blade B - Section at 60" Chord=10.705"

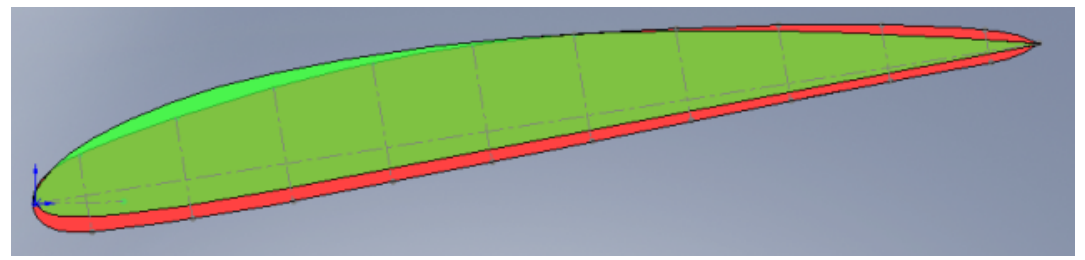

Blade B - Section at 70", Chord=9.868"

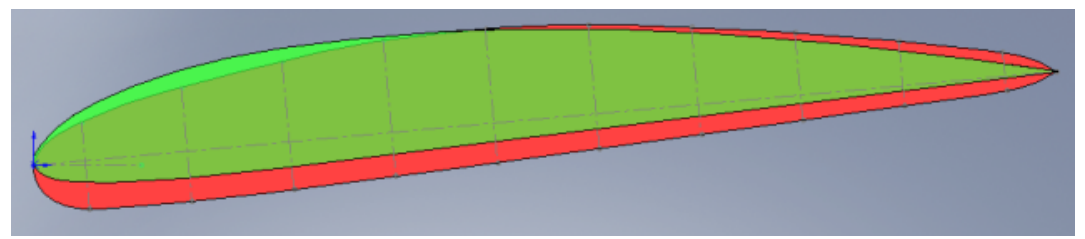

Blade B - Section at 80", Chord=9.028

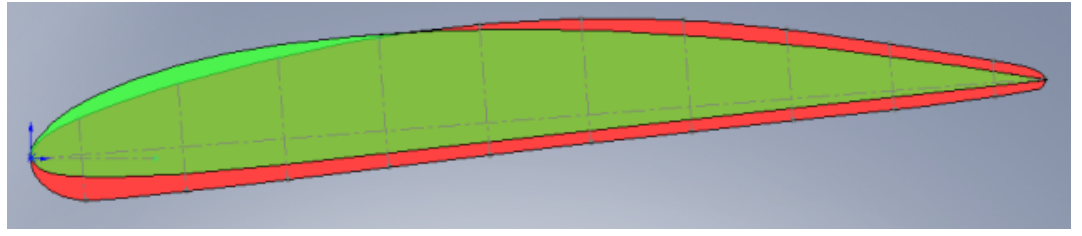

Blade B - Section at 90", Chord=8.567

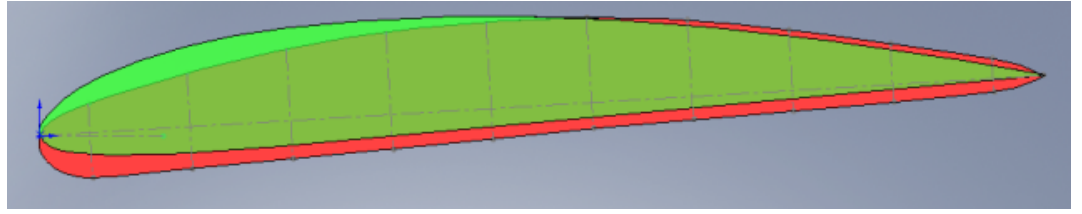

Blade B - Section at 100", Chord=7.821

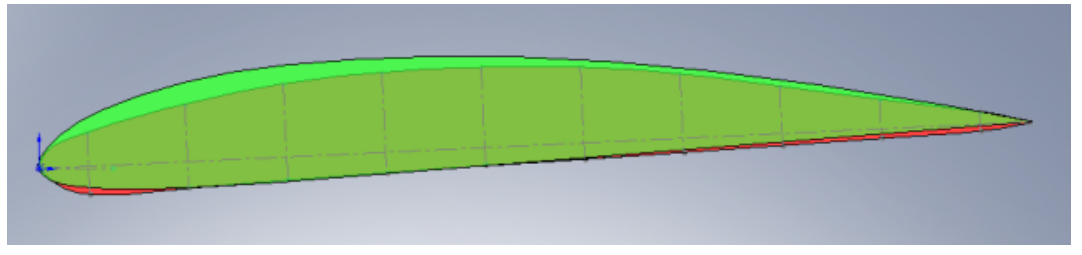

Blade B - Section at 110" Chord=7.204

Figure 6-28. Illustration of the six section profiles for blade B used in comparison with the ideal Clark Y airfoil shape. Green is ideal Clark Y and red is the measured profile. 


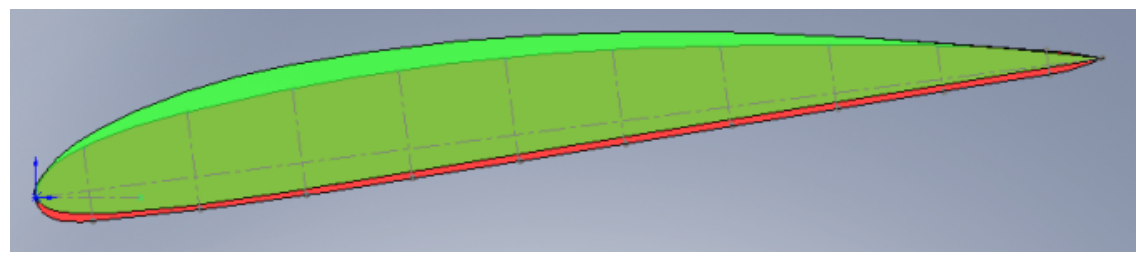

Blade C - Section at 60", Chord=10.952"

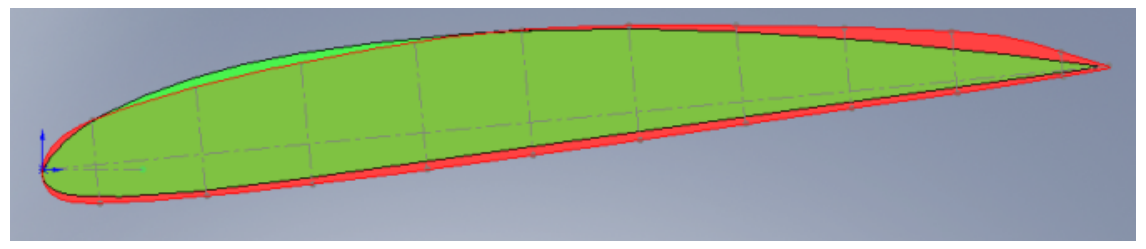

Blade C - Section at 70", Chord=10.088"

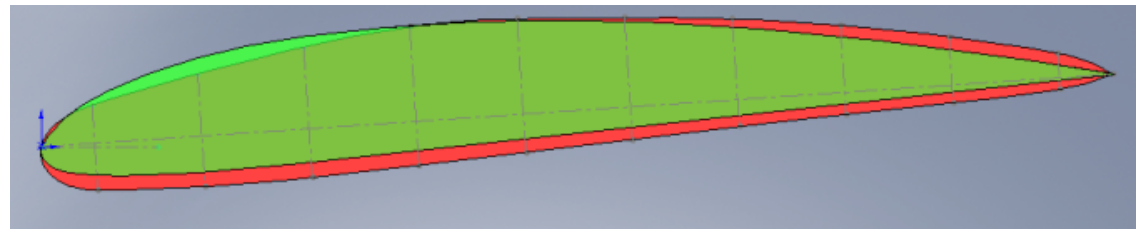

Blade C - Section at 80", Chord=9.248"

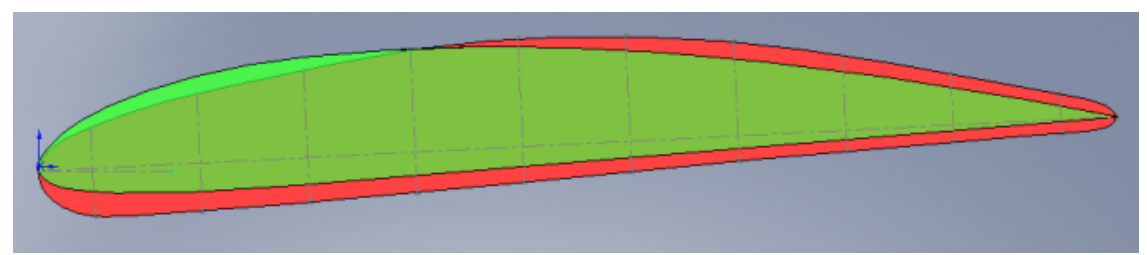

Blade C - Section at 90", Chord=8.764"

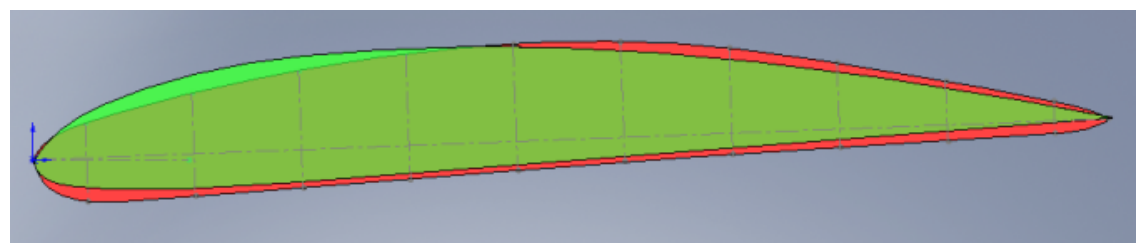

Blade C - Section at 100", Chord=8.116"

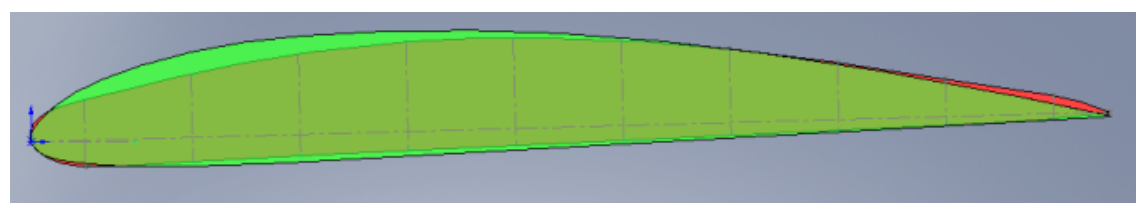

Blade C - Section at 110", Chord=7.398"

Figure 6-29.. Illustration of the six section profiles for blade $\mathrm{C}$ used in comparison with the ideal Clark Y airfoil shape. Green is ideal Clark Y and red is the measured profile. 


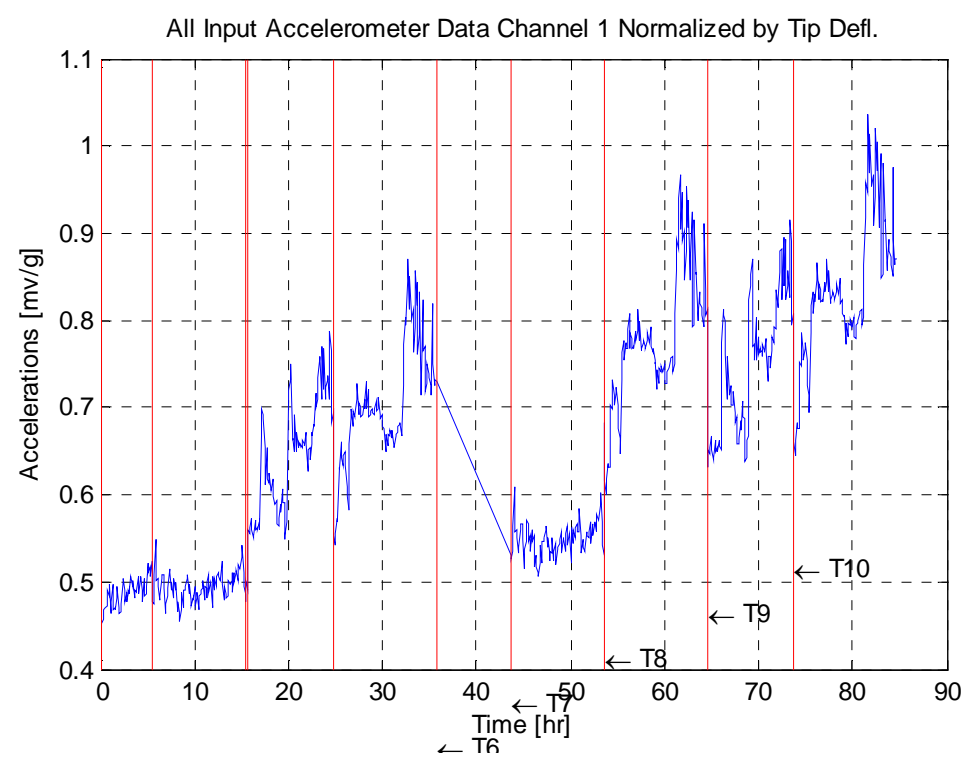

Figure 6-30. All input accelerometer data for full scale fatigue test performed on blade A. Each test is shown with a red vertical line and a T\# indicator.

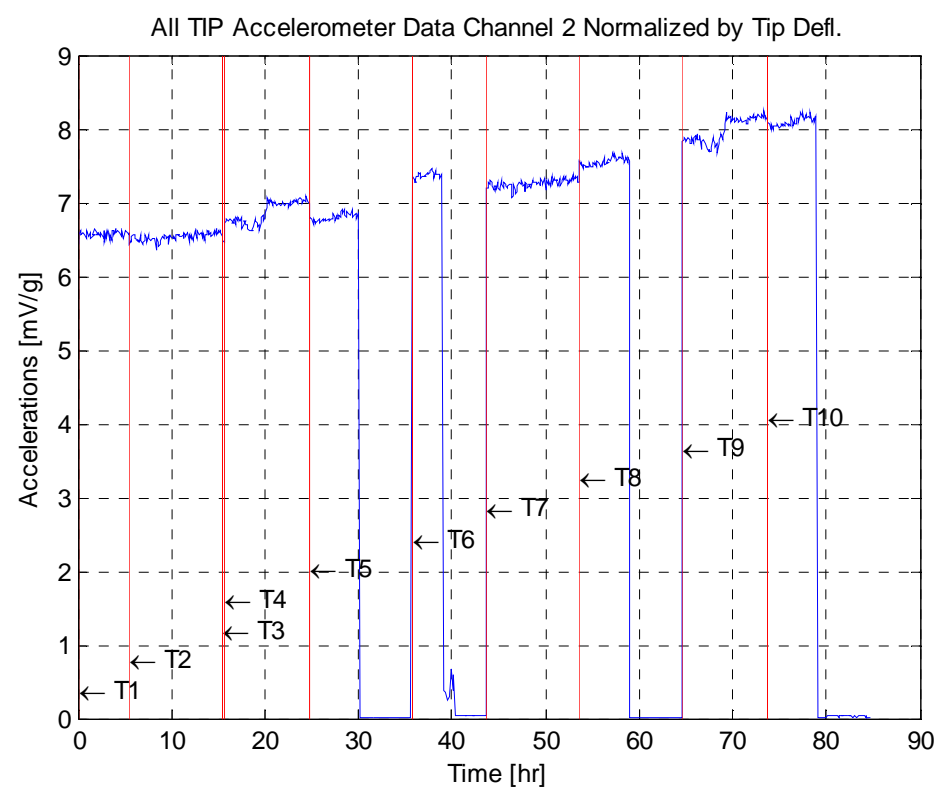

Figure 6-31. All tip accelerometer data for full scale fatigue test performed on blade A. Each test is shown with a red vertical line and a T\# indicator. 


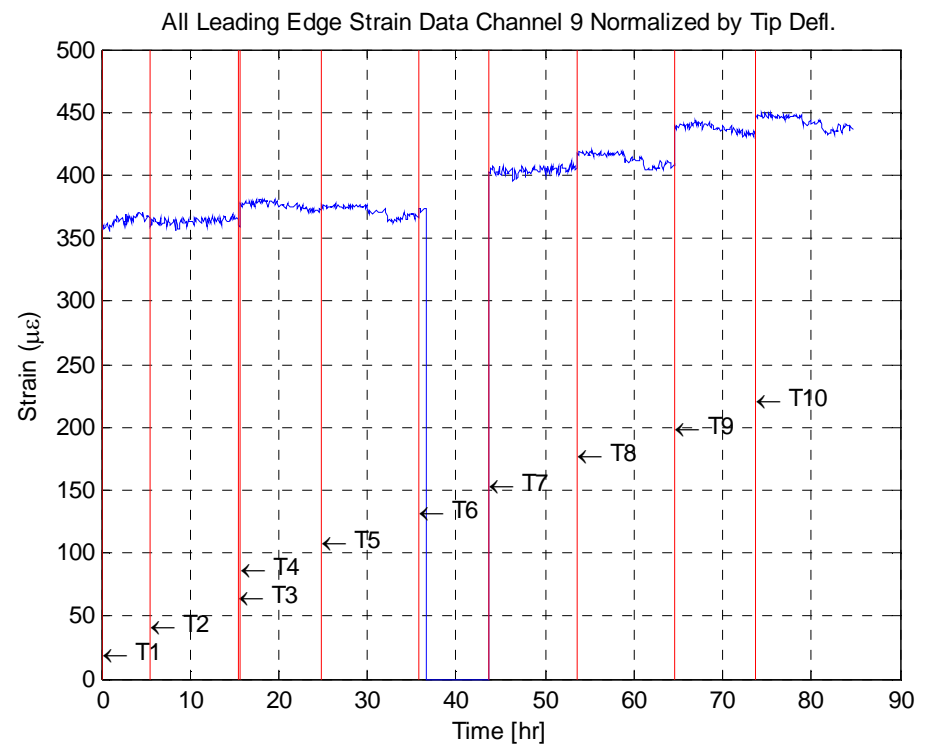

Figure 6-32. All leading edge strain gage data for full scale fatigue test performed on blade A. Each test is shown with a red vertical line and a T\# indicator.

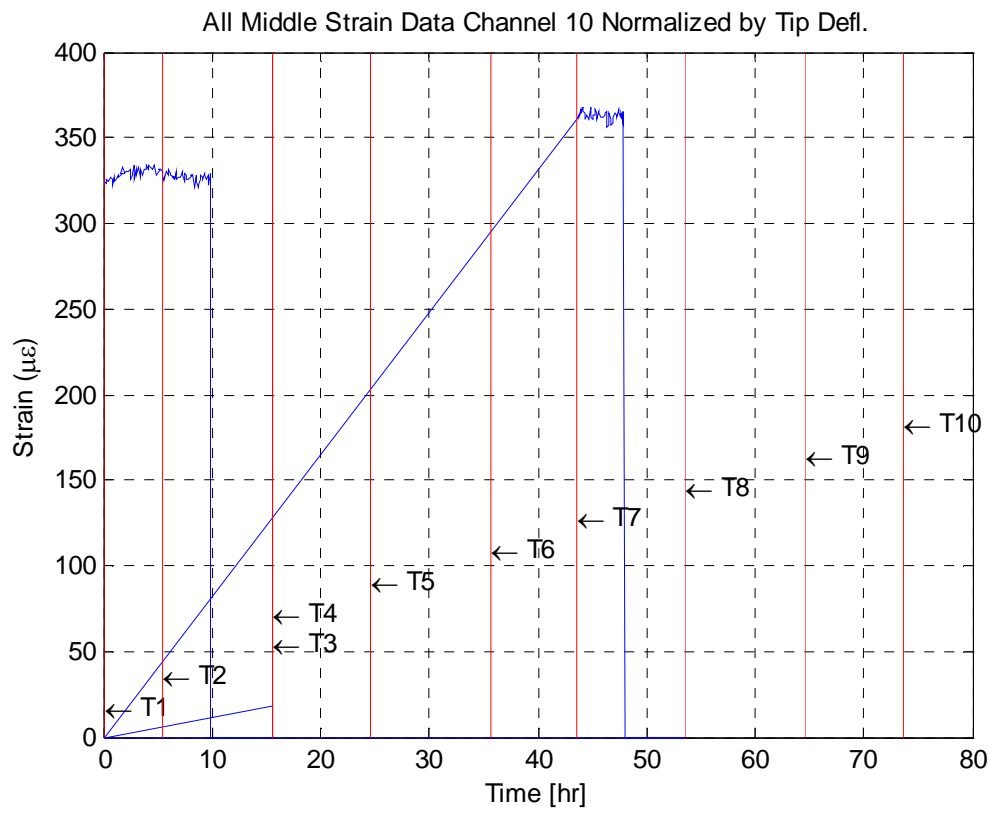

Figure 6-33. All middle strain gage data for full scale fatigue test performed on blade A. Each test is shown with a red vertical line and a $\mathrm{T} \#$ indicator. 


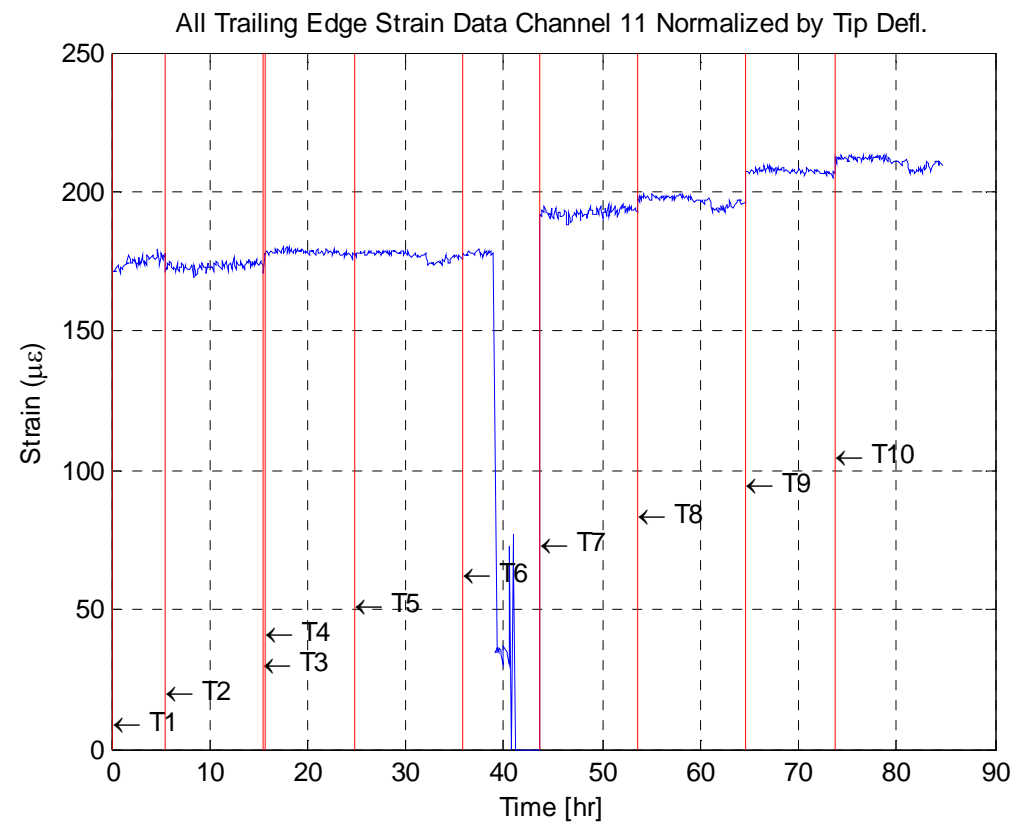

Figure 6-34. All trailing edge strain gage data for full scale fatigue test performed on blade A. Each test is shown with a red vertical line and a T\# indicator.

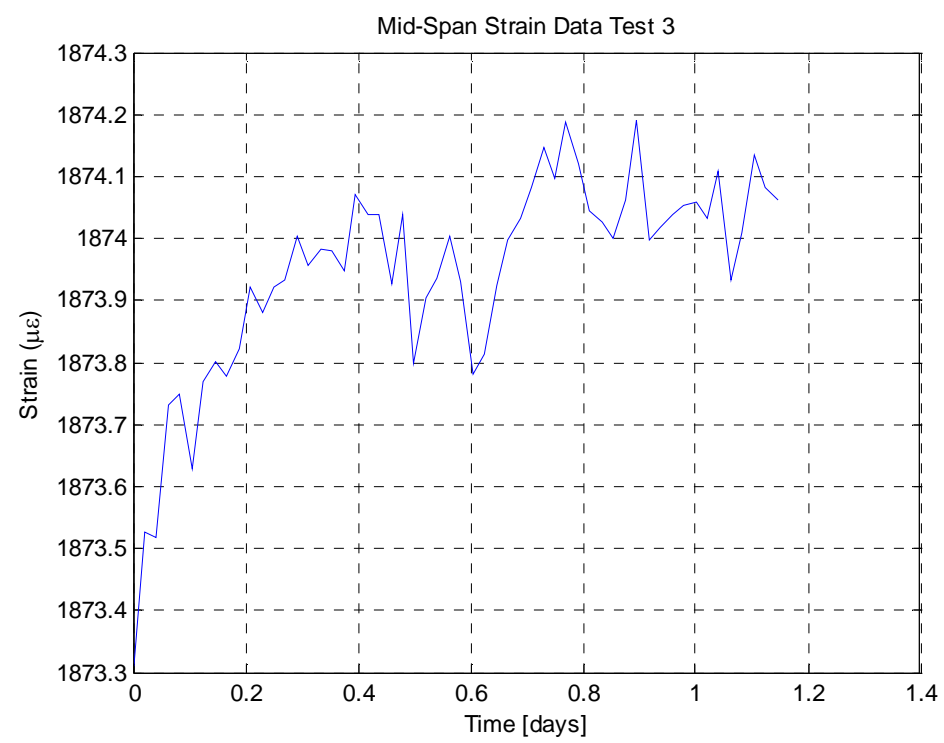

Figure 6-35. Strain gage output for the mid-span gages pair during test number three for blade B. 


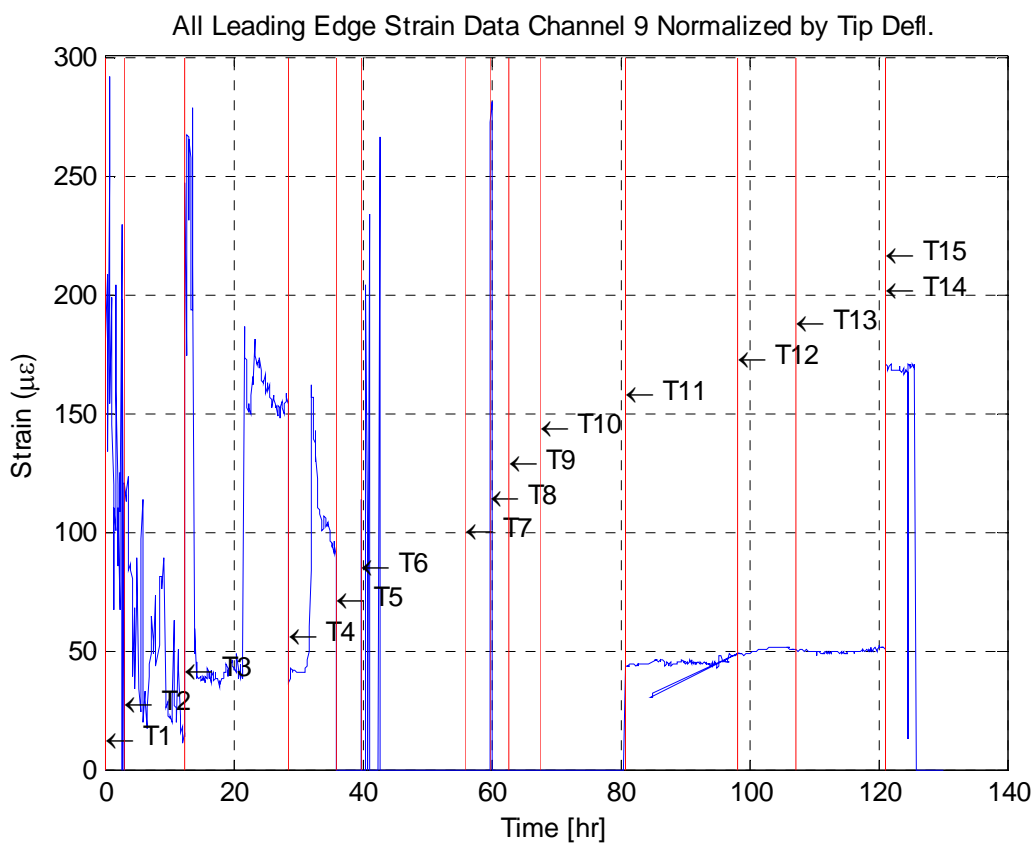

Figure 6-36. All leading edge strain gage data for full scale fatigue test performed on blade B. Each test is shown with a red vertical line and a T\# indicator.

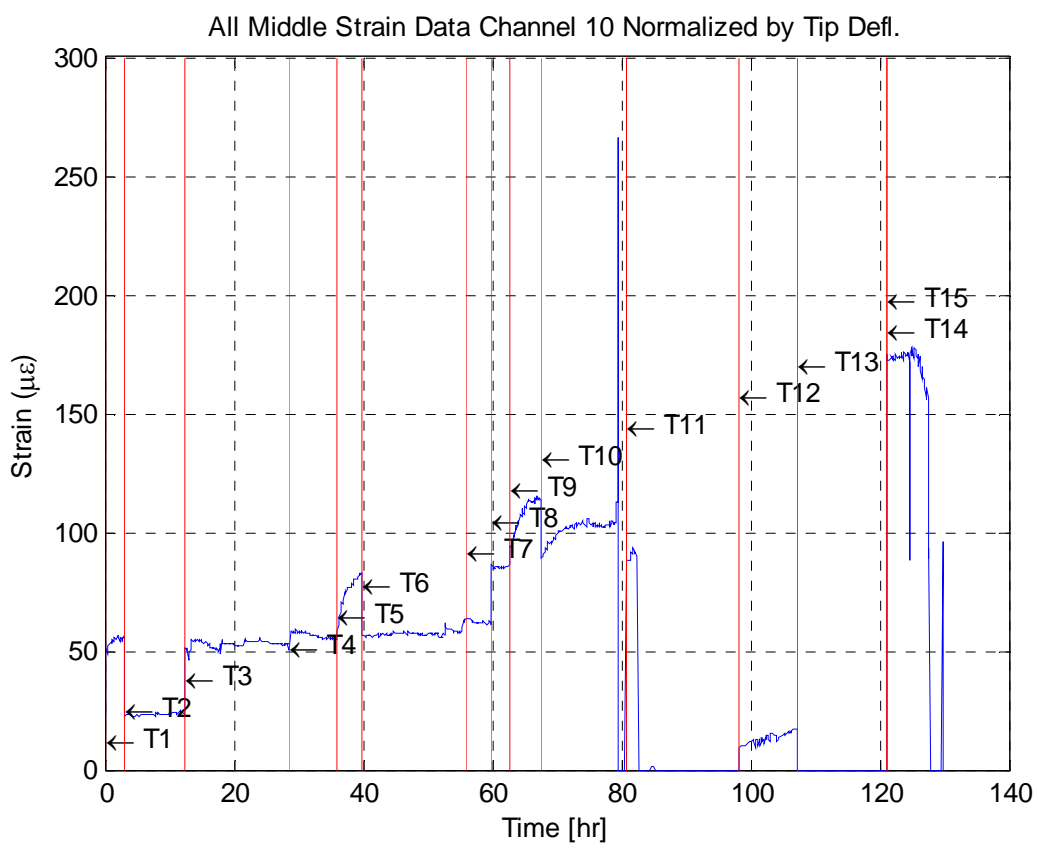

Figure 6-37. All trailing edge strain gage data for full scale fatigue test performed on blade B. Each test is shown with a red vertical line and a T\# indicator. 


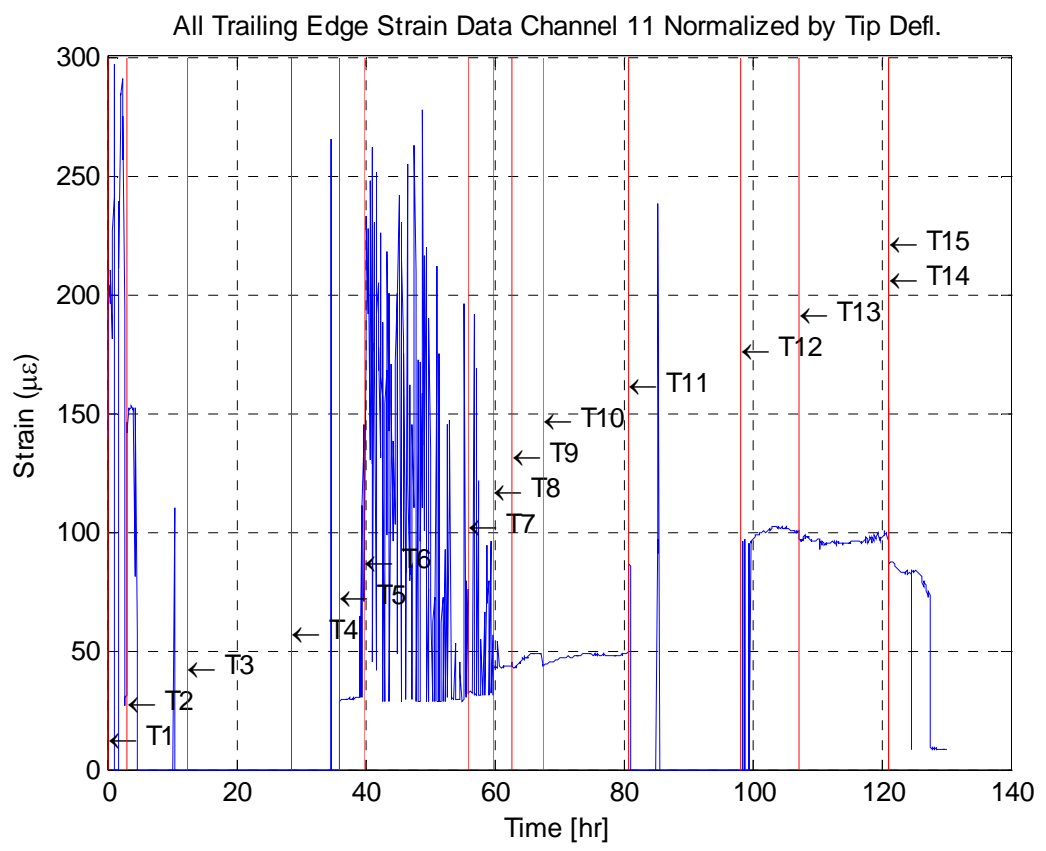

Figure 6-38. All trailing edge strain gage data for full scale fatigue test performed on blade B. Each test is shown with a red vertical line and a T\# indicator.

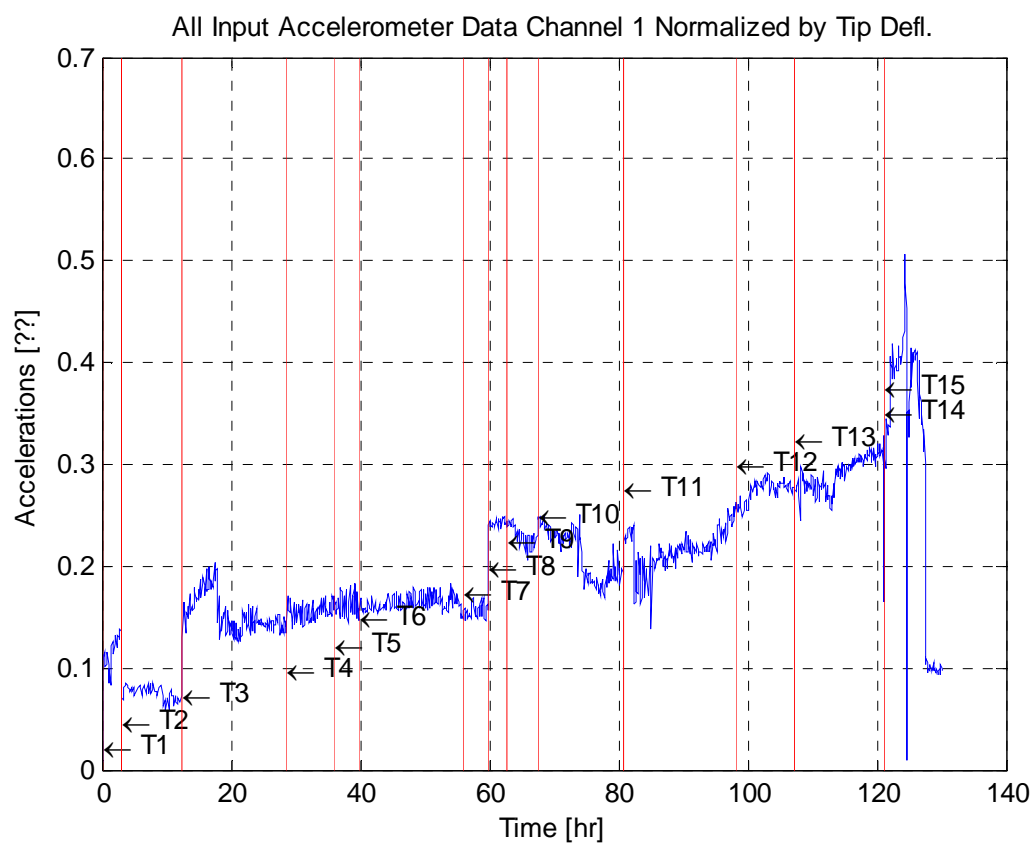

Figure 6-39. All input accelerometer data for full scale fatigue test performed on blade B. Each test is shown with a red vertical line and a T\# indicator. 


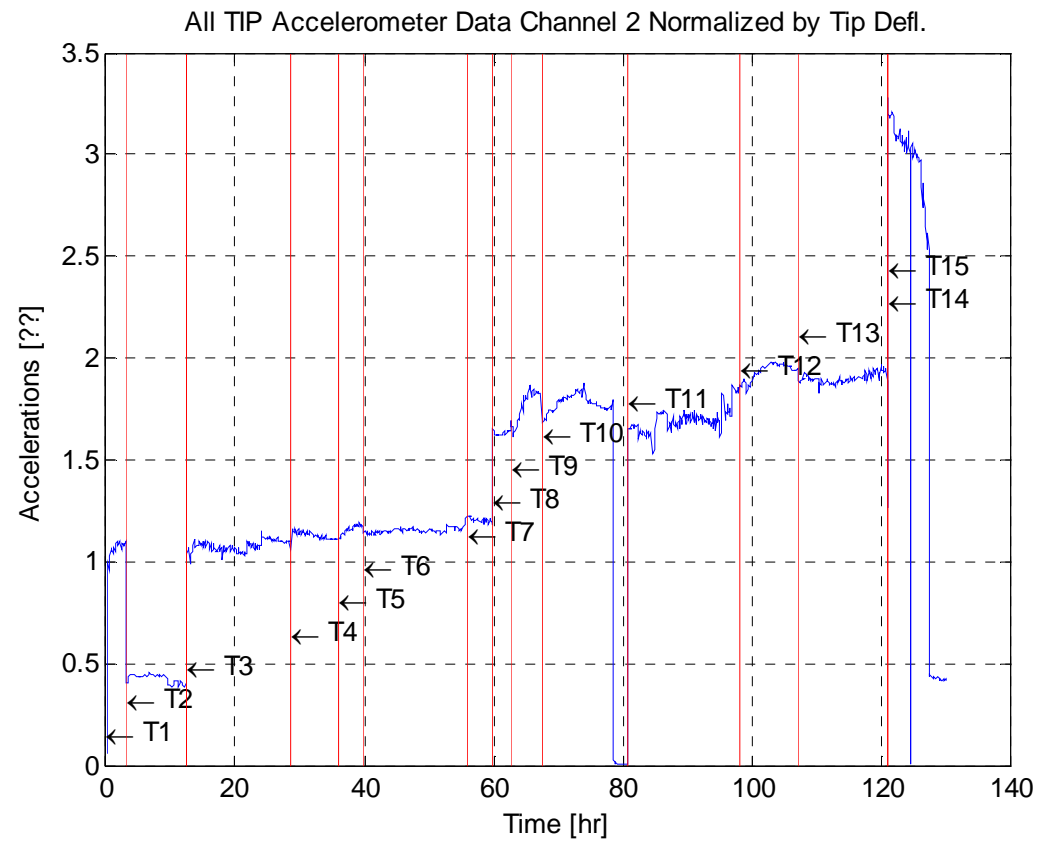

Figure 6-40. All blade tip accelerometer data for full scale fatigue test performed on blade B. Each test is shown with a red vertical line and a T\# indicator.

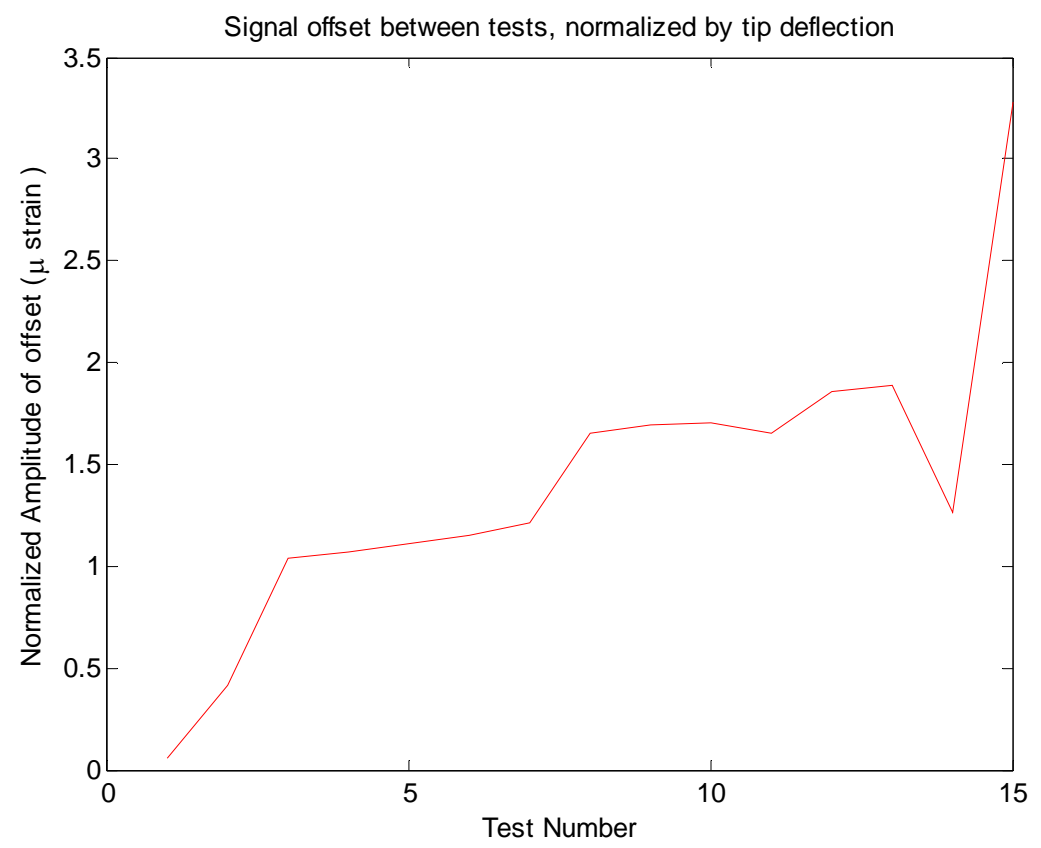

Figure 6-41. Signal offset between consecutive tests normalized by blade tip deflection for blade B. 
finalmat ( (2:end), 1)=labelrow;

finalmat $(1,(2$ : end $))=1$ abelcol;

finalmat $(2$ : end, 2 : end $)=r f m$;

\section{Rainflow Cycle Count Histogram Function}

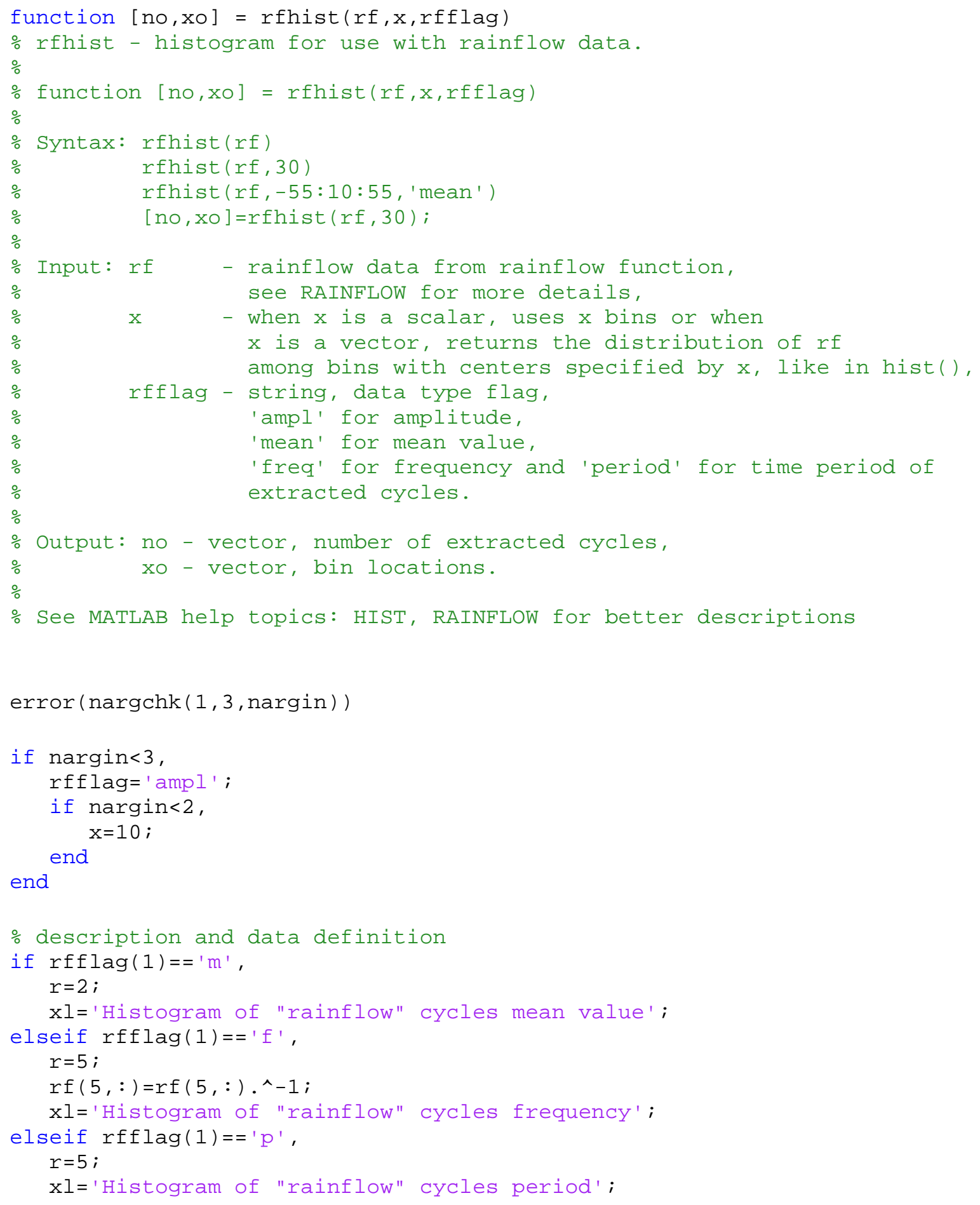




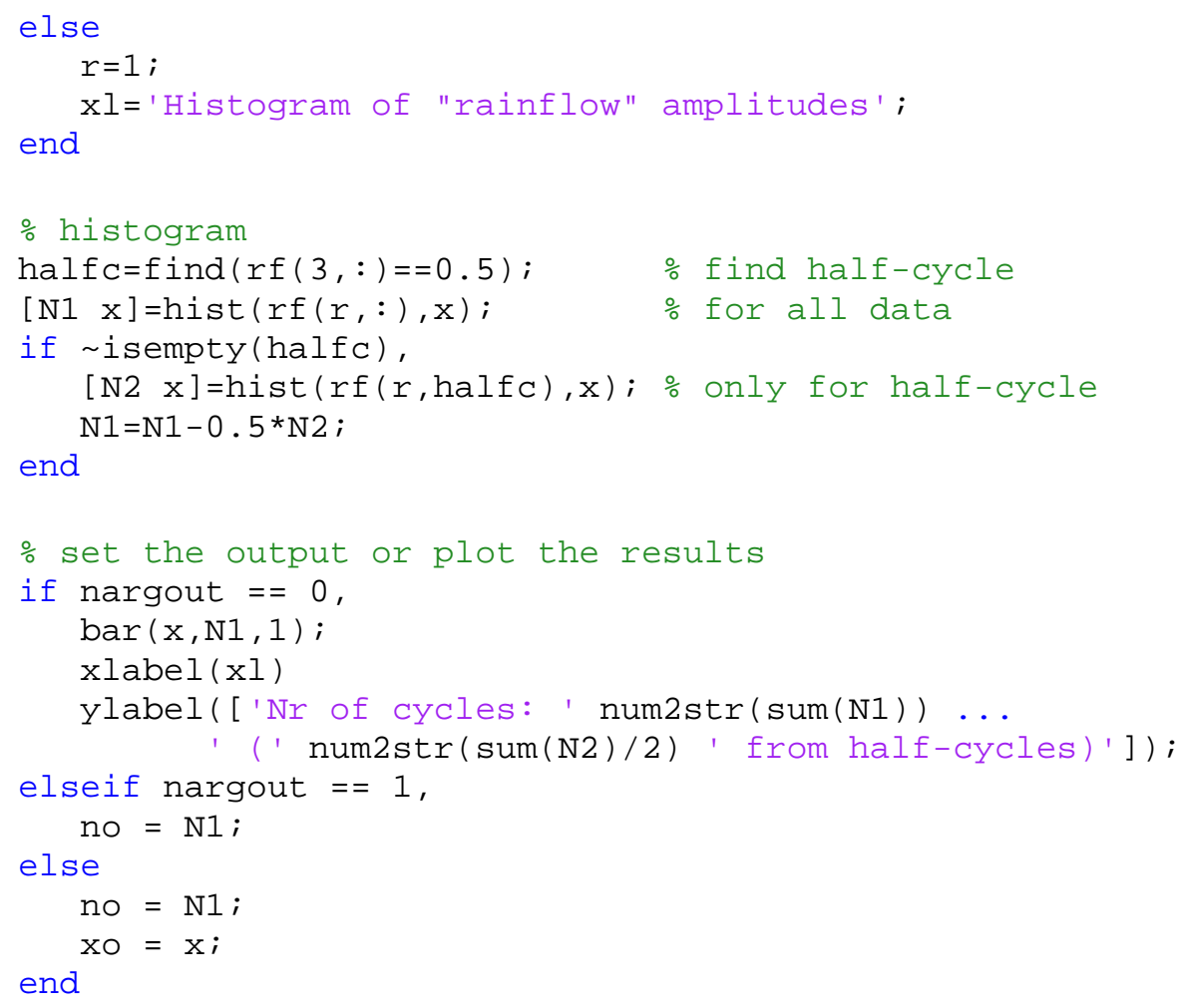

\section{Rainflow Cycle Count Matrix Function}

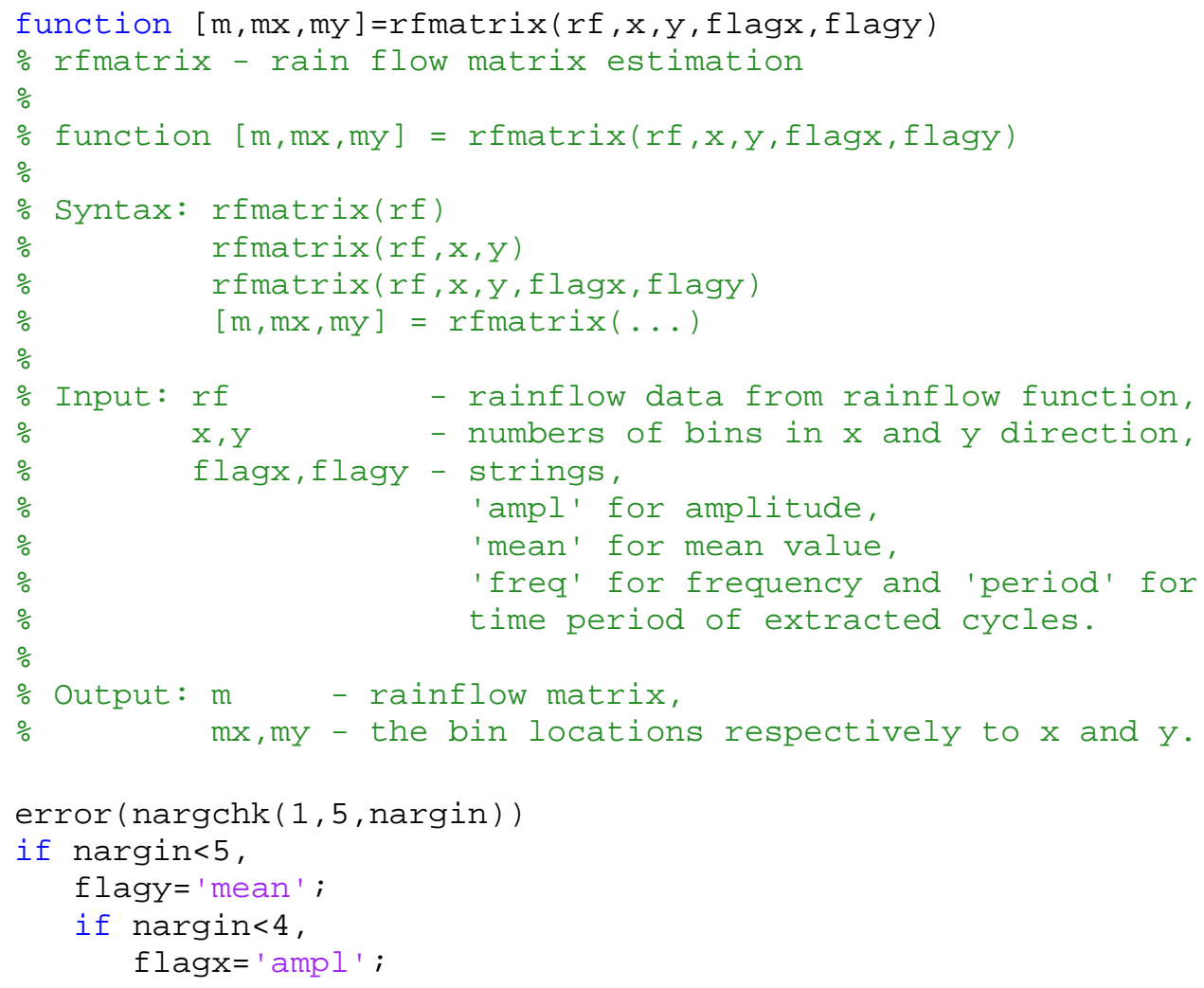




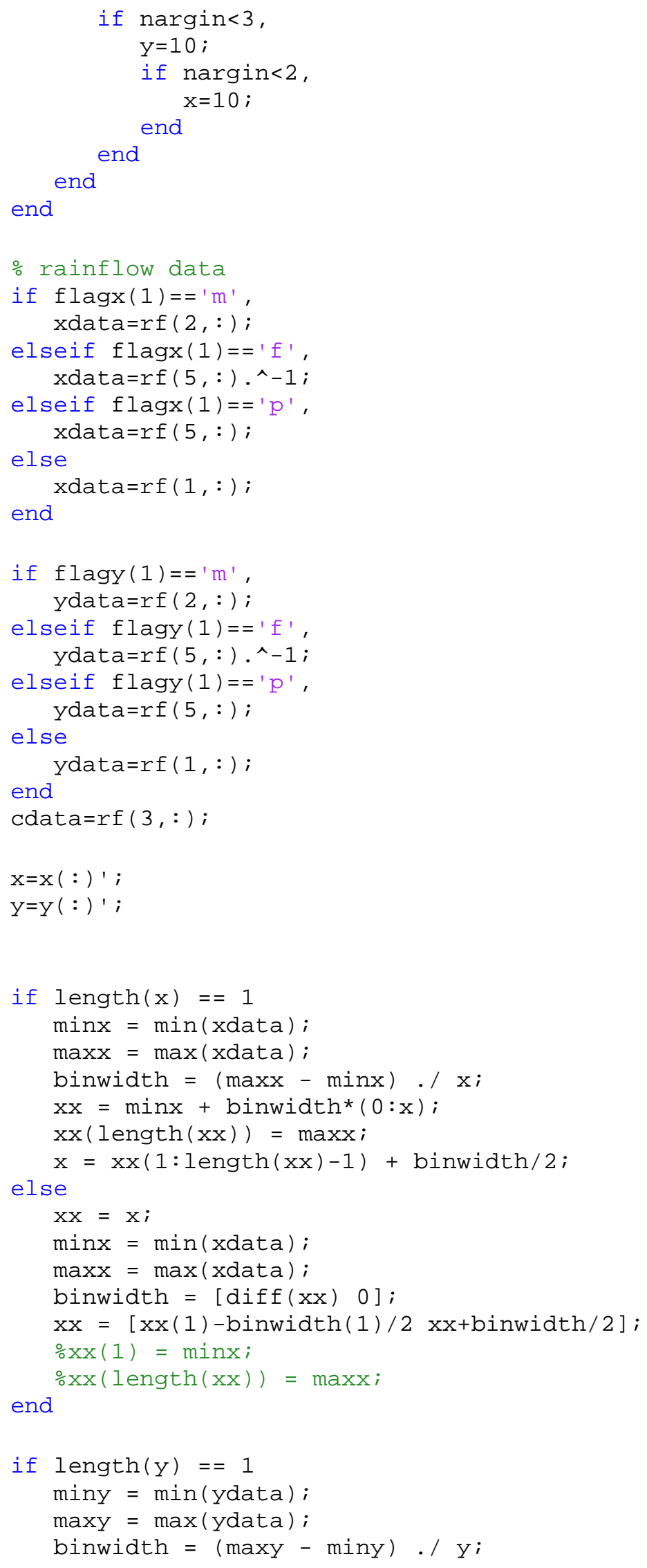




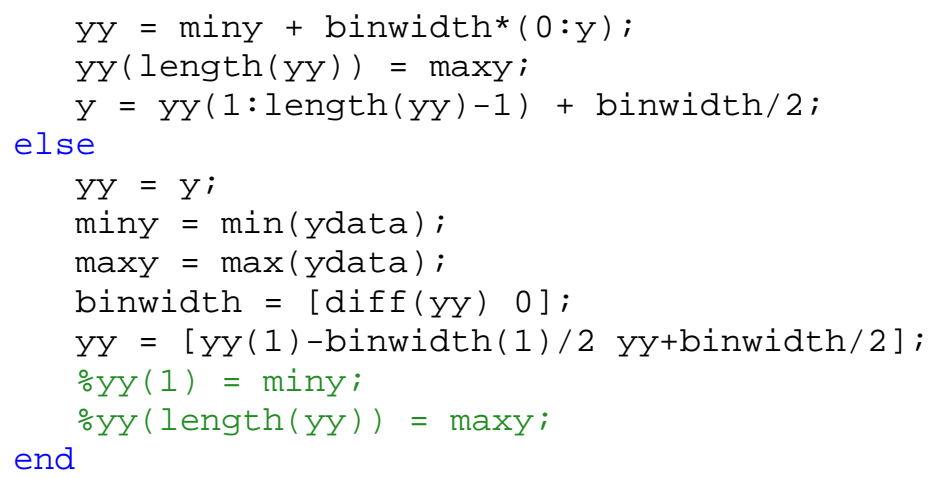




\section{Numerical Blade Response Model}

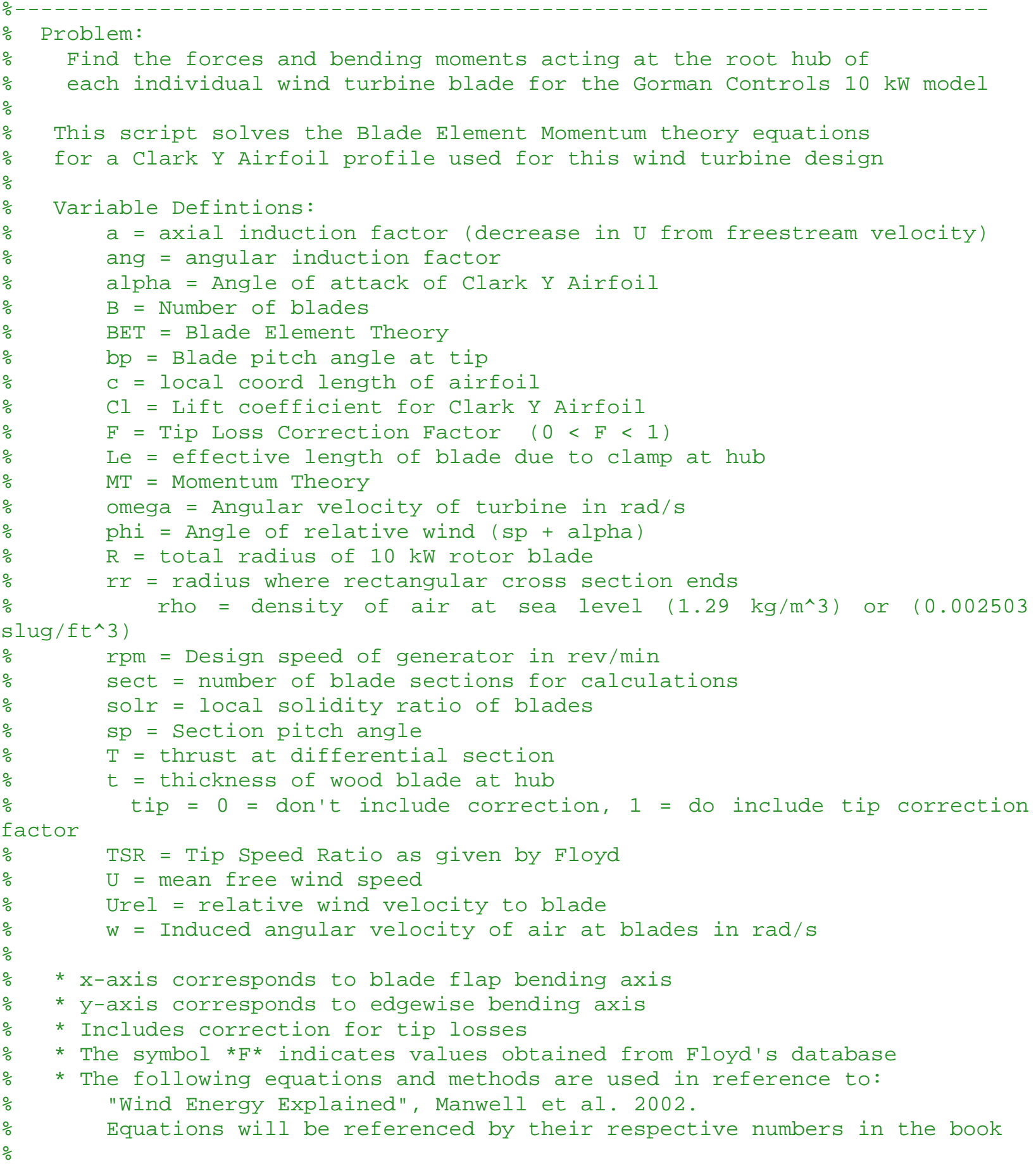




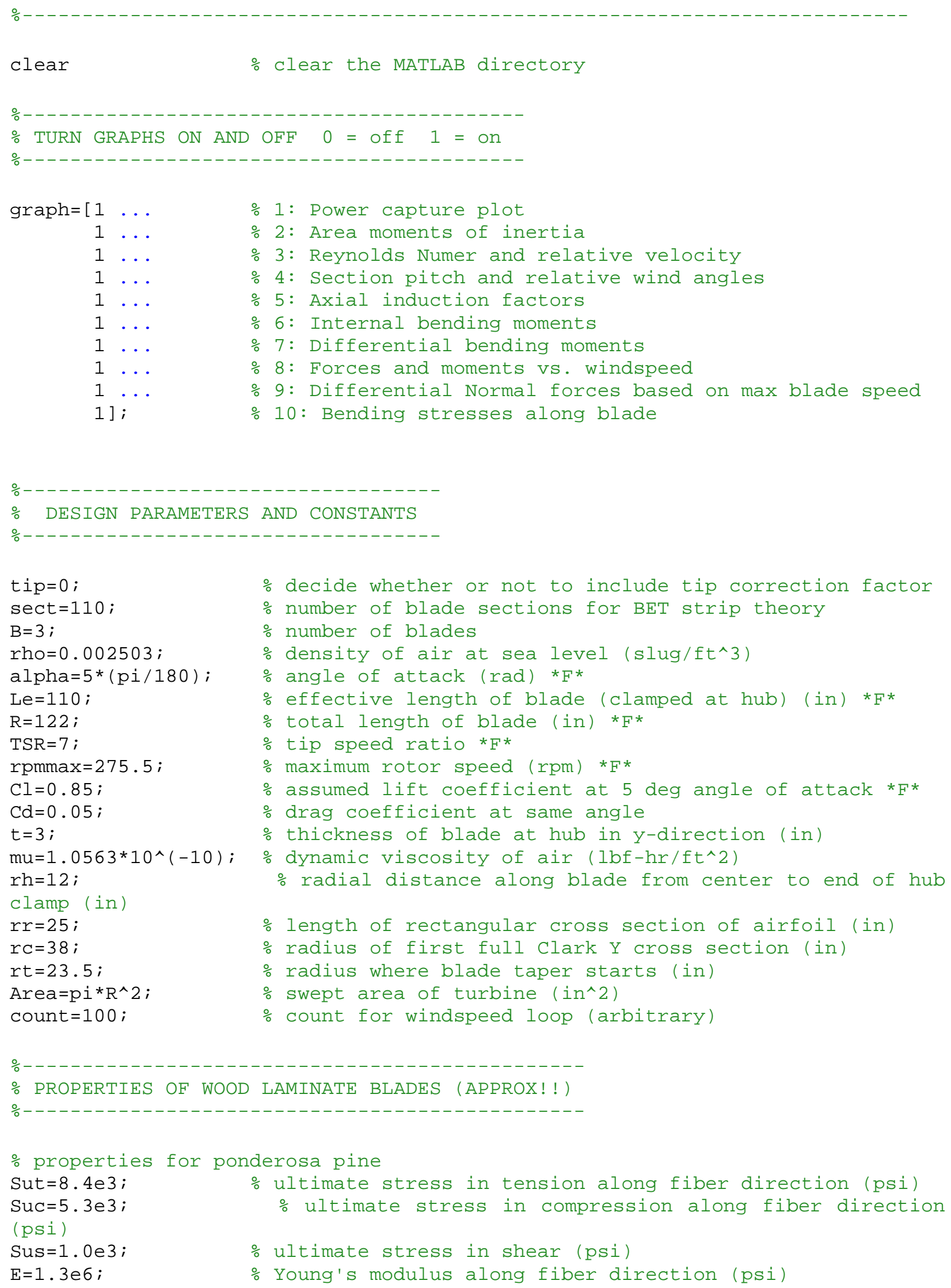




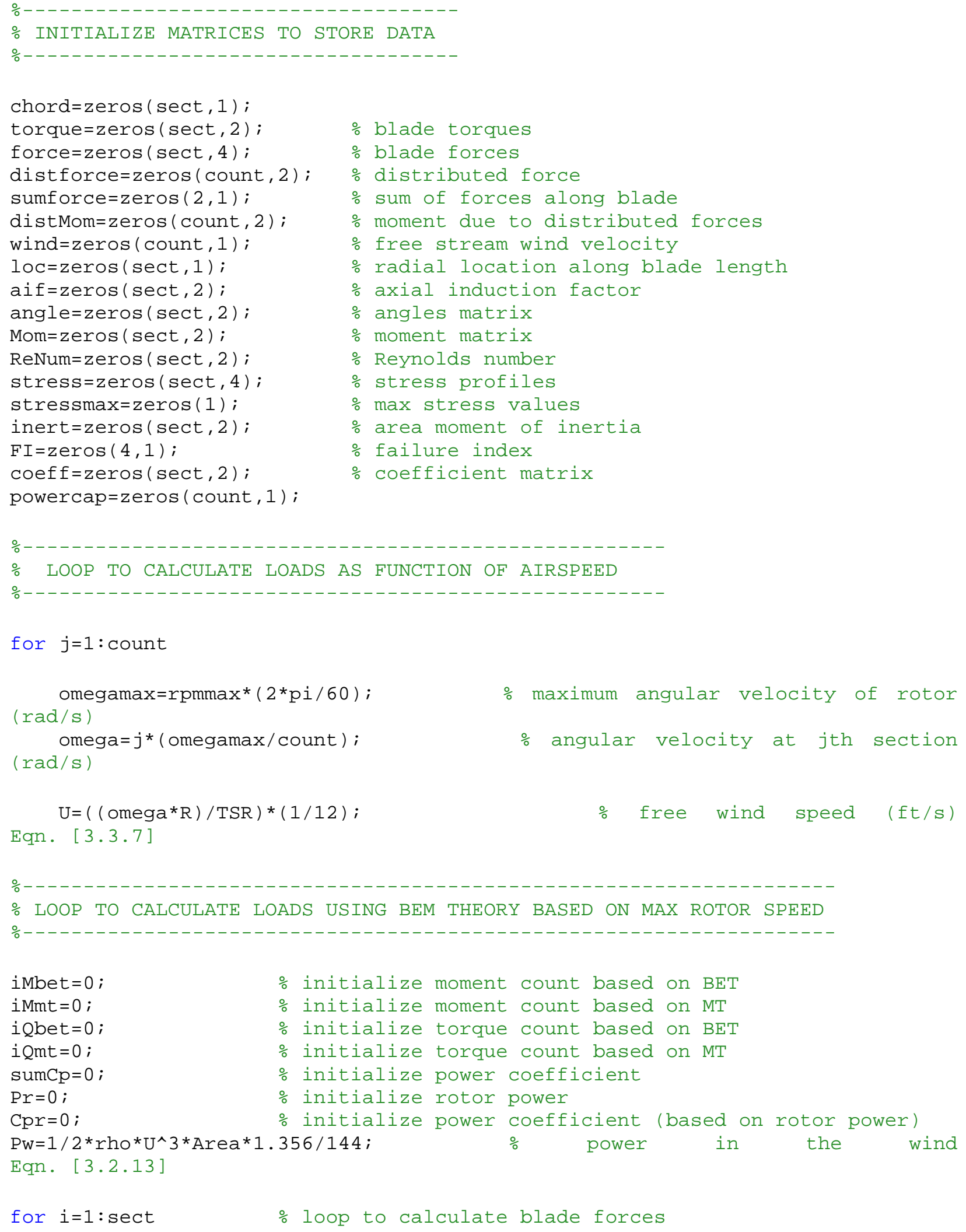




$$
\text { (in) }
$$$$
d r=\text { Le / sect ; }
$$$$
r=r h+(d r * i)-d r / 2 \text {; }
$$

Eqn. [3.3.8]
\% annular ring airfoil section thickness \% current radial location (middle of \% local tip speed ratio

\% Select whether in Clark Y section or rectangular section of blade if $r>r r$; $\mathrm{sp}=\operatorname{atan}((2 * \mathrm{R}) /(3 *(\mathrm{r}) * \mathrm{TSR}))-\mathrm{alpha} ; \quad \frac{\circ}{0}$ current section pitch angle (rad) ${ }^{*} \mathrm{~F}^{*}$ phi $=(\mathrm{sp}+\mathrm{alpha})$;

\% current angle of

relative wind (rad)

elseif $r<=r r$; phi=atan (2/(3*LTSR)); $\quad$ units (rad)

Eqn. [3.8.3] alphafp=phi; $\quad$ for flat plate only

end $\mathrm{sp}=0$;

\% zero twist angle

$[c]=\operatorname{chordprop}(r, r t, r r, s p) ; \quad$ o compute the local chord length for ith element (in) $\operatorname{sol} r=(B * C) /(2 * p i * r) ; \quad$ local solidity ratio Eqn. $[3.7 .3]$

$\circ$ Clift $=4 * \sin (p h i) *\left[\left[\cos (p h i)-L T S{ }^{*} \sin (p h i)\right] \ldots \%\right.$ calculate lift coeff. $\left.\% /\left[\operatorname{sol}{ }^{*}\left(\sin (\mathrm{phi})+\operatorname{LTSR}{ }^{*} \cos (\mathrm{phi})\right)\right]\right] ;$ $\mathrm{a}=1 /\left(1+\left(4{ }^{\star} \sin (\mathrm{phi}) \wedge 2\right) /\left(\operatorname{sol}{ }^{\star} \mathrm{Cl}{ }^{*} \cos (\mathrm{phi})\right)\right) ; \quad \%$ axial induction factor Eqn. $[3.7 .9]$

잉 $\mathrm{ang}]=$ afactors $($ LTSR, phi, sp, r, t, B, c, solr $)$

if $($ tip $==1) \& \&(r>r r)$; $\quad$ include tip loss coefficient

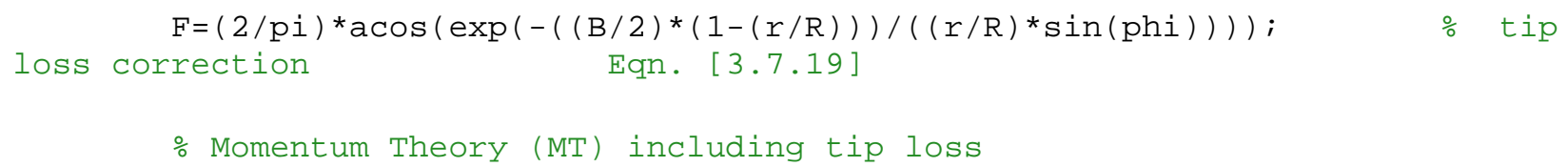




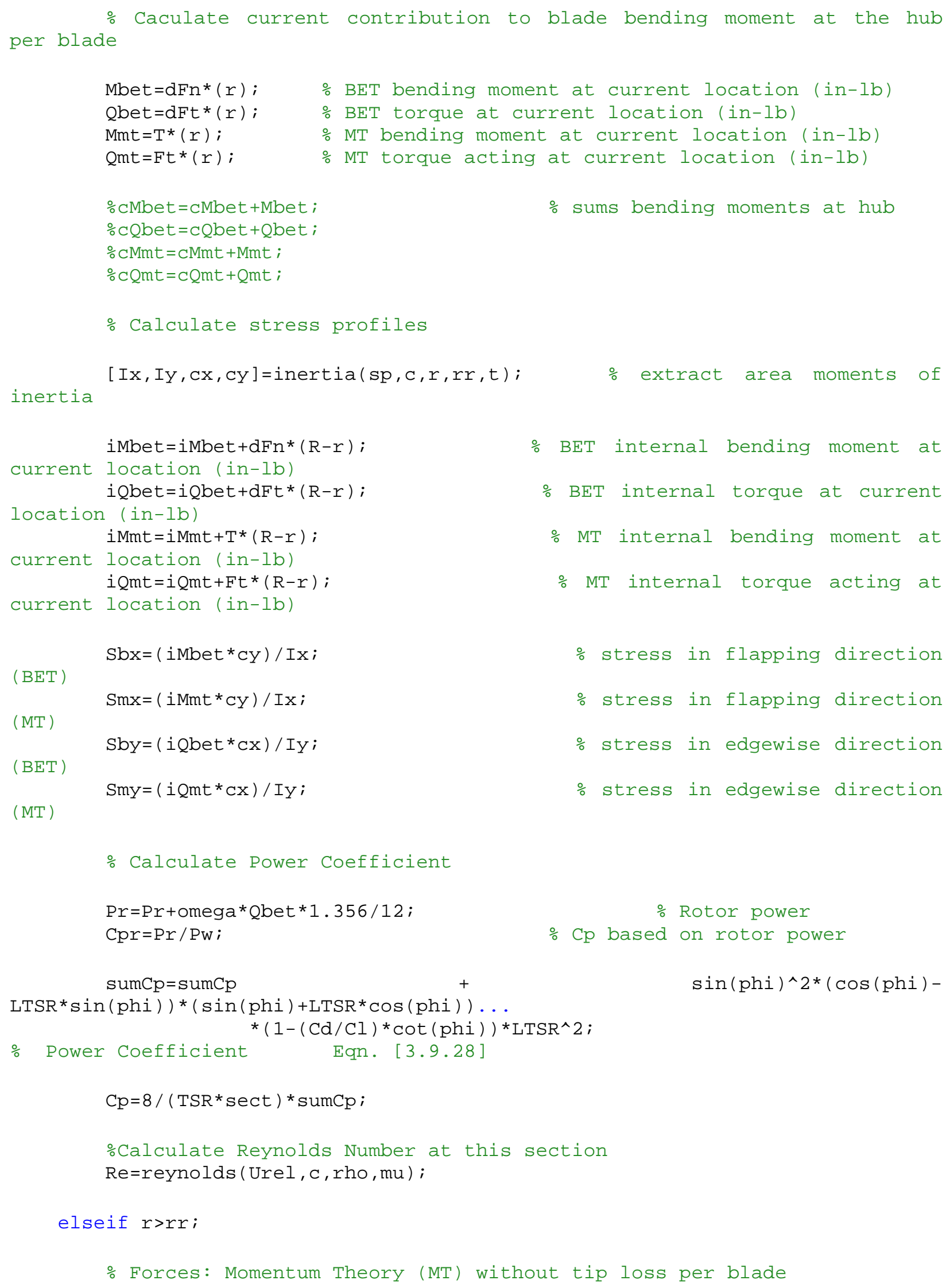




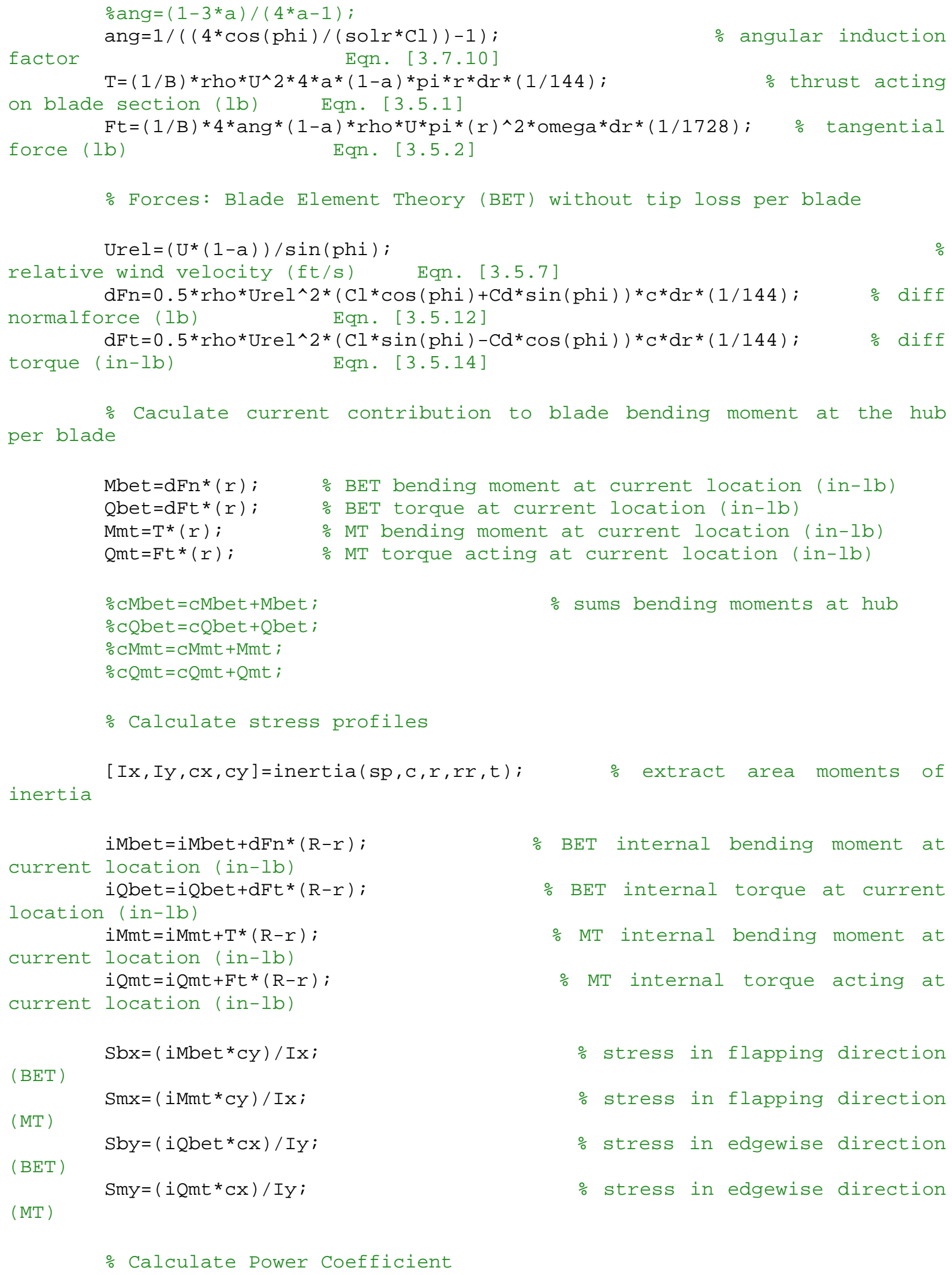




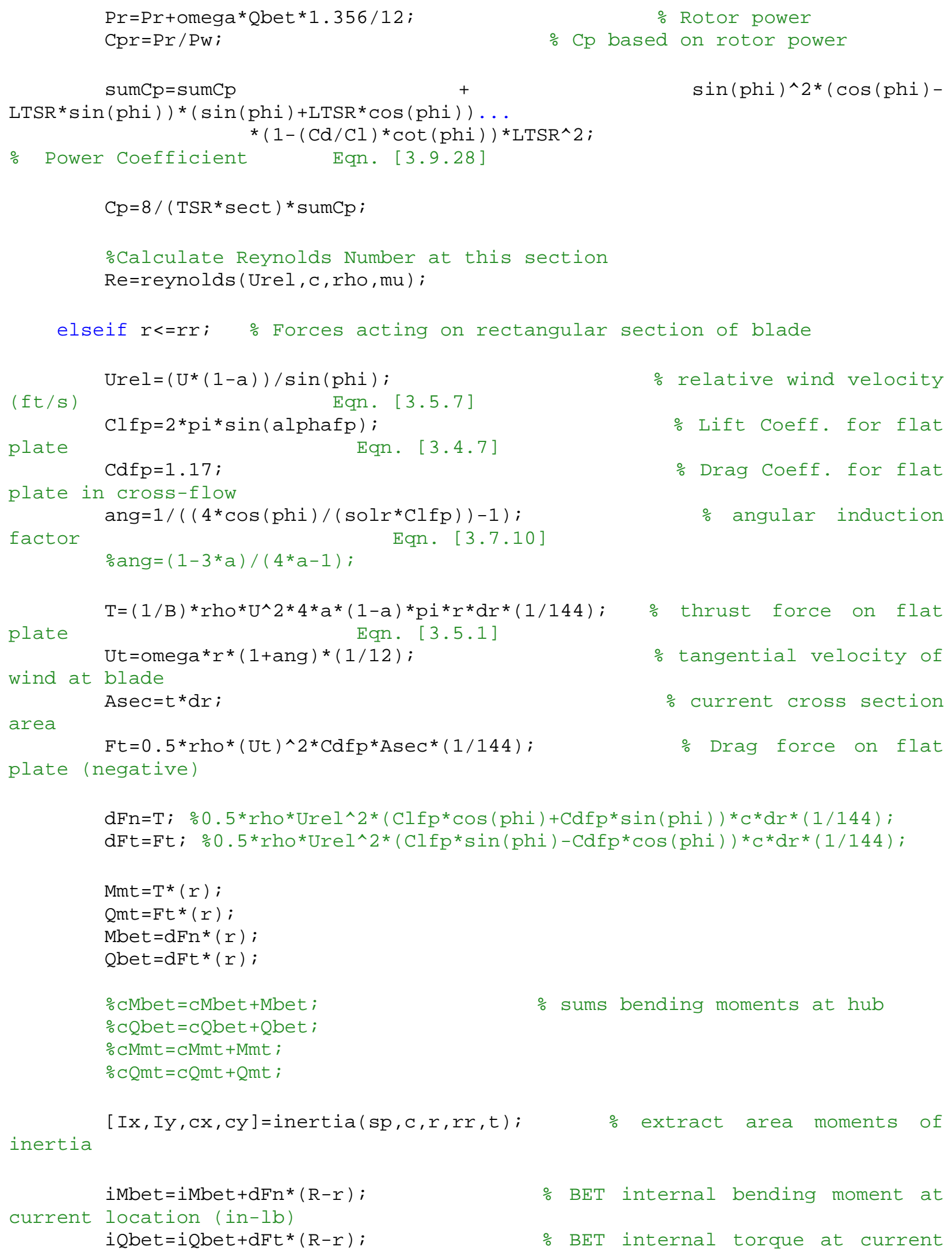




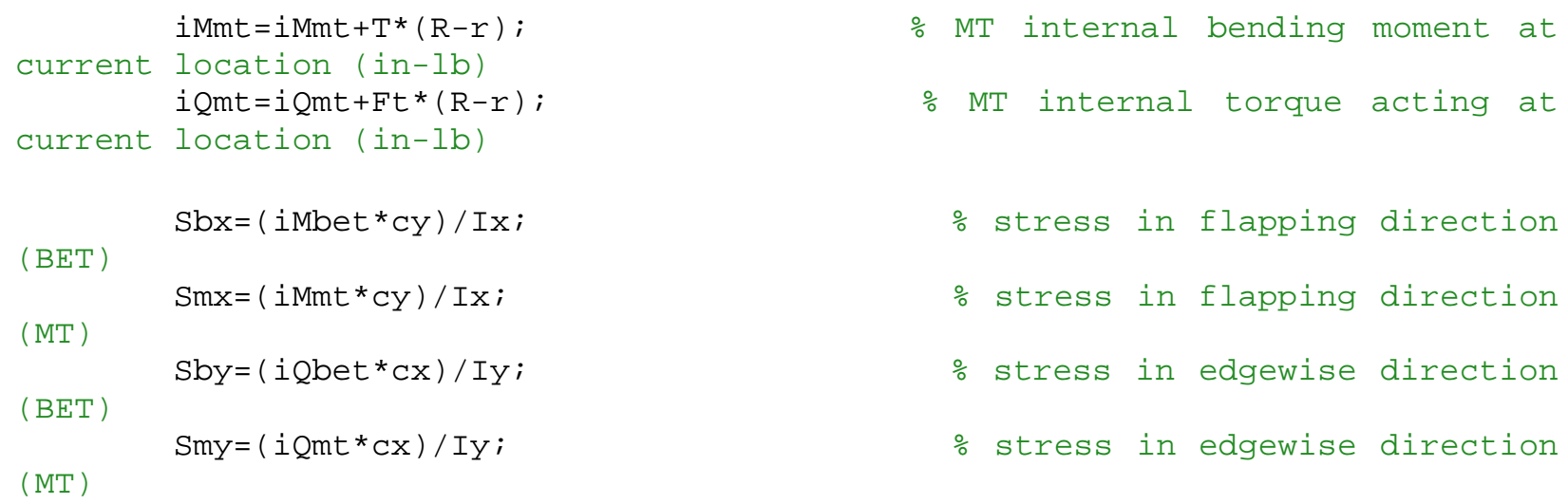

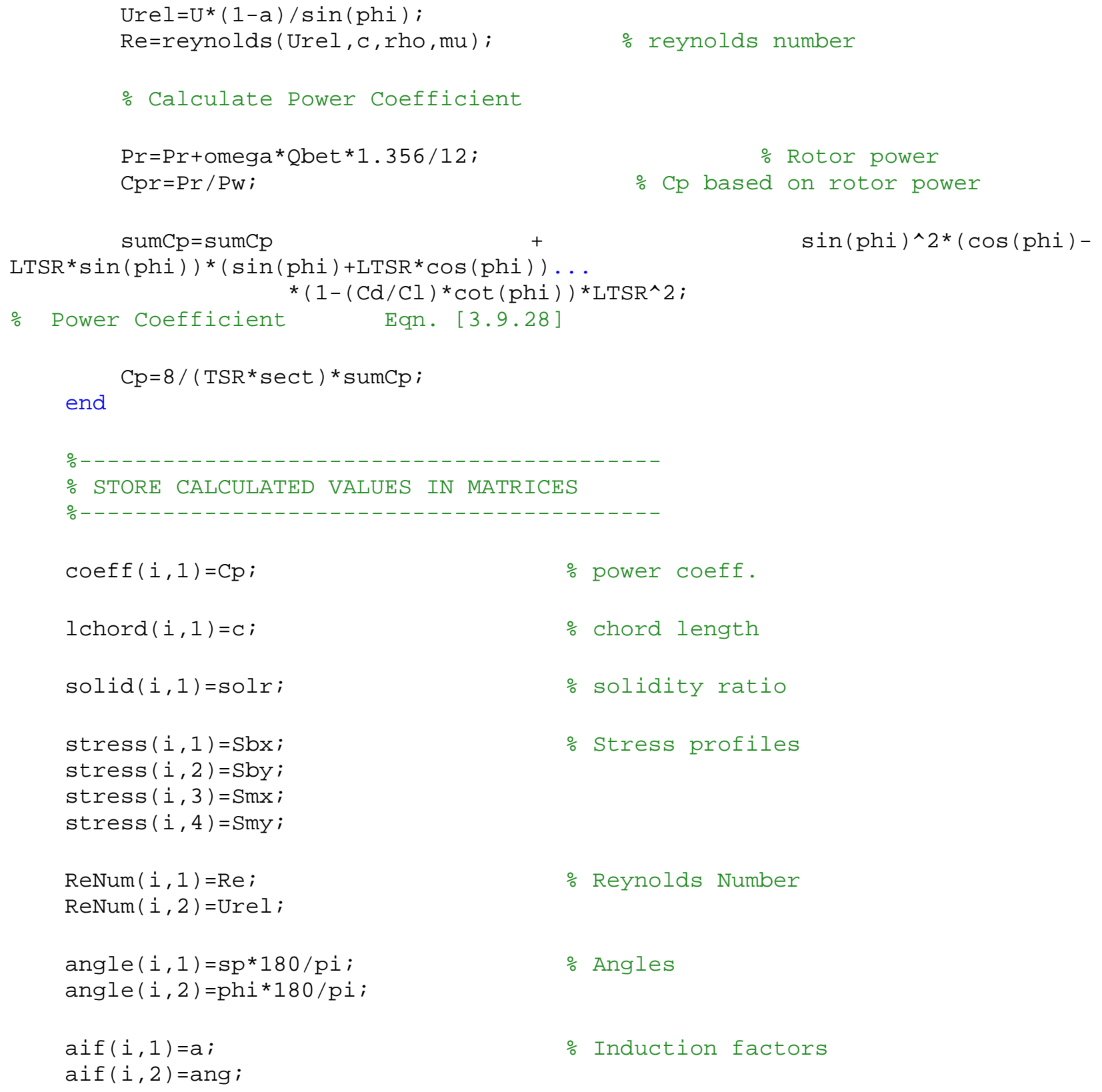




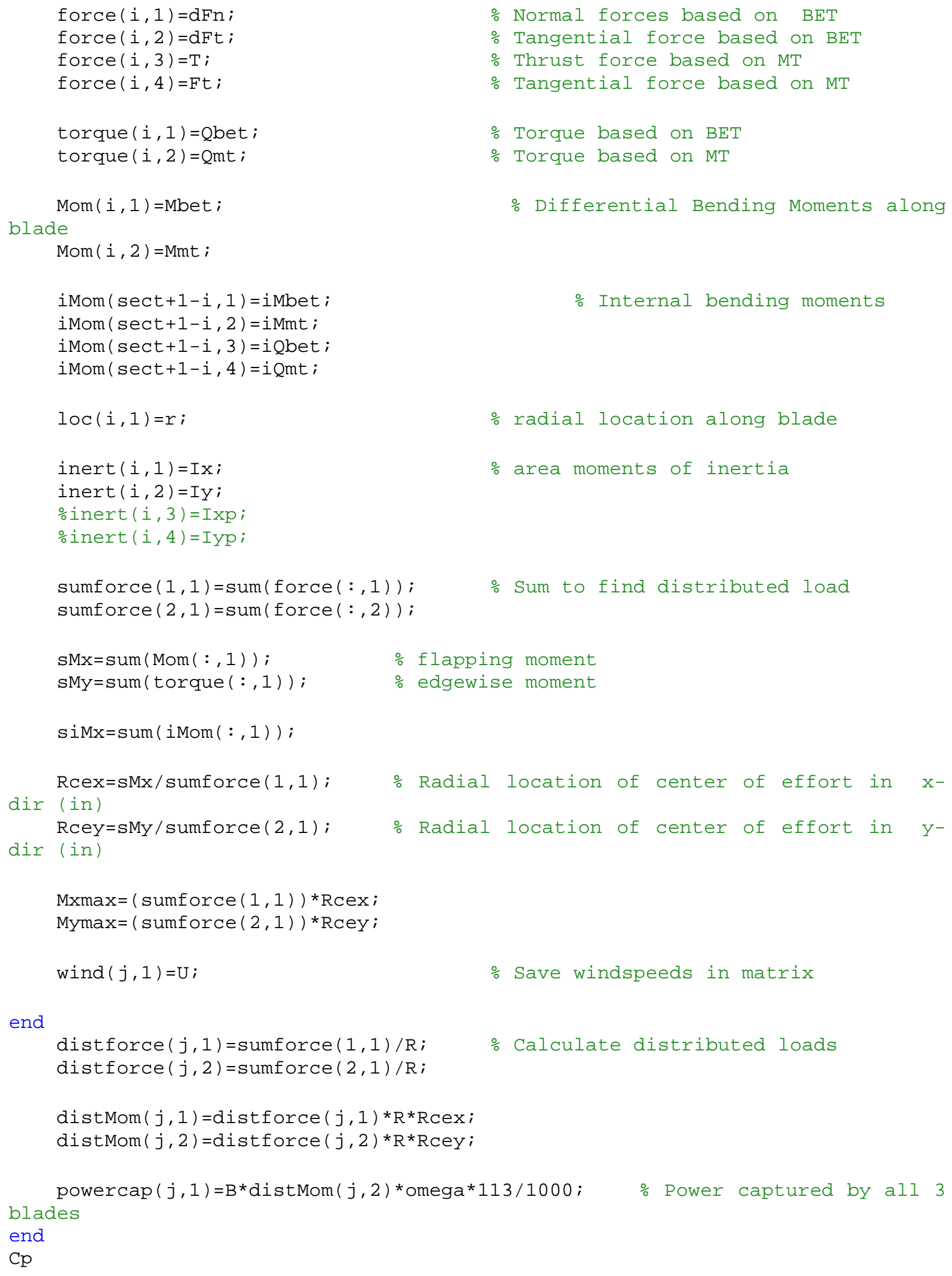




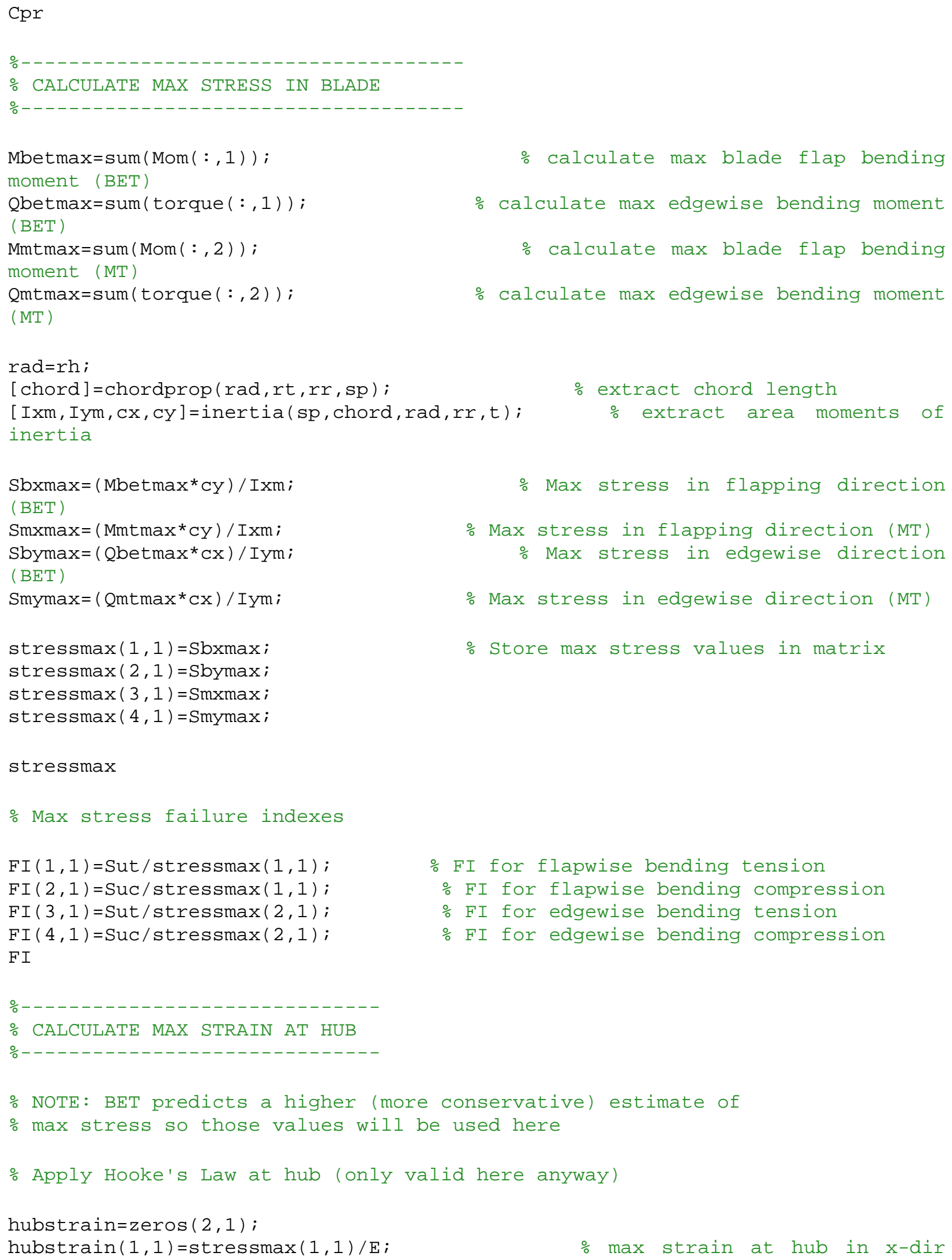




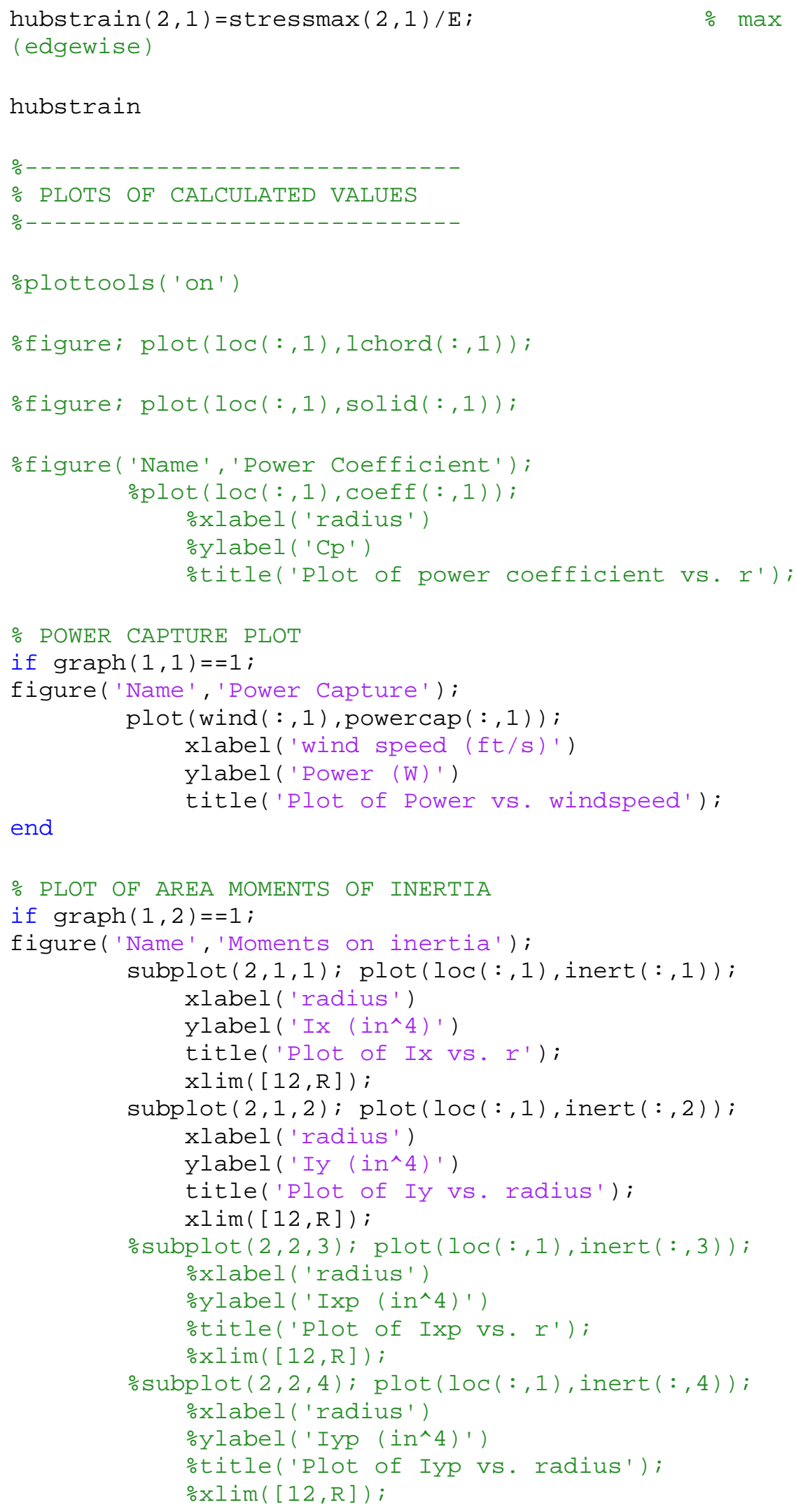

end 


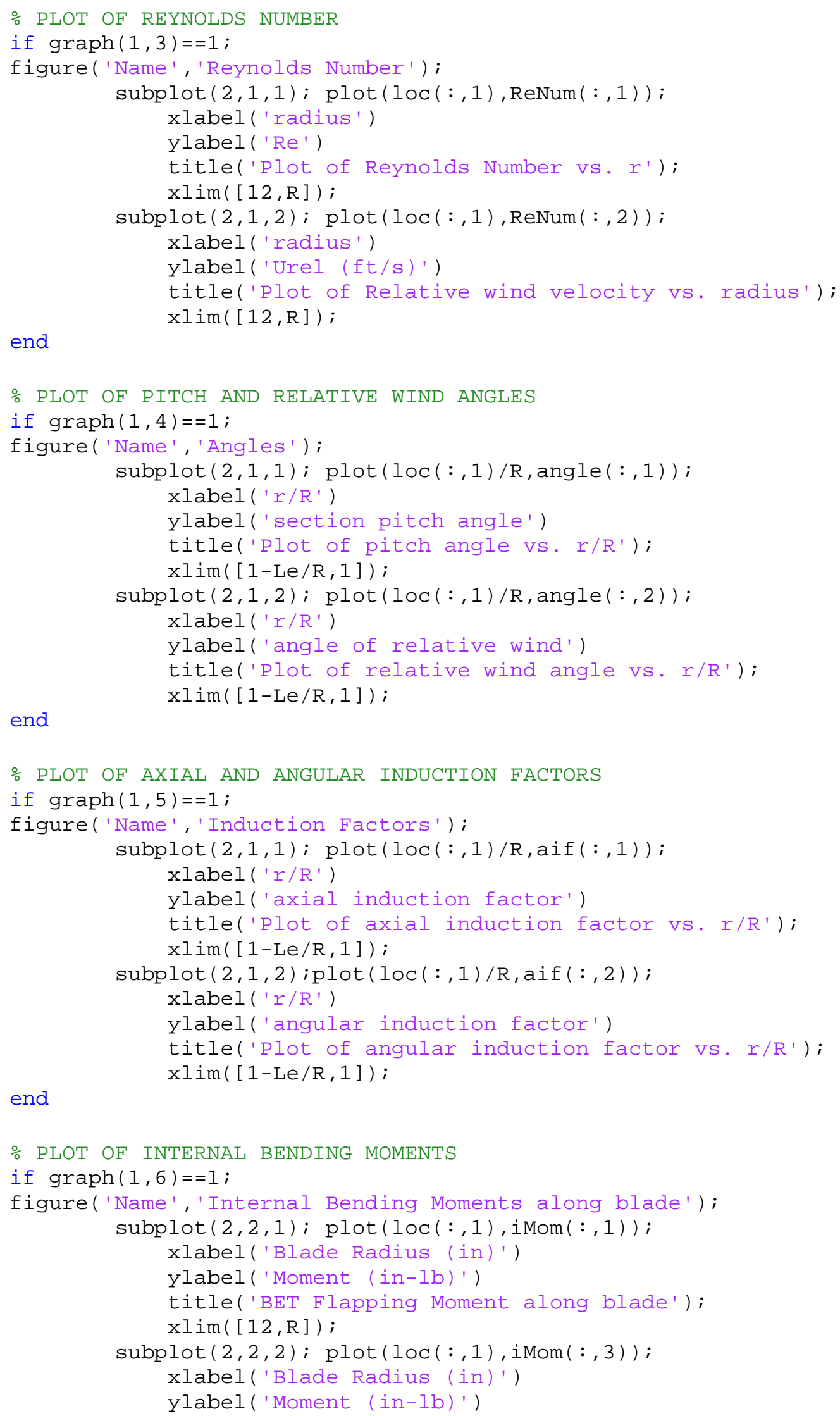




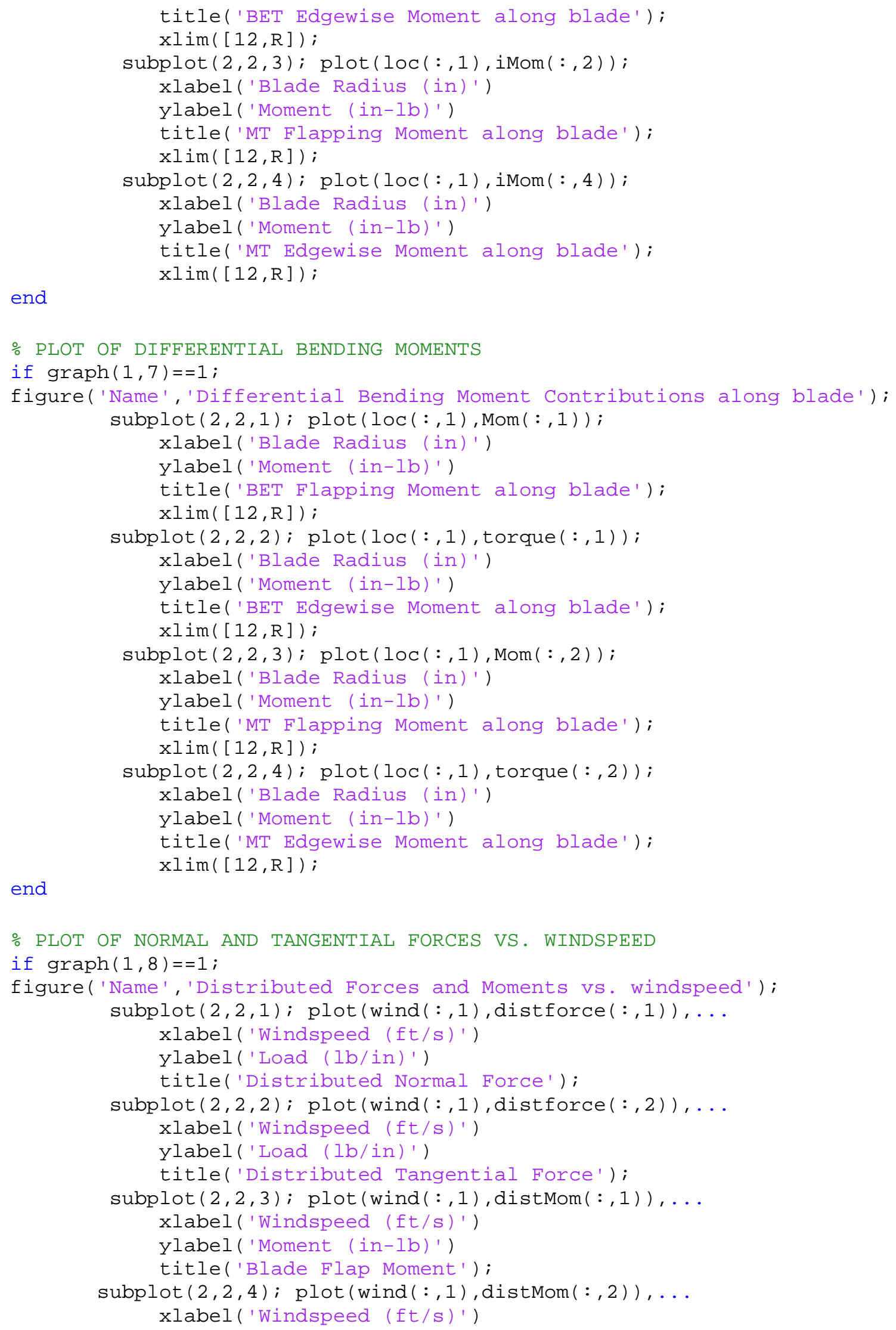




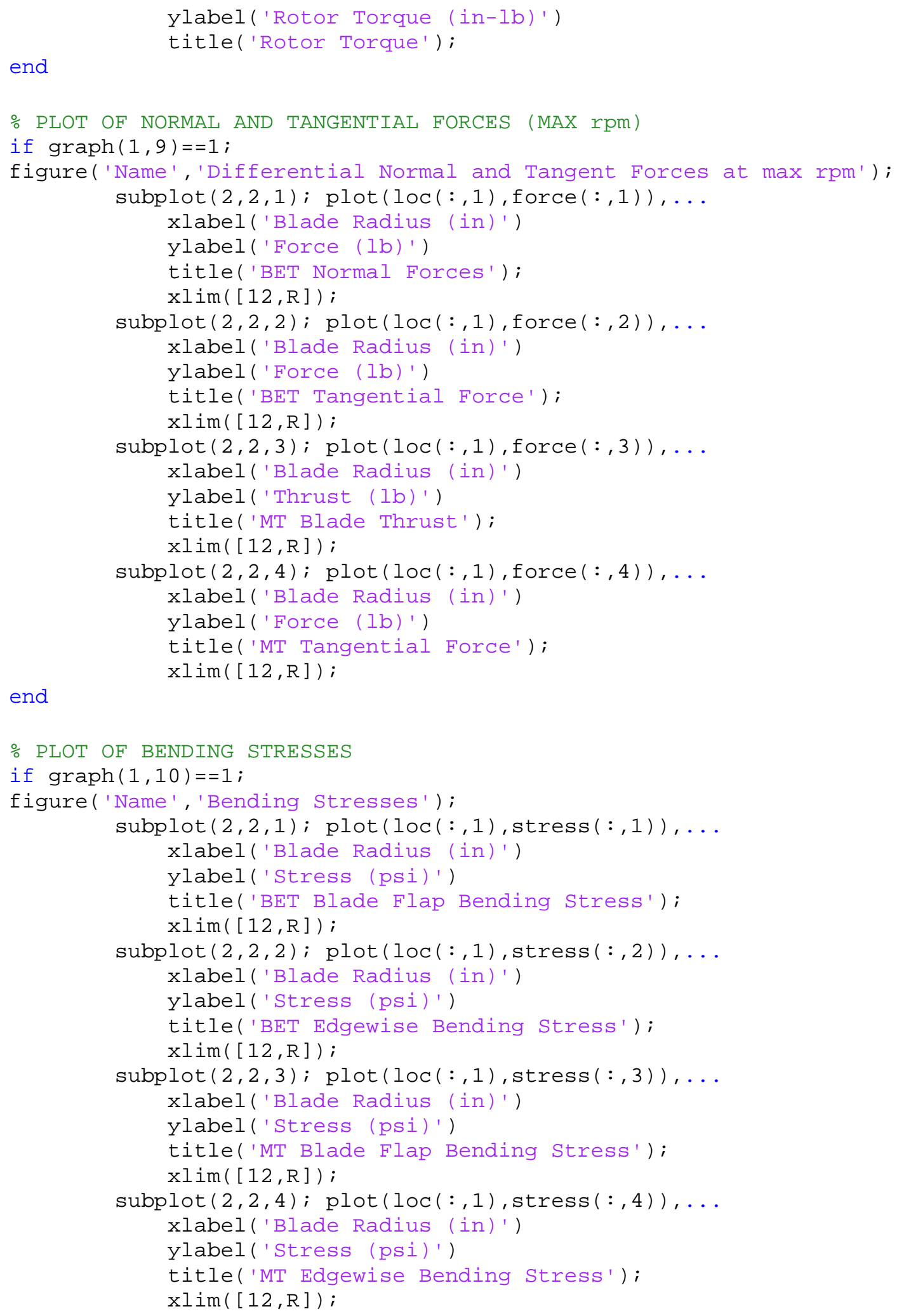

end

\section{Mykelstad Method/Rotating Beam Natural Frequency Calculator}




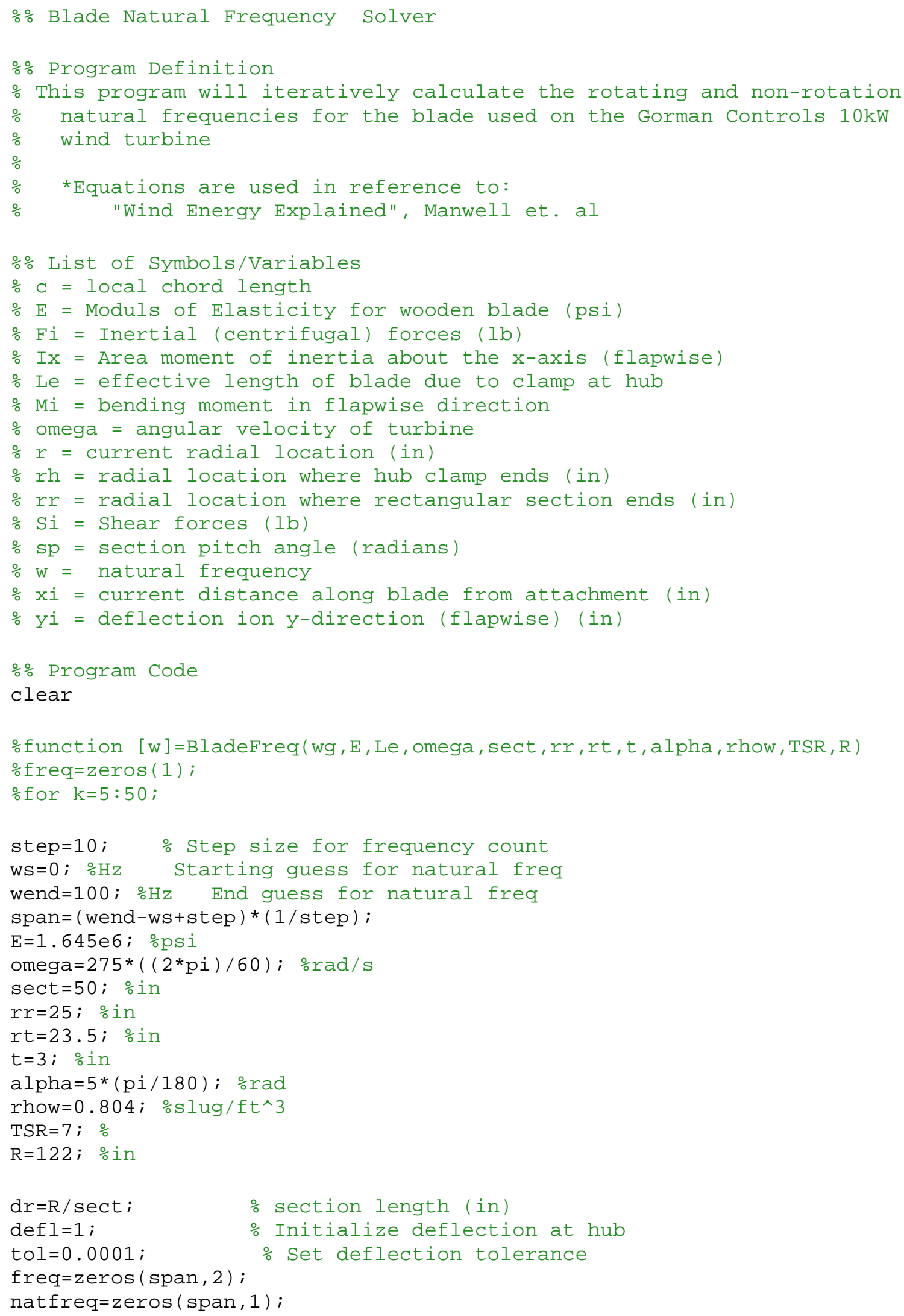


end

\% Calculation \#2: Starting at free end of blade

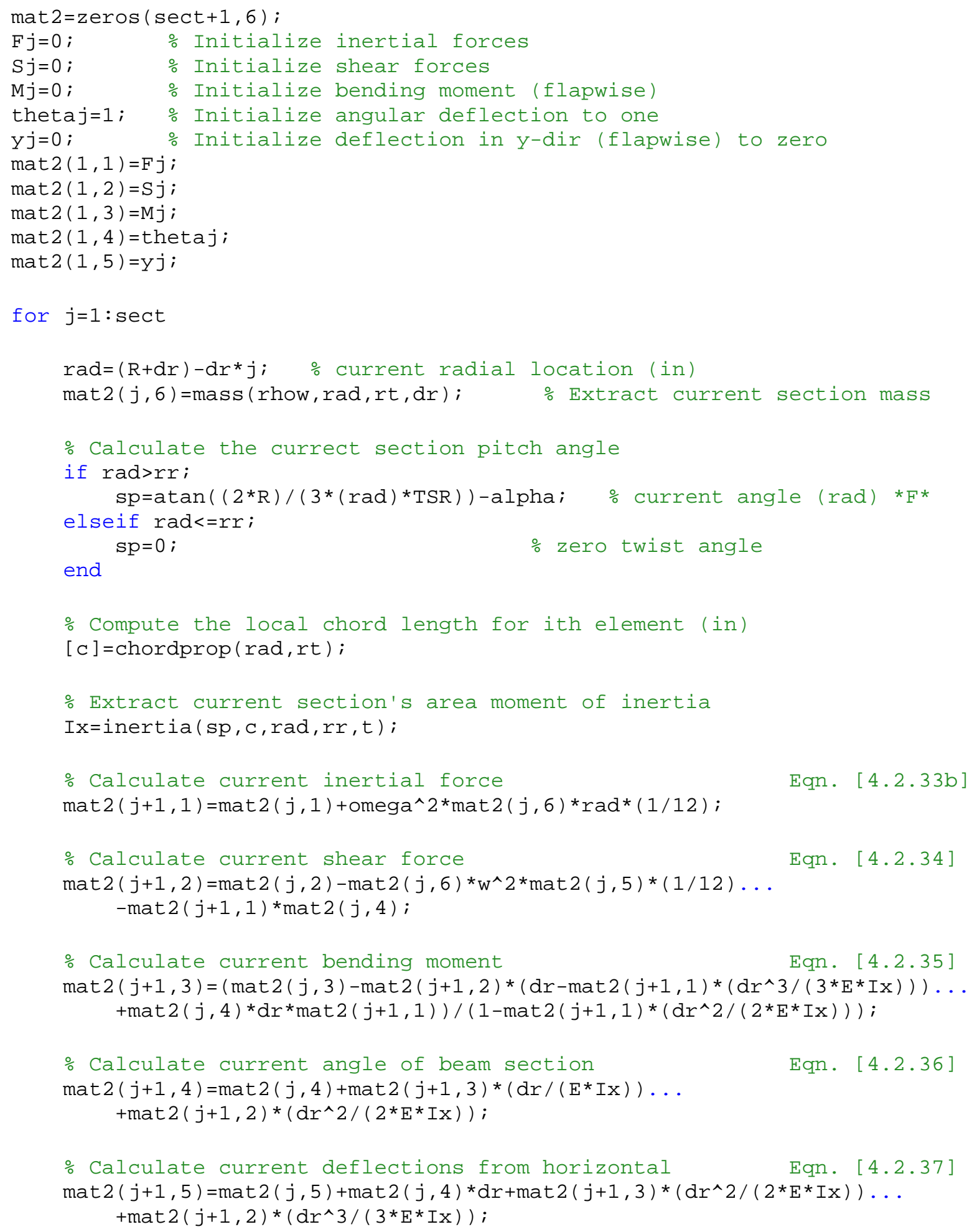




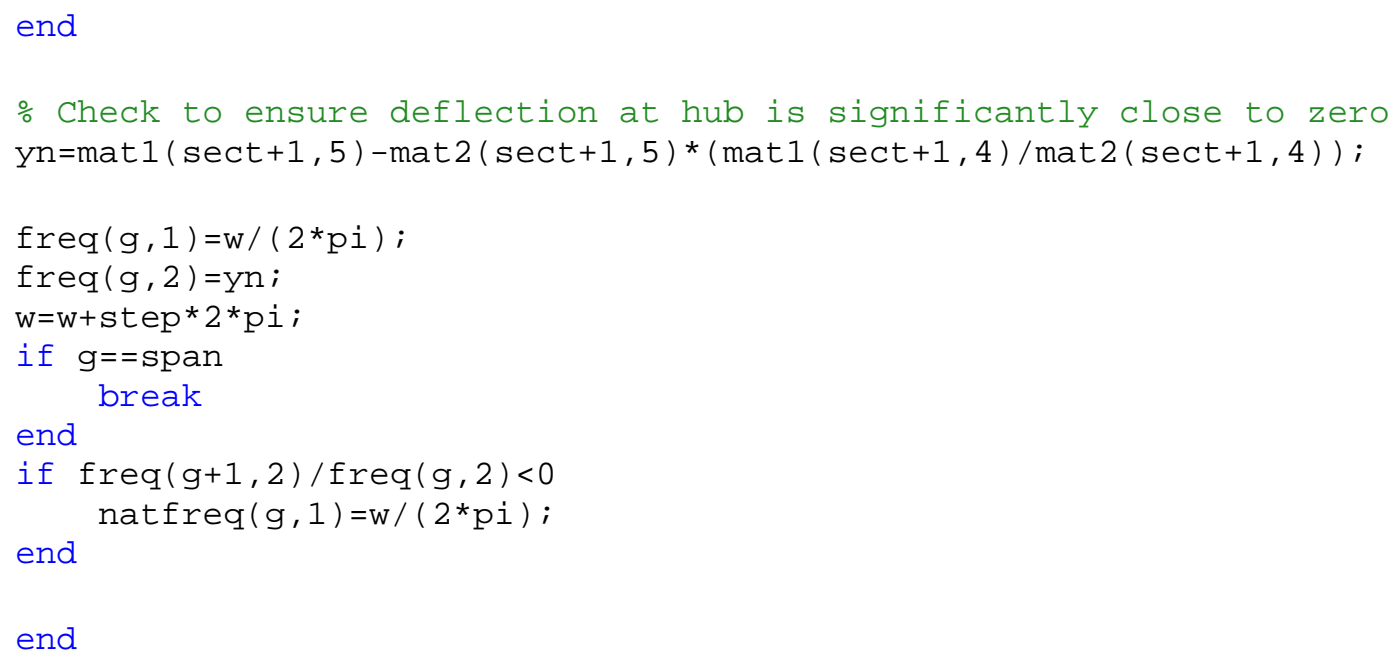

\section{Data Acquisition MATLAB Script}

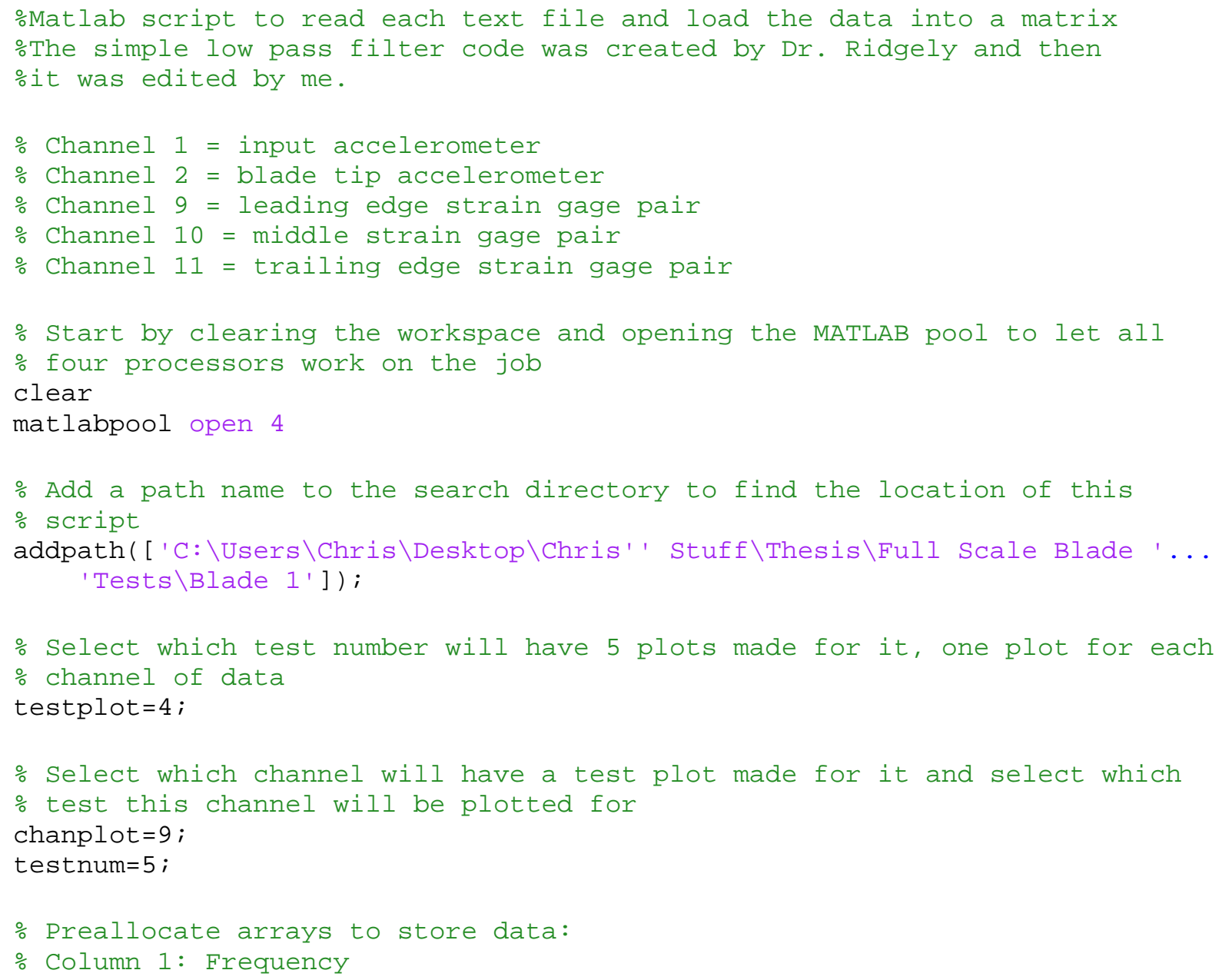


\% Column 2: Amplitude

- Column 3: Time

allaccl=zeros $(0,3)$;

allacc $2=\operatorname{zeros}(0,3)$;

allstr $9=$ zeros $(0,3)$;

allstr $10=$ zeros $(0,3)$;

allstr $11=\operatorname{zeros}(0,3)$;

ntest $=10 ; \%$ There are 6 tests so far

nchan $=5$; $\frac{\circ}{\circ}$ Number of data channels

- Create an array to store the time categories for each test along with the

\% length of each individual test and the deflection at each test

timecat $=$ zeros (ntest, 3$)$;

\% Normalize data by tip deflection during test

timecat $(:, 3)=[17,17,17,17,17,17,16.5,16.75,16.5,16]$ ';

\% Create matrix to store time stamps and before/after data

timestamps=zeros (ntest, 3$)$;

bfaf=zeros $(1,2)$;

\% Assign the current directory to locate the files for each test

ㅇ the $\mathrm{k}$ value indicates which test number to search for

for $\mathrm{k}=1$ : ntest

testID=int $2 \operatorname{str}(\mathrm{k})$;

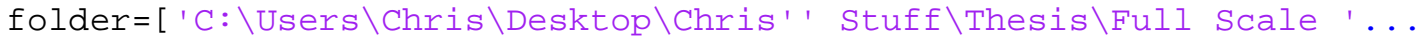
'Blade Tests $\backslash$ Blade $1 \backslash$ Test ' testID];

ochange the current directroy to folder above

cd (folder);

\% Declare a start time for the experiment, this happens to be the first

o data file in the Test 1 folder

if $\mathrm{k}==1$

[strtname]=dir('input1(t) Mar 23, 2009 17-02-44.txt');

tstart=strtname (1).datenum;

end

\% Declare sample interval

tsamp=10; \%sample time in minutes

thr=0;

- Create arrays to store data for each channel

\% the Asterisk * represents a wild card or any character

[tfl] =dir ('inputl (t) * * * *.txt') ;

accell=zeros (length (tfl), 3$)$;

accel2=zeros $($ length $(\mathrm{tfl}), 3)$;

strain $9=$ zeros (length $(t f l), 3)$;

strain10=zeros (length $(\operatorname{tfl}), 3)$;

strain $11=$ zeros (length $(\operatorname{tfl}), 3)$;

ocreate a loop to read multiple text files and store data where

\% each j value corresponds to a channel number 


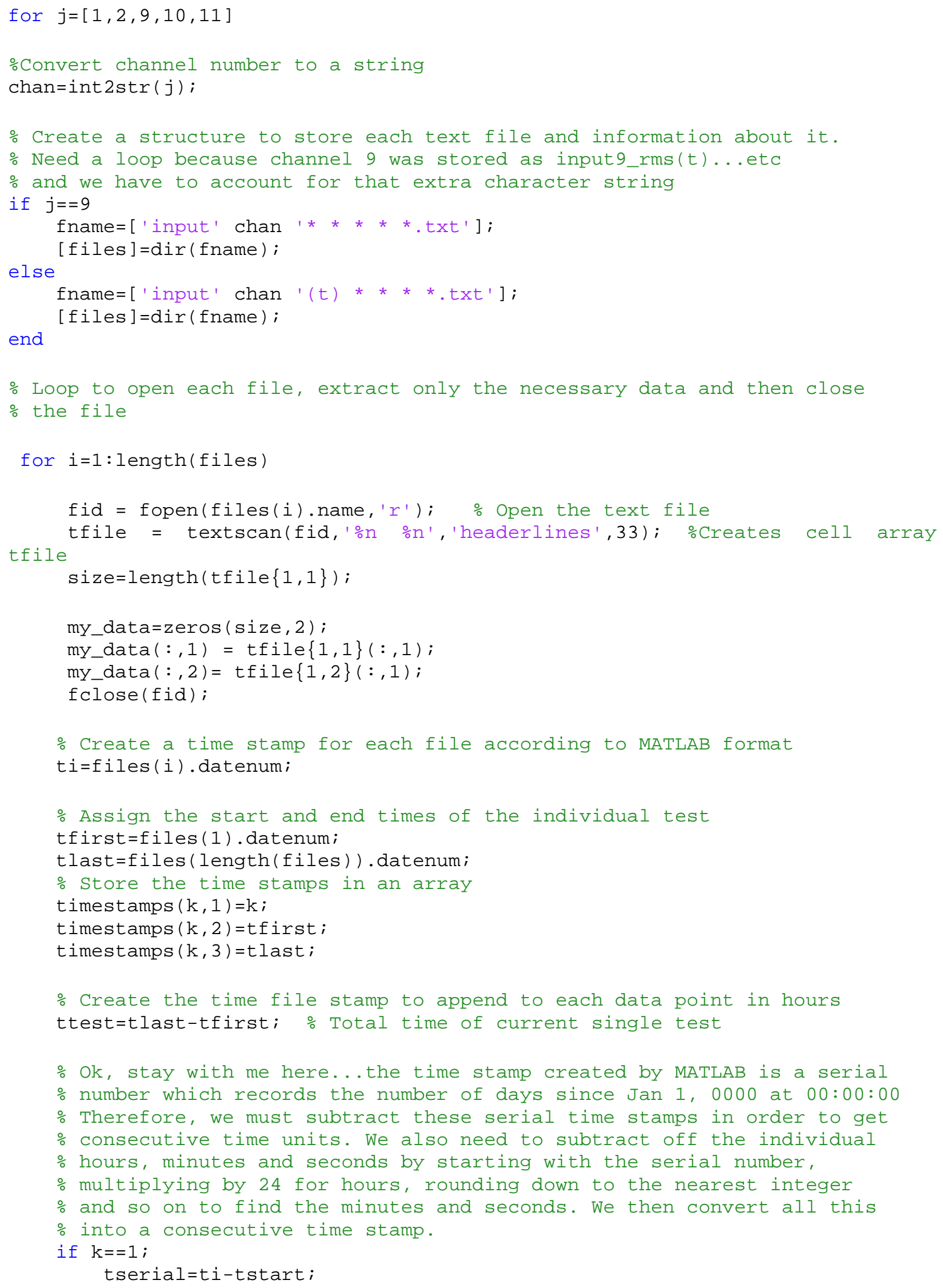




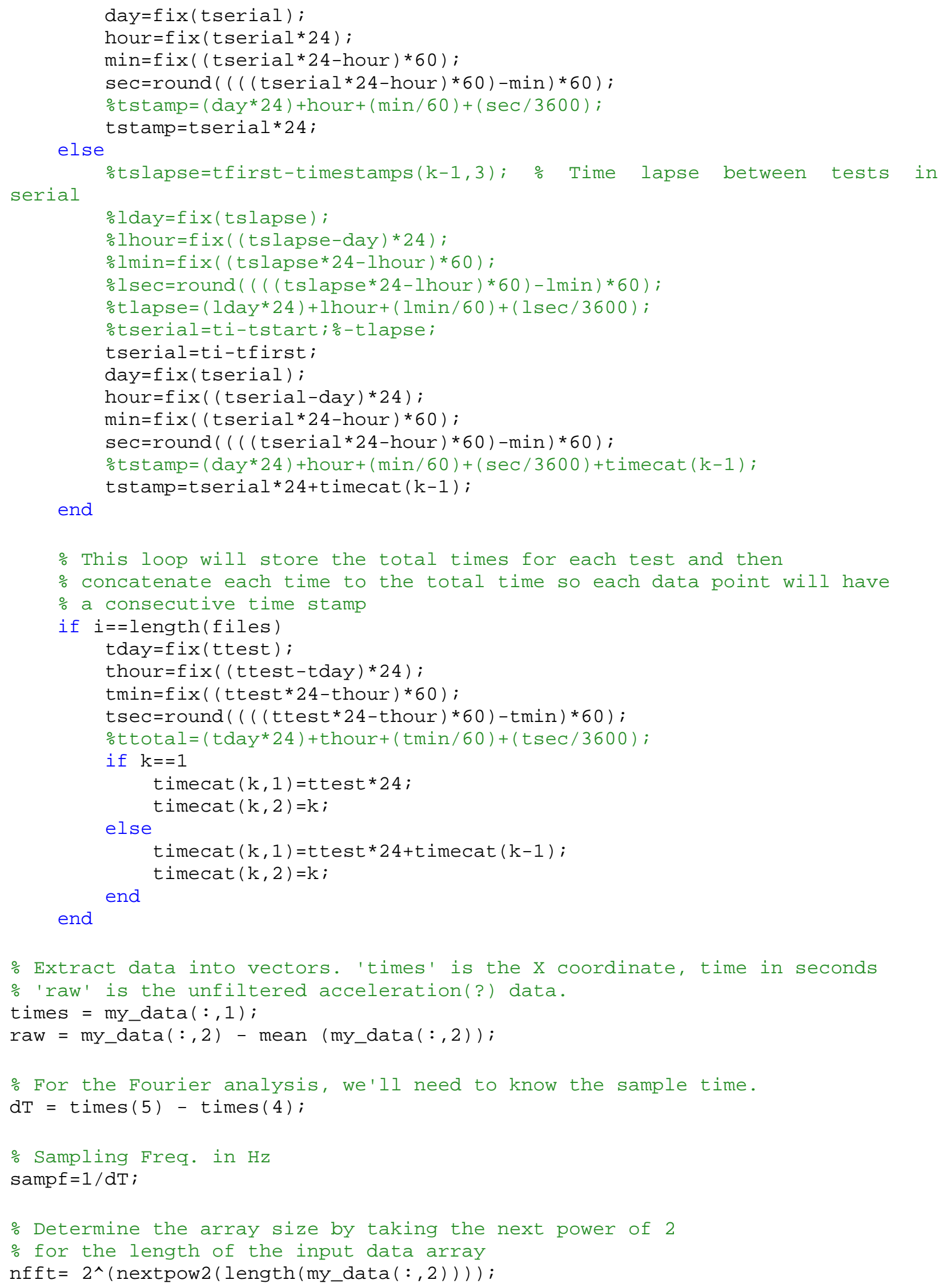




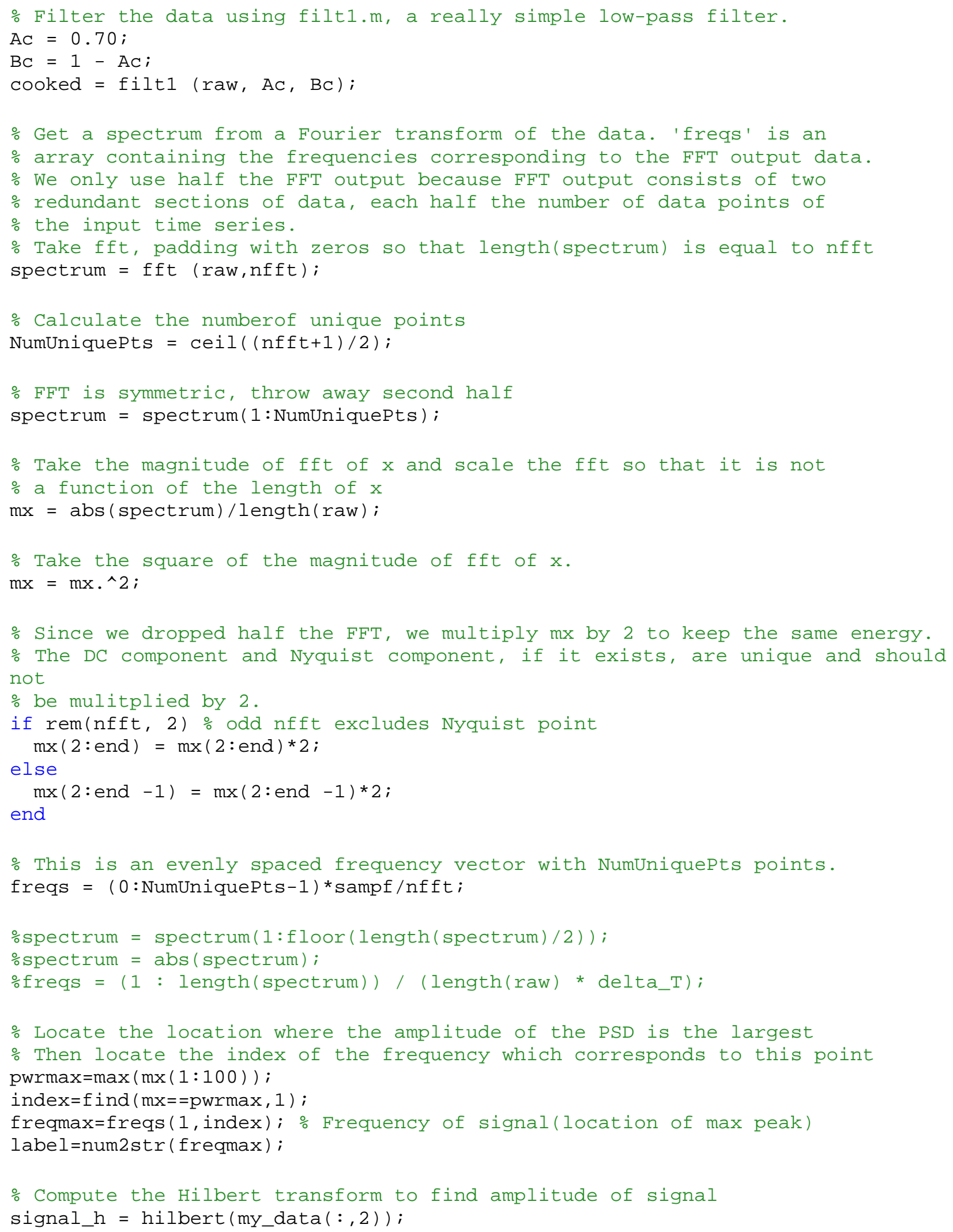




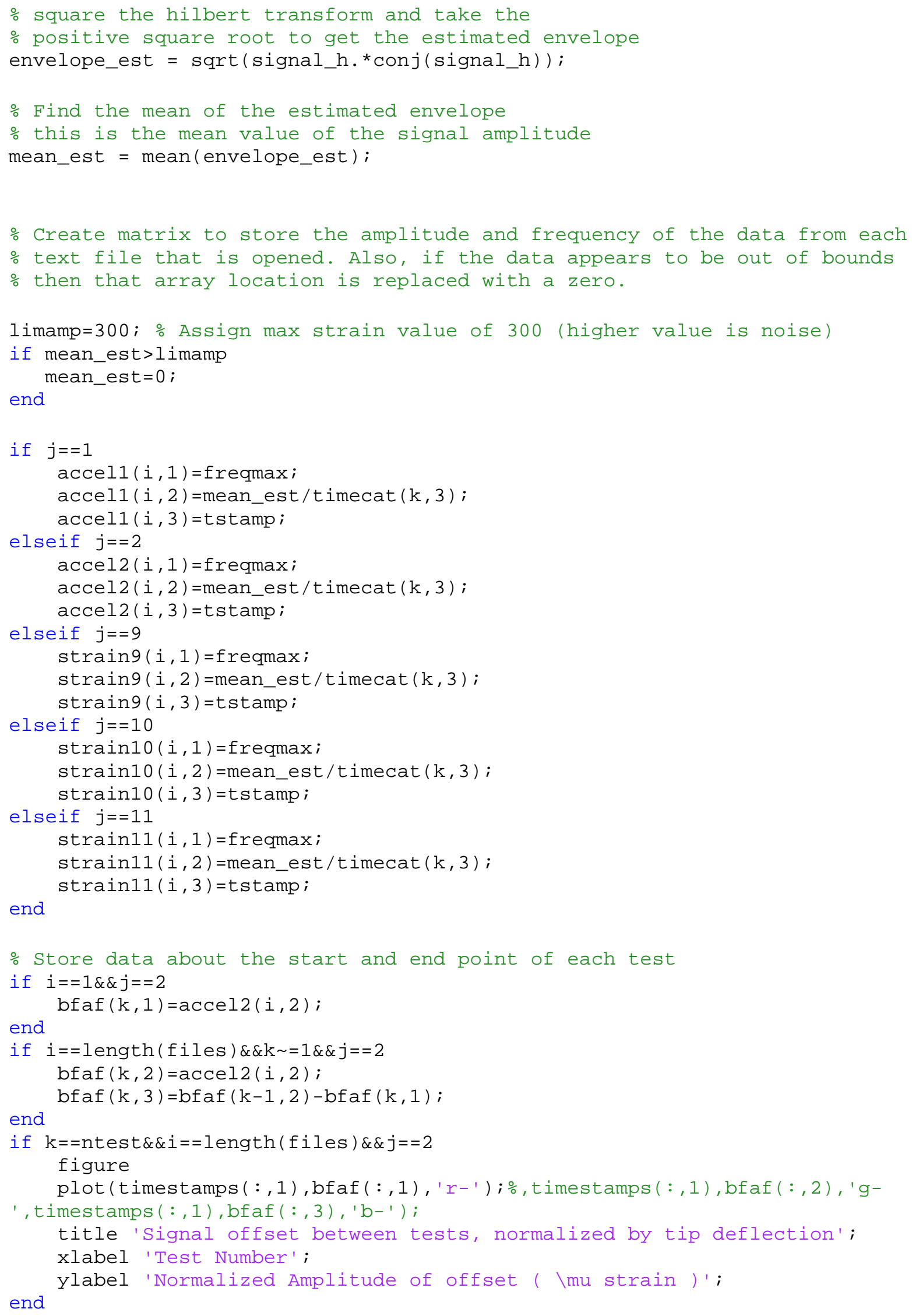




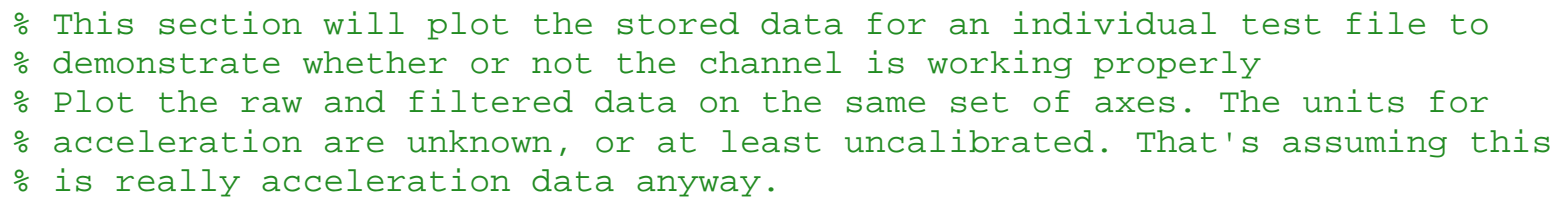




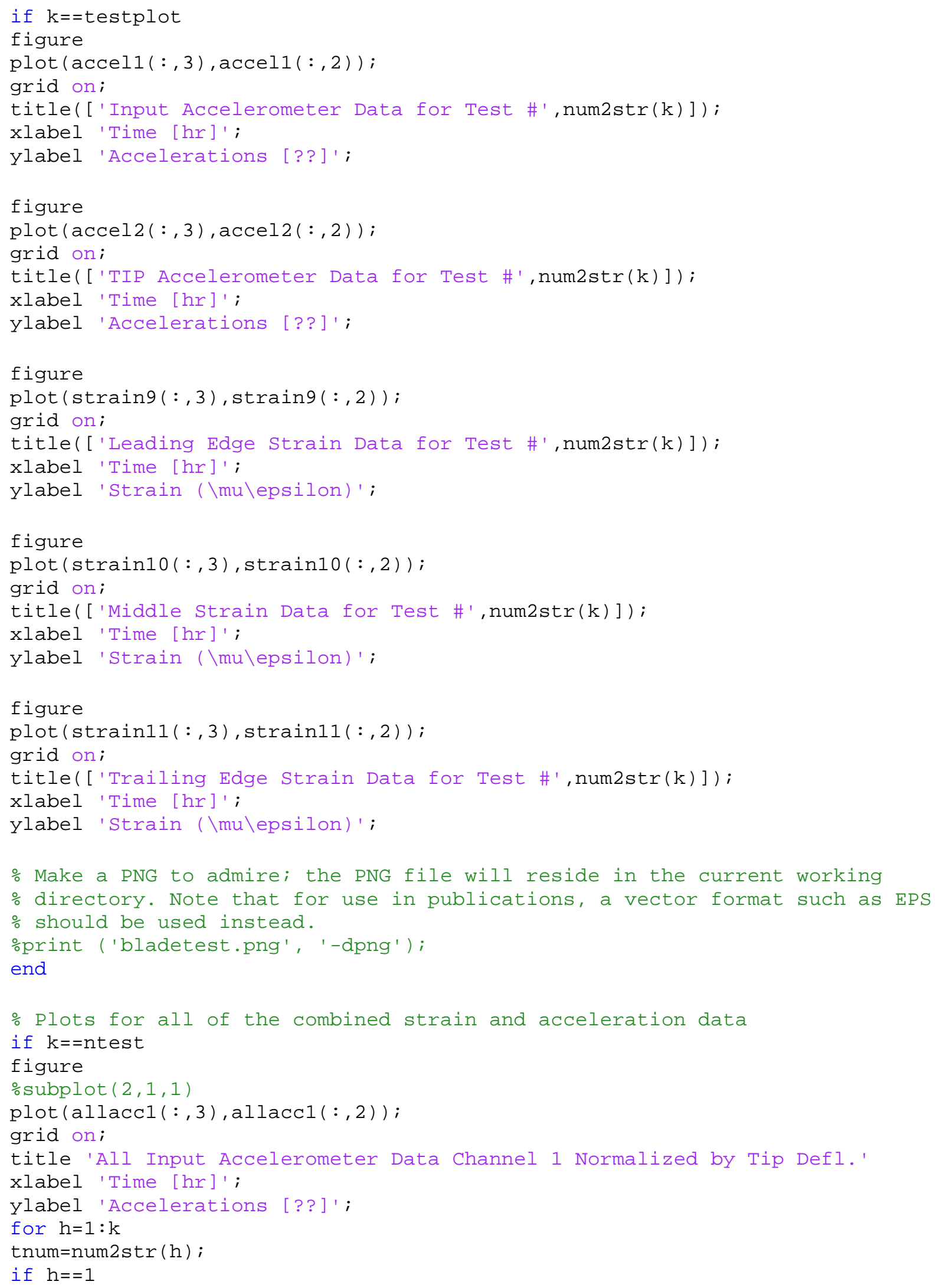




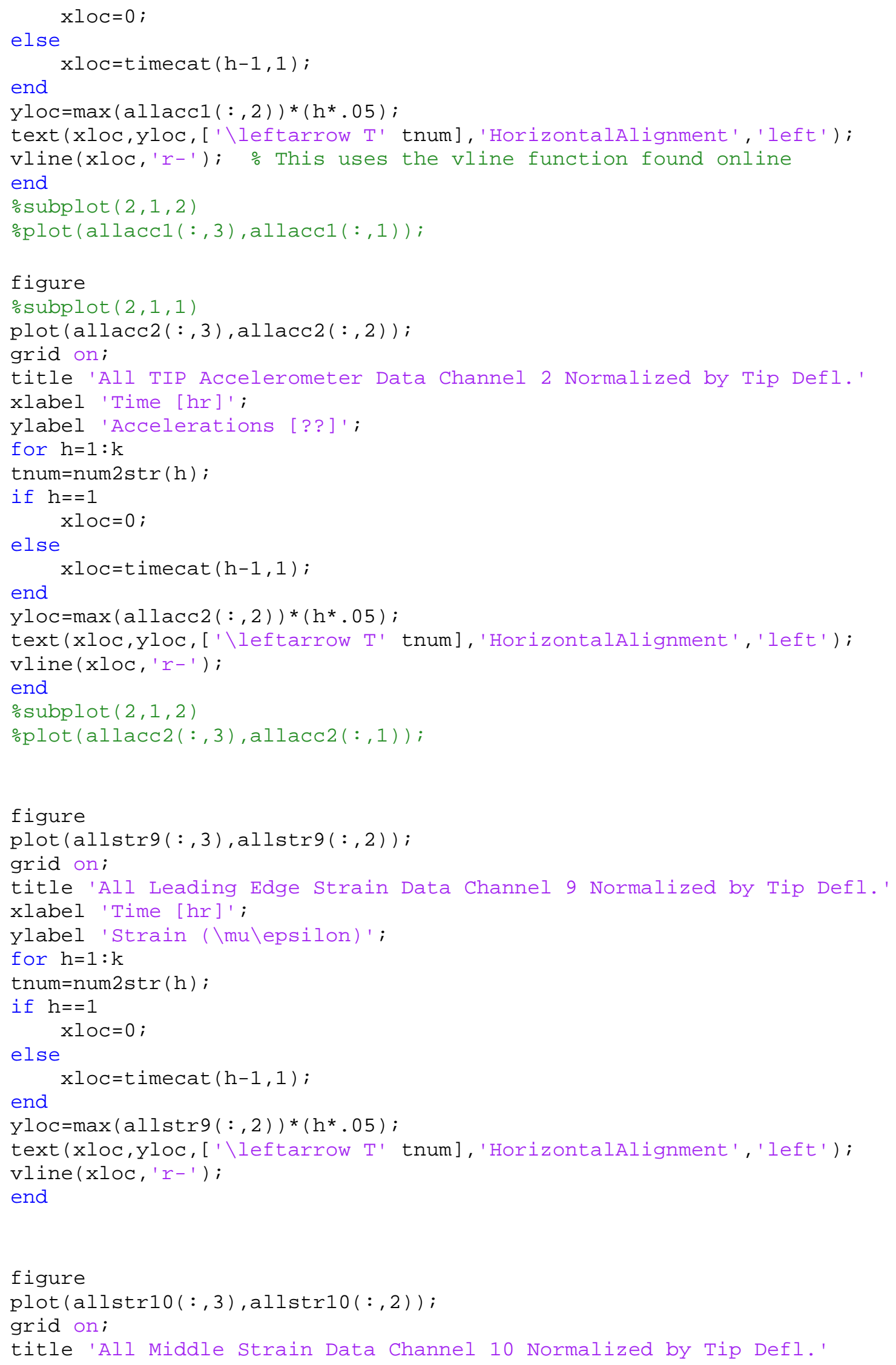




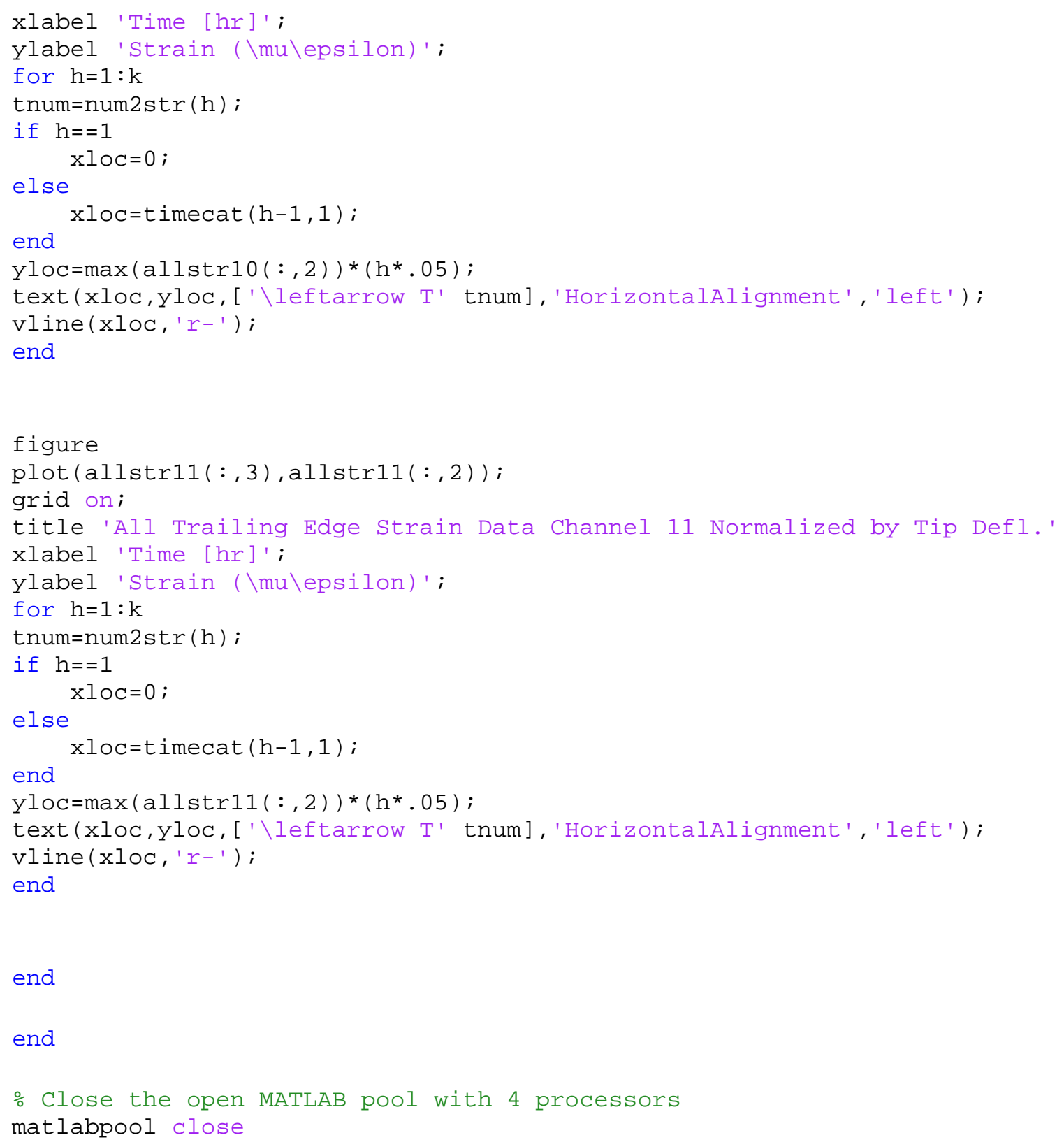




\section{REFERENCES}

Anderson, M. B., J. Noakes, and J. T. D. Slater. "The free Yaw Behavior of Upwind HAWT's." Proceedings of the 15th British Wind Energy Conference. York: K.F. Pitcher, 1993.

Ansell, K. T., and M. P. Tsai. "Principles of Fatigue Loading." (Science Journal) 25, no. 865 (1990).

ASTM D-143. Standard Test Methods for Small Clear Specimines of Timber. American Society of Testing and Materials, 2002.

ASTM E-1049. Standard Practices for Cycle Counting in Fatigue Analysis. American Society of Testing and Materials, 2005.

AWEA. Wind Power Outlook 2008. American Wind Energy Association, 2008.

Bechley, M.E., and P.D. Clausen. "The Dynamic Performance of a Composite Blade from a 5kW Wind Turbine Part II: Predicted Blade Response." (Wind Engineering) 26, no. 5 (2002).

Bechly, M. E. "Aspects of the Dynamics of Small Horizontal Axis Wind Turbines." Department of Mechanical Engineering, The University of Newcastle, 2000.

Betz, A. Windenergie und Ihre Ausnutzzung durch Windmullen. Gottingen, Germany: Vandenhoeck and Ruprecht, 1926.

Bodig, J., and B. A. Jayne. Mechanics of Wood and Wood Composites. Vols. pp 307-312. New York: Van Nostrand-Reinhold, 1982.

Bond, I. P., and M. P. Ansell. "Fatigue Properties of Jointed Wood Composites." (Journal of Material Science) 33 (1998).

Bonfield, P.W., and M.P. Ansell. Fatigue testing of wood composites for aerogenerator rotor blades. Bath, UK: School of Material Sciences, University of Bath, 1993. 
Dally, J. W., and W. F. Riley. Experimental Stress Analysis. Knoxville, TX: College House Enterprises, LLC, 2005.

Devore, J., and N. Farnum. Applied Statistics for Engineers and Scientists. Belmont, CA: Thompson Brooks/Cole, 2005.

Downing, S. D., and D. F. Socie. "Simple Rainflow Counting Algorithms." (International Journal of Fatigue) January p. 31 (1982).

Eggleston, D. M., and F. S. Stoddard. Wind Turbine Engineering Design. New York: Van Nostrand Reinhold Company, 1987.

Epaarachchi, Jayantha A., and Phillip D. Clausen. "The development of a fatigue loading spectrum for small wind turbine blades." Journal of Wind Engineering and Industrial Aerodynamics, 2006: 207-223.

Fitzwater, L.M., and S.R. Winterstein. "Predicting Design Wind Turbine Loads From Limited Data; Comparing Random Process and Random Peak Models." (AIAA) 46 (2001).

Forest Products Laboratory. Wood Handbook: Wood as an Engineering Material. Madison, WI: U.S. Department of Agriculture, 1999.

Glauert, H. Airplane Propellers. Edited by W.F. Durand. Vol. Chapter XI. Berlin: Springer Verlag, 1935.

Grant, A. D., and J. A. Morgan. "Dynamic Loading on an Upwind HAWT in Yawed Flow Conditions: Deterministic Loads." (Wind Engineering) 20, no. 6 (1996): 441-453.

Hansen, and O. L. Martin. Aerodynamics of Wind Turbines. London: Earthscan, 2008.

Have, A.A. WISPER/WISPERX: a sumary paper describing their backgrounds, derivation and statistics. ASME - Wind Energy SED, 1993.

Herbert, J. S. "Fatigue case study and loadinf spectra for wind turbines." IEA Fatigue Expert Meeting. 1994. 
IEC1400-2. Wind Turbines - Part 2: Design requirements for small wind turbines. Geneva, Switzerland: International Electrotechnical Comission, 2006, 99-102.

Jamieson, P. The Design of Wood Epoxy Wind Turbine Blades. Glasgow, Scotland: Howden Wind Turbines, 1990.

Juvinall, R.C., and K.M. Marshek. Fundamentals of Machine Component Design. Hoboken, NJ: John Wiley \& Sons, 2000.

Kelley, N.D., and H.E. McKenna. "The Evaluation of a Turbulent Loads Characterisation System." Proceedings of 17th ASME Wind Energy Symposium. Houston, Texas, 1996.

Kyanka, G. H. Fatigue Cases Incolving the Use of Wood and a Wood Composite. Philadelphia, PA: ASTM, 1994.

Lewis, W.C. "Design Considerations for Fatigue in Timber Structures." (Journal of Structural Division) May, no. pp15-23 (1960).

Manwell, J., J. McGowan, and A. Rogers. Wind Energy Explained. West Sussex: John Wiley \& Sons Ltd., 2002.

Silverstein, Abe. Scale Effect in Clark Y Airfoil Characteristics from N.A.C.A. Full Scale Wind Tunnel Tests. Langley Field: N.A.C.A., 1934.

Thomson, W.T. Theory of Vibrations with Applications. Englewood Cliffs, NJ: Prentice-Hall, 1981.

Van Hule, F.J.L. Validation of Design Criteria for Small Wind Turbines. Gotenburg, Sweden: ECN-RX-96-031, 1996. 